\title{
Imaging Light with Photoelectrons on the Nano-Femto Scale
}

\author{
by \\ Yanan Dai \\ B.S. School of Space Science and Physics, Shandong University, 2013
}

Submitted to the Graduate Faculty of

The Dietrich School of Arts and Sciences in partial fulfillment

of the requirements for the degree of

Doctor of Philosophy

University of Pittsburgh

2019 


\section{UNIVERSITY OF PITTSBURGH \\ DIETRICH SCHOOL OF ARTS AND SCIENCES}

This dissertation was presented

by

\section{Yanan Dai}

It was defended on

April 29, 2019

and approved by

Hrvoje Petek, Professor, Department of Physics and Astronomy

Tao Han, Professor, Department of Physics and Astronomy

David Snoke, Professor, Department of Physics and Astronomy

Daniel Boyanovsky, Professor, Department of Physics and Astronomy

Hong Koo Kim, Professor, Department of Electrical and Computer Engineering

Thesis Advisor: Hrvoje Petek, Professor, Department of Physics and Astronomy 
Copyright @ by Yanan Dai 2019 


\title{
Imaging Light with Photoelectrons on the Nano-Femto Scale
}

\author{
Yanan Dai, $\mathrm{PhD}$ \\ University of Pittsburgh, 2019
}

The interaction of light with solid state quasiparticles, such as excitons and plasmons, on the nanometer-femtosecond spatio-temporal scale illuminates ultrafast physical and chemical processes on surfaces. In this thesis, I report on the generation, control, and spatio-temporal evolution of $2 \mathrm{D}$ evanescent electromagnetic waves confined at the silver $(\mathrm{Ag}) / \mathrm{vacuum}$ interface; such fields, known as surface plasmon polaritons (SPPs), have joint particle-wave nature. SPPs are generated by the interaction of light with the collective response of conduction band freeelectrons of metals. I image and study the ultrafast dynamics of SPP fields by interferometric timeresolved multi-photon photoemission electron microscopy (ITR-mP-PEEM). First, I report on the generation and propagation of SPPs excited on epitaxially grown Ag nanocrystals. The PEEM images record an interference pattern between SPPs and vacuum light, defined by a mismatch in their propagation wave vectors. Next, I explore the light polarization as a control parameter for the SPP generation, where the in-plane and out-of-plane components of optical electric fields couple differently. For equilateral triangle Ag island samples, the SPP interference patterns strongly depend on both the linear and circular polarizations. For circularly polarized light, the SPP coupling depends on the matching between spin angular momenta (SAM) of light and SPPs. The SAM of evanescent waves like SPPs is transverse and points oppositely when the propagation wave vector is reversed; this is known as the photonic quantum spin Hall effect (QSHE). I demonstrate that QSHE affects the function of an SPP lens coupling structure through a vectorial superposition of longitudinally and transversely coupled SPP waves that are launched by TE waves 
(s-polarized) and TM waves ( $p$-polarized), respectively. Finally, I combine my understanding of SPP generation and imaging in a normal-incidence PEEM measurement to explore SPP dynamics when formed by an Archimedean spiral coupling structure. The geometrically defined phase structure of such SPP fields generates plasmonic vortices, whose singularities and time evolution are imaged by PEEM. Based on simulations, I conclude that the SPPs SAM distribution at the vortex core has a stable topological texture of a Néel type Skyrmion, and experimentally locate it by imaging the SPP field singularities. 
1.0 Introduction ................................................................................................................................................ 1

1.1 Collective Electron Oscillations..................................................................................... 2

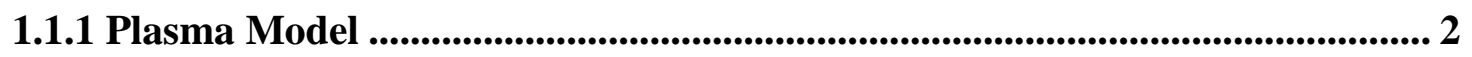

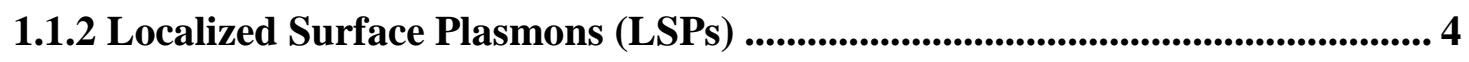

1.1.3 Surface Plasmon Polaritons (SPPs) ......................................................... 7

1.2 Techniques of Imaging Plasmon Related Phenomena ................................................ 12

1.2.1 Photoemission Electron Microscopy (PEEM) ............................................ 13

1.2.2 Low Energy Electron Microscopy (LEEM) .................................................... 17

1.2.3 Low Energy Electron Diffraction (LEED)....................................................... 19

2.0 Experimental Methods ................................................................................................................... 21

2.1 UHV Components of LEEM/PEEM................................................................... 21

2.1.1 Cold Field Electron Emission Gun ..................................................... 21

2.1.2 Electron Optics ................................................................................................ 22

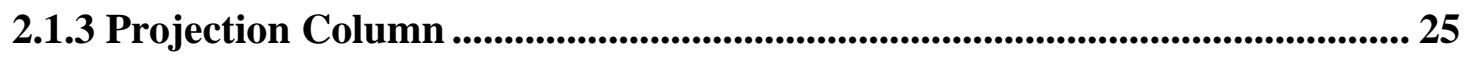

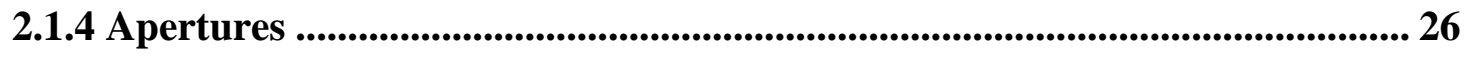

2.2 Sample Preparation .................................................................................................................... 27

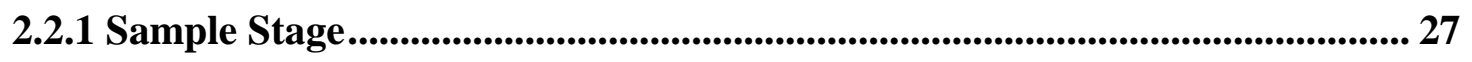

2.2.2 Silicon Substrate Preparation .............................................................................. 28

2.2.3 Single Crystal Silver Pyramids and Wires ..................................................... 29

2.2.4 Single Crystal Silver Islands ............................................................................ 30

2.2.5 Polycrystal Silver Film........................................................................... 31 
2.2.6 Surface Structuring by Focused Ion Beam ......................................................... 32

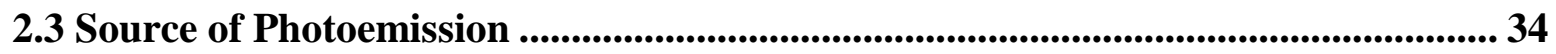

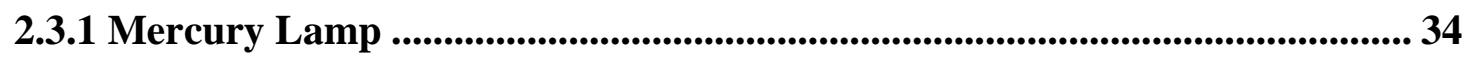

2.3.2 Non-colinear Optical Parametric Amplifier (NOPA) .................................. 34

2.3.3 Interferometric Pump Probe setup.................................................................... 36

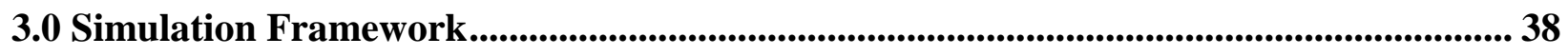

3.1 Finite Difference Time Domain Algorithm ....................................................... 38

3.2 Reproduction of Static PEEM Images ............................................................... 42

3.3 Reproduction of Time-Resolved PEEM ........................................................... 45

3.4 Huygens Principle Algorithm ............................................................................... 48

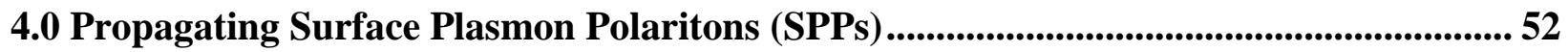

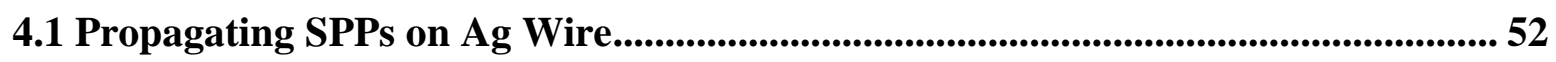

4.1.1 Co-propagation between Light and SPPs ...................................................... 52

4.1.2 Orthogonal Propagation between Light and SPPs ........................................ 56

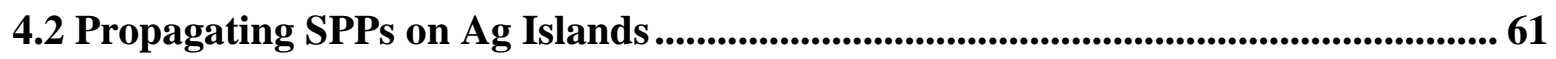

4.2.1 The Vectorial Nature of SPPs Coupling ........................................................ 61

4.2.2 Dynamics of the Propagating SPPs ...................................................................... 65

5.0 Spin Angular Momenta and Chirality of SPPs ............................................................. 68

5.1 Spin-Angular Momentum (SAM) coupled SPPs on Ag Island ................................ 68

5.1.1 Polarization Dependent SPP Excitation and PEEM Imaging ..................... 68

5.1.2 Polarization Dependent Photoemission Yield............................................. 76

5.1.3 Polarization Dependent SPP Phases.......................................................... 83

5.1.4 Longitudinal and Transverse Coupling of SPPs ...................................... 85 
5.2 Dynamics of Spin-Angular Momentum coupled SPPs.................................................. 88

5.3 Quantum Spin Hall Effect on Plasmonic Focusing ................................................. 92

5.3.1 Symmetrical Focus of Plasmonic Lens ...................................................... 92

5.3.2 SAM Dependent Focusing .......................................................................................... 95

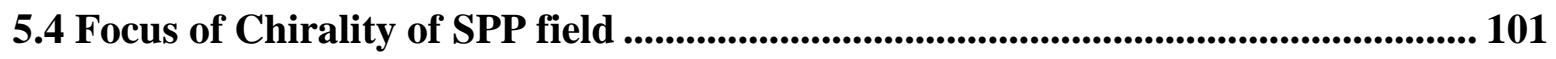

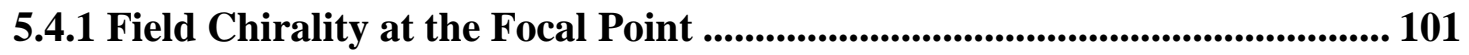

5.4.2 Chirality Density at the Focal Point ................................................................... 104

6.0 Plasmon Orbital Angular Momentum Generation............................................................. 108

6.1 SPP Vortex Generation by Archimedean Spirals............................................... 109

6.1.1 Sample Design and Preparation ..................................................................... 109

6.1.2 Vortex Generation with Circularly Polarized Light ................................... 113

6.1.3 In-plane Component of SPPs Vortex.............................................................. 114

6.2 SPPs Vortex with Pure Geometric Charge ............................................................. 116

6.2.1 Field Evolution of SPPs Vortex Excited by Linearly Polarized Light ....... 116

6.2.2 Static PEEM imaging of SPPs Vortex ................................................... 117

6.2.3 ITR-PEEM imaging of SPPs Vortex Dynamics .......................................... 119

6.3 The Spin Texture of the Plasmonic Vortex .................................................................... 120

6.3.1 Plasmonic Spin Texture ................................................................................ 120

6.3.2 Optical Spin Skyrmion at an SPPs Vortex Core ........................................... 125

6.3.3 Locating Optical Spin Skyrmion ....................................................................... 127

7.0 Summary and Perspectives ................................................................................................ 131

Appendix A Time-Resolved PEEM Software................................................................ 133

Appendix B Huygens Principle Simulation Package .................................................................... 138 


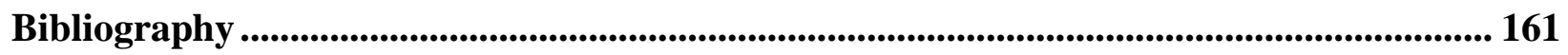




\section{List of Figures}

Figure 1. 1 Schematic of bulk plasmon charge oscillation. ................................................... 4

Figure 1. 2 Schematic of a localized surface plasmon in a spherical particle. ........................... 5

Figure 1. 3 Schematic of the surface plasmon polaritons at metal/vacuum interface.................. 7

Figure 1. 4 Dispersion relation of SPPs at Ag surfaces. .................................................. 10

Figure 1. 5 (a) Schematic of oblique incident PEEM setup. (b) two-photon photoemission PEEM

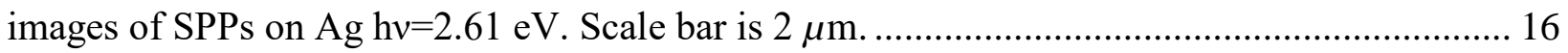

Figure 1. 6 LEEM image of Si(111) surface at sequential Ag coverage. ................................ 19

Figure 1. 7 (a) 1-D chain schematic of electron scattering (b) 2-D LEED pattern of clean $\mathrm{Si}(001)$

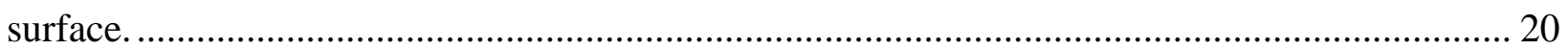

Figure 2. 1 Schematic of the cold field emission gun of FE-PEEM P90 system. ..................... 22

Figure 2. 2 Schematic of the major components of the electron optics in FE-PEEM P90 system. The red and blue paths indicate axial ray and field ray, respectiely......................................... 24

Figure 2. 3 Schematic of the sample stage in FE-PEEM P90 system..................................... 27

Figure 2. 4 (a) Dark field LEEM image of Si(001). (b) Bright field LEEM image of Si(111)... 29

Figure 2. 5 LEEM imge of a Ag wire on $\mathrm{Si}(001)$ substrate ............................................... 30

Figure 2. 6 (a) LEEM image of a $\operatorname{Ag}(111)$ island on $\operatorname{Si}(111)$ substrate. (b) a corresponding LEED

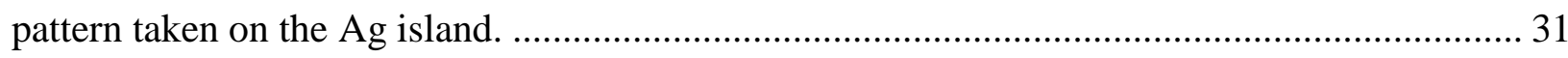

Figure 2. 7 SEM image of polycrystal Ag film with a two slit structures. .............................. 32

Figure 2. 8 SEM images selected plasmonic coupling structures. (a) nanoscale bowtie structure.

(b) chiral plasmonic structure with 6-fold symmetry and varying height. (c) plasmonic lens 
coupling structure. (d) Archimedean spirals plasmonic vortex generator for topological charge of $\mathrm{m}=8$. 33

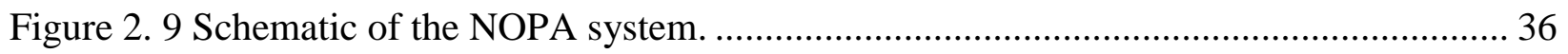

Figure 2. 10 Schematic of Mach Zehnder interferometer....................................................... 37

Figure 3. 1 A unit cell of the Yee grid (inner cube) of in a FDTD algorithm. Within one grid, three electric field components and three magnetic field components are marked by arrows. These components are placed at different spatial locations in the cube to update surrounding fields by the curl equations. 41

Figure 3. 2 Schematic of the 1-D excitation of SPPs at Ag/vacuum interface, and snapshots of simulated $\boldsymbol{E} \boldsymbol{z}$ field along the Ag/vacuum interface. The SPP wave is launched at $-12 \mu \mathrm{m}$, and propagates towards $+x$ direction as the simulation time evolves. Clear interference with a $\lambda_{\mathrm{B}}$ envelope pattern is seen when the incident pulse is close to, but past its maximum. After the pulse has passed, the pure SPP field propagates and decays. 43

Figure 3. 3 Simulated interference pattern based on the fields in Figure 3.2.2, showing distinct periodic fringes that arise from SPP field beating with the $\boldsymbol{E} \boldsymbol{z}$ component of the excitation field with periodicity $\lambda_{\mathrm{B}}$

Figure 3. 4 Snapdhots of time-resolved beating patterns, based on the fields in Figure 3.3. At $0 \mathrm{fs}$, only 4 distinct beats are observed, but at longer $\Delta \boldsymbol{t}$, more beats, due to the $\Delta \boldsymbol{t}$ dependent pumpprobe interaction, appear. 46

Figure 3. 5 2D interferogram of the SPP field discussed in this section. The abscissa represents the pump-probe delay, and the ordinate the propagation distance. The color scale designates the spatially varying beating amplitude. The dashed line marks the space-time propagation of SPPs beating pattern with the slope giving the SPP phase velocity. 47 
Figure 3. 6 Schematic of the vectorial coupling on a simple slit structure

Figure 3. 7 (a) Simulated SPP phase upon changing the unit vector continuously with LCP excitation. (b) Photoemission yield from a single point source as a function of quarter wave plate angle, which defines the light elipticity. 51

Figure 4. 1 (a) Experimental 1PPE-PEEM image of the lateral dimensions a $20 \mu \mathrm{m}$ long Ag wire acquired with an $\mathrm{Hg}$ lamp $(\mathrm{h} v=4.89 \mathrm{eV}) .(\mathrm{b}-\mathrm{f})$ Wavelength-dependent 2PP-PEEM measurements of SPPs on the same wire as in (a). The red arrow indicates the propagation direction of the excitation light. Light entering at $70^{\circ}$ from the surface normal from the left, dominantly excites SPPs from the left edge. The excitation photon energies are indicated in $(b-e)$. 53

Figure 4. 2 Calculated and measured (dots) beating period $\boldsymbol{\lambda} \boldsymbol{B}$ (color scale) for the incident excitation at $\theta=70^{\circ}$ as a function of excitation wavelength and the angle $\gamma$ between the propagation k-vectors of the in-plane component of the external and the SPP fields. The schematic on the right shows the vectors of an example coupling angle where $\gamma$ is nonzero. 55 Figure 4. 3 PEEM image of a $2.5 \mu \mathrm{m}$ Ag wire excited by $3.3 \mathrm{eV}$-polarized light, when the kvector of light orthogonal to the wire long axis. Scale bar is $500 \mathrm{~nm}$. 57 Figure 4. 4 (a) schematic of the simulation setup for wire orthogonal to the k-vector of the excitation light. (b) Time-averaged field distribution at the cross section marked in (a)........... 58 Figure 4. 5 SPPs dispersion relation of the orthogonally placed Ag wire .............................. 59 Figure 4. $6 \mathrm{Ag} / \mathrm{Si}$ interface plasmon modes at selected photon energies 60 Figure 4. 7 Experimental PEEM image of the SPPs on a truncated triangular Ag(111) island, excited with $p$-polarized $h v=3.21 \mathrm{eV}$ light. Red arrow indicates the direction of the k-vector of the incident light. White dashed lines demark the island edges. 
Figure 4. 8 Simulated PEEM image of SPPs excited on ain a truncated triangular $\operatorname{Ag}(111)$ island, excited with p-polarized $h v=3.21 \mathrm{eV}$ light. $\boldsymbol{k i n}$ is the k-vector of incident light, $\boldsymbol{k s p p}$ is the $k$ vector of the SPP field and $\boldsymbol{k} \boldsymbol{B}$ is the k-vector of the beating field. $\varphi$ indicates the phase of external light at edges that can couple forward propagating SPP waves, $\delta$ indicates the phase of external light at edges that can couple back-propagating SPP waves. (1)-(4) indicate the the island edges that couple to SPP field in the sequence of excitation time. $\boldsymbol{E} \|$ indicates the in-plane polarization of the external light. 64

Figure 4. 9 ITR-PEEM images from a truncated triangle $\operatorname{Ag}(111)$ island obtained by double-pulse pump-probe excitation at $\mathrm{h} v=2.7 \mathrm{eV}$ with delays between the pulses of $\Delta \mathrm{t}=0$ (a) and $\Delta \mathrm{t}=18.3$ fs (b). The intensity of (b) is multiplied by a factor of 10 for better visibility. (c) 3D intensity plot integrated in the horizontal direction over part of the island that is indicated by the dashed rectangle in (b) in a selected delay time window spanning $6.4 \mathrm{fs}$. Clear progression of the beating maxima reveals the motion of SPP waves. The slope of the beating evolution marked by the dashed arrow gives the SPP phase velocity. 66

Figure 5. 1 1P-PEEM image of the $\operatorname{Ag}(111)$ surface of a single crystal island. The Greek letters label the edges of the island.

Figure 5. 2 Annotations of vectors and related physical quantities. $\boldsymbol{n} \perp$ and $\boldsymbol{n} \|$ are unit vectors normal and parallel to sample surfaces. $\boldsymbol{k s p p}$ is the k-vector of the SPP wave, $\boldsymbol{k} \boldsymbol{L}$ is the in-plane $k$-vector of the excitation light. $\boldsymbol{S} \perp \boldsymbol{s p p}$ is the transverse SAM of the SPP fields, and $\boldsymbol{S} \| \boldsymbol{L}$ is the in-plane SAM of the external light field. $\boldsymbol{E} \|$ and $\boldsymbol{E} \perp$ are the in- and out-of-plane components of the external field, respectively. 70

Figure 5. 3 PEEM image of the truncated Ag island excited by $\mathrm{h} v=2.70 \mathrm{eV}$, for $p$ - and $s$-polarized light excitations. 71 
Figure 5. 4 PEEM image of the truncated Ag island at $460 \mathrm{~nm}$, for selected linearly polarized light excitations.

Figure 5. 5 PEEM image of the truncated Ag island at $460 \mathrm{~nm}$, for left and right circularly polarized light excitations. 74

Figure 5. 6 Simulated 2PP PEEM images of the Ag island at $460 \mathrm{~nm}$ excitation under linear (a-d) and circular (e-f) excitations, showing the asymmetric interference patterns. The color scale is normalized to (a). 75

Figure 5.7 (a)(b) Simulated 2P-PEEM images based on Huygens-Fresnel model, for left and right polarized light excitations, respectively. 76

Figure 5. 8 Circular plots of the experimental (makers) and FDTD simulated (lines) 2PP yields from the regions indicated in Figure 5.3(a) as a function of $\xi$. The radial axis length represents normalized PEEM yield, while the polar axis is the retardation plate angle $\xi$, where one cycle corresponds to $\pi$ rotation, for tuning of the linear and circular polarizations. The effective polarizations are also indicated for selected $\xi$. (a)(b) Normalized 2PP intensities from the coupling at the $\boldsymbol{\alpha}$ edge (the central rectangle of Figure 5.3(a)) for the linearly and circularly polarized excitations. (c)(d) Linearly and circularly polarized excitation intensities taken from $\boldsymbol{\beta}$ (blue) and $\boldsymbol{\beta}^{\prime}$ (red) edges (Figure 5.3(a)), showing tilting of the distribution dumbbells by $\sim \pm 11^{\circ}$ and $\sim \pm 5^{\circ}$, with respect to the $\boldsymbol{\alpha}$ edge. The helicity dependent asymmetry is caused by directional coupling to SPPs due to the SAM matching. 78

Figure 5. 9 Experimental data and analytical fitting of the experimental photoemission yields at various regions of interest in comparison with the numerical simulation results in Figure 5.8. (a)(b) show the 2PP yield taken at the central square region in Figure 5.3(a). (b)(d) show the 2PP yield in the squared region close to the $\beta$ edge in Figure 5.3(a). 81 
Figure 5. 10 Plot of the polarization dependent initial phase of SPPs launched from the $\beta$ and $\beta$, edges relative to the p-polarized excitation acquired on left (blue) and right (red) side of the $\mathrm{Ag}$ island. The $\xi$ angle is shown on the top axis, and the corresponding laser polarization at the bottom of each graph. (a) Initial SPPs phase, $\boldsymbol{\Phi s p p}$, for linearly polarized excitation, showing its variation with a period of $\boldsymbol{\xi}=\boldsymbol{\pi} \mathbf{2}$. The phase from $\beta$ edge changes slowly when $-\boldsymbol{\pi} \mathbf{4}<\boldsymbol{\xi}<\mathbf{0}$, more rapidly for $\mathbf{0}<\xi<\boldsymbol{\pi 4}$, and has sudden jumps at $\pm \boldsymbol{\pi 4}$. (b) $\boldsymbol{\Phi} \boldsymbol{s p p}$ with circular excitation a smoother variation for all polarizations occurs. When the in-plane SAM of light $(\boldsymbol{S} \| \boldsymbol{L})$ and the transverse SAM of SPPs $(\boldsymbol{S} \perp \boldsymbol{s p p})$ are parallel $\boldsymbol{\Phi} \boldsymbol{s p p}$ is advanced $(\beta$ edge at $-\boldsymbol{\pi} \boldsymbol{4})$, and when they are anti-parallel it is retarded. 84 Figure 5. 11 (a) The $\mathrm{z}$ component of the simulated total polarization field $\boldsymbol{P z}=\boldsymbol{P t o t x}, \boldsymbol{y}, \boldsymbol{\tau} \cdot \boldsymbol{n} \perp$ taken $6 \mu \mathrm{m}$ away from island symmetry axis [dashed arrow in Fig. 1(d)] at $53.5 \mathrm{fs}$ after the pulse maximum interacted with the coupling edge for various linear (blue) and circular (red) polarization states of light. All of the polarization fields can be obtained by properly superimposing p- and spolarized light based on the Jones transformation matrix for phase retarders. The intermediate polarizations show both amplitude and phase changes in agreement with experiment. (b, c) Profiles of $\boldsymbol{P} \boldsymbol{t}$ and $\boldsymbol{P} \boldsymbol{l}$ components and their superimposed SPP field $\boldsymbol{P}$, based on Huygens pricple simulations, at $53.5 \mathrm{fs}$ acquired from the same positions as in the FDTD simulation. Clear amplitude asymmetry and phase change is observed in $\boldsymbol{P}$ as well as the calculated PEEM profiles, for LCP and RCP excitation. 86

Figure 5. 12(a)(b) Snapshots of experimental ITR-2PEEM images excited by RCP light at pulse delay time $\Delta \boldsymbol{t}=0$ fs and $\Delta \boldsymbol{t}=27.6 \mathrm{fs}$, respectively. (c)(d) The corresponding snapshots of the simulated ITR-2PEEM images for the delays in (a) and (b) based on the Huygens-Fresnel model. At $\Delta t=0 \mathrm{fs}$, distinct beating patterns are only visible near the island coupling edges. At $\Delta t=27.6$ 
fs, the beating patterns away from the edges become more pronounced due to the interference between the probe pulse and the pump excited SPP field. 89

Figure 5.13 (a) Spatial displacement of the second SPPs beating maximum with respect to $\Delta t$ (The traces are displaced by $2 \mu \mathrm{m}$ along the vertical axis to enable visual comparison.). The four datasets correspond to SPPs launched from both the $\beta$ and $\beta^{\prime}$ edges for both the LCP and RCP light. The slopes observed from fitting the spatial displacements, which give the phase velocity of SPPs, are similar because the phase velocity of SPPs is independent of polarization. (b) Normalized I2PC traces taken at various points at the intersections of the dashed and solid arrows in 5.12(b) away from the $\beta^{\prime}$ edge. A two-pulse autocorrelation trace, taken at $\beta^{\prime}$ edge, is also plotted as a reference. A propagating interference envelope is observed in the tail of the I2PC trace when measuring $>10$ $\mu \mathrm{m}$ from the coupling edge. The envelope shift with $\Delta t$ gives the SPP group velocity............... 91 Figure 5. 14 (a) Schematic diagram of ITR-MP-PEEM excitation. $\boldsymbol{E v}$ denotes the vacuum level of Ag. (b) PEEM image excited with an $\mathrm{Hg}$ lamp $(\mathrm{h} v=4.9 \mathrm{eV}$ ) showing geometry of the plasmonic lens. 93

Figure 5. 15 (a)(b) Experimental and (c)(d) calculated time-resolved 2P-PEEM frames of the lens excited by p-polarized pulses at 0 and $29.4 \mathrm{fs}(21 \times 2 \pi$ radians $)$ pump-probe delay times. The signal enhancement away from the lens after the delay in (b) and (d) is caused by SPP focusing as its phase fronts propagate from the coupling edge. 94 Figure 5. 16 Experimental (left) and calculated (right) ITR-MP-PEEM images of the plasmonic lens excitation by (a-d) LCP and (e-h) RCP light. The SPP waves travel and focus asymmetrically at $\sim 12.5 \mu \mathrm{m}$ in front of the lens. The distinctly asymmetric interference patterns for excitation with the opposite ellipticity manifest the spin-Hall effect. 96 
Figure 5.17 (a) The calculated z-component of the total polarization field taken at $\mathrm{x} \sim 6.5 \mu \mathrm{m}$ for $\Delta \mathrm{t}=0$ [the dashed white line in Figure 5.16(b)] for selected polarizations. The period of the fast, weak oscillations is wavelength of the SPP field. For p-polarized light, a symmetric field distribution is excited because it is parallel and symmetric to the optical plane. For s-polarized light, the distribution is anti-symmetric, because its optical field is antisymmetric with respect to the optical plane creating a $\pi$-phase shift for coupling on the opposite sides of the lens structure. The field distribution for circularly polarized light has an asymmetry along y, which can be reconstructed by superimposing the p- and s- distributions with the appropriate amplitudes and phases. The dashed lines mark extrema in $\boldsymbol{P z}$ at $\mathrm{y} \sim \pm 3 \mu \mathrm{m}$, showing the phase differences for all polarizations. (b) IPEEM line profiles integrated in time according to Eq. (1.22) at the same location as in (a). The time averaging washes out the asymmetry for the linearly polarized, but not the CPL light. 100

Figure 5. 18 (a) Schematic of the plasmonic lens based on Huygens principle. SPP waves from three selected origins are considered (right-most and left-most points) propagating with $\boldsymbol{k 1}, \boldsymbol{k} \mathbf{2}$ and $\boldsymbol{k} \mathbf{3}$ vectors over distances labeled as $\boldsymbol{L 1}, \boldsymbol{L} \mathbf{2}$ and $\boldsymbol{L} \mathbf{3}$ respectively. The point of consideration is labeled by P, which is above the focal point F. (b) The simulated $\boldsymbol{E z}$ field at $500 \mathrm{~nm}$ above the focal point, from the waves in (a). Meeting at $\mathrm{P}$, the $\boldsymbol{k} \mathbf{1}$ field precedes the others, causing the total field to circulate, which makes it chiral. (c)-(d) colormaps of the $\boldsymbol{E} \boldsymbol{z}$ component of SPP fields coming to the focus, superimposed with the in-plane polarization states. The polarization states form L-line singularity on the optical axis of SPP wave excited with p-polarized light, but above and below the L-line they circulate counterclockwise, and clockwise. The red polarization ellipse is formed by the field profiles in (b). For LCP and RCP excitation the focus and the field ellipticity shifts up and down 103 
Figure 5. 19 (a)-(c) Calculated normalized chirality density $\mathbf{C n o r m}$ for p- and circularly polarized excitations. The horizontal solid lines mark the lens optical axis $(y=0)$, and the dashed lines mark the maxima of the $\mathbf{C n o r m}$ focus. In the left panel, the total $\mathbf{C n o r m}$ includes the incident field, while the right panel shows chirality density due to SPPs only. (d)(e) are total chirality line profiles along the vertical (d) and horizontal (e) dashed lines in the left panels of (a)-(c). Clear enhancement is observed for CPL excitations. The extremum of the chirality near the focal point (guided by dashed lines) show that the chirality enhancement is only shifted in the y direction dependent on the polarization of light. For p-polarized light, the chirality density inverts in the $y$ direction through the focal spot. For circularly polarized light, chirality is mostly dominated by the chirality of the incident light, with a clear focus following the SPP field. 105 Figure 6. 1 Schematic of an Archimedean spiral of topological charge $m=1$. 110 Figure 6. 2 Schematic of single slit and concentric slit, multiple opening, Archimedean spirals both with a topological charge $m=2$.

Figure 6. 3 SEM imagse of concentric multiple opening Archimedean spirals with topological charges $m=4$ (a) and $m=8$ (b). 112 Figure 6. 4 Simulated evolution of the $z$-component of the SPP fields launched by excitation of an $m=1$ Archimedean spiral by RCP (top) and LCP (bottom) light. When the total OAM of the SPP field is $L=2$, four petals orbit around the vortex core, but when $L=0$, a plasmonic focus and no phase singularity occurs. 113

Figure 6. 5 Coordinate system of the circular plasmonic coupling strucutre. 115 Figure 6. 6 Calculated field distributions of an L=1 plasmonic vortex. (a) $\boldsymbol{E} \boldsymbol{z}$ field, (b) $\boldsymbol{E} \boldsymbol{r}$ field and (c) $\boldsymbol{E} \boldsymbol{t}$ field. The arrows in (a) indicate the Poynting vectors. The image size is $2 \lambda \boldsymbol{s p p}$... 116 
Figure 6. 7 Simulated evolution of the $z$-component of SPP field, launched at an $m=2$ Archimedean spiral by linearly polarized light.

Figure 6. 8 Schematic of the experimental setup for SPP vortex generation. 118

Figure 6. 9 (a) Experimental PEEM image of the SPPs vortex launched from $m=2$ Archimedean spiral with $550 \mathrm{~nm}$ linearly polarized light. (b) Simulated time-averaged $z$-component of the SPP fields. 119

Figure 6. 10 Sequence of experimental time-resolved PEEM images within one cycle of light exitation. 120

Figure 6. $11 \mathrm{~K}$-vector and spin direction of an $L=1$ SPPs vortex. 121

Figure 6. 12 3D spin texture of an $L=1$ SPPs vortex. 123

Figure 6. 13 Skyrmion density map of the $L=1$ vortex. 124

Figure 6. 14 Topological charge dynamics of optical spin Skyrmion. 125

Figure 6. 15 Skyrmion density of the optical SAM asscociated with the SPPs vortex launched by $550 \mathrm{~nm}$, linearly (horizontally) polarized light on Ag surface. 126

Figure 6. 16 L-line map of the $L=1$ SPP vortex excited by RCP light. (b) The L-line map of the $m=2$ SPP vortex cited by the lineary polarized light. 128

Figure 6. 17 Fourier spectrum of the time-dependent PEEM data. The dashed box maks the firstorder oscillitory photoemission component. 129 Figure 6. 18 Fouier filtered PEEM images, with time-delay corresponding to the PEEM images in Figure 6.10. 129 Figure 6. 19 An optical flow image determining the experimental observed L-line map of the $m=2$ SPP vortex excited by linearly polarized light. 130 


\section{List of Equations}

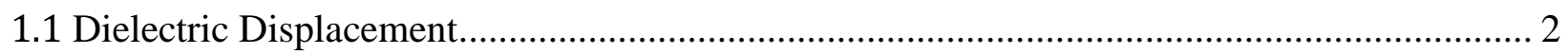

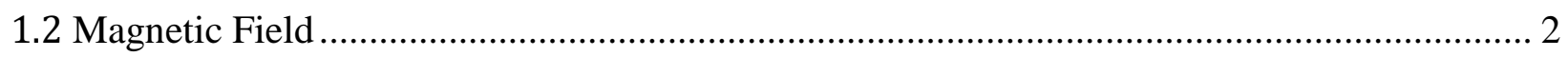

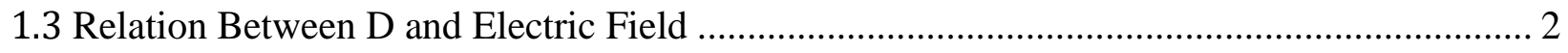

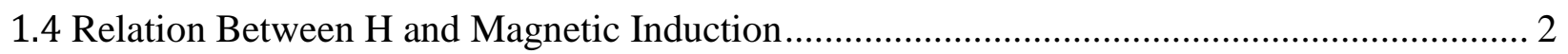

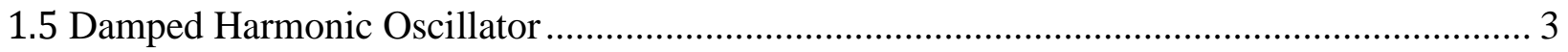

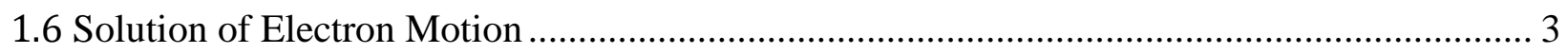

1.7 Specific Form of Dielectric Displacement ………….................................................... 3

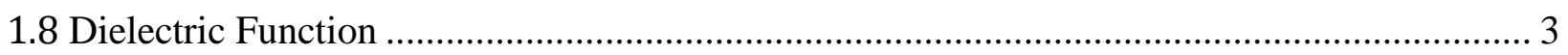

1.9 Electric Potential of Spherical Metal Particle ......................................................................... 5

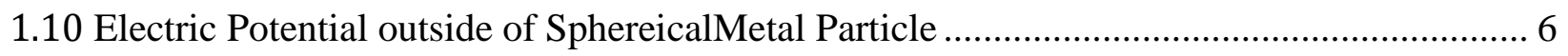

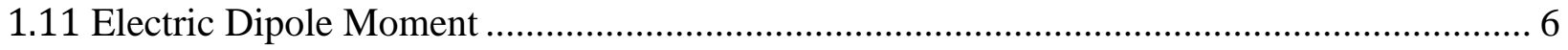

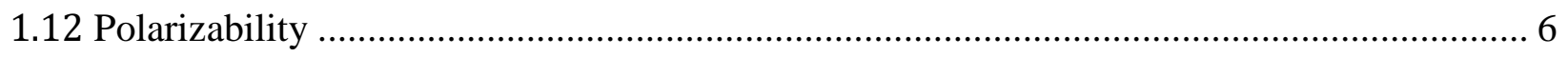

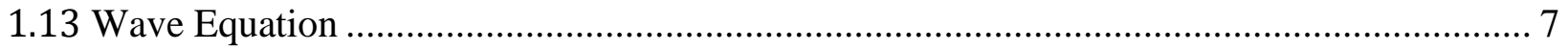

1.14 Solution to Surface Plasmon Polaritons in Vacuum .......................................................... 8

1.15 Solution to Surface Plasmon Polaritons in Metal................................................................. 8

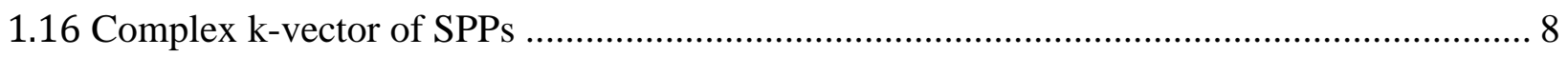

1.17 General Expression for the E field of SPPs..................................................................... 10

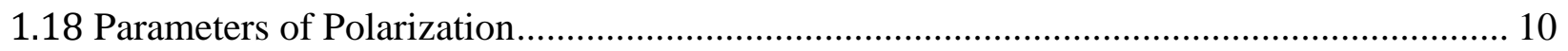

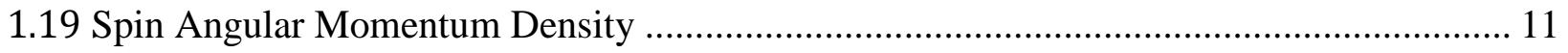

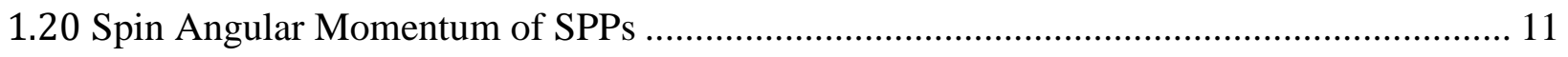


1.21 Details of Spin Angular Momentum Density .................................................................. 11

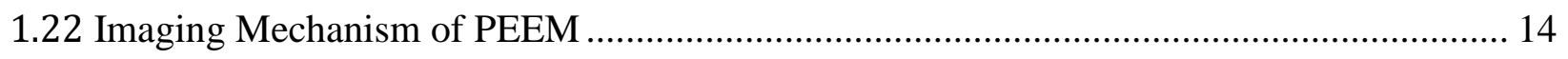

1.23 Surface Plasmon Polaritons Beating Period ...................................................................... 15

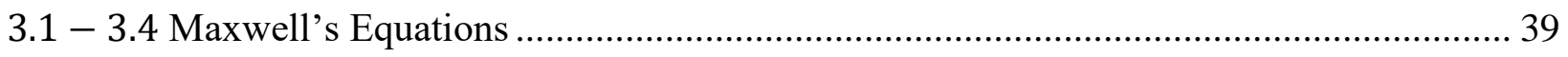

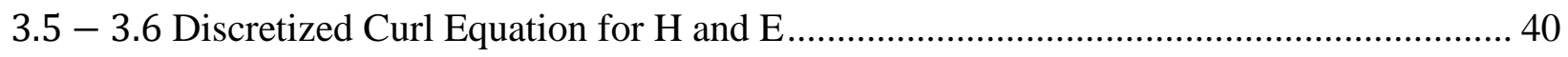

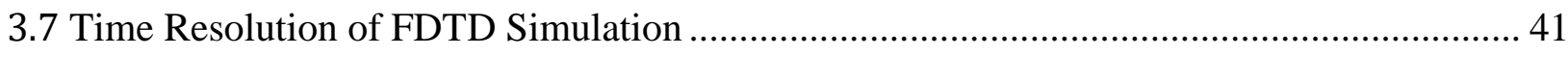

3.8 Calculation of PEEM images based on Time-dependent Fields ........................................... 45

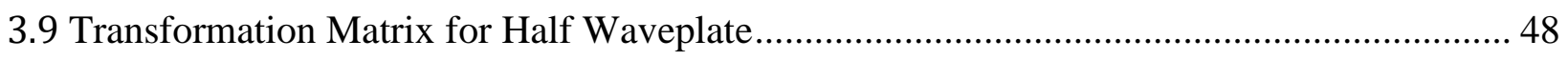

3.10 Transformation Matrix for Quarter Waveplate .................................................................. 48

3.11 Total SPP Fields in Huygens Principle Simulation .............................................................. 48

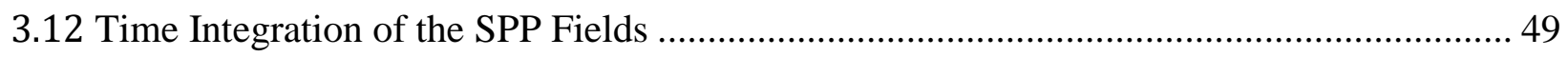

3.13 PEEM Image Caculation in Huygens Principle Simulation................................................. 50

5.1 - 5.3 Cartesian Components of the E Field after Half Waveplate ........................................ 80

5.4 Photoemission Yield as a Function of Linear Polarization ...................................................... 80

5.5 - 5.7 Cartesian Components of the E Field after Quarter Waveplate .................................... 82

5.8 Photoemission Yield as a Function of Circular Polarization............................................... 82

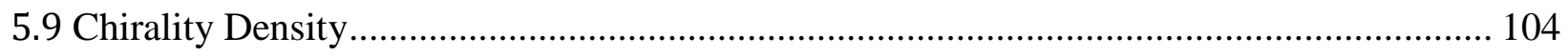

6.1 - 6.3 SPP Vortex Fields in Cylindrical Coordinate......................................................... 115

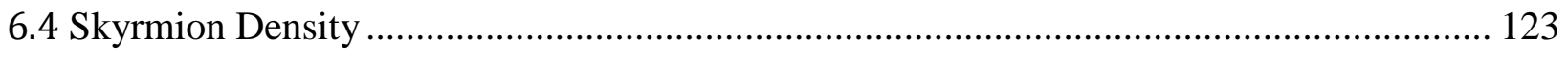




\subsection{Introduction}

Light-matter interactions are of great interest to physicists and chemists. Particularly, light interaction with noble metals is fundamentally important because it is the basis of the first optical device, a metal mirror, and in fundamental research and applications, because it represents the response of a free electron gas in solid state to electromagnetic fields. Among noble metals, silver holds a unique place because its interband absorption starts only in the UV range, giving it a low loss in the visible spectrum. Because of this, the real part of its complex dielectric function, $\operatorname{Re}[\varepsilon(\omega)]$, is negative in the visible spectrum and passes through the $\operatorname{Re}[\varepsilon(\omega)]=0$ condition still in the interband spectral region. The epsilon near zero condition (ENZ) defines the bulk plasmon frequency of a solid-state plasma and the frequency range in which the free electrons can respond collectively to screen external electromagnetic fields.[1] The bulk plasmon frequency at 3.8-3.9 $\mathrm{eV}$ defines also the surface multipole and monopole plasmonic responses at dielectric/Ag interfaces.[2] In this thesis, by employing interferometric time-resolved multi-photon photoemission electron microscopy (ITR-mP-PEEM), I study the interfacial, collective plasmonic fields where light is spatially and temporally localized on nano-femto scales in surface plasmon polaritons (SPPs) modes of nanostructured Ag metal films and domains. PEEM records the surface electromagnetic fields by imaging the spatial photoelectron distributions generated by their nonlinear interactions. I employ the ITR-mP-PEEM instrument to image the capture, propagation and interference phenomena of the SPP fields by recording ultrafast microscopic movies of their space and time evolution. With the understanding of the excitation mechanism and the dynamics

of the locally confined SPP field, we gain the fundamental knowledge of photon induced physical phenomena on the nano-femto scale. 


\subsection{Collective Electron Oscillations}

\subsubsection{Plasma Model}

The optical response of free electron plasmas in form of metals is well described by Maxwell's equations.[3] When interacting with matter, the electric field $\boldsymbol{E}$ and the magnetic induction $\boldsymbol{B}$ are linked to the dielectric displacement $\boldsymbol{D}$ and the magnetic field $\boldsymbol{H}$ via the polarization $\boldsymbol{P}$ and the magnetization $\boldsymbol{M}$ by

$$
\begin{gathered}
D=\varepsilon_{0} E+P \\
H=\frac{1}{\mu_{0}} B-M
\end{gathered}
$$

In linear, isotropic, and nonmagnetic media, we can define the above properties as:

$$
\begin{aligned}
& D=\varepsilon_{0} \varepsilon E \\
& B=\mu_{0} \mu H
\end{aligned}
$$

where $\varepsilon$ is the dielectric constant or relative permittivity and $\mu$ is the relative permeability of materials considered.

Next, we consider noble metals and describe their optical properties as a free electron gas plasma, or the Drude model.[4] In this case, details of the lattice potential, electron-electron, electron-ion, and electron-phonon interactions are not considered. Instead, the model is based on the simplest free-electron band structure, which defines the electron mass and density. When an electromagnetic field is applied, electrons are accelerated and oscillate in response to the applied field, but their motion becomes randomized with a characteristic collision, or damping frequency

$\gamma_{\text {damp }}=\frac{1}{\tau_{\text {relax }}}$, where $\tau$ is the momentum scattering time of the free electron gas. For typical metals at $300 \mathrm{~K}, \tau_{\text {relax }}$ is on the order of $10 \mathrm{fs}$. One should notice, however, that this is a simplified 
model, which does not apply to real metals when excited by visible light because the interband and intraband excitation of electrons can have important roles.[5]

When a time dependent electric field is applied to a metals, it is instructive to consider the equation of electron motion based on a damped harmonic oscillator model,

$$
m_{e} \ddot{x}+m_{e} \gamma_{d a m p} \dot{x}=-e E(t)
$$

where the driving field $\boldsymbol{E}(t)$ is assumed to have harmonic time dependence, $\boldsymbol{E}(t)=\boldsymbol{E}_{0} \mathrm{e}^{-\mathrm{i} \omega \mathrm{t}}$ and $\boldsymbol{m}_{\boldsymbol{e}}$ is the free electron mass. The solution of electron motion is

$$
x(t)=\frac{e}{m_{e}\left(\omega^{2}+i \gamma_{d a m p} \omega\right)} E(t)
$$

The electron motion describes displacement with respect to the ion background by $\boldsymbol{x}(t)$, which will contribute to the macroscopic polarization field $\boldsymbol{P}=-n e \boldsymbol{x}$, where $\mathrm{n}$ is the electron density. Therefore, one can write the displacement field using Eqs. 1.1 as

$$
D=\varepsilon_{0} E-\frac{n e^{2}}{m_{e}\left(\omega^{2}+i \gamma_{d a m p} \omega\right)} E=\varepsilon_{0}\left(1-\frac{\omega_{p}^{2}}{\omega^{2}+i \gamma_{d a m p} \omega}\right) E
$$

where $\omega_{p}^{2}=\frac{n e^{2}}{\varepsilon_{0} m_{e}}$ is the plasma frequency of the free electron gas. The dielectric function of the free electron gas can be described the Drude model, which assumes that the electrons experience only a uniform positive background, and is given by

$$
\varepsilon(\omega)=1-\frac{\omega_{p}^{2}}{\omega^{2}+i \gamma_{d a m p} \omega}
$$

The excitation at frequency $\omega_{p}$ corresponds to the collective excitation of the free electron gas oscillating against the fixed positive ion background. A schematic picture of the electron displacement and local charge distribution of the free electron gas and the ionic background is shown in Figure 1.1, where the plasma is excited by electron bombardment, as indicated by the arrow. 


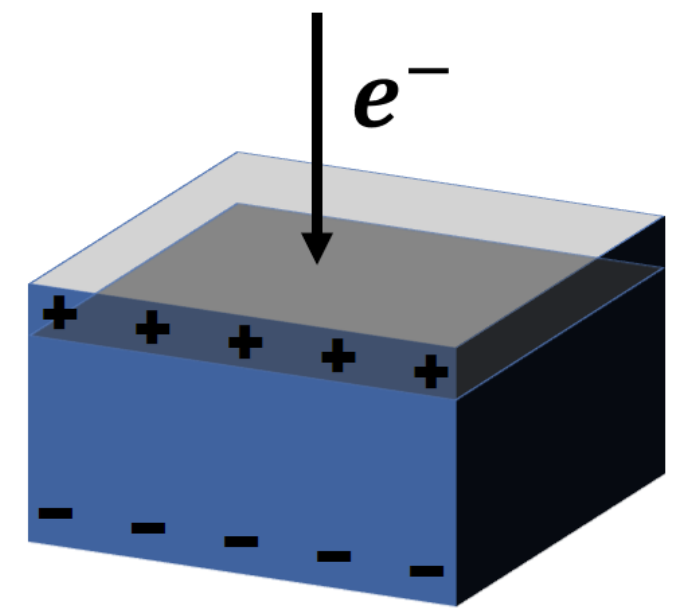

Figure 1. 1 Schematic of bulk plasmon charge oscillation.

\subsubsection{Localized Surface Plasmons (LSPs)}

When metals exist in the form of nanoscale particles, which have the size dimensions $d$ that are much smaller than the wavelength of the excitation light, i.e. $d \ll \lambda$, conduction electrons can be excited to oscillate collectively within the particle. Unlike a bulk plasmon, which can propagate through a solid, this extremely confined collective excitation can be described by a simple quasi-static approximation method, where the phase of the harmonic driving field is constant over the particle size. [4] 


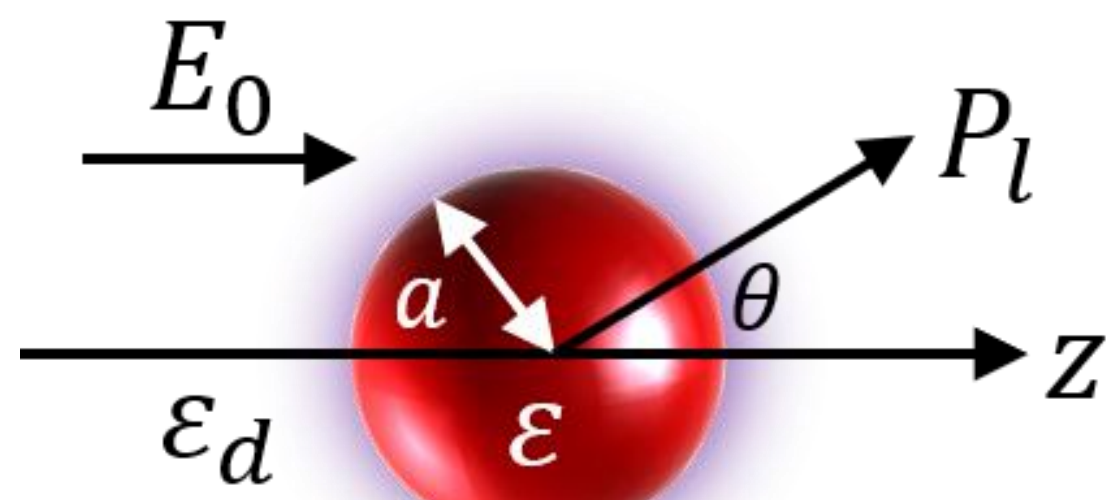

Figure 1. 2 Schematic of a localized surface plasmon in a spherical particle.

The simplest structure to consider for an LSP mode is a homogeneous, isotropic sphere of radius $a$, that is placed in an isotropic, non-absorbing dielectric medium, with a dielectric constant $\varepsilon_{d}$, as shown in Figure 1.2. When a static electric field is applied in the positive $\mathrm{z}$ direction, the electric field can be described as $\boldsymbol{E}=E_{0} \hat{\boldsymbol{z}}$. The interaction of an electric field with the spherical particle is described by solving the Laplace equation for the potential $\nabla^{2} \phi=0$, where $\phi$ is the electric potential and $\boldsymbol{E}=-\nabla \phi$. The standard solution of the Laplace equation giving the electric potential under certain boundary conditions, has the following form,

$$
\phi(r, \theta)=\sum_{l=0}^{\infty}\left[A_{l} r^{l}+B_{l} r^{-(l+1)}\right] P_{l}(\cos \theta)
$$

where $P_{l}(\cos \theta)$ are the Legendre Polynomial of the order $l$, and $\theta$ is the angle between the position vector $\boldsymbol{r}$ along direction $\boldsymbol{P}_{\boldsymbol{l}}$ and the $z$-axis. By applying boundary conditions at the surface of the sphere and the finite field requirement at the particle center, the potential outside of the particle can be described as 


$$
\begin{gathered}
\phi_{o u t}=-E_{0} r \cos \theta+\frac{p \cdot r}{4 \pi \varepsilon_{0} \varepsilon_{d} r^{3}} \\
p=4 \pi \varepsilon_{0} \varepsilon_{d} a^{3} \frac{\varepsilon-\varepsilon_{d}}{\varepsilon+2 \varepsilon_{d}} E_{0}
\end{gathered}
$$

where $\boldsymbol{p}$ is defined as the dipole moment of the particle. I further introduce the polarizability $\alpha$, where $\boldsymbol{p}=\varepsilon_{0} \varepsilon_{d} \alpha E_{0}$, to obtain

$$
\alpha=4 \pi a^{3} \frac{\varepsilon-\varepsilon_{d}}{\varepsilon+2 \varepsilon_{d}}
$$

For a metallic particle with dielectric function $\varepsilon(\omega)$ described by the Drude model, the polarizability of a particle experiences a resonance when the $\left|\varepsilon+2 \varepsilon_{d}\right|$ approaches a minimum. Because the dielectric function of metal is negative in the intraband absorption region, and that of its dielectric environment is positive, the particle must have an environment-dependent resonance. In this case, the resonance is called a localized surface plasmon resonance (LSPR)[6] and the associated mode is called the dipole surface plasmon of the particle.

Note that this description is only valid when the particle size is extremely small. When the particle size becomes comparable to the wavelength of excitation, the quasi-static approximation is no longer valid, and one must account for the retardation effect across the particle, which considers that the electric field is no longer uniform across the particle, but has a phase gradient. In such case, a rigorous electrodynamic description must be applied. In 1908, Mie developed a complete theory, which solves the scattering and absorption properties of colloidal particles that are illuminated by light. This rigorous approach is known as the Mie theory, which provides a description of light scattering by a particle in a set of normal modes. The quasi-static solution is the first, and usually dominant term of the complete expansion in Mie solution. [7-9] 


\subsubsection{Surface Plasmon Polaritons (SPPs)}

When the collective oscillating modes are excited locally at metal surfaces, they can propagate along a metal/dielectric interface. Such propagating modes are defined as surface plasmon polaritons, or SPPs in short. The propagating modes take form of an electromagnetic wave on the dielectric side and electron (charge) density wave in the metal.

To describe such propagating mode, we solve the Maxwell's equations at metal/dielectric interface with the correct boundary conditions. The corresponding wave equation for the electric field, where it is assumed to have a harmonic time dependence, is as follows,

$$
\nabla^{2} E+k_{0}^{2} \varepsilon E=0
$$

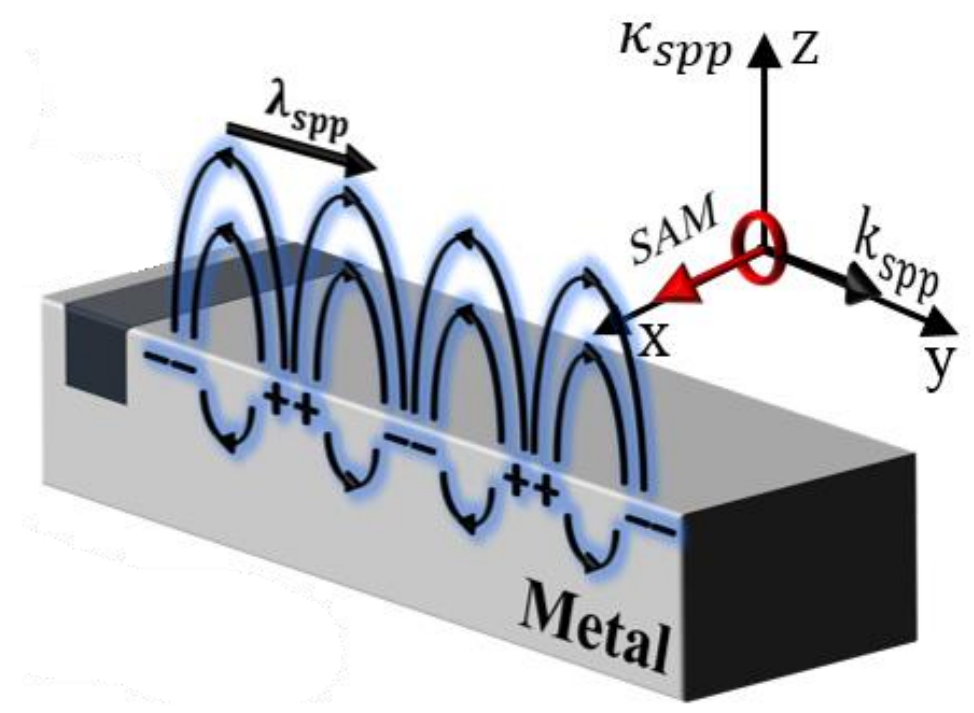

Figure 1. 3 Schematic of the surface plasmon polaritons at metal/vacuum interface. 
Here $k_{0}=\frac{\omega}{c}$ is the wave vector of the propagating mode in vacuum. Consider the geometry in Figure 1.3, where the wave propagates in the y direction. The evanescent wave solution for the above equation in the dielectric part is the following,

$$
\begin{gathered}
H_{x}(z)=A_{2} e^{i \beta y} e^{-k_{2} z} \\
E_{y}(z)=i A_{2} \frac{1}{\omega \varepsilon_{0} \varepsilon_{2}} k_{2} e^{i \beta y} e^{-k_{2} z} \\
E_{z}(z)=-A_{2} \frac{\beta}{\omega \varepsilon_{0} \varepsilon_{2}} e^{i \beta y} e^{-k_{2} z}
\end{gathered}
$$

And the solution for the metal part is,

$$
\begin{gathered}
H_{x}(z)=A_{1} e^{i \beta y} e^{-k_{1} z} \\
E_{y}(z)=i A_{1} \frac{1}{\omega \varepsilon_{0} \varepsilon_{1}} k_{1} e^{i \beta y} e^{-k_{1} z} \\
E_{z}(z)=-A_{1} \frac{\beta}{\omega \varepsilon_{0} \varepsilon_{1}} e^{i \beta y} e^{-k_{1} z}
\end{gathered}
$$

where $\beta$ is the complex wave vector along the interface, $k_{i}(i=1,2)$ represent the $\mathrm{z}$ component of the wave vector normal to the interface, and $\varepsilon_{1}$ and $\varepsilon_{2}$ are the dielectric constants for the metal and dielectrics respectively. Note that the boundary conditions prescribed by the Maxwell's equations require that the $H_{x}$ and $\varepsilon E_{y}$ fields at the metal/dielectric (vacuum) interface are continuous, therefore $k_{1} \varepsilon_{1}=-k_{2} \varepsilon_{2}$. Thus, a propagating mode is supported when the real part of the dielectric constant is negative for metal $\left(\operatorname{Re}\left(\varepsilon_{1}\right)<0\right)$ and positive for dielectric $\left(\operatorname{Re}\left(\varepsilon_{2}\right)>0\right)$. Therefore, based on the continuity condition at the interface, we obtain the dispersion relation of the propagating mode at metal/dielectric interface to be,

$$
\beta=k_{0} \sqrt{\frac{\varepsilon_{1} \varepsilon_{2}}{\varepsilon_{1}+\varepsilon_{2}}}
$$


The dispersion of the SPPs mode at silver/vacuum interface is plotted in Figure 1.4, where the frequency axis is normalized by the bulk plasmon frequency $\omega_{p}$. The blue line represents the light line in free space, which satisfies the relation $\omega=c k$. The red line is the surface plasmon (SP) the $k \rightarrow \infty$ asymptote of the SPP mode. For Drude, i.e., free-electron metal/vacuum interface, the SP frequency is $\omega_{S P}=\omega_{S P} / \sqrt{2}$. The SP frequency depends on the dielectric environment and can more generally be expressed as $\omega_{s p}=\omega_{p} / \sqrt{1+\varepsilon_{2}}$, where $\varepsilon_{2}$ is the dielectric constant of the dielectric. The dotted line represents the experimental dispersion function of the SPPs mode, taken from an ellipsometric measurements of Ag/vacuum interface from Johnson and Christy. [1]

Because of the matter wave character, the SPPs dispersion line lies to the right of the free space light line. Therefore, for a perfectly flat surface, the momentum mismatch prevents the direct excitation of SPPs unless momentum can be supplied by, for example, a surface asperity, which supports a localized plasmon mode. For such a localized plasmon mode, the plasmon has a broad spectrum of momenta, enabling it to decay into accessible propagating modes.[10]

As the wave vector increases, the SPPs dispersion moves away from the light line till the frequency approaches to the surface plasmon frequency, $\omega_{s p}$, and the mode takes on more of charge density character. This causes it to couple more strongly to single particle excitations, causing the SPP mode dissipation.

The SPP propagation or decay length is expressed by the imaginary part of the effective dielectric function. For a perfectly flat silver/vacuum interface, the propagation length defined as $L=[2 \operatorname{Im}(\beta)]^{-1}$, falls in the range from the vacuum $\sim \lambda_{s p p}$ when the energy of the excitation light approaches $\omega_{s p}$, to $\sim 100 \mu \mathrm{m}$ in the long wavelength limit.[4, 10] 


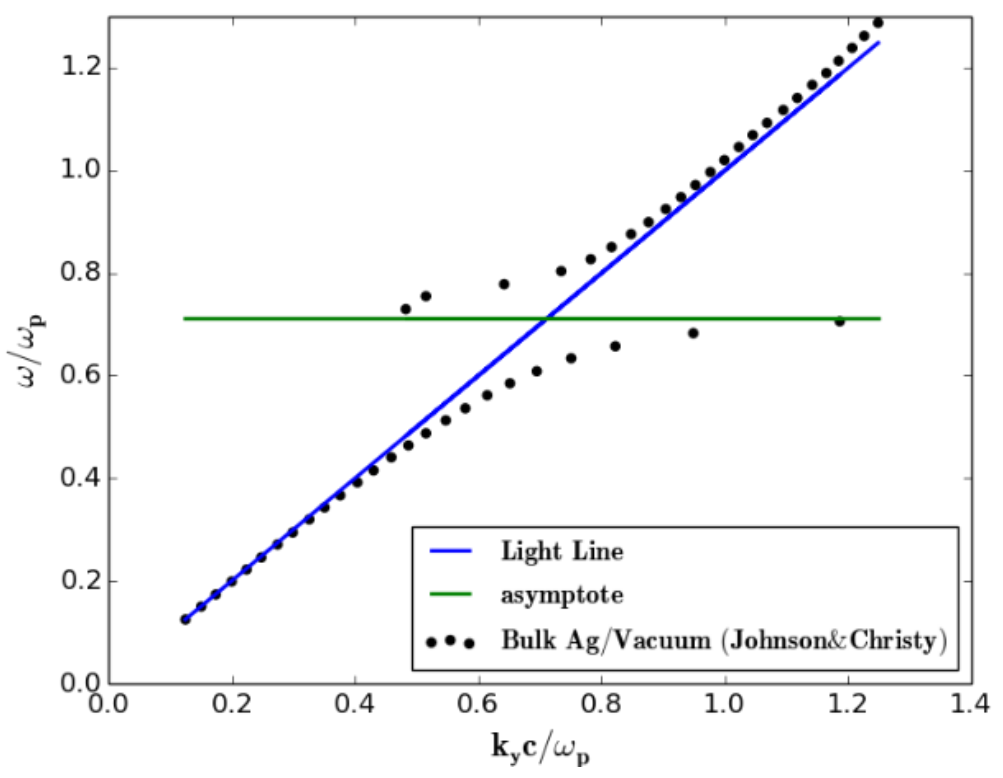

Figure 1. 4 Dispersion relation of SPPs at Ag surfaces.

A key feature of surface evanescent waves such as SPPs, which is a part of my research, is that they carry non-zero transverse spin-angular momentum (SAM) independent of the SAM of light that generates them.[11-16] The field in Eq 1.15 describes a transversely polarized SPPs plane wave. Based on that, if we consider a general case of SPPs, where the polarization vectors contain all three cartesian components, then the $\boldsymbol{E}$ field should be expressed as follows,[11]

$$
E(r)=\frac{A \sqrt{\mu}}{\sqrt{1+|m|^{2}}}\left(m_{p} \beta \widehat{x}+i k_{z} \widehat{y}-k_{y} \hat{z}\right) e^{\left(i k_{y} y-k_{z} z\right)}
$$

Here $\boldsymbol{\mu}$ is the permeability of the material, $\beta=\boldsymbol{k}_{\boldsymbol{y}} \hat{\boldsymbol{y}}+\boldsymbol{i} \boldsymbol{k}_{\mathbf{z}} \hat{\boldsymbol{z}}$ is the complex k-vector of SPPs, and $\boldsymbol{m}_{\boldsymbol{p}}$ is a complex number that defines the polarization of light [11],

$$
\tau=\frac{1-\left|m_{p}\right|^{2}}{1+\left|m_{p}\right|^{2}}, \quad \chi=\frac{2 \operatorname{Re}\left(m_{p}\right)}{1+\left|m_{p}\right|^{2}}, \quad \sigma=\frac{2 \operatorname{Im}\left(m_{p}\right)}{1+\left|m_{p}\right|^{2}}
$$


where $\tau$ describes the polarization component in the $x / y$ directions, $\chi$ describes the polarization component along $+45^{\circ} / 45^{\circ}$ directions with respect to the $x$ direction, and $\sigma$ describes the helicity of light, where \pm 1 represent left/right circularly polarized light. Consider the energy flow of such SPP waves defined by their Poynting vector, $\boldsymbol{p}=\frac{g}{2 c} \operatorname{Re}\left(\boldsymbol{E}^{*} \times \boldsymbol{H}\right)$, where $g=\frac{1}{4 \pi}$ in the Gaussian units. The Poynting vector can be decomposed into two terms as $\boldsymbol{p}=\boldsymbol{p}^{o}+\boldsymbol{p}^{s}$, where $\boldsymbol{p}^{o}$ represents the observable, canonical or orbital angular momentum (OAM) density, which accounts for the energy transport. By contrast, $\boldsymbol{p}^{S}$ represents the spin angular momentum (SAM) of light that does carry energy, and is defined by the spin angular momentum density $\boldsymbol{s}$ via the relation,

$$
p^{s}=\frac{1}{2} \nabla \times s,
$$

where

$$
s=\frac{g}{4 \omega} \operatorname{Im}\left[\mu^{-1}\left(E^{*} \times E\right)+\varepsilon^{-1}\left(H^{*} \times H\right)\right]
$$

Note that for linearly polarized light in free space, $\boldsymbol{s}$ vanishes because it only has a transverse electric field along one direction, for which the cross-product is zero. For circularly polarized light, Eq. 1.20 obtains a spin angular momentum, with the direction that is either parallel or anti-parallel to the light wave vector. Now by inserting the electric field defined in Eq. 1.17 and the corresponding magnetic field into Eq. 1.19, we arrive at the following expression for $\boldsymbol{p}^{s}$,

$$
p^{s}=\frac{w}{\omega n^{2}}\left(\frac{k_{z}^{2}}{k_{y}} \widehat{y}+\sigma \frac{k_{z} k}{k_{y}} \widehat{x}\right)
$$

where $k^{2}=k_{y}^{2}-k_{z}^{2}$. As we can see, the SAM contains not only component in the direction of propagation, but also a transverse SAM orthogonal to the wave vector. In addition, for a transversely polarized plane SPP wave, the spin vanishes in the $\boldsymbol{y}$ direction; thus only the transverse component of the spin remains. Besides, the direction of the transverse SAM (the $\widehat{\boldsymbol{x}}$ in 
Eq. 1.21) is dependent on the Stokes parameter $\sigma$,which has the opposite sign for counterpropagating SPP waves, thus counter-propagating SPP waves have opposite transverse spin direction; this is the basis for the terminology of "the plasmonic quantum spin Hall effect," in analogy to the quantum spin Hall effect, though in reality it is a purely classical property of SPP waves, Nevertheless, this will be referred as the "plasmonic quantum spin Hall effect" according to custom and resemblance to the electron counterpart, though it should be understood that it is not a quantum effect. The experimental evidence of the transverse spin of SPPs will be discussed in Chapter 5.

\subsection{Techniques of Imaging Plasmon Related Phenomena}

Surface plasmons were first detected as electromagnetic modes of metal/dielectric interfaces by electron energy loss spectroscopy (EELS), where a loss feature appeared that could be attributed to them.[4] Subsequently, several other techniques have been developed that enable imaging of surface plasmons in real space, with demonstrated spatial resolution down to a few nanometers. The most commonly used technique is near field scanning optical microscopy (NSOM)[17-25], where a sharp tip scans across a sample surface and measures the locally scattered light at the scanning tip, induced by the SPP waves and the excitation light, which interacts with the sample surfaces. Such scanning techniques are limited by the fact that they record one location at a time, and therefore the scan rate and dimensions limit their capability for generating 2D images. Other techniques such as leakage radiation microscopy (LRM)[26, 27], fluorescence microscopy[28, 29], are also capable of imaging surface plasmon fields with tens of nanometer resolution. Combined with two-pulse interferometry, the mentioned methods can be 
used for imaging of the temporal evolution of plasmonic fields. These methods, however, suffer from serial data acquisition constraints, which limit their data acquisition rate, as well as perturbation by their probes of the optical near fields. Such limitations are solved by the interferometric time-resolved multiphoton photoemission electron microscopy (ITR-mPEEM)[3042], which will be introduced in the following sections.

\subsubsection{Photoemission Electron Microscopy (PEEM)}

PEEM employs photoemission of low energy electrons, which are spatially imaged with electron optics to reveal the surface electric fields, electronic properties, or material topography that determine the photoelectron spatial distributions. The simplest PEEM microscope consists of one electrostatic objective lens that collects photoelectrons and a microchannel plate (MCP) fluorescent screen as a detector. Prior to the invention of PEEM, electron optics for imaging with low energy electrons have been developed by Ernst Bauer, which has setup up the basis of the development of PEEM technique.[43-45] After significant improvements in the electron optics, advanced PEEM instruments, such as the instrument that I used at the University of Pittsburgh, with $<10 \mathrm{~nm}$ spatial resolution for photoelectrons with only of a few electron volts energy, could be achieved.[46-48] Sharing with the same electron imaging optics, a PEEM instrument is sometimes combined with an electron gun, which produces low energy electrons that can also be used for imaging sample surfaces. Such operation mode is referred as low energy electron microscopy (LEEM), where, instead of imaging photoelectrons, LEEM images the backscattered electrons, which carry information such as surface topography. All experiments in this thesis are based on the LEEM/PEEM instrument, with the details to be presented in Chapter 2. 
Our LEEM/PEEM instrument is operated with either a UV discharge lamp or a femtosecond laser source for exciting linear or non-linear photoemission. When the light source has photon energy higher than the work function of the sample, single photons have sufficient energy to excite electrons into the vacuum. The photoelectron emission spatial distribution may depend on the material properties or structuring, which provide contrast in a PEEM image. If the sample is irradiated by a low photon energy light and from high-power femtosecond laser pulses, the work function of the sample may be too high for linear photoemission, however, a non-linear, two or more photon, photoemission process can take place when the total photon energy is sufficient to excite electrons to energies above the work function and thus be emitted into vacuum.[49-55] In such case, PEEM images may be dominated by the local field amplitude to the $2 n^{t h}$ power, where $\mathrm{n}$ is the photonic order of the photoemission process.[30-33, 40, 50, 56] The PEEM imaging signal can be expressed as,

$$
I_{P E E M}(x, y, t) \sim \int_{-\infty}^{t}\left(P_{t o t}\left(x, y, \tau^{\prime}\right)\right)^{2 n} d \tau^{\prime}
$$

where $\boldsymbol{P}_{\text {tot }}$ is the total polarization field induced at the sample surface; the integration is over the total interaction time between the excitation pulse and the surface fields, because the photoelectron detection is slow. Figure 1.5(a) shows a schematic figure of a typical PEEM experiment, where an incident laser pulse irradiates the sample at an oblique angle of $70^{\circ}$ with respect to surface normal. Upon excitation, an electron acquires the energy of one or more photons so that it can be photoemitted into vacuum to form a PEEM image. In addition, light pulse illumination from surface normal direction is also available, which will be discussed in Chapter 6 along the preliminary experimental results. Typical PEEM image corresponding to the schematic is shown in Figure 1.5(b), which shows photoemission due to strong coupling of the propagating surface plasmon polaritons on a silver nanowire epitaxially grown on a $\mathrm{Si}(001)$ substrate. The external 
light illuminates the whole field of view of the PEEM, so that it is considered as a plane wave illumination. SPP fields are coupled at nanoscale surface obstacles because of the momentum scattering that conserve the total momentum[4]; In the case of the wire excitation (Chapter 4), the whole wire can be a source of SPP fields, while in the case of a 2D island (Chapter 4 and 5), all edges of the islands act as coupling sources for SPPs . However, in the wire excitation, because of the finite lateral dimension of the wire $(\sim 200 \mathrm{~nm})$, the SPP waves that can only propagate along the wire long axis. For 2D island excitation, SPP waves from all edges superimpose to on the surface to form PEEM images. The reason SPP fields are only coupled at nanoscale obstacles ( wire ends or island edge etc.) is that the external field can be scattered to contain a continuous momentum distribution, and part of the scattered momentum will match the momentum of SPP fields, to conserve the total momentum.

The photoemission signal consists of a periodic modulation, which we refer to as a surface plasmon polariton interference beating pattern. This beating pattern is stationary and depends on the time structure of the excitation light; It records differences in the local speeds of the excitation light and the SPP pulse that it generates propagate. Because at a given frequency the SPPs always has a larger wave vector, $\boldsymbol{k}_{s p p}=\operatorname{Re}\left(\widetilde{\boldsymbol{k}}_{s p p}\right)$, than that of light, an interference pattern between the two fields forms at the difference between the in-plane k-vectors of the excitation field, $\boldsymbol{k}_{L}$ and the SPP field, $\boldsymbol{k}_{s p p}$. This difference defines the $k$-vector of the beating pattern as $\boldsymbol{k}_{B}=\boldsymbol{k}_{s p p}-\boldsymbol{k}_{L}$, thus resulting the beating periodicity, $\lambda_{B},[35]$

$$
\lambda_{B}=\frac{\lambda_{L} \lambda_{s p p}}{\sqrt{\lambda_{L}^{2}+\lambda_{s p p}^{2}-2 \lambda_{L} \lambda_{s p p} \cos \gamma}}
$$


where $\lambda_{L}=\frac{2 \pi}{\boldsymbol{k}_{L}}=\lambda / \sin \theta$ is the in-plane projection of the excitation wavelength for the angle of incidence, $\theta, \lambda_{s p p}=\frac{2 \pi}{\boldsymbol{k}_{s p p}}$ is the SPP wavelength, and $\gamma$ is the in-plane angle between $\boldsymbol{k}_{L}$ and $\boldsymbol{k}_{s p p}$. In the case of wire excitation, $\boldsymbol{k}_{L}$ and $\boldsymbol{k}_{s p p}$ are either parallel or anti-parallel, thus $\gamma=0$, while in the case of island excitation [Figure 4.8 in Chapter 4], $\gamma$ is the angle between $\boldsymbol{k}_{\text {in }}$ and $\boldsymbol{k}_{\text {spp }}$.

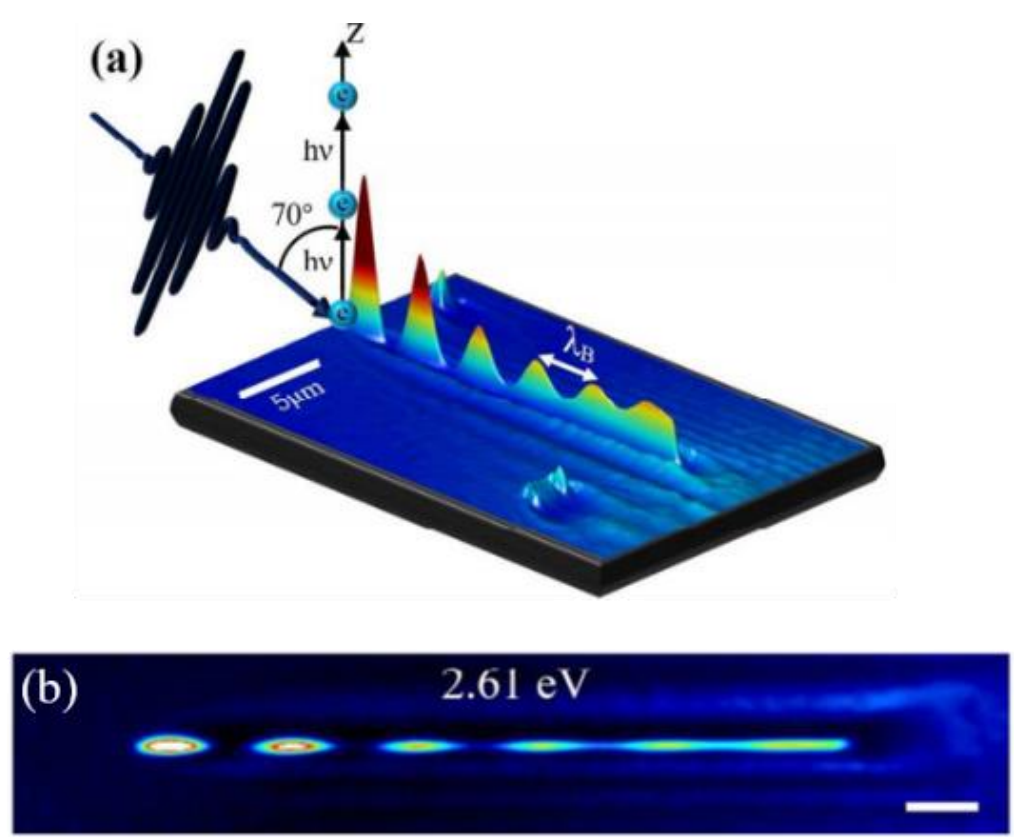

Figure 1. 5 (a) Schematic of oblique incident PEEM setup. (b) two-photon photoemission PEEM images of SPPs on Ag hv=2.61 eV. Scale bar is $2 \mu \mathrm{m}$.

By employing a Mach-Zehnder interferometer [Section 2.3.3], we can generate a phaselocked pump probe pulse pair to perform time-resolved (interferometric two pulse correlation, I2PC) PEEM experiment [Section 4.2.2]. In such experiments, the SPPs launched from the pump pulse propagate in space and time along metal surfaces. After the pump pulse is gone ( $25 \mathrm{fs})$, the 
probe pulse arrives, and interfere with the SPP fields launched by the pump pulse, to give photoemission together. As the delay between pump and probe pulse is scanned, we obtain the dynamical evolution of the SPP fields launched by the pump pulse.

\subsubsection{Low Energy Electron Microscopy (LEEM)}

In addition to imaging of surface plasmon fields using photoemission electron microscopy, a complementary technique, which allows us to image the geometry of the plasmonic materials, is also crucial for us to understand the plasmonic excitations. Low energy electron microscopy (LEEM), is ideally suited for this because it shares the same imaging electron optics as the PEEM.[44-47] Unlike the PEEM, where photoelectrons are used to image plasmonic fields, in LEEM, the electrons are provided by a field emission gun and are decelerated into the sample to few eV kinetic energies; the elastically back scattered low energy electrons are collected to image the geometry of the plasmonic materials, with a specified spatial resolution of $\sim 2 \mathrm{~nm}$. Such high spatial resolution can be obtained by aberration correction of the imaging electron optics.[46, 47] Because the illumination electrons are decelerated to a low energy, they do not penetrate deep into the sample surface. Instead, most electrons will be reflected by the top few atomic layers of a solid surface, thus making LEEM a very sensitive tool for surface imaging.[44, 45, 57, 58]

One imaging contrasts of LEEM is the interference among the reflected electrons due to the surface topography.[44] When electrons are reflected from different surface terrace that differ by an integer number of atomic height, due to the difference in optical paths between adjacent terraces, a phase difference is produced at the objective lens, which is detected as an amplitude difference.[59, 60] A typical LEEM image of a pure $\operatorname{Si}(111)$ surface is shown in the top panel of 
Figure 1.6, where a clear triangular line contrast is observed. Such contrast is due to the Si surface reconstruction and atomic step contrast due reflected electron interference.

A unique advantage of LEEM is its "true imaging" mechanism, in which case the microscope images the entire field of view upon illumination by electrons.[44] Thus, it provides a very efficient way of imaging sample surfaces, compared to the raster scanning type microscopy, such as scanning electron microscopy (SEM) or atomic force microscopy (AFM). In Figure 1.6, we show slices from a movie of monolayer silver growth on the $\mathrm{Si}(111)$ surface, as observed in real time. Initially the $\mathrm{Si}(111)$ surface shows its characteristic triangular contrast. Upon $\mathrm{Ag}$ deposition, bright contrast starts to show up at the step edges of $\mathrm{Si}(111)$ surface. As deposition continues, more Ag atoms are adsorbed on the surface, until the whole Si surface is covered by a monolayer of silver. With further Ag adsorption, 3D Ag clusters start to form through the StranskiKrastanov growth mode, Which occurs when Ag atoms are able to rapidly diffuse on the surface to grow triangular or rectangular single-crystal islands. Such growth is used to prepare single crystal plasmonic micro particles; imaging of their SPPs will be discussed in later chapters. 


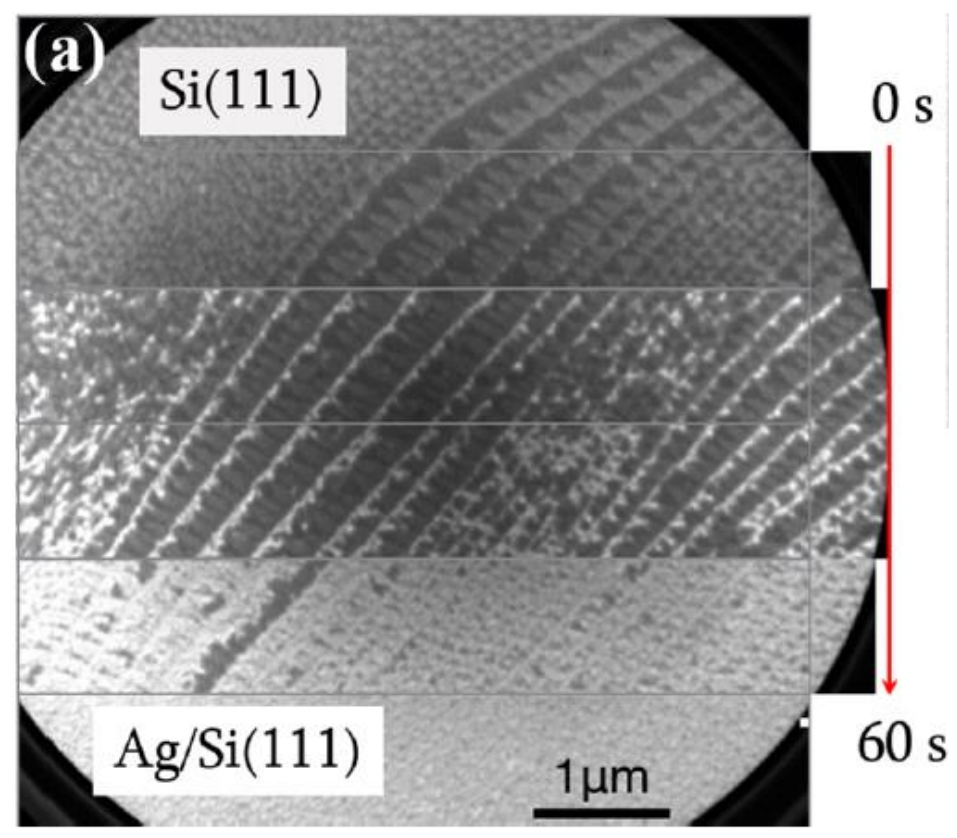

Figure 1. 6 LEEM image of Si(111) surface at sequential Ag coverage.

\subsubsection{Low Energy Electron Diffraction (LEED)}

When low energy electrons impinge on the surface of a crystalline structures, each atom of the sample surface act as a point scattering source of electron waves. The electron wavelength is given by the de Broglie equation $\lambda=\frac{h}{p}=\frac{h}{\sqrt{2 m e V}}$, in terms of the inverse of the electron momentum $p$. Here, $\lambda$ can be further related with the electron energy $\mathrm{eV}$, which shows that the larger the energy is, the shorter the wavelength an electron becomes. In a typical LEED experiment, the electron energies used are between $5 \sim 200 \mathrm{eV}$, corresponding to electron wavelength between 0.9 $\sim 5.5 \AA$. Thus, such electron wavelength range is comparable with the crystal lattice constants and is therefore, useful for determining the surface crystalline structure.[44, 45] 
Consider a 1-D chain of atoms, as shown in Figure 1.7(a). When electron waves are normally incident on the chain, they can be scattered at all angles by the surface lattice. At a particular scattering angle $\theta_{L E E D}$, scattered electrons will constructively interfere under the Bragg condition, where $\operatorname{asin}\left(\theta_{L E E D}\right)=n \lambda$. In a $2 \mathrm{D}$ periodic lattice case, the scattered pattern will form the LEED pattern in the form of a $2 \mathrm{D}$ reciprocal space, showing the information about the surface periodic lattice, in spatial units derived from the incident electron wavelength. A typical LEED pattern from clean $\mathrm{Si}(001)$ surface is shown in Figure 1.7(b), which is obtained by acquiring electron imaging in the back focal plane of the electron optics while operating in the LEEM mode. One unit cell is composed of either $2 \times 1$ or $1 \times 2$ lattice reconstruction, which are labeled in the LEED image for reference. Both types reconstructed terraces contribute identically to the reflected electrons because of their rotational symmetry.
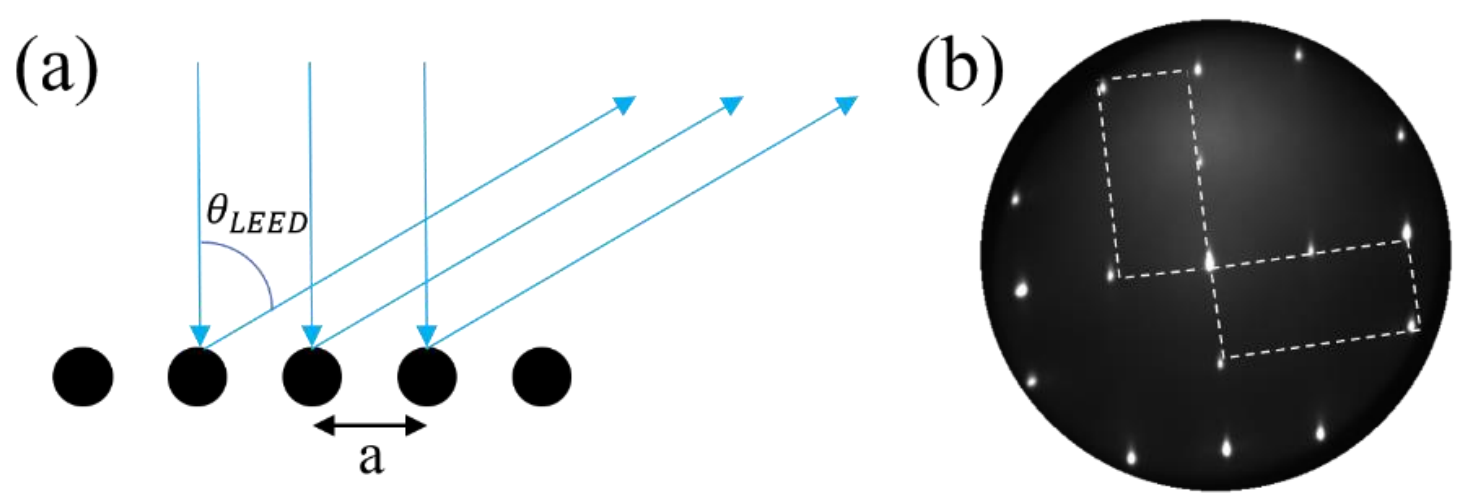

Figure 1.7 (a) 1-D chain schematic of electron scattering (b) 2-D LEED pattern of clean Si(001) surface. 


\subsection{Experimental Methods}

To study plasmonic phenomena and their dynamics, apparatus which can spatially and temporally resolve surface plasmon fields is required. We have an aberration corrected low energy electron microscopy/photoemission electron microscope (AC-LEEM/PEEM), commercially available as FE-LEEM P90 from Specs GmbH, to spatially resolve the plasmon fields, with specified resolution of about $8 \mathrm{~nm}$. [46, 47] The AC-LEEM/PEEM instrument is combined with a broadly tunable femtosecond pulsed laser system, which produces $20 \sim 25$ fs duration pulses, at a repetition rate of typically $1 \mathrm{MHz}$ in the $\sim 270-900 \mathrm{~nm}$ range. By combining time resolved pump-

probe experiment with delay scanning time resolution of about $0.1 \mathrm{fs}$, we are able to temporally resolve the plasmon dynamics, typically evolve with the phase and group velocities of SPPs.

\subsection{UHV Components of LEEM/PEEM}

\subsubsection{Cold Field Electron Emission Gun}

In the LEEM mode, the electron source for imaging is a cold field emission gun. A schematic of the electron gun working principle is shown in Figure 2.1. Typically, the electron emitter is a single crystal tungsten tip, with a radius of around $100 \mathrm{~nm}$. By applying a high voltage of $15 \mathrm{kV}$ in the case of current LEEM setup, a $15 \mathrm{keV}$ electron beam is extracted through the field emission process with an energy spread of about $0.3 \mathrm{eV}$. This energy spread determines the coherence, and to a significant degree, the resolution that may be obtained with LEEM. The emitter 
requires an ultrahigh vacuum in the low $10^{-10}$ mbar range. A valve isolates the gun from the rest of the chamber when the gun is not in use, thus maintaining good pressure in the gun chamber.

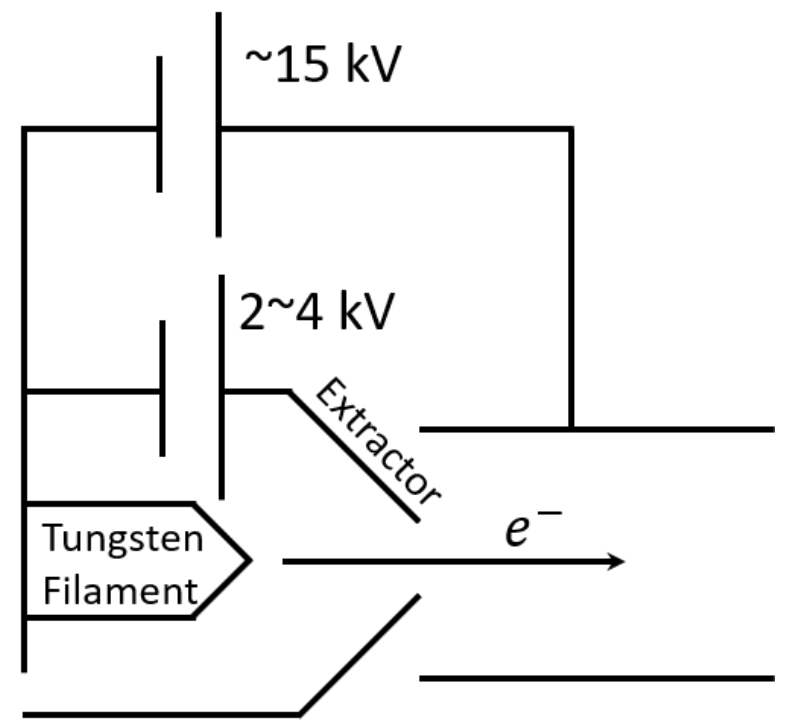

Figure 2. 1 Schematic of the cold field emission gun of FE-PEEM P90 system.

\subsubsection{Electron Optics}

Figure 2.2 display a schematic of the electron optical system of FE-LEEM P90 that enables microscopy. Here, I briefly explain how electrons are formed and processed by the optics. As mentioned before, in the LEEM system, electrons are emitted from the cold field emission gun and accelerated to $15 \mathrm{keV}$ potential and subsequently their paths are deflected by magnetostatic lenses. Electrons pass through a magnetic gun lens and a condenser lens, which vary the magnification of the whole illumination system and focus the electron beam at the entrance plane of a magnetic 
prism array (MPA). Two MPA systems are labeled as MPA1 and MPA2 in the figure. The MPA1 deflects the electron beam by 90 degrees and refocuses it in a symmetrically located exit plane in the objective lens. A transfer lens, M1, again focuses the beam in the back focal plane of the cathode objective lens. After deceleration, a well-defined, collimated low energy electron beam (typically of a few eV) impinges onto the sample. On a crystalline sample, the low energy electrons can experience diffraction, and after reflection and re-acceleration by passing through MPA1 once again, a low energy electron diffraction (LEED) pattern is formed at the back focal plane of the objective lens. This is the reciprocal plane of the imaging system. The objective lens can also form a real-space image of the sample at a longer distance.

In a PEEM experiment, photoemitted electrons replace the backscattered electrons as source of imaging. The photoemission source can be either high power UV lamp, or femtosecond laser pulse. The incidence angle of the light source can be set to $70^{\circ}$ from surface normal, or $0^{\circ}$ for the normal incidence excitation. When electrons are photoemitted, an angle resolved photoemission spectrum forms at the back focal plane.

The transfer lens M1 focuses the LEED pattern at the entrance plane of the MPA1, where an energy filter is also placed. Such energy filter allows us to select low energy electrons at desired momentum and energy, so that a spectroscopic measurement of electrons can be obtained. A further magnified real-space image on the diagonal plane of the MPA1 is formed because of the transfer lens M1. The MPA1 again deflects the electron beam by 90 degrees. The LEED pattern in the exit plane of the MPA1 is placed in the center of a magnetic transfer lens between MPA1 and MPA2. 


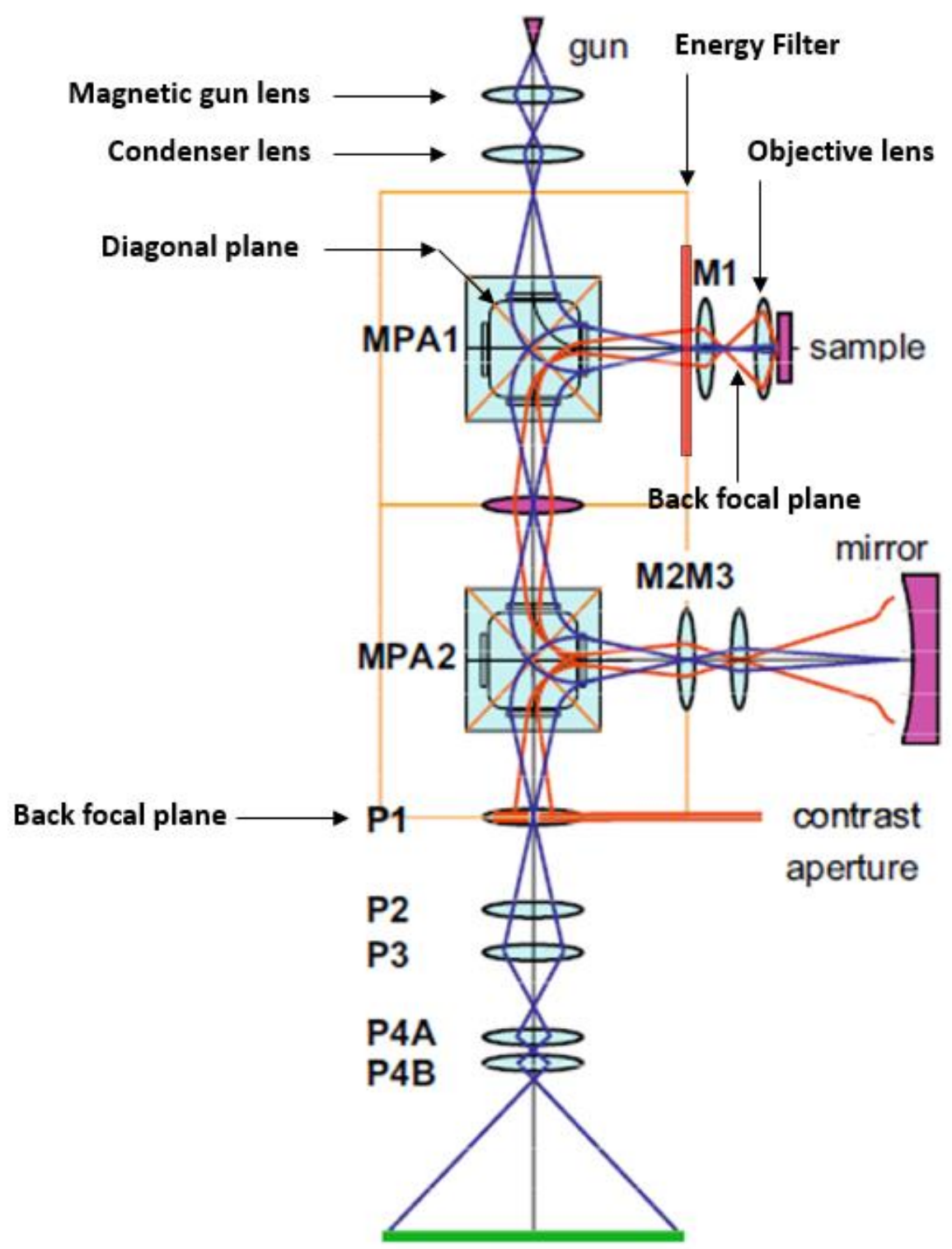

Figure 2. 2 Schematic of the major components of the electron optics in FE-PEEM P90 system.The red and blue paths indicate axial ray and field ray, respectiely.

Because electron optics such as MPA have chromatic and spherical aberration, they limit the resolution of electron microscopes. By symmetry, however, putting another MPA (MPA2) right after electrons exit the first one (MPA1), such aberration can be canceled. The aberration 
correction system is placed in-line with the electron optical system after electrons emerge from the first MPA. The aberration correction system consists of a set of electron mirrors consisting of a front lens that is held at the ground potential, and two ring-shaped elements at intermediate potentials $\mathrm{V} 3$ and $\mathrm{V} 2$, and a mirror electrode maintained at potential V1. By finding the best combination of $\mathrm{V} 1, \mathrm{~V} 2$ and $\mathrm{V} 3$, one is able to compensate both the spherical and chromatic aberration of the electron optics. After correction, the electron beam is reflected to the MPA2, where it is further deflected 90 degrees to the projection column.

\subsubsection{Projection Column}

When electrons enter the projection column, the first projector lens, $\mathrm{P} 1$, takes the sample image located in the diagonal plane of the MPA2 and reimages it in front of P3 for further magnification. The second projector lens, $\mathrm{P} 2$, is used to switch imaging between the real space and k-space, by applying a desired current as trigger. If not activated, P1 will transfer the real image from the MPA2 diagonal to the object plane of P3. If P2 is held at pre-defined current, it will transfer the diffraction from the contrast aperture plane to the P3 object plane. Next, P3 magnifies the image or diffraction pattern present in its object plane into the object plane of the final projector lens P4. Its magnification can be adjusted over a large range. The final projector lens $\mathrm{P} 4$ consists of two, identical, closely coupled lenses (P4a and P4b). At low excitation, when the focal length of each is equal to the lens separation, the pair forms an upright image with low magnification. At high excitation, when each lens has its shortest focal length, the pair forms an inverted image with significantly larger magnification. The whole projection column runs at high power, and thus producing heat that thermalize the electrostatic lenses, and creating thermal drift in imaging. Therefore, water-cooled system is installed to maintain the system at operation temperature 
$\left(\sim 60^{\circ} \mathrm{C}\right)$ and prevent from damaging by heat. Finally, P4 projects the image onto a CCD camera, where it is recorded, digitized, and sent to the computer where it can be displayed on a screen.

\subsubsection{Apertures}

There are a number of apertures that regulate the electron beam at various points in the microscope. These can be moved using linear drives (screwing the drive inward/outward reduces/increases the aperture size).

A micro-diffraction aperture controls the size of the incoming electron beam through the prism to the sample; it is placed at 45 degree to the MPA1. Changing the size of the aperture changes the viewing area of the microscope. The built-in aperture sizes are 5, 10, 20, 50 and 100 microns.

A selected area aperture controls the size of the reflected electron beam that leaves the MPA1, it is placed on the lower side of the MPA1, and like micro-diffraction apertures, mounted at 45 degree to the MPA1. The built-in aperture sizes are 50, 100, 200 and 400 microns.

A contrast aperture can change the resolution of the detected image and also the acceptance angle from the sample. The built-in aperture sizes are 10, 20, 40 and $70 \mu \mathrm{m}$. Such aperture is placed at the exit of the MPA2 in the diffraction plane. This aperture needs to be selected according to application: for spectroscopy it determines the energy resolution, and for microscopy, the spatial resolution. The general setting rules are 1) With small apertures, diffraction effects of the electron beams at the aperture degrade the image. 2) Large apertures limit the spatial resolution through spherical aberration. From experience, the optimal value is $40 \mu \mathrm{m}$. Depending on the beam intensity, one may use larger or smaller apertures at the cost of resolution. 


\subsection{Sample Preparation}

\subsubsection{Sample Stage}

Samples in our LEEM/PEEM system are mounted in sample caps, which fit onto the end of the sample holder (gray), as shown in Figure 2.3. The sample holder contains a filament for electron beam bombardment heating of the sample from the back side, as indicated by the arrows. In order to allow heating while the sample is at $15 \mathrm{kV}$, the heating power supply floats at $15 \mathrm{kV}$. There are two electronic units used for sample heating, EBH 100L control unit and LPU-sample power supply. As standard, there is no temperature sensor attached to the sample. Although the sample holder does contain contacts that allow a thermocouple to be fitted, we do not have thermocouples because of the tight spacing between sample cap and the filament and in many cases an infrared thermometer and experience provide reasonable estimates and sufficient guidance.

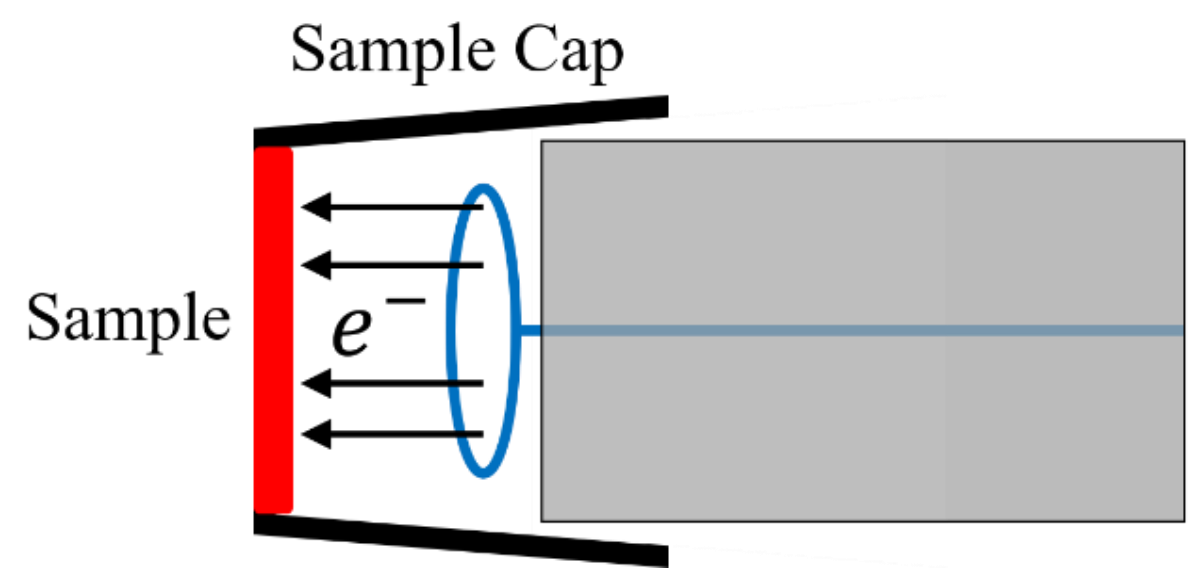

Figure 2. 3 Schematic of the sample stage in FE-PEEM P90 system. 


\subsubsection{Silicon Substrate Preparation}

Typical substrates used in LEEM/PEEM experiments are $\mathrm{Si}(001)$ and $\mathrm{Si}(111)$ surfaces. Prior introducing samples into LEEM/PEEM chamber, sample substrates are cleaned via standard Acetone, isopropanol (IPA), deionized water (DI water) cleaning procedure in an ultrasonic bath. After sample is introduced into ultrahigh vacuum, silicon substrates are heated to $\sim 800 \mathrm{~K}$ for several hours, to outgas the remaining water, $\mathrm{N} 2$ and $\mathrm{O} 2$ gases that remain on the substrates. Silicon is then further heated up to $\sim 1500 \mathrm{~K}$ for fast flashing (a few seconds) to remove the native oxide layer. Typically, we need to flash the substrate multiple times until we get clear LEEM image of a reconstructed Si surface. Typical LEEM images of $\mathrm{Si}(001)$ and $\mathrm{Si}(111)$ are shown in Figure 2.4. For $\mathrm{Si}(001)$, clean surface has two orthogonally reconstructed phases, i.e. $2 \times 1$ and $1 \times 2$ phases of the reconstructed Si atoms.[61-63] They have physically identical reflection properties, thus cannot be distinguished when electron beam is normally impinging on the surface. However, their LEED pattern in reciprocal space differ in orientations, thus by imaging the sample surfaces with electrons from only one LEED spot corresponding to a desired phase (using contrast aperture), we can image the surface reconstruction with LEEM such that the selected phase become bright and the rest become dark, and this operation mode is referred as dark field LEEM . For $\mathrm{Si}(111)$, the substrate forms triangular surface terraces, which result from the three-fold symmetry of the (111) orientation.[61] Such triangular terraces due to height difference, can be resolved in a normal operation LEEM mode, where electrons reflected from all angles are acquired, and this is referred as bright field LEEM image. Unlike in dark mode where only one reconstructed phase is 
bright, the contrast in bright field LEEM mode is mainly due to the low energy electron interference, which is modified by the height difference at atomic steps.
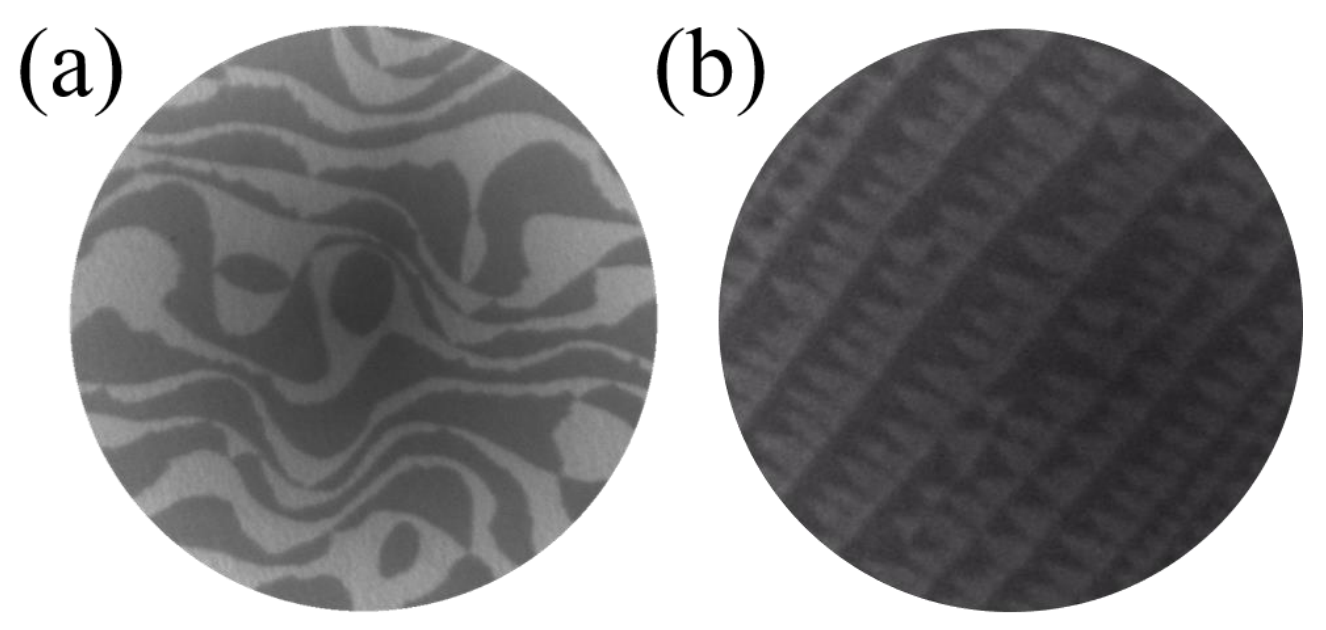

Figure 2. 4 (a) Dark field LEEM image of Si(001). (b) Bright field LEEM image of Si(111).

\subsubsection{Single Crystal Silver Pyramids and Wires}

After obtaining clean and reconstructed $\mathrm{Si}$ substrates, $\mathrm{Ag}$ can be deposited to form plasmonic nanoparticles for PEEM measurements. Ag deposition is performed using an electron beam evaporation source. Silver particles in a crucible is heated to their melting point, causing Ag atoms to evaporate onto the $\mathrm{Si}$ substrates, where they can grow into micron size crystals. The substrate his held at typically around $800 \mathrm{~K}$ temperature, which results in the Stranski-Krastanov (S-K) growth mode. On $\mathrm{Si}(001)$ surface, during the $\mathrm{S}-\mathrm{K}$ growth an $\mathrm{Ag}-(2 \times 3)$ wetting layer forms first (a monolayer of Ag).[63] This enables Ag atoms diffuse across the substrate to form 3D Ag 
cystals with a pyramidal shape. When imaged from the surface normal by LEEM, the Ag pyramids appear to have perfectly flat top plateaus in the $\operatorname{Ag}(001)$ orientation. Depending deposition temperature, the Ag pyramids can either form local structures with lateral dimensions of a few hundreds of nanometers, or form elongated wires with widths of hundreds of nanometers and lengths of tens of microns. Figure 2.5 shows a LEEM image of a single crystal Ag wire, with length of $2.5 \mu \mathrm{m}$ and width of $200 \mathrm{~nm}$.

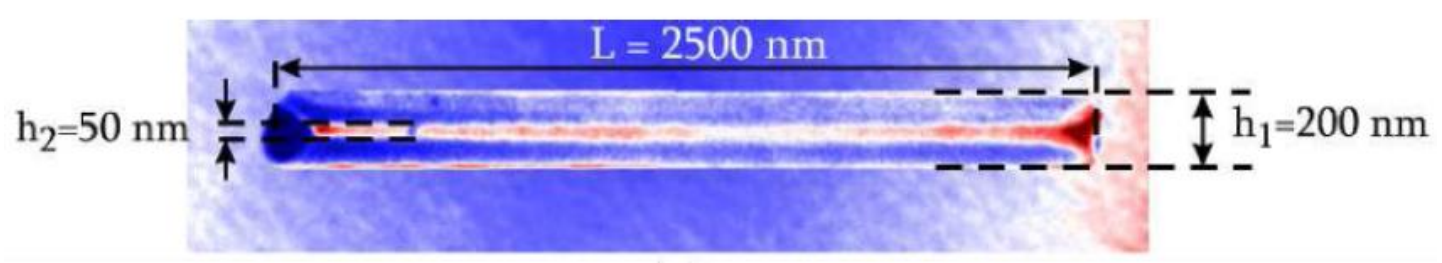

Figure 2. 5 LEEM imge of a Ag wire on $\mathrm{Si}(001)$ substrate.

\subsubsection{Single Crystal Silver Islands}

S-K growth mode also occurs for Ag deposition on $\mathrm{Si}(111)$ substrates, where Ag forms an Ag- $(\sqrt{3} \times \sqrt{3})$ surface lattice wetting layer.[64] After the wetting layer forms, Ag grows in 3D single crystal islands with predominantly triangular shapes in the (111) orientation, or to minor extent, in rectangular shapes with the (001) orientation. The Ag islands heights range from $50 \mathrm{~nm}$ to $300 \mathrm{~nm}$, depending on the substrate temperature during the deposition. A typical LEEM image of a triangular $\mathrm{Ag}$ island is shown in Figure 2.6 (a). The topographic contour shaped contrast represents the atomic terraces of the single crystal $\mathrm{Ag}(111)$ surface. A corresponding LEED pattern is shown in Figure 2.6(b), confirms the (111) surface orientation of the Ag island. 

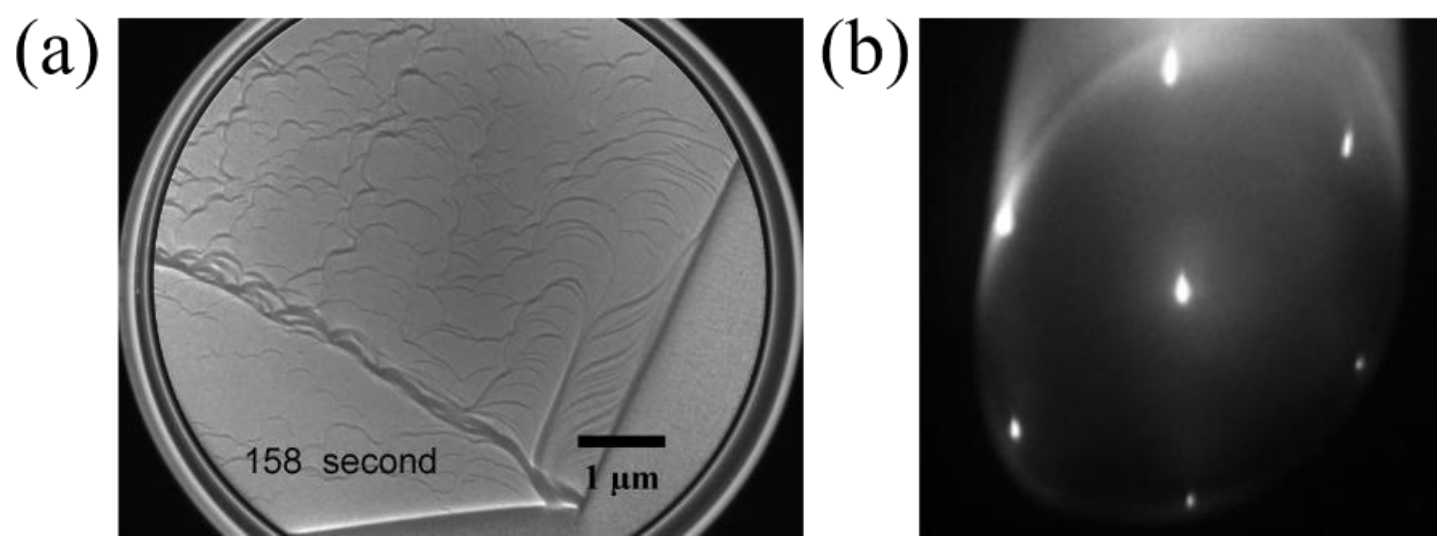

Figure 2. 6 (a) LEEM image of a $\mathrm{Ag}(111)$ island on $\mathrm{Si}(111)$ substrate. (b) a corresponding LEED pattern taken on the Ag island.

\subsubsection{Polycrystal Silver Film}

Polycrystalline Ag film can be prepared by electron beam evaporation (EBE) of Ag on either $\mathrm{Si}(001)$ or $\mathrm{Si}(111)$ substrate, at room temperature. Silicon substrates are processed by Acetone-IPA-DI water procedure to remove dust particles and organic contaminations on the silicon wafer. Prior to Ag evaporation, $2 \mathrm{~nm} \mathrm{Ge}$ film is deposited onto $\mathrm{Si}$ as a transition layer to achieve better smoothness of the Ag film[65]. The deposition can be performed in both Thermionics EBE system or Plassys EBE system (MEB550S). an scanning electron microscopy (SEM) image of $100 \mathrm{~nm}$ polycrystal Ag film is shown in Figure 2.7, with a two slits structures etched by focused ion beam lithography, which will be introduced in the next section. The Ag film demonstrate sufficient flatness regarding to the surface plasmon polaritons propagation. 


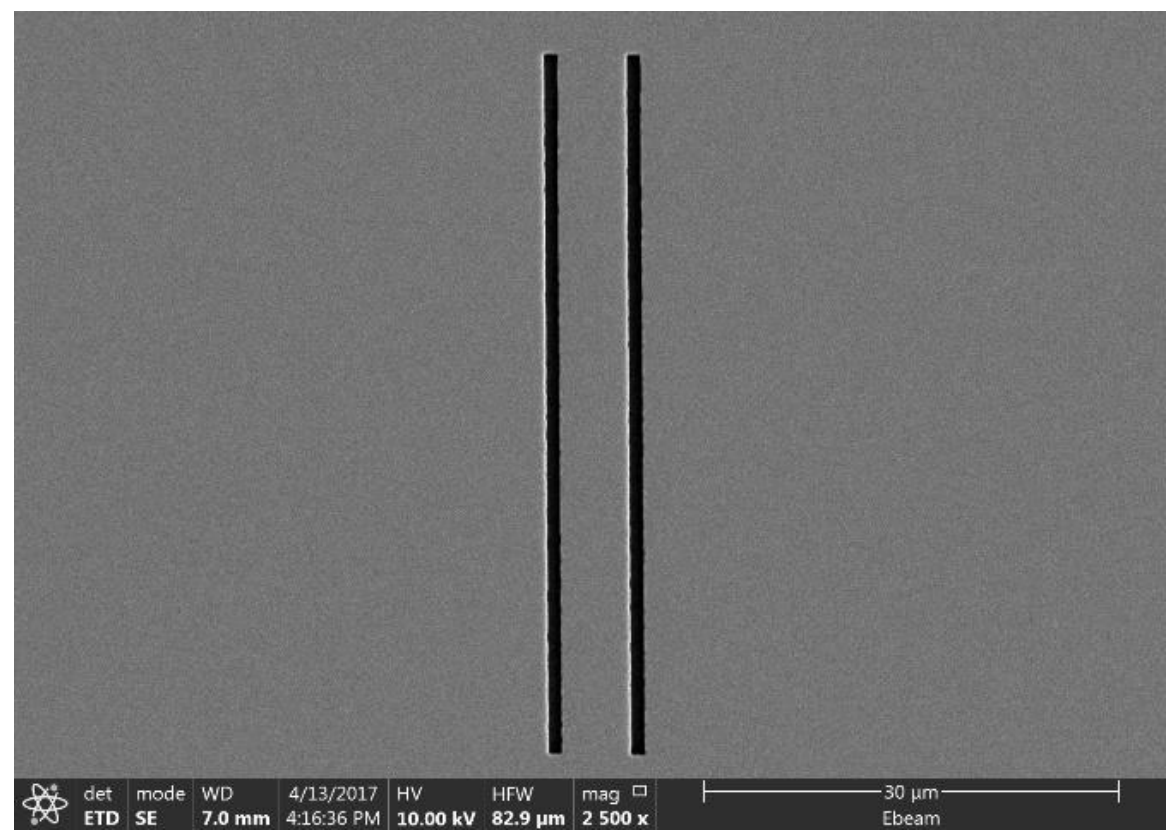

Figure 2. 7 SEM image of polycrystal Ag film with a two slit structures.

\subsubsection{Surface Structuring by Focused Ion Beam}

The coupling structures for surface plasmon polaritons are either naturally formed by the edge of 3D Ag clusters, or etched by focused ion beam (FIB) milling. The FIB milling is performed in a Seiko SMI3050 SE dual beam (FIB-SEM) system. During FIB experiments, Ga+ ions are focused by the ion beam column on to the sample surface, removing Ag from designated patterns. In addition, field emission SEM can also be used to characterize the sample quality, at a specified resolution of $4 \mathrm{~nm}$. A few selected plasmonic coupling structures are shown in Figure 2.8. Figure 2.8 (a) shows a single crystal plasmonic bow tie structure, for study of plasmonic enhancement at 
the bow tie tip. Figure 2.8(b) shows a 6-fold chiral structure (in terms of the height of the lobes), for the study of the chiral plasmonic response. Figure 2.8(c) shows a plasmonic lens coupling, for focusing surface plasmon polariton beams. Figure 2.8(d) shows a plasmonic Archimedean spiral of topological charge $m=8$, for the study of plasmonic vortices.
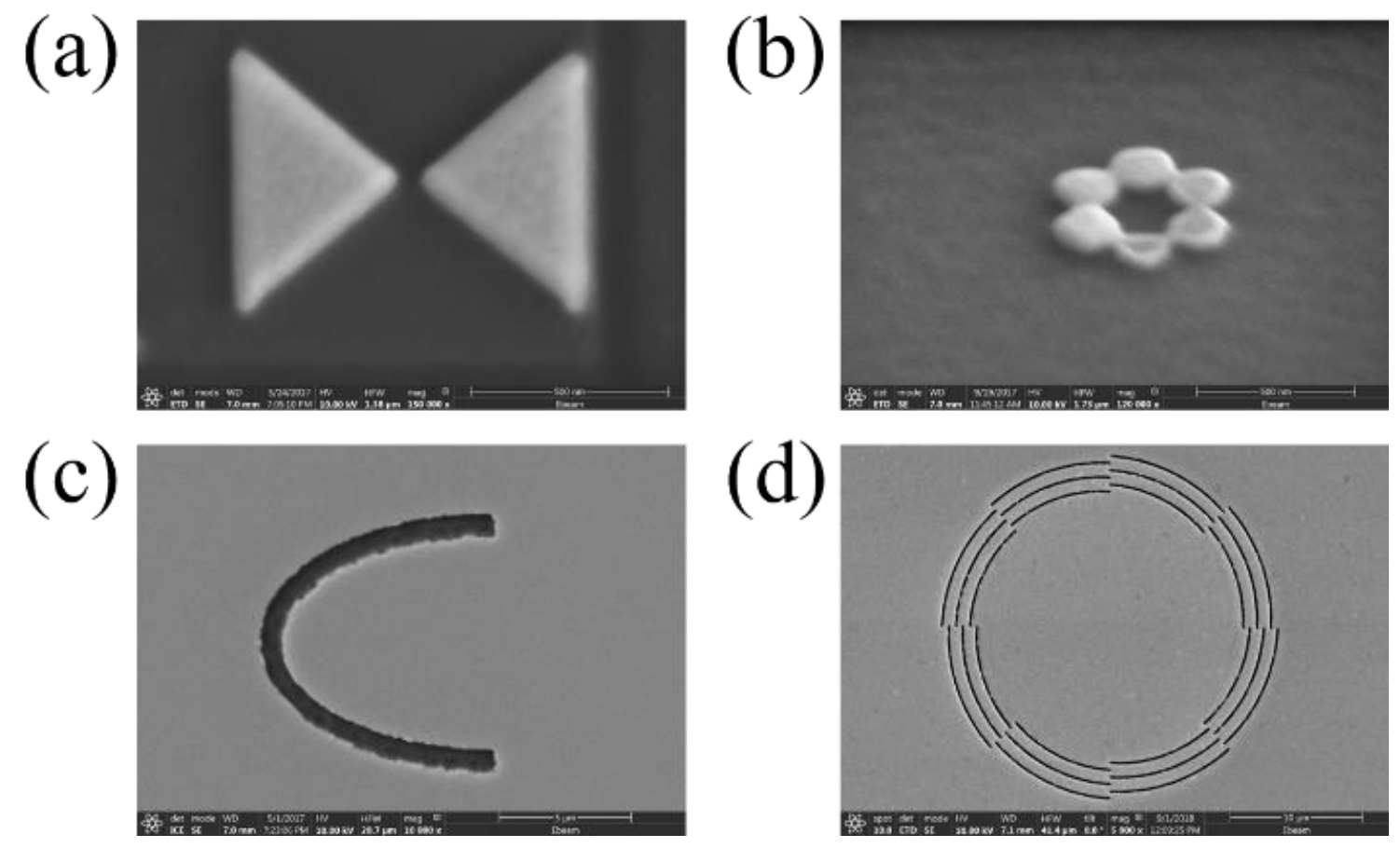

Figure 2. 8 SEM images selected plasmonic coupling structures. (a) nanoscale bowtie structure. (b) chiral plasmonic structure with 6-fold symmetry and varying height. (c) plasmonic lens coupling structure. (d) Archimedean spirals plasmonic vortex generator for topological charge of $\mathbf{m}=8$. 


\subsection{Source of Photoemission}

\subsubsection{Mercury Lamp}

For PEEM experiments, the FE-LEEM P90 is supplied with a $100 \mathrm{~W}$ LOT-Oriel mercury arc lamp. This produces high intensity unpolarized UV light $(\sim 4.9 \mathrm{eV})$, suitable for exciting photoelectrons from most conductors. The most plasmonic material studied so far in our LEEM/PEEM system is silver, which has work function of $\sim 4.6 \mathrm{eV}$ in crystalline form with orientation of (111), and $\sim 4.3 \mathrm{eV}$ in polycrystalline form. Therefore, the UV photon energy is sufficient to induce one-photon photoemission process, which does not contain any plasmonic signal. As a result, UV lamp provides the geometry information of the structures of the plasmonic materials.

The lamp is mounted so that it shines through a viewport onto the sample. The glass in this viewport is a special grade to allow high transmission of UV light. Other viewports on the chamber are made of ordinary glass and are not suitable for use with the UV lamp. The power supply is mounted in the chamber frame, which also monitors the operation time, of the lamp for safety record, because a lamp has a lifetime of 200 300 hours and should be replaced after this time.

\subsubsection{Non-colinear Optical Parametric Amplifier (NOPA)}

The optical system used to generate surface plasmons is shown in Figure 2.9. The fundamental laser source is an Yb-doped fiber laser (Clark-MXR Impulse), which has a center wavelength of $1035 \mathrm{~nm}$ and pulse duration of $250 \mathrm{fs} .[31,66,67]$ The fiber laser typically runs at $1 \mathrm{MHz}$ repetition rate, with energy $1 \mu \mathrm{J}$ per pulse. The output from the Yb-fiber laser is further 
split through a polarization beam splitter (BS). $80 \%$ of its power pumps two $\beta$-BaBO3 (BBO) crystals to produce second $\left(2 \omega_{0}\right)$ and third $\left(3 \omega_{0}\right)$ harmonics of the fundamental beam. The rest $20 \%$ goes into a planar sapphire plate to produce a white light continuum (indicated as white light generation WLG), which further splits into two parts of different wavelength regimes by a bandpass filter. The longer wavelength regime $(>650 \mathrm{~nm})$ of the white light meets the $2 \omega_{0}$ beam at another $\mathrm{BBO}$ crystal, where a portion of the white light, which is the seed light, can be selectively amplified by the $2 \omega_{0}$ pulse, which is the pump light, to produce amplified pulse in the range between $650 \mathrm{~nm}(1.91 \mathrm{eV})$ and $900 \mathrm{~nm}(1.38 \mathrm{eV})$. The wavelength of amplification is dependent on their spatial overlap and relative temporal delay. Such amplification process is referred to as a non-colinear optical parametric amplification (NOPA) process. The criterion for amplification is known as a phase matching condition, where the momenta among the pump, seed and the output light must conserve. The short wavelength regime $(<650 \mathrm{~nm})$ of the white light meets $3 \omega_{0}$ beam to produce amplification in the range of $500 \mathrm{~nm}(2.48 \mathrm{eV})$ to $650 \mathrm{~nm}(1.91 \mathrm{eV})$, according to their phase matching condition. In addition, both amplified pulses can be directed into another optical parametric systems, to generate their second harmonics, which extend the wavelength regime down to around $270 \mathrm{~nm}(4.59 \mathrm{eV})$. Through the whole generated pulse regime, the pulse duration is well compressed to be around 20 fs or less. 


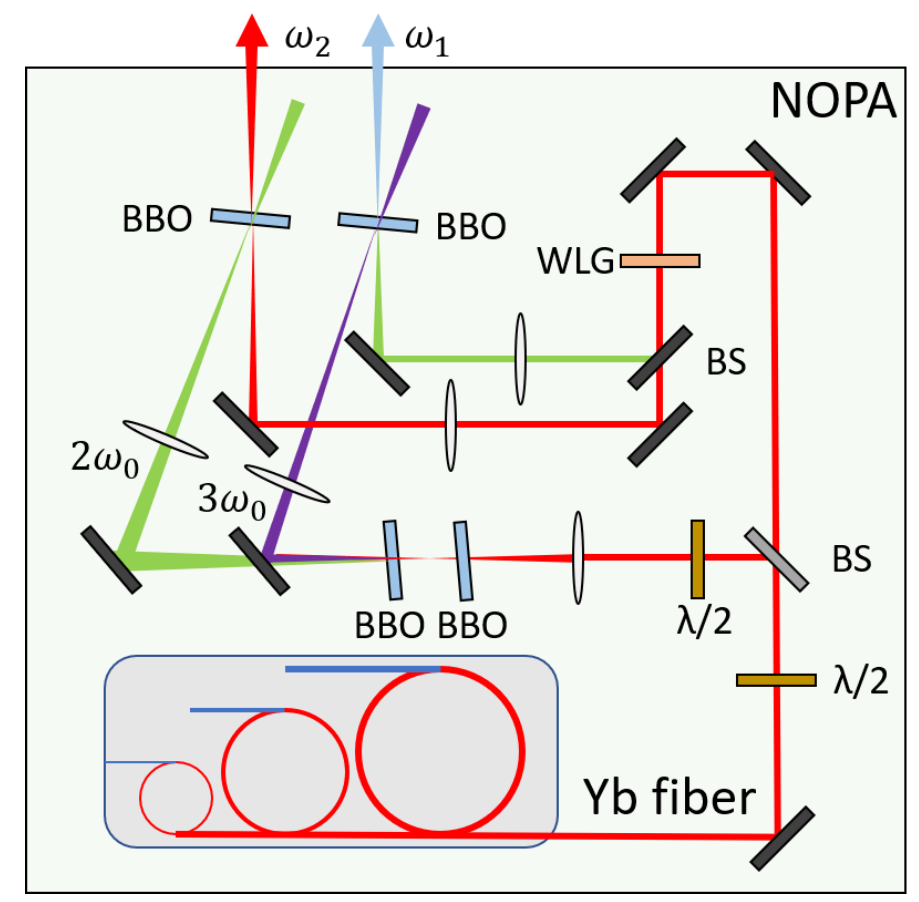

Figure 2.9 Schematic of the NOPA system.

\subsubsection{Interferometric Pump Probe setup}

A crucial part in time resolved pump probe experiment is a Mach-Zehnder interferometer (MZI), which provides two phase locked pump-probe pulse pairs with designated phase delay.[49] A schematic of the MZI is depicted in Figure 2.10. Essentially, an input pulse is split by the first beam splitter into two identical pules, whereby one of the pulses is delayed by a certain amount through a scanning optical stage, which is controlled specifically by a piezoelectric motor. The two pulses meet at the second beam splitter, where the pulse pair is further split into two pulse pairs. One of the output pulse pair is used for experimentation, while the other pulse pair is sent through a monochromator, where a sinusoidal interference pattern is observed dynamically to 
calibrate the time delay of between the pulses. The stability of the piezoelectric motor is on the order of $1 \mathrm{~nm}$, which is sufficient to hold the delay between the pulse pair.

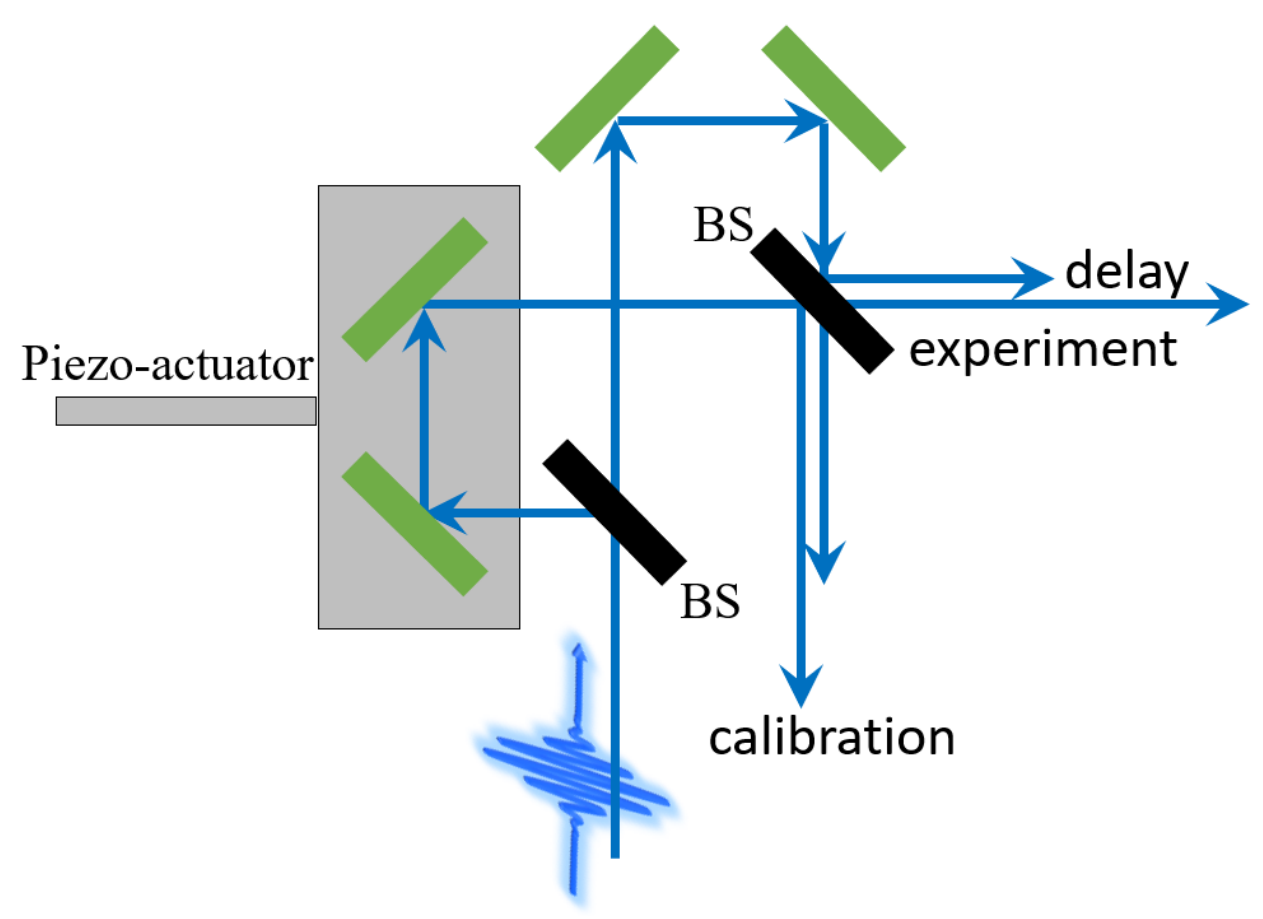

Figure 2. 10 Schematic of Mach Zehnder interferometer. 


\subsection{Simulation Framework}

In this section, I will describe two main methods employed in simulating surface plasmon polariton fields at metal surfaces. The first one is finite difference time domain (FDTD) method [68], which solves Maxwell's equations at each point in space, and use the constitution relation to evolve the electromagnetic fields in time, until a steady state solution is obtained. Such method gives the time-dependent vectorial field evolution of the SPP field, so that we can study the

dynamics of the SPP fields.[30, 69] The second method is based on the Huygens principle, where one assumes the SPP waves are originated from an array of point sources with defined structures, which interfere with each other as they propagate. By superimposing waves from all the point sources, one is able to construct the SPP field distribution by a predefined structure (a set of point sources). By introducing SPP coupling mechanism based on various polarizations of light, I extend the model for the analysis of the vectorial coupling of SPPs. The formulation of both methods is described.

\subsection{Finite Difference Time Domain Algorithm}

Because PEEM experiments contain information of all electromagnetic fields integrated in the optical cycle of excitation, a numerical method that is able to reproduce all field components, describe their coupling, and ultimately simulate a PEEM experiment, is necessary. In this section, I will describe a fully vectorial simulation method, namely finite difference time domain (FDTD) algorithm, which is the basis for simulations of PEEM imaging in this work. The FDTD algorithm 
is based on the curl equations of the electromagnetic fields as an update method, e.g. when there is a change in the electric field, the surrounding magnetic field is updated, and in-turn, the resulting surrounding electric fields. This update process expands the entire problem space, and over designated time window obtains either a steady state solution in continuous wave simulation, , or a time-propagated field solution in pulsed excitation simulations.

In a time domain method, update equations are needed to describe the field evolution. The update equations are all based on time-dependent Maxwell's equations, as shown in equations 3.13.4. The equations describe how a change in the magnetic field at a specific point in space and time, changes the electric field in the surrounding regions, and vice versa.

$$
\begin{gathered}
\nabla \times H(t)=\frac{\partial D(t)}{\partial t} \\
\nabla \times E(t)=-\frac{\partial B(t)}{\partial t} \\
B(t)=\mu(t) H(t) \\
D(t)=\varepsilon(t) E(t)
\end{gathered}
$$

It should be noted that the electromagnetic simulation assumes that PEEM provides a nonlinear measure of electromagnetic fields over time. In other words, I assume that processes where a field decays to generate a hot electron population and hot electrons are subsequently excited by a coincident field, i.e., incoherent mPP processes, are assumed not to contribute to imaging. There is no strong evidence that such processes must be considered to reproduce PEEM images, because they do not contribute to interference phenomena.

In a standard FDTD simulation, a spatially 3D problem space is defined initially, with a size that is determined by the purpose of the simulation. In addition, for all simulations in this thesis, the problem space is discretized in a cartesian Yee grid, with a basis of cubic unit elements, 
where the fundamental unit cell is shown in Figure 3.1. In a typical Yee grid unit cell, the electric and magnetic field components in all three directions must be specified. Positions of the field components are also crucial. At $\mathrm{z}=0, E_{x}$ is placed at $(1,0,0)$ and $E_{y}$ is at $(0,1,0)$. Moreover, because there are adjacent unit cell, the fields $E_{x}$ at $(1,2,0)$ and $E_{y}$ at $(2,1,0)$ must flow continuously into the adjacent unit cells. If the grid terminates at a boundary of the problem space, one can either employ a periodic boundary conditions that insure field continuity, or use zero fields as a hard terminations. Finally, the magnetic field components are located alternatively between the electric field components. By such discretization of space, one can describe the curl equations in Maxwell equations as follows:

$$
\begin{gathered}
\nabla \times\left. H\right|_{t+\frac{\Delta t}{2}}=\varepsilon \frac{\left.E\right|_{t+\Delta t}-\left.E\right|_{t}}{\Delta t} \\
\nabla \times\left. E\right|_{t}=-\mu \frac{\left.H\right|_{t+\frac{\Delta t}{2}}-\left.E\right|_{t-\frac{\Delta t}{2}}}{\Delta t}
\end{gathered}
$$

where $\Delta t$ is the time step of the field evolution. Following the curl equation 3.5-3.6, the four components of electric fields adjacent to $H_{z}$ at $(1,1,0)$, i.e. $E_{x}$ at $(1,0,0),(2,0,0)$ and $E_{y}$ at $(0,1,0)$, $(0,2,0)$, will be used to compute the magnetic field $H_{z}$ at $(1,1,0)$. Similarly, the field of $E_{x}$ and $E_{z}$ will update $H_{y}$, and the field $E_{y}$ and $E_{z}$ will update $H_{x}$. In turn, all the updated magnetic fields will change the electric fields in the next time step. One thing to note is that the $\mathrm{E}$ and $\mathrm{H}$ fields are not updated simultaneously in the simulation, rather the $\mathrm{H}$ field is updated half a time step later than the E field. This is unphysical, because on field is not retarded with respect to the other. However, this sequential update will not affect the physical meaning of the electromagnetic field, because we only consider the EM fields after one cycle of update (both E and $\mathrm{H}$ fields are updated fully).

The plasmonic response to external electric fields of a simulated material is encoded in its dielectric response function $\varepsilon$. For silver, the dielectric function can be represented by the 
experimental evaluation of Johnson \& Christy [1]; the rest of the plasmonic simulation is handled by calculating the field evolution as governed by the Maxwell's equations.

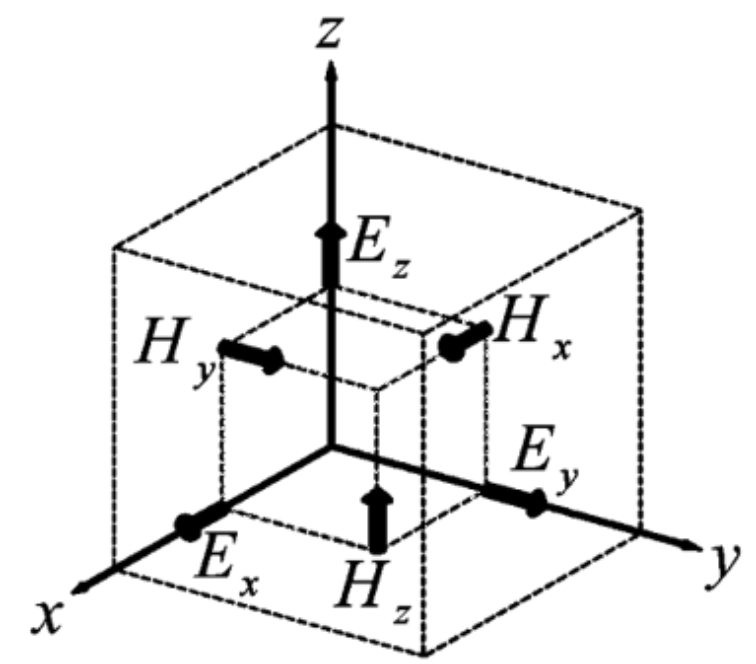

Figure 3. 1 A unit cell of the Yee grid (inner cube) of in a FDTD algorithm. Within one grid, three electric field components and three magnetic field components are marked by arrows. These components are placed at different spatial locations in the cube to update surrounding fields by the curl equations.

Additional constraint on the FDTD method is that the electromagnetic fields must not travel over two grid cells within a single time step, which is insured by the following stability condition:

$$
\Delta t<\frac{1}{c \sqrt{\frac{1}{(\Delta x)^{2}}+\frac{1}{(\Delta y)^{2}}+\frac{1}{(\Delta z)^{2}}}}
$$

where $c$ is the speed of light, $\Delta t$ is the time step, and $\Delta x, \Delta y, \Delta z$ are the spatial grid sizes in the corresponding Cartesian coordinate directions. 


\subsection{Reproduction of Static PEEM Images}

The fully vectorial FDTD simulations output spatio-temporal distributions of the electromagnetic fields. Therefore, for a specific point in the problem space, one obtains the time evolution of all fields, which contribute to the PEEM results. In addition, for non-magnetic materials, and in our case silver, the magnetic part is neglected because of the field strength is two orders-of-magnitude smaller than the electric fields, which dominate the photoemission process. Thus, PEEM mainly images the total local electric field, which is the sum of incident electric and the generated local plasmonic fields.

Following eq. 1.22, the interference pattern imaged by PEEM is computed by integrating the sum of the amplitudes of all electric field components to the $n^{\text {th }}$ power, over one cycle of excitation. In the case of oblique excitation where incidence angle is $70^{\circ}$, the dominating electric field component is the out-of-plane field for p-polarized light. Therefore, the interference pattern between the out-of-plane fields of SPPs and the external light dominates the PEEM contrast, while the in-plane components contribute as a uniform background after time average. In the case of normal incidence PEEM, because light polarization is in the surface plane, therefore the interference pattern is mostly determined by the in-plane fields interference between SPPs and light, while the out-of-plane SPP fields will be averaged out.

Figure 3.2 shows a schematic of the simulation setup of an obliquely excited SPP fields, with a series of $E_{z}$ (out-of-plane) field component as a function of simulation time. The excitation setup of is a 1D SPP wave excited by $p$-polarized (TM wave, the magnetic field is perpendicular to the page) ,30 fs, $400 \mathrm{~nm}$ pulse at a $200 \mathrm{~nm}$ slit located at $-12 \mu \mathrm{m}$ along $\mathrm{Ag} / \mathrm{vacuum}$ interface. The incident light covers the whole surface, and he incidence angle is $70^{\circ}$ (k-vector of light points 
towards the surface, with an $70^{\circ}$ angle from surface normal), and the excitation source is offset by $60 \mathrm{fs}$, i.e. the total field (the sum of the SPP field and the external field) is maximum at $60 \mathrm{fs}$. When the simulation is at $37 \mathrm{fs}$, the excitation wave just arrives to the coupler and launches SPP waves with weak amplitude. At $74 \mathrm{fs}$, the $E_{z}$ field shows an interference pattern with a period of $2.4 \mu \mathrm{m}$. At $112 \mathrm{fs}$, the incident pulse is passed, leaving the pure SPP fields that evolve and propagate along the Ag/vacuum interface. At 149 fs, the pure SPP fields propagate further as well as decay into weaker waves.
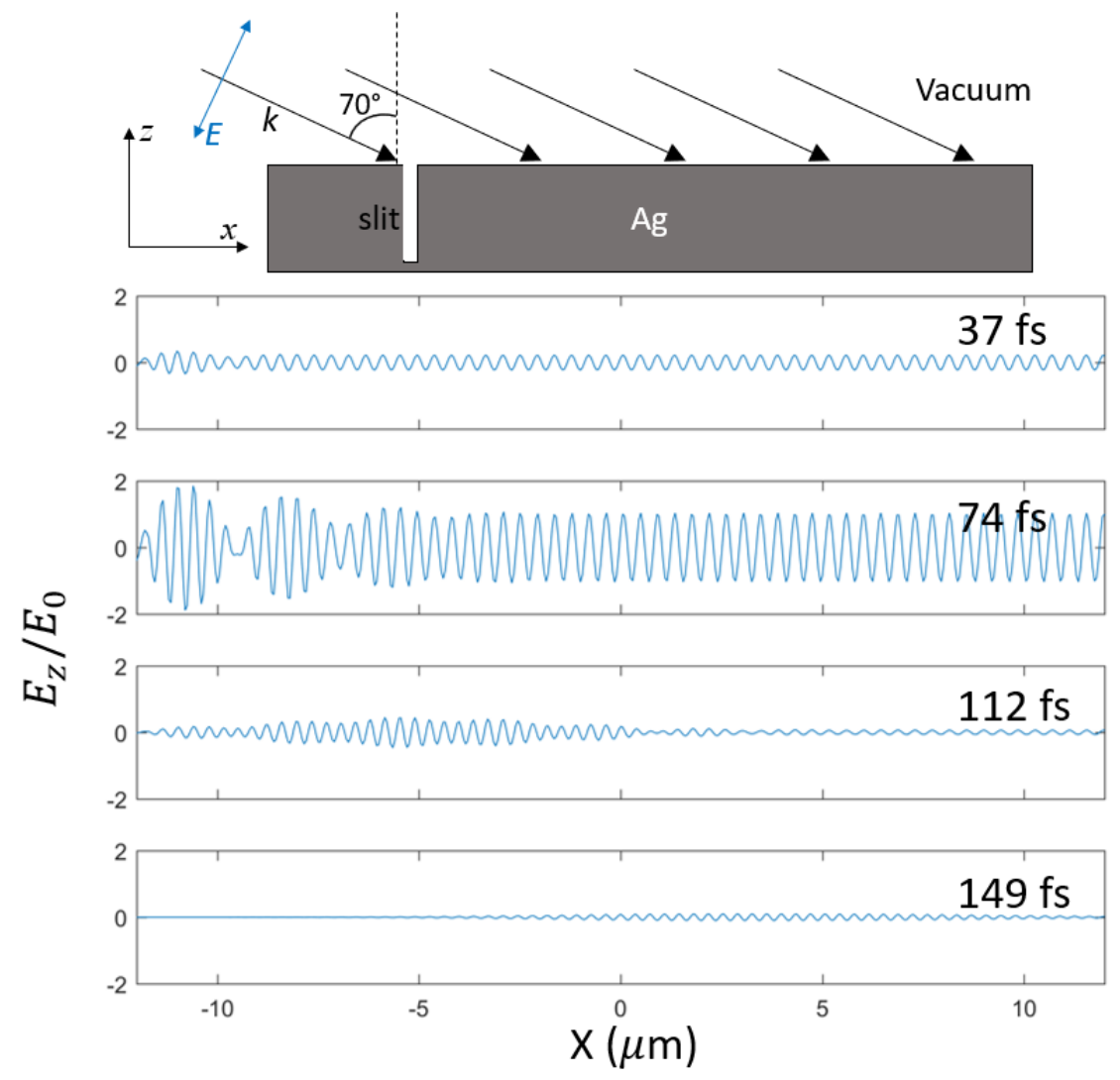

Figure 3. 2 Schematic of the 1-D excitation of SPPs at Ag/vacuum interface, and snapshots of simulated $E_{z}$ field along the Ag/vacuum interface. The SPP wave is launched at $-\mathbf{1 2} \mu \mathrm{m}$, and propagates towards $+x$ direction as the simulation time evolves. Interference with a $\lambda_{\mathrm{B}}$ envelope pattern is seen when the incident pulse is close to, but past its maximum. After the pulse has passed, the pure SPP field propagates and decays. 
Using the calculated fields above, we can exponentiate them to the appropriate power and integrate the result numerically to get the interference pattern observed in PEEM, according to equation 1.22. Figure 3.3 shows the integrated result of the simulated interference pattern representing a $2 \mathrm{PP}$ process, i.e. $n=2$. Starting from $-12 \mu \mathrm{m}$, a distinct periodic pattern is observed, with a period of $2.4 \mu \mathrm{m}$, which is similar to the $E_{z}$ field beating pattern at $74 \mathrm{fs}$ in figure 3.2 Such agreement is due the $E_{z}$ (out-of-plane) component producing much stronger 2PP signal than the in-plane component, thus $E_{z}$ dominates the interference pattern in PEEM.

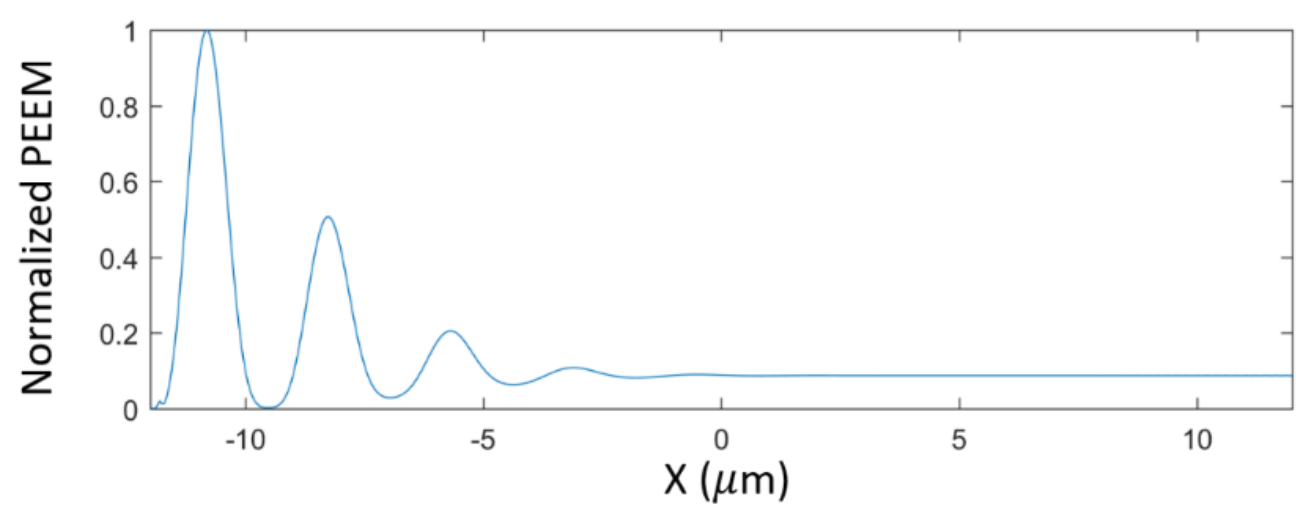

Figure 3. 3 Simulated interference pattern based on the fields in Figure 3.2.2, showing distinct periodic fringes that arise from SPP field beating with the $E_{z}$ component of the excitation field with periodicity $\lambda_{\mathrm{B}}$. 


\subsection{Reproduction of Time-Resolved PEEM}

In the case of a time-resolved PEEM experiment, there are two identical pulses for pump and probe SPP fields. Therefore, the SPP fields excited by the pump light propagates along the sample surfaces, while the probe pulse interferes with the pump excited SPPs with certain interpulse delays. For a specific delay time, the probe pulse arrives at the surface later than the pump pulse, when the pump excited SPP fields have propagated for a distance determined by the delay time. Therefore, the probe pulse can create interference patterns with the pump excited SPP fields, at regions the pump excited SPP fields have reached. By advancing the delay, one is able to acquire a motion of the time-dependent interference pattern, which reflects the evolution of the pumpexcited SPPs.

To provide insight to the time resolved PEEM experiments, a method of computing the time dependent interference pattern is needed. Based on eq. 1.22, the total polarization field is a sum of parts, including the pump and probe fields, each of which has the incident and SPP field components. Thus, a pump-prove delay time $\Delta t$ time dependent field interference pattern can be obtained by the following equation:

$$
I_{P E E M}(x, y, \Delta t) \sim \int_{-\infty}^{t}\left(P_{t o t}\left(x, y, \tau^{\prime}\right)+P_{t o t}\left(x, y, \tau^{\prime}+\Delta t\right)\right)^{2 n} d \tau^{\prime}
$$

where $\boldsymbol{P}_{t o t}$ is the total polarization field and $\Delta t$ represents the pump-probe delay and the signal is integrated over one cycle of pump-probe excitation. If $\Delta t$ is sufficiently long there is no interference between the pump and probe components, and the signal will be just a simple sum of their signals without any dynamical, delay dependent contributions. If the delay $\Delta t$ is reduced, there is a dynamical interaction signal where the pump pulse generates an SPP field wave packet, 
and the external probe field interferes with it. Figure 3.4 shows several calculated time-resolved beating patterns at different time delays that contribute to the calculated PEEM signal in Figure 3.3 .

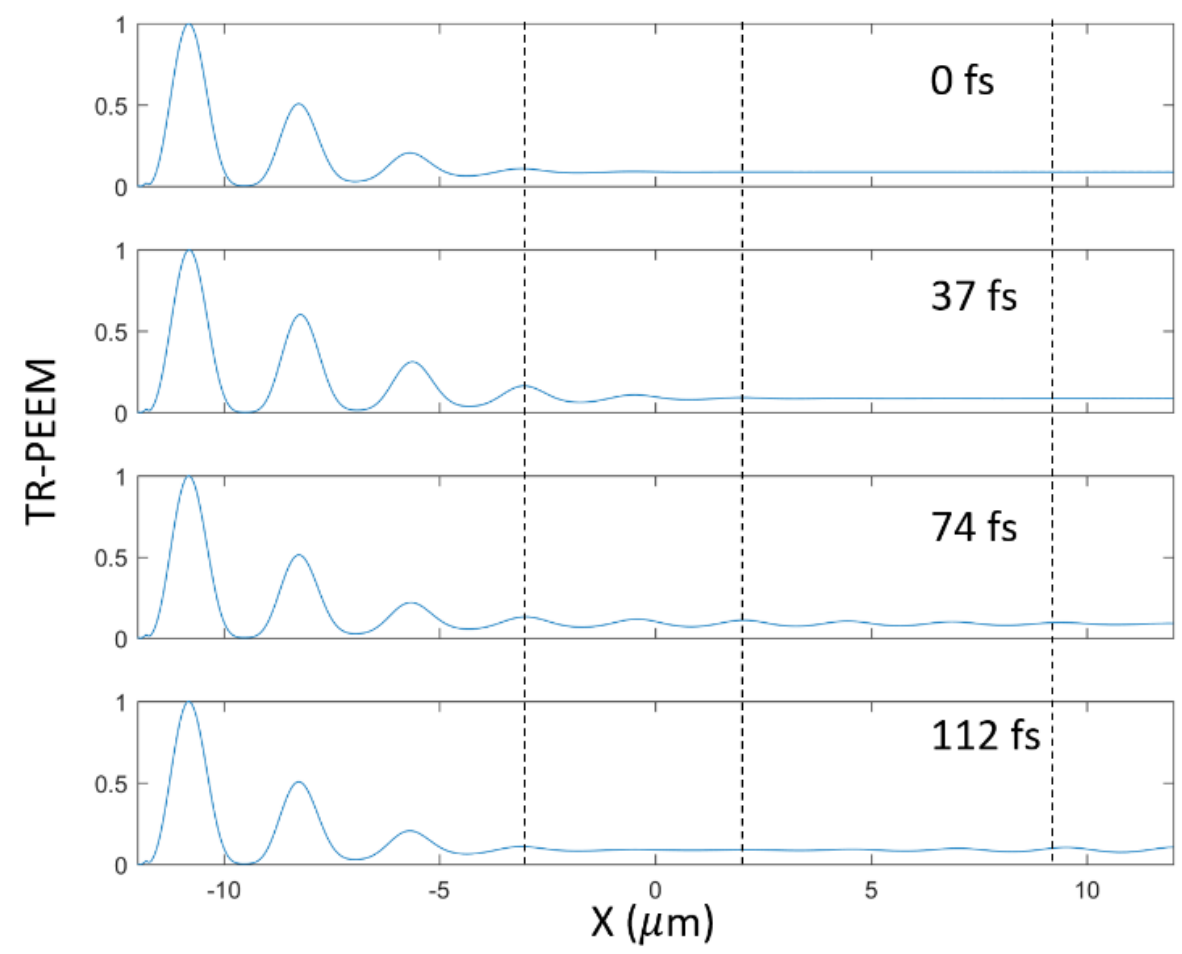

Figure 3. 4 Snapdhots of time-resolved beating patterns, based on the fields in Figure 3.3. At 0 fs, only 4 distinct beats are observed, but at longer $\Delta t$, more beats, due to the $\Delta t$ dependent pump-probe interaction, appear.

To clearly illustrate the dynamic features caused by the SPPs propagation, in Figure 3.5, I construct a 2D map of the 1D interference pattern where the ordinate is the propagation length $x$ and abscissa, the delay time. One can immediately identify four bright, horizonal features at low 
$x$, which correspond to the first four beating fringes; because these fringes are caused by selfinterference, they are less affected by pump-probe delay. In addition, as marked by the dashed line, there is an array of continuously tilted fringes, which correspond to the propagation of beating fringes, or pump-probe interference. Such tilting of the interference maxima in the space-time domain informs on the phase velocity of SPP fields.

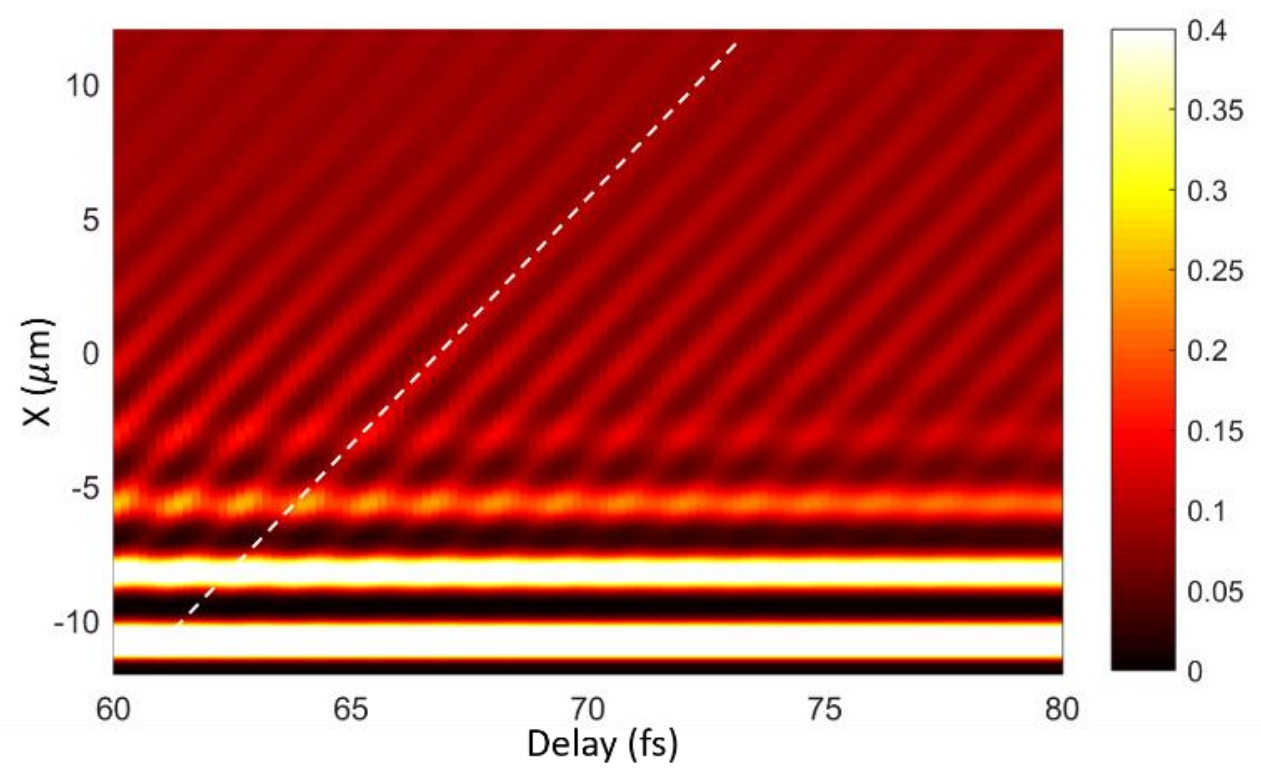

Figure 3.5 2D interferogram of the SPP field discussed in this section. The abscissa represents the pumpprobe delay, and the ordinate the propagation distance. The color scale designates the spatially varying beating amplitude. The dashed line marks the space-time propagation of SPPs beating pattern with the slope giving the SPP phase velocity. 


\subsection{Huygens Principle Algorithm}

In this section, I will describe a polarization dependent Huygens-Fresnel principle calculation[70, 71], where we treat the external light as a superposition of two cross polarized pulses to produce arbitrary polarizations. We first define the Cartesian basis with $p$ - and $s$ polarization base vectors, $E_{p}=E_{0}\left(\begin{array}{l}1 \\ 0\end{array}\right)$ and $E_{s}=E_{0}\left(\begin{array}{l}0 \\ 1\end{array}\right)$. We then construct intermediate polarizations by applying the following matrix transformation (Jones matrix) to the original field, for the half and quarter waveplate (HWP and QWP), respectively.

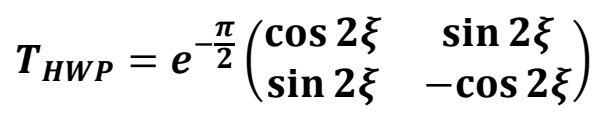

$$
\begin{aligned}
& T_{Q W P}=e^{-\frac{i \pi}{4}}\left(\begin{array}{cc}
\cos ^{2} \xi+i \sin ^{2} \xi & (1-i) \sin \xi \cos \xi \\
(1-i) \sin \xi \cos \xi & \sin ^{2} \xi+i \cos ^{2} \xi
\end{array}\right)
\end{aligned}
$$

where $\xi$ is the waveplate angle and $i=\sqrt{-1}$.

With the knowledge of the amplitude and mutual delay of the orthogonally polarized pulses, which are governed by Jones matrix). We can construct two SPP fields that are associated with each pulse separately. We define $E_{x}$ to be the SPPs component excited by $s$-polarized pulse and $E_{z}$ to be the SPPs component excited by the $p$-polarized pulse. Therefore, their amplitude and phase will follow the same matrix transformation the same way as the excitation light. Consequently, we can superimpose the fields to produce the SPP fields excited by arbitrary polarized light, as follows,

$$
E(t)=E_{x} e^{\left(-\frac{t^{2}}{\Delta^{2}}\right)} \sin (\omega t) \widehat{x}+E_{z} e^{\left(-\frac{(t-\delta t)^{2}}{\Delta^{2}}\right)} \sin (\omega(t-\delta t)) \hat{z}
$$

where $\Delta$ is the FWHM of the Gaussian pulse and $\delta t$ is the delay between cross polarized pulses in $x$ and $z$ directions. In the simplest coupling case, a single slit coupling structure, we model the 
whole slit as a series of points sources defined by unit vectors $\widehat{\boldsymbol{n}}_{\|}(\boldsymbol{r})$ and $\widehat{\boldsymbol{n}}_{\perp}(\boldsymbol{r})$, which are vectors perpendicular to the slit in the surface plane, and along surface normal respectively, as shown in Figure 3.6. These two vectors will determine the excitation pulse into plasmon point sources based on the product $\boldsymbol{E} \cdot \widehat{\boldsymbol{n}}_{\|}$and $\boldsymbol{E} \cdot \widehat{\boldsymbol{n}}_{\perp}$. For more complicated structures, we will model it as a set of slits with proper orientations. After defining the structures, the total plasmon response is then quantified by an integration over a time as follows,

$$
P_{s p p}(t)=\int_{-\infty}^{t} C_{e f f}\left(1-e^{-\gamma_{d}(t-\tau)}\right)\left(E(\tau) \cdot \widehat{n}_{\|}+E(\tau) \cdot \widehat{n}_{\perp}\right) d \tau
$$

where the first bracket is the time domain response function from the Drude model of the dielectric function, and the second one shows the superposition of the generated SPP fields from the unit vectors that define a coupling structure.

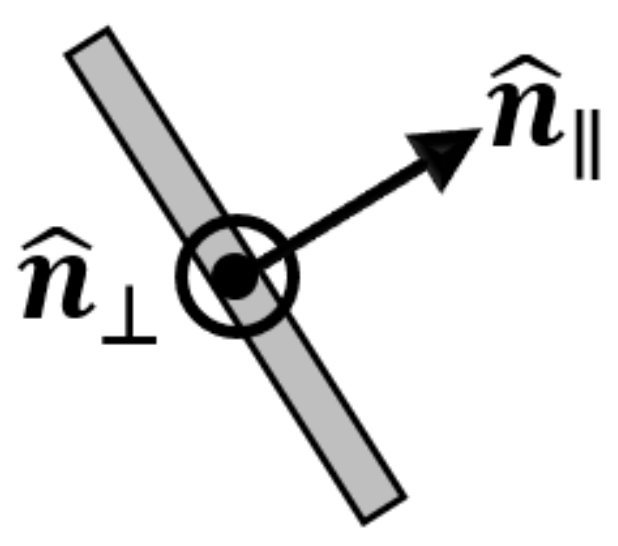

Figure 3. 6 Schematic of the vectorial coupling on a simple slit structure.

The dispersive and dissipative evolution of the SPP field in real space is calculated as follows, First, the SPP wave packet described in time domain is Fourier transformed to frequency space, where one is able to separate all frequency components of the SPP waves. Then, for each 
frequency component (or $k$-component), its phase and damping amplitude is simulated according to the complex wave vector, which is determined by the frequency dependent dielectric function of metals. From the calculated $k$-space propagation, all the frequency components are Fourier transformed back to the real space to obtain the spatial maps of the fields. The PEEM image is finally calculated by integrating the total polarization field raised by the 4th power for the duration of the experiment

$$
I_{P E E M}(x, y)=\int_{-\infty}^{+\infty}\left|E_{e x}\left(x, y, t^{\prime}\right)+P_{s p p}\left(x, y, t^{\prime}\right)\right|^{4} \mathrm{~d} t^{\prime}
$$

To verify the analytical model, we apply the scheme to a simple point source. Under left circularly polarized light (LCP), by rotating the vector $\widehat{\boldsymbol{n}}_{\|}$, which is same as rotating the direction of a sub-wavelength slit for plasmon coupling, we measured the excited SPPs phase $1 \mu \mathrm{m}$ away from the source. The results are shown in Figure 3.7(a), where the SPP phase varies linearly by a total of $\pi$, as expected.[72] Another test simulation is to acquire the PEEM yield while tuning the input polarization as would a QWP; this is plotted in Figure 3.7(b). The PEEM yield is symmetric with respect to the zero point (p-polarization) and has two minima at LCP and right circularly polarized light (RCP). 

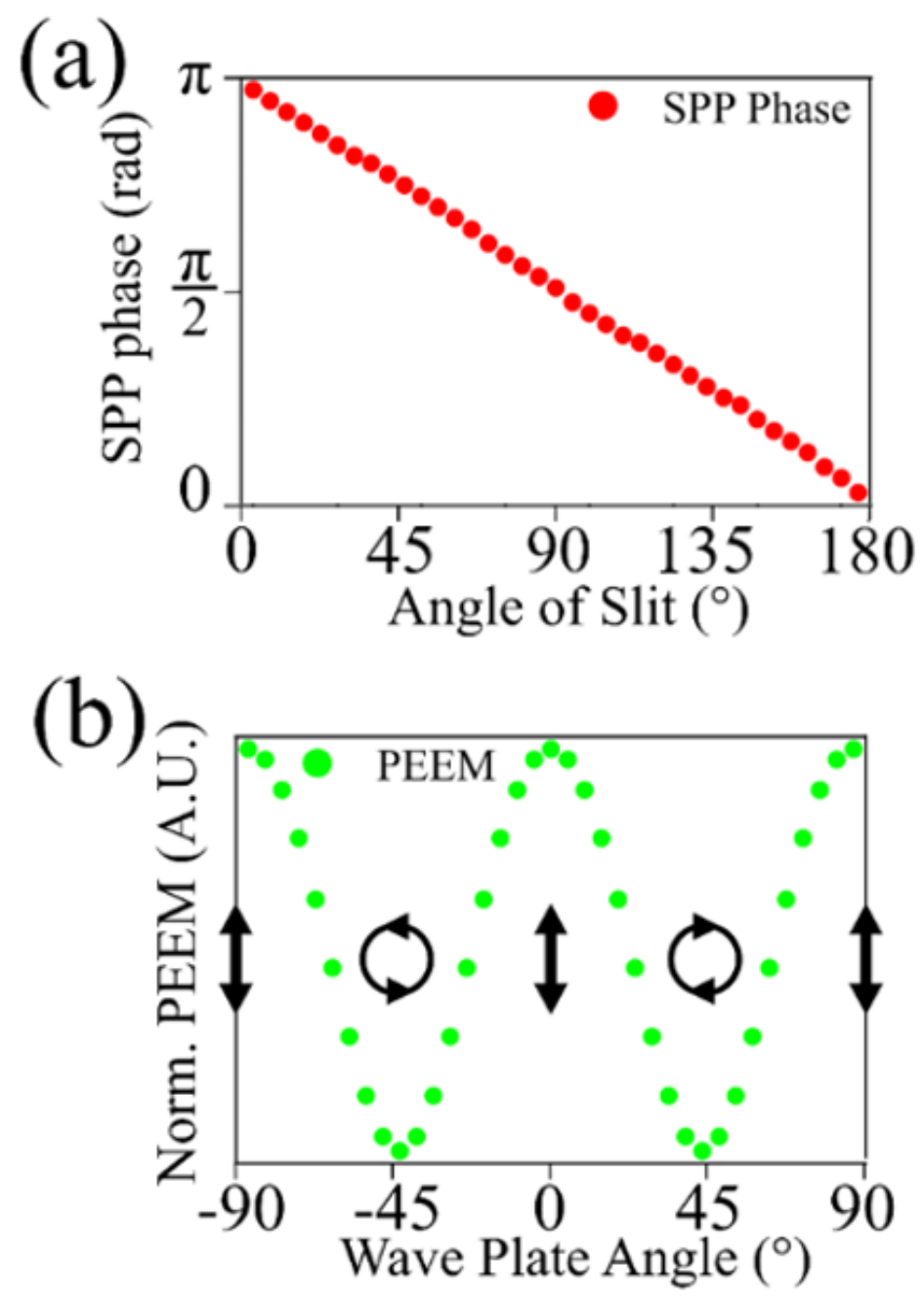

Figure 3. 7 (a) Simulated SPP phase upon changing the unit vector continuously with LCP excitation. (b) Photoemission yield from a single point source as a function of quarter wave plate angle, which defines the light elipticity. 


\subsection{Propagating Surface Plasmon Polaritons (SPPs)}

The simplest excitation geometry of SPP waves is based on illuminating a quasi-1D nanostructures, such as single slits or nanowires. In this chapter, I will describe the field distribution of SPPs launched from quasi-1D Ag nanowires grown on Si(100) substrate.[37, 38, 73] In addition, I will go beyond the 1D coupling structure, and discuss how SPPs are launched from 2D Ag islands grown on $\mathrm{Si}(111)$ substrate, by imaging its 2D field SPPs interference pattern distribution.[30, 31, 35] The 2D fields are more complicated than the $1 \mathrm{D}$ case, which is due to the interference of multiple quasi-1D SPP waves coupled or reflected at edges of an Ag island. Finally, I will describe the dynamics of SPPs propagating on a micron scale Ag island and its corresponding simulations.

\subsection{Propagating SPPs on Ag Wire}

\subsubsection{Co-propagation between Light and SPPs}

The spatial imaging and time-resolved dynamics of SPPs on Ag single-crystal nanowire structures on $\mathrm{Si}$ substrates are the main topics of this section. On the $\mathrm{Si}(100)$ surface, the Ag crystals have a (100) orientation and form either rectangular pyramids or pyramidal nanowires. The pyramids have typical lateral dimensions of $200 \times 100 \mathrm{~nm}$ and $\sim 100 \mathrm{~nm}$ height. In the case of nanowires, one of the lateral dimensions can extend to $10 \mu \mathrm{m}$ or more along one of the two highsymmetry directions of the substrate. 
I first describe the SPP modes imaged for a $20 \mu \mathrm{m}$ long single crystal Ag nanowire. The Ag nanowires are grown epitaxially on clean $\operatorname{Si}(100)$ substrate, which is cleaned by flashing to $1200 \mathrm{~K}$ prior to silver vapor deposition. The lateral dimensions of the silver nanowire are measured by 1 PP-PEEM, which is acquired by one-photon photoemission with an Hg lamp excitation, in Figure 4.1 (a). The excitation light for 2PP is p-polarized, so that its electric field oscillates in the optical plane. The in-plane k-vector direction of the incident light is marked by the red arrow in Figure 4.1 (e). The excitation light illuminates the entire field-of-view of PEEM microscope.

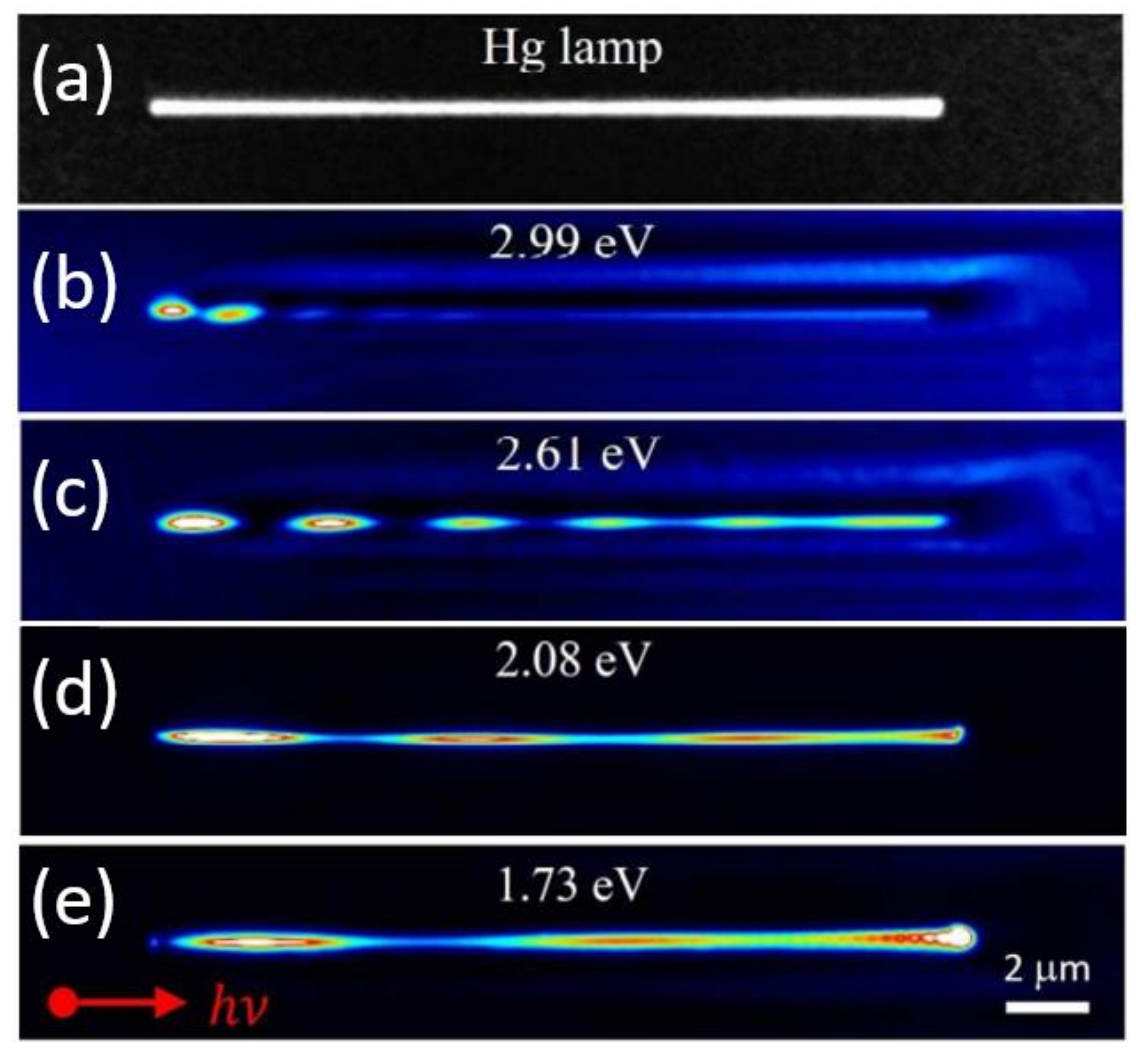

Figure 4. 1 (a) Experimental 1PPE-PEEM image of the lateral dimensions a $20 \mu \mathrm{m}$ long Ag wire acquired with an Hg lamp $(\mathrm{hv}=4.89 \mathrm{eV})$. ( $\mathrm{b}-\mathrm{f})$ Wavelength-dependent 2PP-PEEM measurements of SPPs on the same wire as in (a). The red arrow indicates the propagation direction of the excitation light. Light entering at $70^{\circ}$ from the surface normal from the left, dominantly excites SPPs from the left edge. The excitation photon energies are indicated in $(b-e)$. 
In the case of the oblique $p$-polarized excitation (electric field oscillates in the optical plane, light k-vector parallel to the long axis) of Ag wires, as indicated in (e), light excites the SPP modes at both ends of the wire (mostly visible in (e)). The excited SPPs then propagate toward the center at the local speed of light. Because of the mismatch between the in-plane k-vectors of the external fields and the SPP fields [Figure 1.4], the SPP wavelength is always shorter than the wavelength of the excitation light. Therefore, the superposition of the external light fields and the surface plasmon polariton fields is modulated to form stationary interference patterns with a period of $\lambda_{B}$, which is imaged by the PEEM. The beating patterns recorded by the PEEM can be represented by the equation 1.23. The much weaker modulation orthogonal to the wire long axis in the surrounding region in Figure 4.1 (b)(c) is created by the interference between the incident light and scattered light field from the Ag wire, which is not of plasmonic origin.

The observed SPP beating patterns for selected excitation wavelengths are shown in Figure 4.1 (b)-(e). By tuning the wavelength of the NOPA in the UV-vis-IR region, a clear increase in the beating period is observed associated with a longer excitation wavelength, which is due to the smaller mismatch between their momenta. At longer wavelengths SPPs become more light like, and their k-vector approaches that of light in vacuum. This is evident in Figure 1.4, where the gap between the SPPs dispersion and the light line reduces with smaller photon energy (longer light wavelength).

Figure 4.2 plots the observed values of $\lambda_{B}$ from the measurements and the calculated values from equation 1.23 as a function of the excitation wavelength and angle $\gamma$, where $\gamma$ is the angle between the k-vectors of SPPs and the in-plane external light. The calculated SPPs beating periods are based on the interference between in-plane light wavelength and the SPP wavelength, which is obtained from equation 1.16 based on a Drude model for the dielectric function of the 
$\mathrm{Ag} / \mathrm{vacuum}$ interface. With UV excitation ( $\mathrm{h} v=2.99 \mathrm{eV}$; Figure $4.1(\mathrm{~b})$ ), the SPP interference pattern decays within several $\mu \mathrm{m}$ from where it is coupled at the left side of the image, because of the strong damping of SPPs. By contrast, in the vis-IR region, the wave packets are more weakly confined to Ag and thus propagate over the entire $20 \mu \mathrm{m}$ length without significant damping, as shown Figure 4.1 (c)-(e). At the longest wavelengths, some of the SPP field reflects back from the right edge, causing a more complicated interference pattern when the reflected SPP has appreciable amplitude.

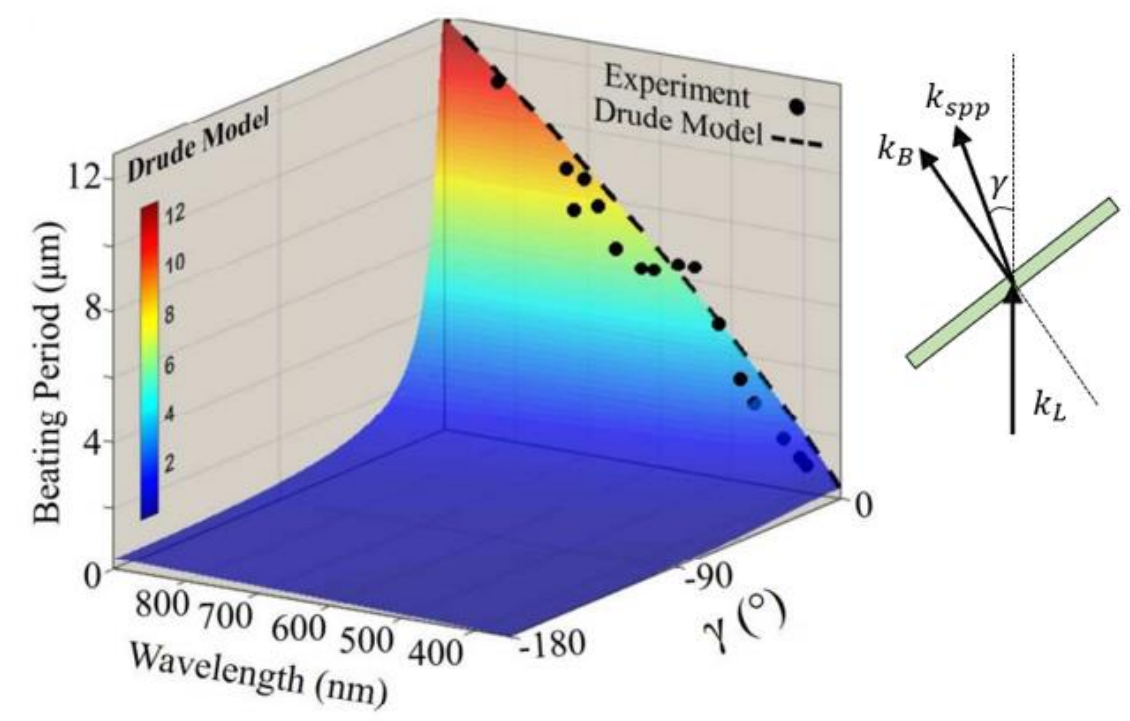

Figure 4. 2 Calculated and measured (dots) beating period $\lambda_{B}$ (color scale) for the incident excitation at $\theta=$ $70^{\circ}$ as a function of excitation wavelength and the angle $\gamma$ between the propagation k-vectors of the in-plane component of the external and the SPP fields. The schematic on the right shows the vectors of an example coupling angle where $\gamma$ is nonzero. 
In the near-IR excitation region $(\mathrm{h} v=1.73 \mathrm{eV} ; 4.1(\mathrm{e}))$, an additional modulation with a much shorter period $(346 \mathrm{~nm})$ appears at the far end of the wire. Such modulation is also caused by SPPs, but in this case, they propagate from the far end toward the near-end; consequently, the propagation k-vector has the same magnitude as the forward SPP wave packet but in the opposite direction $\left(\gamma=180^{\circ}\right)$. Such short period interference is predicted in Figure 4.2, and the amplitude decays faster than that for the copropagating fields because the counter-propagating fields overlap for a shorter span of space and time.

Therefore, by imaging the wavelength-dependent beating patterns of the forward propagating SPP waves on the quasi-1D metal nanowires, we can extract the pure SPP wavelength (momentum) according to Eq. 1.23, thus map out the complex SPP dispersion function at the Ag nanowire/vacuum interface as a function of the excitation photon energy.

\subsubsection{Orthogonal Propagation between Light and SPPs}

In addition to the co-propagating SPPs along the wire long axis, propagating SPPs mode can be launched when the wire long axis is perpendicular to the $k$-vector of light. In Fig. 4.3, I show a PEEM image of such excitation scheme; a $2.5 \mu \mathrm{m} \mathrm{Ag}$ wire is horizontally orientated in the image, and the excitation light $(3.3 \mathrm{eV})$ comes at an oblique angle $\left(70^{\circ}\right.$ from the surface normal) with its in-plane $k$-vector perpendicular with respect to the wire long axis, and indicated by the white arrow. When it is illuminated by $p$-polarized light (the in-plane electric fields polarization is marked at the wire ends), SPPs are launched from both wire ends and propagate toward the wire center, as indicated by the schematic SPP wave in Fig. 4.3. Combining the external field, PEEM images the SPPs beating pattern, in which case the angle $\gamma$ is $90^{\circ}$. Therefore, the period of beating is now $\sim 340 \mathrm{~nm}$, which significantly reduced according to Eq. 1.23 and Fig. 4.2. One thing to note 
is that the photoemission from the wire is symmetric with respect to the center of the wire; this is because the excitation polarization is mirror symmetric, the same as the Ag wire. The periodic beating pattern should be considered as a SPP standing wave rather than being caused by interference with the excitation light, because the two fields are orthogonal in this geometry.

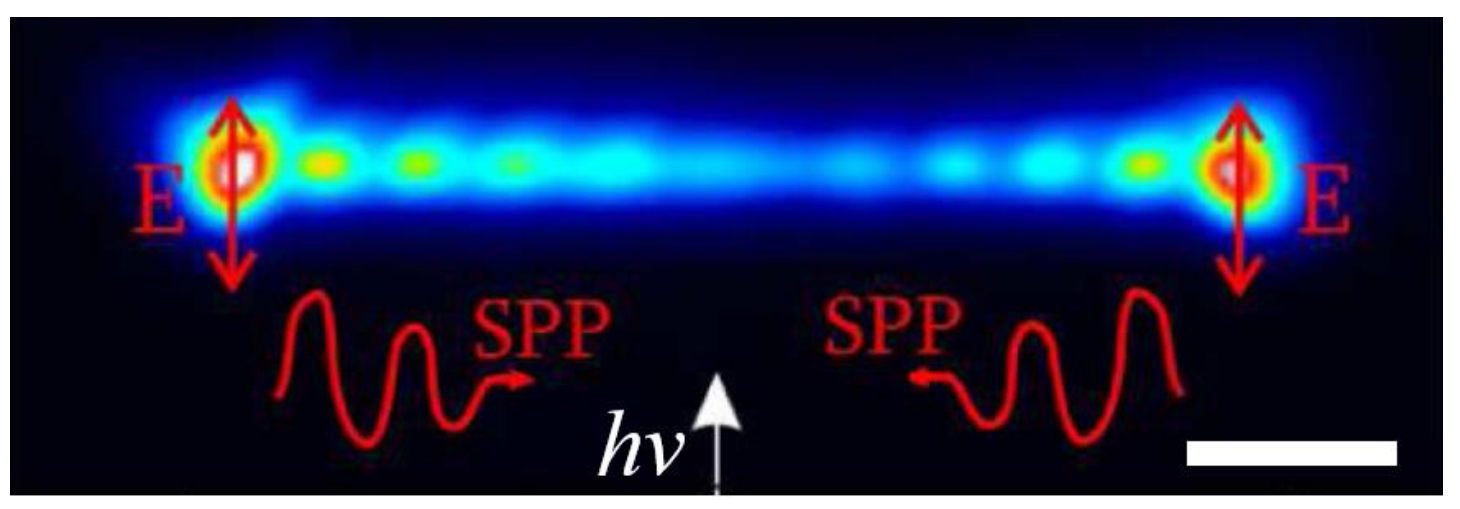

Figure 4. 3 PEEM image of a $2.5 \mu \mathrm{m}$ Ag wire excited by $3.3 \mathrm{eV}$-polarized light, when the k-vector of light orthogonal to the wire long axis. Scale bar is $500 \mathrm{~nm}$.

The corresponding finite difference time domain (FDTD) simulation at the vertical cross section of the Ag wire can help understand more about the SPP field distribution. In Fig. 4.4(a), a schematic of the FDTD simulation is plotted, and a vertical dashed line marks the region we take for field integration to get PEEM images. Fig. 4.4(b) shows a time-averaged field intensity across the Ag wire, excited by p-polarized light at $3.3 \mathrm{eV}$, where one can see clear modulation at the top of the wire, which corresponds to the PEEM measurement if Fig 4.3. Additionally, because the simulated wire has a height of $70 \mathrm{~nm}$, SPP fields at $3.3 \mathrm{eV}$ does not penetrate deep into the $\mathrm{Ag}$ 
wire bulk. Finally, one could observe that fields at the Si bulk is much weaker than at the Ag surface, because the high refractive index of the Si.

(a)
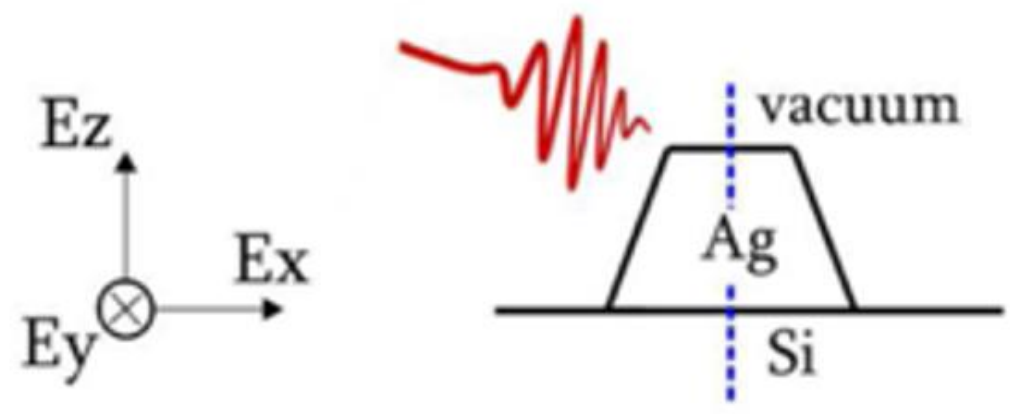

(b)

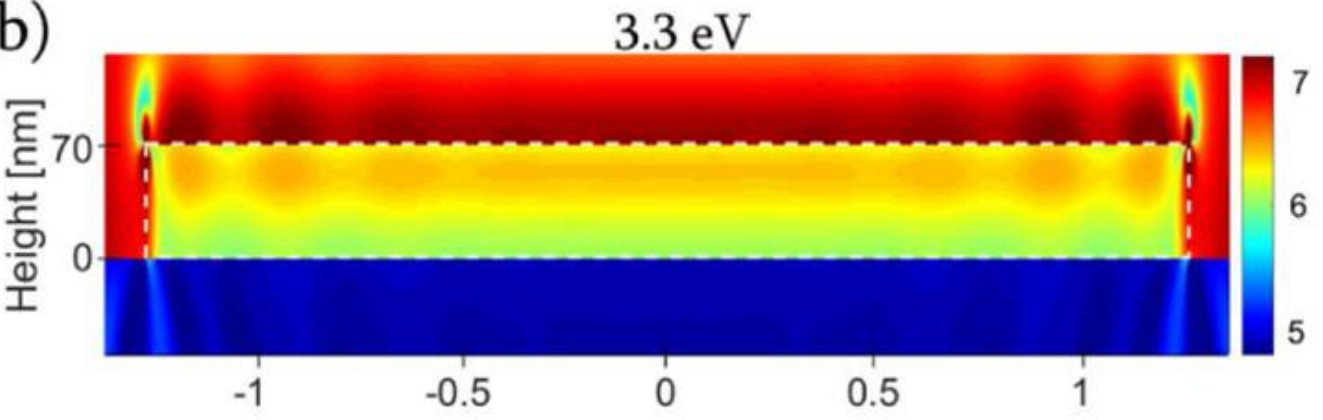

Figure 4. 4 (a) schematic of the simulation setup for wire orthogonal to the k-vector of the excitation light. (b) Time-averaged field distribution at the cross section marked in (a).

Similar to the dispersion mapping in Fig. 4.2, a dispersion relation can be mapped out in the case of orthogonal excitations, which is shown in Fig. 4.5. One can see that the measured dispersion relation follows the trend of the calculated dispersion based on the equation 1.16 and Drude model for Ag dielectric function, but with measured SPP wavelength consistently shifted to a shorter range for the photon energies used. Such effect can be attributed to the geometry of the wire, which the SPP field at the top, compared with the SPP fields at a flat Ag surface. 


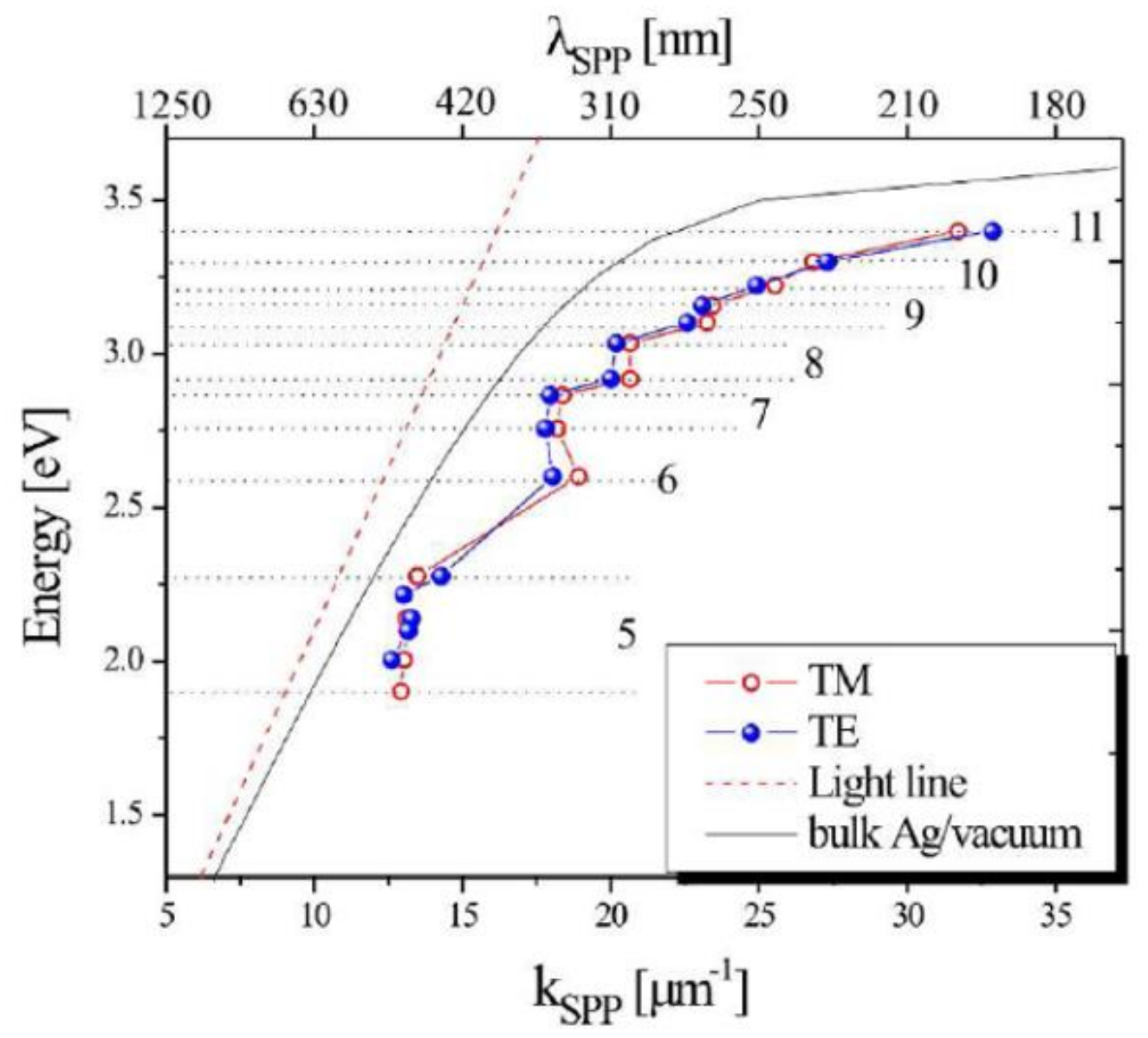

Figure 4. 5 SPPs dispersion relation of the orthogonally placed Ag wire.

When the photon energy is lower than $\sim 1.9 \mathrm{eV}$, a new mode appears at the $\mathrm{Ag} / \mathrm{Si}$ interface, which has much stronger field enhancement compared to the $\mathrm{Ag} /$ Vacuum interface. Fig 4.6 plots PEEM images at selected photon energies below $1.9 \mathrm{eV}$, along with their line profiles. Unlike the previous excitation at higher photon energy, the PEEM images now have a split distribution along the wire long axis, marked by the two white dashed rectangles on the top and bottom. These two modes are attributed to the $\mathrm{Ag} / \mathrm{Si}$ interface SPPs, which have significantly shorter period due to the high index of refraction of Si surface and thus a lower resonance energy.[4] As the photon 
energy is tuned, the top and bottom modes seem to light up at different excitation energies, which means they have different resonance energies. Because the Ag wire has a pyramidal shape, the top and bottom edges do not have equal length, thus it is not unexpected to have different resonance energies.
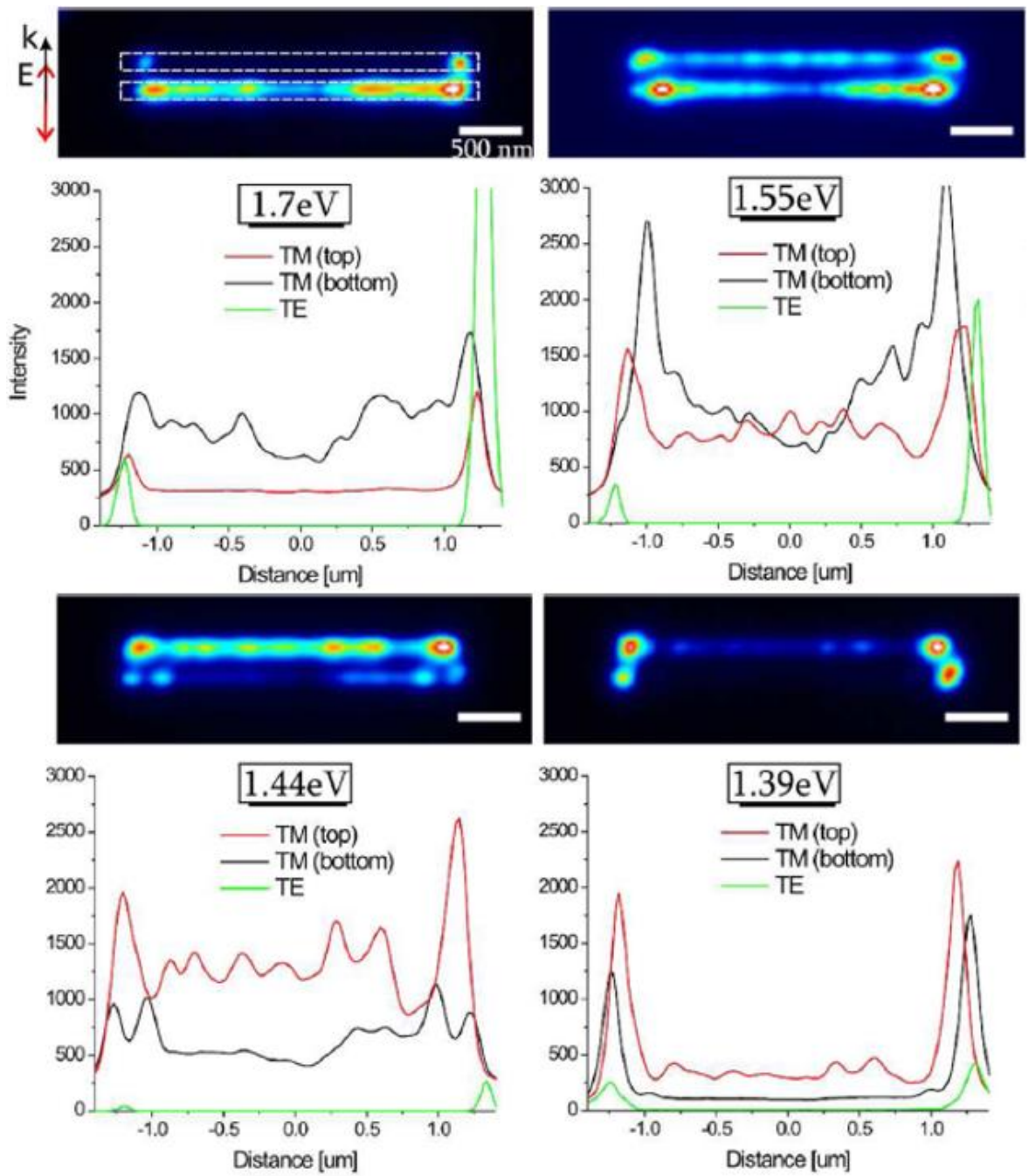

Figure 4. $6 \mathrm{Ag} / \mathrm{Si}$ interface plasmon modes at selected photon energies. 


\subsection{Propagating SPPs on Ag Islands}

\subsubsection{The Vectorial Nature of SPPs Coupling}

While PEEM imaging reveals a simple case of wave interference in the quasi-1D case, more complicated plasmonic phenomena can be examined by imaging plasmon excitations in two dimensions, where the vectorial nature of the coupling of light into SPP modes is revealed. Figure 4.7 shows the experimental PEEM image of a truncated equilateral single crystal Ag island excited by p-polarized pulses, with photon energy $h v=3.21 \mathrm{eV}$. The light comes from the bottom of the island, at an angle of $70^{\circ}$ from surface normal. The in-plane k-vector is marked by the red arrow. The image shows a complicated interference pattern, which, as in the case of 1D structures, represents interferences of the external fields with the SPP modes excited at each crystal edge, as well as their reflections from the crystal edges. The bottom edge is the major SPP launcher, which contributes to the 4 strongest interference beatings with period of $\sim 1.8 \mu \mathrm{m}$ along the k-vector of light. This should agree with the interference in the quasi-1D wire because of its quasi-1D nature. In addition, fringes with short beating period are observed on the far side of the island, which is due to the interference of the back propagating SPPs coupled from the far side edge and the forward propagating light, and the interference between the reflected SPPs and the external light. Lastly, there are two dark regions with weak photoemission, on the left and right side of the island. This is due to the lack of SPP coupling based on the angle of the island edge and the excitation wavelength. 


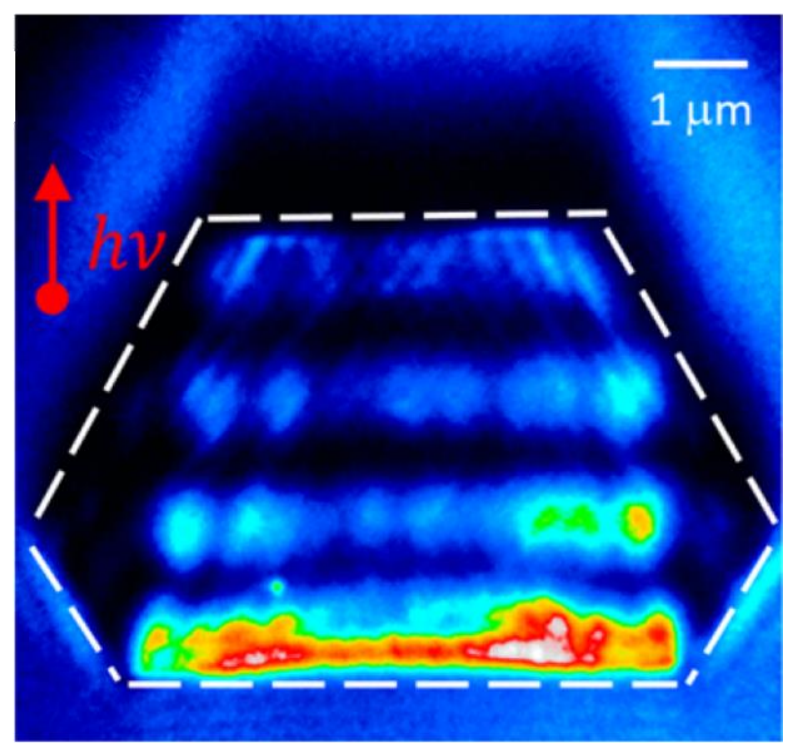

Figure 4. 7 Experimental PEEM image of the SPPs on a truncated triangular Ag(111) island, excited with $p$ polarized $h v=3.21 \mathrm{eV}$ light. Red arrow indicates the direction of the k-vector of the incident light. White dashed lines demark the island edges.

To reveal the mechanism behind the formation of the interference patterns, 3D-FDTD simulation with the same crystal structure and excitation geometry is performed using the selfmade FDTD simulation package. The simulated PEEM image is calculated by integration of the total polarization field over one excitation pulse, as already described in eq 1.22. In Figure 4.8, the simulated PEEM image reproduces the dominant features of the experimental image and provides a detailed interference pattern of the interacting fields on the Ag crystal surface. To interpret the formation of the complex beating pattern, we indicate in Figure 4.8 the $k$-vectors for the beating pattern and the SPP fields, $k_{B}$ and $k_{s p p}$, by the red and green arrows, respectively. The simulation indicates that SPP wave packets are launched from most of the edges of the crystal, and their $k$ vector is determined by the edge orientation with respect to the incidence direction of excitation 
light. Particularly, when light interacts with an island edge, the direction of $k_{s p p}$ is determined by the external field in-plane $k$-vector according to the Snell's law of refraction, where the refracted beam is the SPP fields.[35] The appropriate refractive index can be obtained from the experimental dielectric constants for Ag.[1]

Specifically, $\gamma=0$ and $180^{\circ}$ for the front and back edges that are perpendicular to the incoming $k$-vector. The rear oblique coupling edges have $\gamma_{\text {rear }}= \pm 13.6^{\circ}$ and therefore are responsible for the tilted interference fringes. For the near-oblique edges, however, there is no emanating interference pattern, and parts of the crystal nearest to these edges are dark. This is because light, with energy $h v=3.21 \mathrm{eV}$, propagating from vacuum to Ag experiences total internal reflection, that is, the refracted SPP wave is evanescent. Combining the $k$-vectors from the different edges, we obtain vectors of the interference patterns (red arrows), which are perpendicular to the crystal edges and the interference wave fronts. The interference patterns give a false impression that waves are emitted parallel to the crystal edges, rather than the true SPP propagation, which follows from the momentum conservation as expressed in Snell's law. 


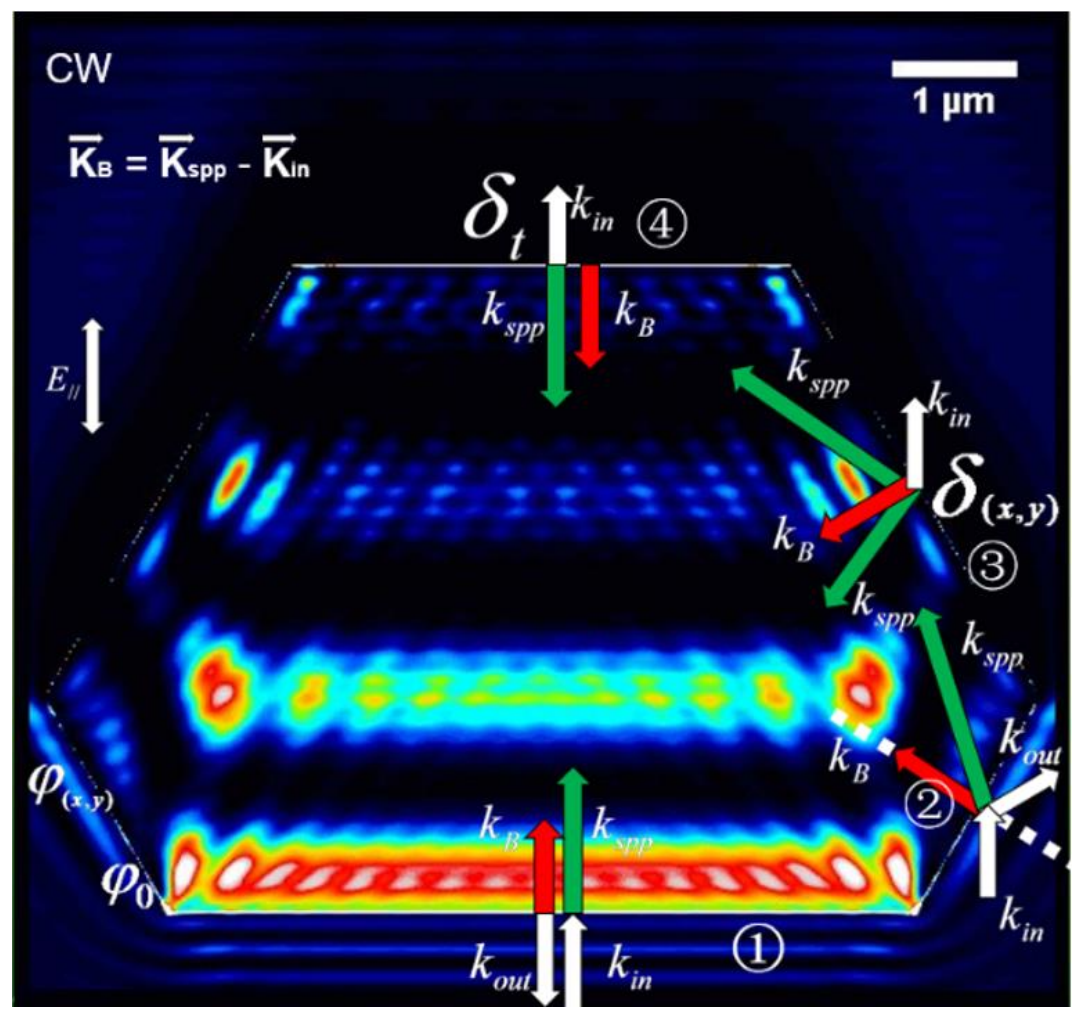

Figure 4. 8 Simulated PEEM image of SPPs excited on ain a truncated triangular $\operatorname{Ag}(111)$ island, excited with p-polarized $h v=3.21 \mathrm{eV}$ light. $\boldsymbol{k}_{i n}$ is the k-vector of incident light, $\boldsymbol{k}_{s p p}$ is the $k$-vector of the SPP field and $k_{B}$ is the k-vector of the beating field. $\varphi$ indicates the phase of external light at edges that can couple forward propagating SPP waves, $\delta$ indicates the phase of external light at edges that can couple back-propagating SPP waves. (1)-(4) indicate the the island edges that couple to SPP field in the sequence of excitation time. $E_{\|}$ indicates the in-plane polarization of the external light. 


\subsubsection{Dynamics of the Propagating SPPs}

To get more insight into dynamics of SPPs excited in 2D systems, we performed ITRPEEM measurements on a $\operatorname{Ag}(111)$ crystal for $h v=2.70 \mathrm{eV}$. Figure 4.9 (a), (b) shows ITR-PEEM images for double pulse pump-probe excitation for delays between the pulses of $\Delta t=0-12 \times 2 \pi$ $=18.3 \mathrm{fs}$, where one optical cycle corresponds to $1.52 \mathrm{fs}$. When the pump and probe pulses overlap, the imaging corresponds to the single-pulse excitation. The interference fringes are more pronounced at the near-edge (bottom) of the crystal where the SPP modes originate. After a delay of $18.3 \mathrm{fs}$, the fringes have better contrast and are now clearly observable on the far side of the island. For $\Delta \mathrm{t}=0$, the image contrast is produced by self-interference, where the same pulse generates the SPP wave packet and interferes with it on the time scale that the two fields overlap. For $\Delta \mathrm{t}=18.3 \mathrm{fs}$, each pulse creates its own SPP wave packet and interferes with it, but in addition to the self-interference, there is also cross-interference where the probe pulse interferes with the SPP of the propagated pump SPP wave packet. Such cross-interference imaging is already evident in the simulation of Fig. 3.5. The propagation explains the relatively stronger interference at the far end of the Ag island in Figure 4.9 (b). The total field is stronger at the far end after the delay because the pump SPP can propagate far enough to make a contribution to it. For a single-pulse measurement, the fringe contrast is determined by the excitation bandwidth if the intrinsic phase memory of the experimental probe is longer than the pulse duration. As the pump-probe pulse duration increases within the material dephasing time, for the in-phase excitation, the constructive interference will be strongest at the carrier frequency, and the frequency components in the spectral wings will experience destructive interference. That is because the Fourier transform bandwidth of an in-phase pulse pair is narrower than that of a single pulse. 

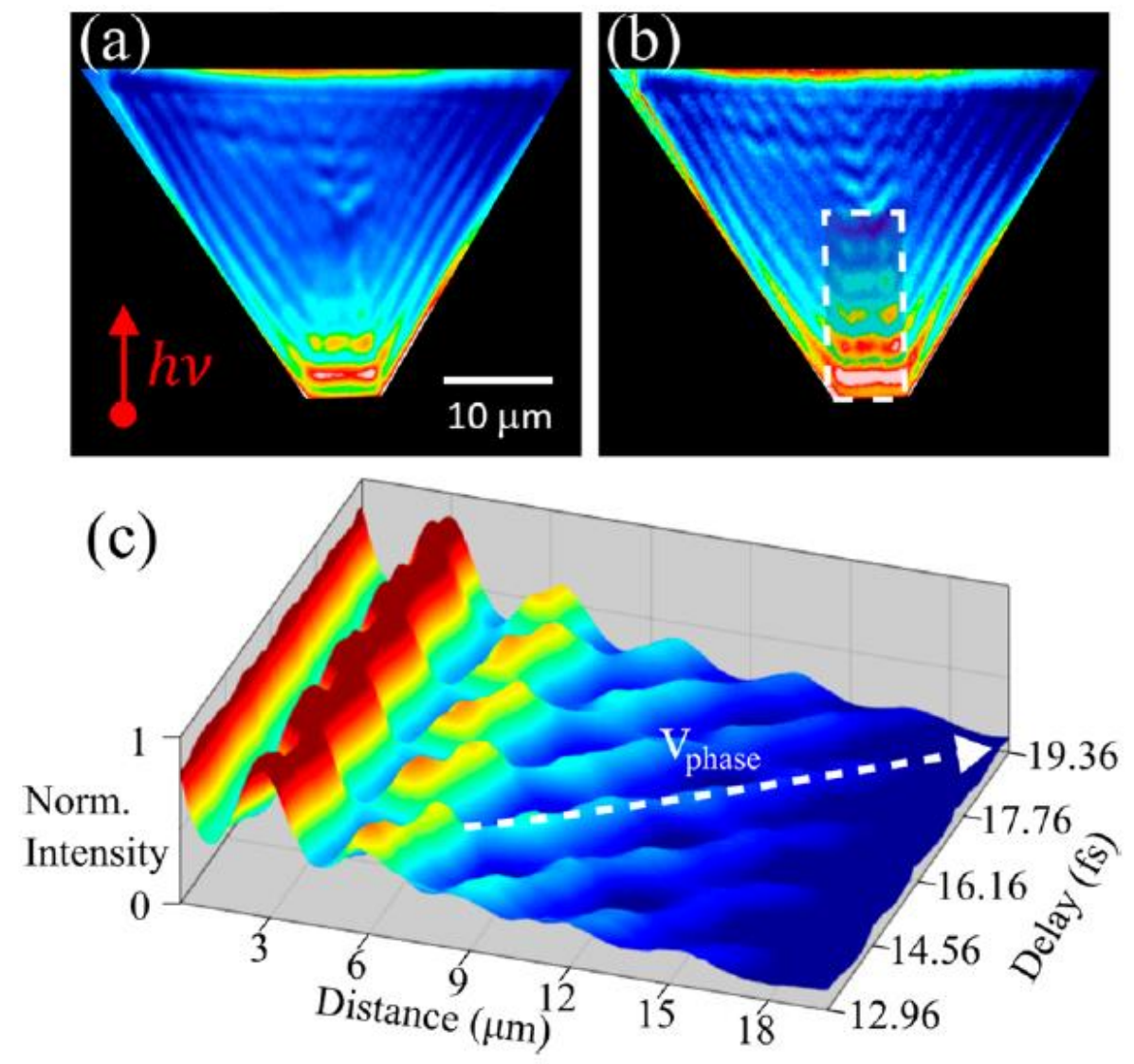

Figure 4. 9 ITR-PEEM images from a truncated triangle $\operatorname{Ag}(111)$ island obtained by double-pulse pumpprobe excitation at $h v=2.7 \mathrm{eV}$ with delays between the pulses of $\Delta t=0$ (a) and $\Delta t=18.3 \mathrm{fs}$ (b). The intensity of (b) is multiplied by a factor of 10 for better visibility. (c) 3D intensity plot integrated in the horizontal direction over part of the island that is indicated by the dashed rectangle in (b) in a selected delay time window spanning 6.4 fs. Clear progression of the beating maxima reveals the motion of SPP waves. The slope of the beating evolution marked by the dashed arrow gives the SPP phase velocity. 
The time- and space-dependent interference patterns provide nano-femto information on the SPP dynamics, including dispersive propagation and dephasing. To extract quantitative information on the SPP propagation in Figure 4.9 (c), I plot the PEEM line profiles integrated in the region, which is indicated by a rectangle in Figure 4.9 (b), as a function of delay time over a 6.4 fs range. The image shows a clear propagation of beating maxima away from the near-coupling surface as the delay is increased. The slope showing the propagation of constructive maxima is determined by the difference in the phase velocities of the SPP field and the external light. Near the coupling edge, the interference maxima oscillate back and forth because the intensity is dominated by the self-interference, which appears to oscillate due to a smaller contribution from the delay-varying cross-interference. Further from the coupling structure, however, the beating maxima are dominated by the cross-interference and therefore propagate. Such behavior is characteristic of all ITR-PEEM imaging. Tracing out the propagating beating maxima (the white dashed line in Figure 4.9 (c)) gives the SPPs phase velocity through the equation $V_{\text {phase }}=$ $S_{\text {trace }} \times c_{\|} /\left(S_{\text {trace }}+c_{\|}\right)$, where $S_{\text {trace }}$ is the slope of the maxima. In the case of the $h v=2.70 \mathrm{eV}$ excitation, the phase velocity is obtained to be $0.95 \mathrm{c}$, which is close to the expected value of $0.94 \mathrm{c}$ from the dielectric function of Ag. The SPP propagation is also affected by the wave packet group velocity as well as damping, but the Ag crystal size in Figure 4.5 is too small to propagate the SPP wave packet sufficiently far so that these effects can be accurately determined. 


\subsection{Spin Angular Momenta and Chirality of SPPs}

In this chapter, I will expand the discussion of SPPs coupling and distribution on 2D single crystal Ag island, by introducing a new degree of freedom for the excitation light, i.e. the polarization state. I will describe how changing the polarization of light affects the SPP excitation probability on the Ag surface. This is caused by differently polarized components of light coupling differently to the transversely or longitudinally polarized components of SPP fields. This effect depends on the driving field orientation with respect to components of the SPP field. The excited SPP field distribution for excitation with any polarized light can be considered as a superposition of two SPP modes that are coupled in different ways. Such coupling decomposition can explain properties of SPP field distributions, such as the plasmonic quantum spin hall, which is a consequence of spin-momenta matching between that of SPPs and that of light. Furthermore, the SPP dynamics following excitation with various polarizations of external light remain unchanged, even though their initial amplitudes and phases change.

\subsection{Spin-Angular Momentum (SAM) coupled SPPs on Ag Island}

\subsubsection{Polarization Dependent SPP Excitation and PEEM Imaging}

The geometry of the silver island discussed in this section is shown in Figure 5.1, which shows a PEEM image illuminated by the UV lamp. Different edges of the island are labeled by Greek letters. 


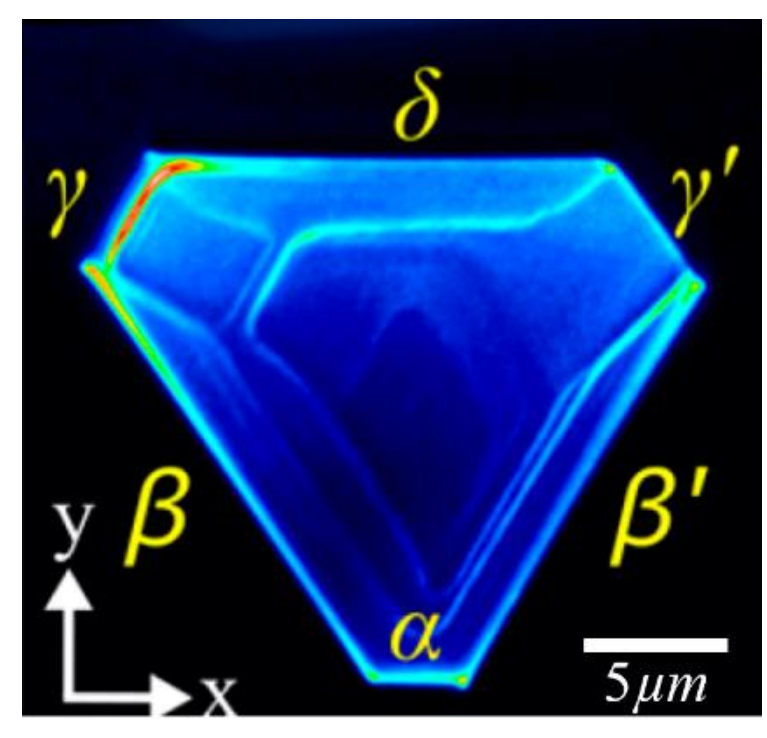

Figure 5. 1 1P-PEEM image of the Ag(111) surface of a single crystal island. The Greek letters label the edges of the island.

Before discussing experimental results, I first introduce the notation used in this section. The transverse and longitudinal components of the polarization fields at a coupling edge are labeled as $\boldsymbol{P}_{t} \sim \varepsilon_{0} \chi_{A g} \boldsymbol{E} \cdot \widehat{\boldsymbol{n}}_{\perp}$ and $\boldsymbol{P}_{l} \sim \varepsilon_{0} \chi_{A g} \boldsymbol{E} \cdot \widehat{\boldsymbol{n}}_{\|}$, which are tuned by the light polarization. In this chapter, $\boldsymbol{E}$ is the incident field, and $\widehat{\boldsymbol{n}}_{\perp}$ and $\widehat{\boldsymbol{n}}_{\|}$are unit vectors normal and parallel to the $\operatorname{Ag}(111)$ surface, respectively, as shown in Figure 5.2. In the case of linearly polarized light excitations, the two basic excitation polarizations are $p$ - and s-polarization, which are denoted in Fig. 5.2 as the blue arrows. Note that $\boldsymbol{P}_{t}$ and $\boldsymbol{P}_{l}$, which are determined by normal and parallel projections of the incident field onto the surface, $\boldsymbol{E}_{\perp}=\boldsymbol{E} \cdot \widehat{\boldsymbol{n}}_{\perp}$ and $\boldsymbol{E}_{\|}=\boldsymbol{E} \cdot \widehat{\boldsymbol{n}}_{\|}$, evolve into propagating SPP fields, which contain interconverting transverse and longitudinal components, which evolve into each other from the point of coupling as SPPs propagate. Their complex wavevectors and propagation from the coupling edges is defined by complex wave vectors, $\widetilde{\boldsymbol{k}}_{s p p}=k_{x} \hat{x}+k_{y} \hat{y}+i \kappa \hat{z}$ through 
the Snell's law: they propagate on the Ag crystal for several microns, until they encounter another edge, whereupon they reflect or refract.

With circularly polarized light, which can be obtained by superposition of $p$ - and $s$ polarized light as stated in Fig. 5.2, I will describe how the in-plane spin angular momentum (SAM) of light projected onto the sample surface $\left(\boldsymbol{S}_{\|}^{L}\right)$, marked by red arrows in Fig. 5.2, influences the SPP properties. Particularly, $\boldsymbol{S}_{\|}^{L}$ interacts with the transverse SAM of SPPs $\left(\boldsymbol{S}_{\perp}^{s p p}\right)$, which is orthogonal to the $k$-vector $\left(\widetilde{\boldsymbol{k}}_{\text {spp }}\right)$ of the SPP fields at the sample surface, to influence the effectiveness of launching SPPs depending on the handedness of light. Such helicity dependent selectivity of SPPs generation is due to an additional constraint, $\boldsymbol{S}_{\|}^{L} \cdot \boldsymbol{S}_{\perp}^{s p p}$, where the subscripts emphasize that for light (SPPs) it is parallel (perpendicular) to their $k$-vectors.
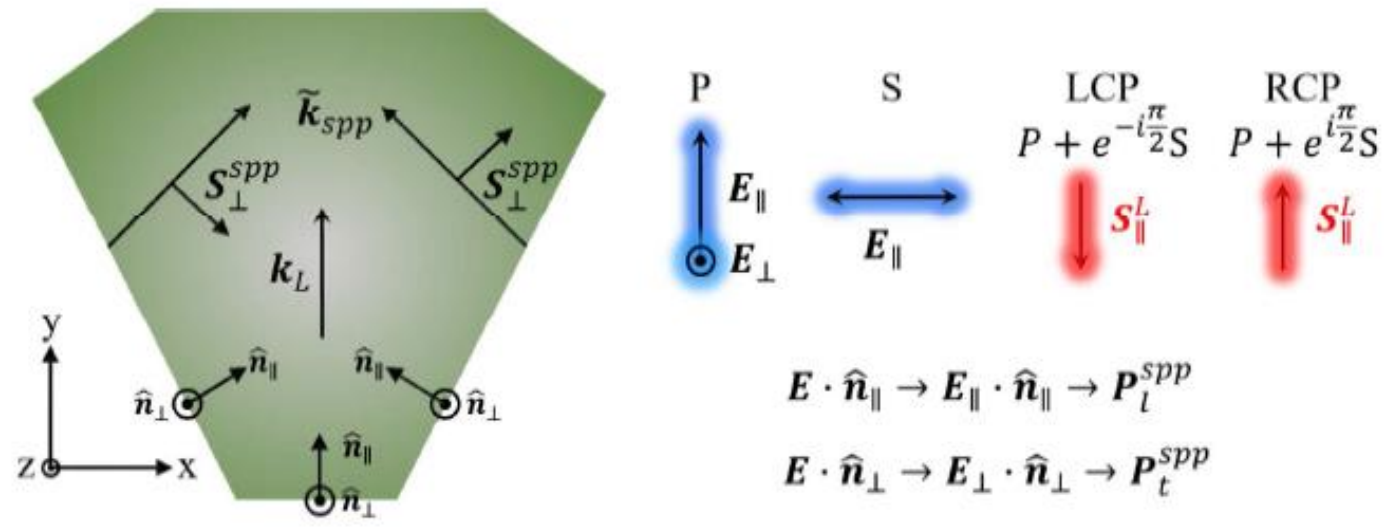

Figure 5. 2 Annotations of vectors and related physical quantities. $\widehat{\boldsymbol{n}}_{\perp}$ and $\widehat{\boldsymbol{n}}_{\|}$are unit vectors normal and parallel to sample surfaces. $\widetilde{k}_{s p p}$ is the k-vector of the SPP wave, $\boldsymbol{k}_{L}$ is the in-plane $k$-vector of the excitation light. $S_{\perp}^{s p p}$ is the transverse SAM of the SPP fields, and $S_{\|}^{L}$ is the in-plane SAM of the external light field. $E_{\|}$ and $E_{\perp}$ are the in- and out-of-plane components of the external field, respectively. 
The observed beating patterns for $p$ - and s-polarized light are shown in Figure 5.3; they are similar to those in the Chapter 4 in the case of the p-polarized light. The measured beating period within the island starting from the $\alpha$-edge (for the edge designations see Figure 5.1) is $\lambda_{B}=$ $3.45 \mu \mathrm{m}$, in agreement with the calculated value of $\lambda_{B}=3.48 \mu \mathrm{m}$ from a Drude parameterization of the dielectric function of Ag.[5, 70] Because the $\alpha$-edge has a finite length, this beating pattern close to the coupling edge follows Fresnel diffraction, and as the waves reach to far field, the SPP waves get more intese at the center and form the diffraction maximum in the regions of the Fraunhofer diffraction, which is beyond the Ag island. [74]
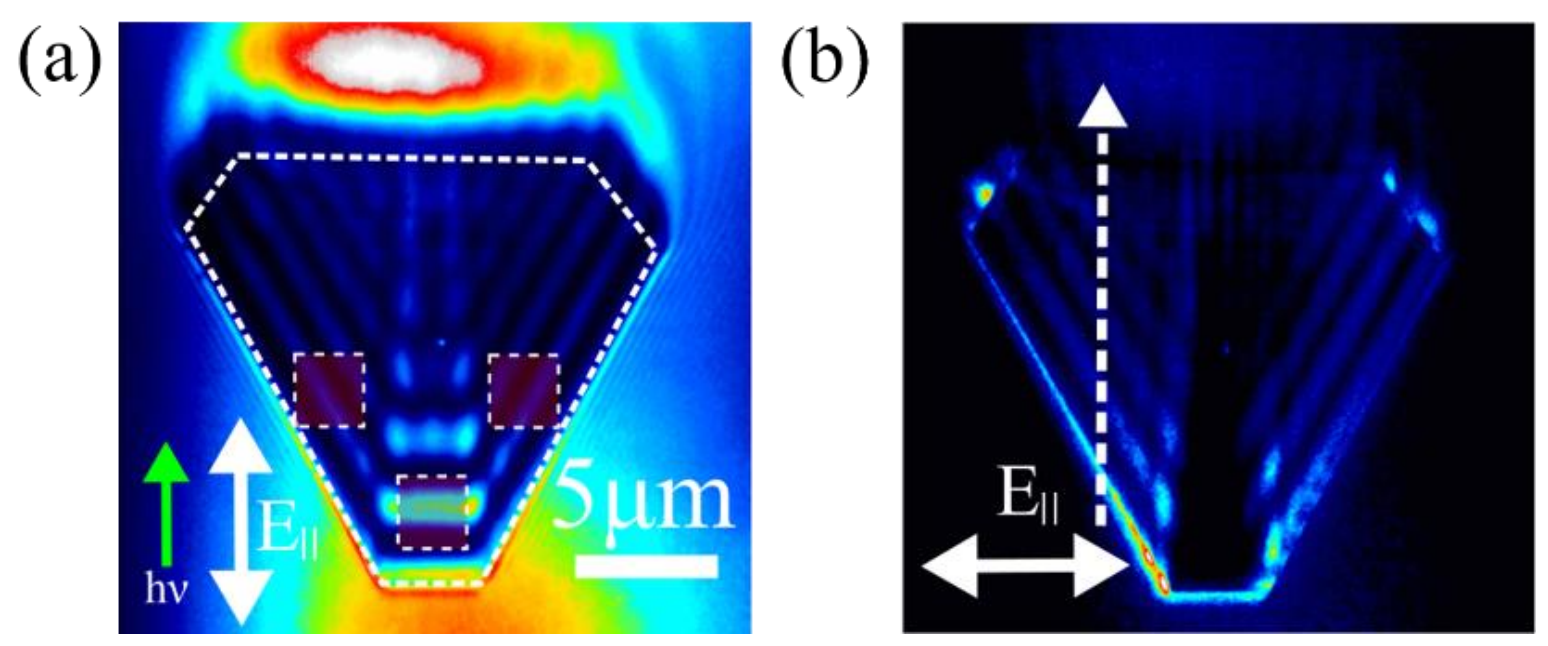

Figure 5. 3 PEEM image of the truncated Ag island excited by hv $=\mathbf{2 . 7 0} \mathrm{eV}$, for $p$ - and $s$-polarized light excitations.

As is evident in Figure 5.3 (b), no beating pattern is observed from $\alpha$ edge with $s$-polarized light, because the in-plane field of the external light is parallel to the $\alpha$ edge. In addition, the SPPs 
beating patterns emanating from the $\beta$ and $\beta^{\prime}$ edges are out-of-phase. This is a result of the excitation symmetry, since charge oscillations will have the same behavior at both edges, which are mirror symmetric, but are driven by $\boldsymbol{E}_{\|}$in the opposite direction. Thus, the fields pointing inward from the sides of the island need to be out-of-phase, similar to a dipole distribution.

Using a half-waveplate (HWP) and a quarter-waveplate (QWP), we could achieve the full rotation of the external light polarization to get various linear and elliptical polarizations. Figure 5.4 shows the 2P-PEEM images obtained for linear polarizations, where $\boldsymbol{E}_{\|}$is rotated to be normal to either the $\beta$ or $\beta^{\prime}$ edges. The observed PEEM images are essentially asymmetric with respect to the island symmetry axis. In Figure 5.4(a), when $\boldsymbol{E}_{\|}$is perpendicular to $\beta$ edge, strong SPPs beating pattern is seen on the left side of the Ag island, and a weaker one is observed on the right side. The opposite photoemission distribution is observed when the $\boldsymbol{E}_{\|}$is perpendicular to the $\beta^{\prime}$ edge. Such asymmetry with respect to the rotation of linear polarization is a consequence of the extent of field projection normal to the coupling edge. Because if the excitation field is parallel to the coupling edge, SPPs coupling is inherently inefficient, similar as SPPs coupling at the $\alpha$ edge with s-polarized excitation, where the in-plane field component is parallel to the $a$ edge. 

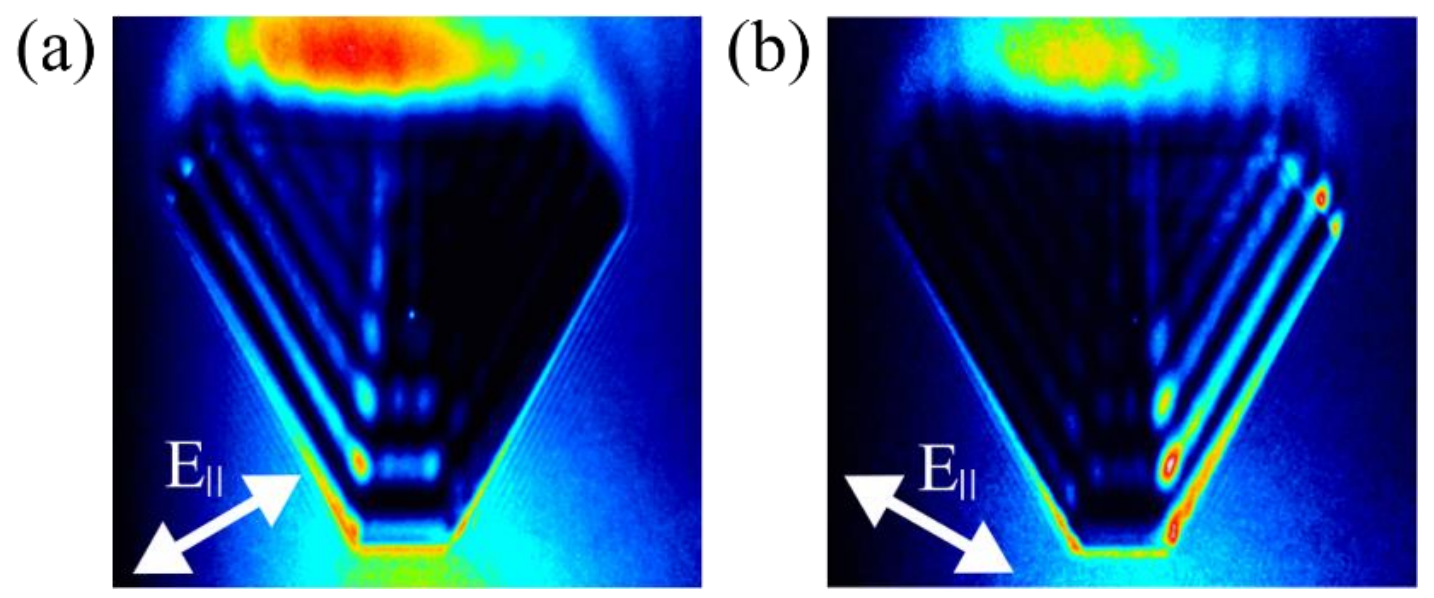

Figure 5. 4 PEEM image of the truncated Ag island at $460 \mathrm{~nm}$, for selected linearly polarized light excitations.

When the Ag island is illuminated with circularly polarized light, the PEEM images reveal similar asymmetry in the beating patterns, as in the case of the linear polarization, as shown in Figure 5.5. For left circularly polarized light, SPPs coupled from the $\beta$ edge is distinct, while the SPPs from $\beta^{\prime}$ is weak. Once the handedness of the excitation light is reversed, mirror image of the SPPs interference pattern is observed, where SPPs are pronounced from the $\beta^{\prime}$ edge and SPPs from $\beta$ edge is suppressed. The mechanism of the asymmetry of PEEM images is a result of the coupling between the transverse spin angular momentum of the SPPs and the spin angular momentum of external light. Particularly, for left circularly polarized light, the in-plane projection of the SAM of light $\boldsymbol{S}_{\|}^{L}$ points $-y$ in Fig. 5.5 (a), as indicated by the red arrow. While the SPP waves from $\beta$ and $\beta^{\prime}$ edges carry transverse $\mathrm{SAM} \boldsymbol{S}_{\perp}^{s p p}$, as indicated by the yellow arrows, with opposite sign when projected along the $y$ direction. As a result, the dot product $\boldsymbol{S}_{\|}^{L} \cdot \boldsymbol{S}_{\perp}^{s p p}$ favors the excitation of SPPs from the $\beta$ edge, and suppress the excitation from the $\beta^{\prime}$ edge. In the case of right circularly polarized light in Fig 5.5 (b), the coupling preference is a mirror image of Fig. 5.5 (a) with respect 
to the $y$-axis, because of the handedness of the external light is reversed. A detailed explanation of the enhancement/suppression will be provided in later sections using a picture of wave superpositions.
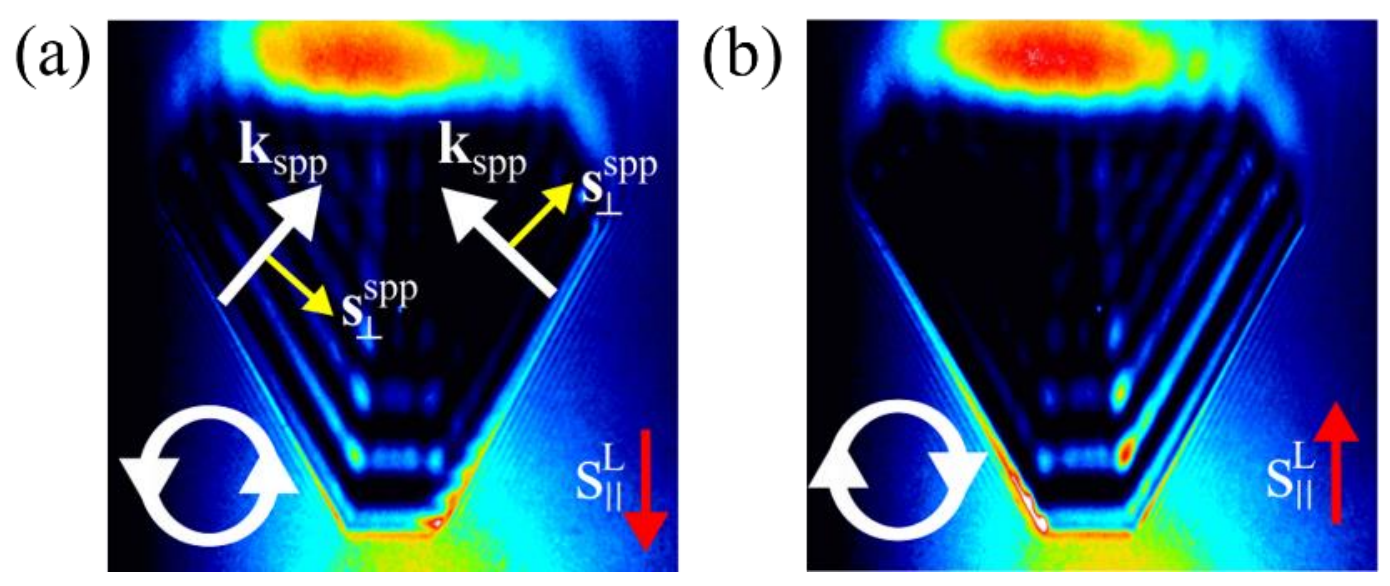

Figure 5. 5 PEEM image of the truncated Ag island at $460 \mathrm{~nm}$, for left and right circularly polarized light excitations.

To confirm the correctness of our observed PEEM images of both linearly and circularly polarized light, we performed 3D-FDTD simulations for the same excitation conditions. The resulting PEEM images are shown in Figure 5.6. Most of the calculated PEEM images reproduce well the experimental findings. A small discrepancy is found for the s-polarized excitation, where a weak SPP interference pattern is observed in experiment, but it does not appear in the FDTD simulation. This is because the incident pulse in experiment has a residual $\boldsymbol{E}_{\perp}$ component due to imperfect alignment, whereas in the simulation, the incident light is perfectly s-polarized, and thus no pronounced SPPs beating pattern should exist due to the lack of the $\boldsymbol{E}_{\perp}$ field, which interferes 
with the SPP field on top of the island. The discrepancy could be also due to coupling of orthogonal field components, which is not included in the FDTD simulation but may contribute to the signal. The photoemission yield and phase analysis in the following sections will be based on the numerically simulated results shown here.
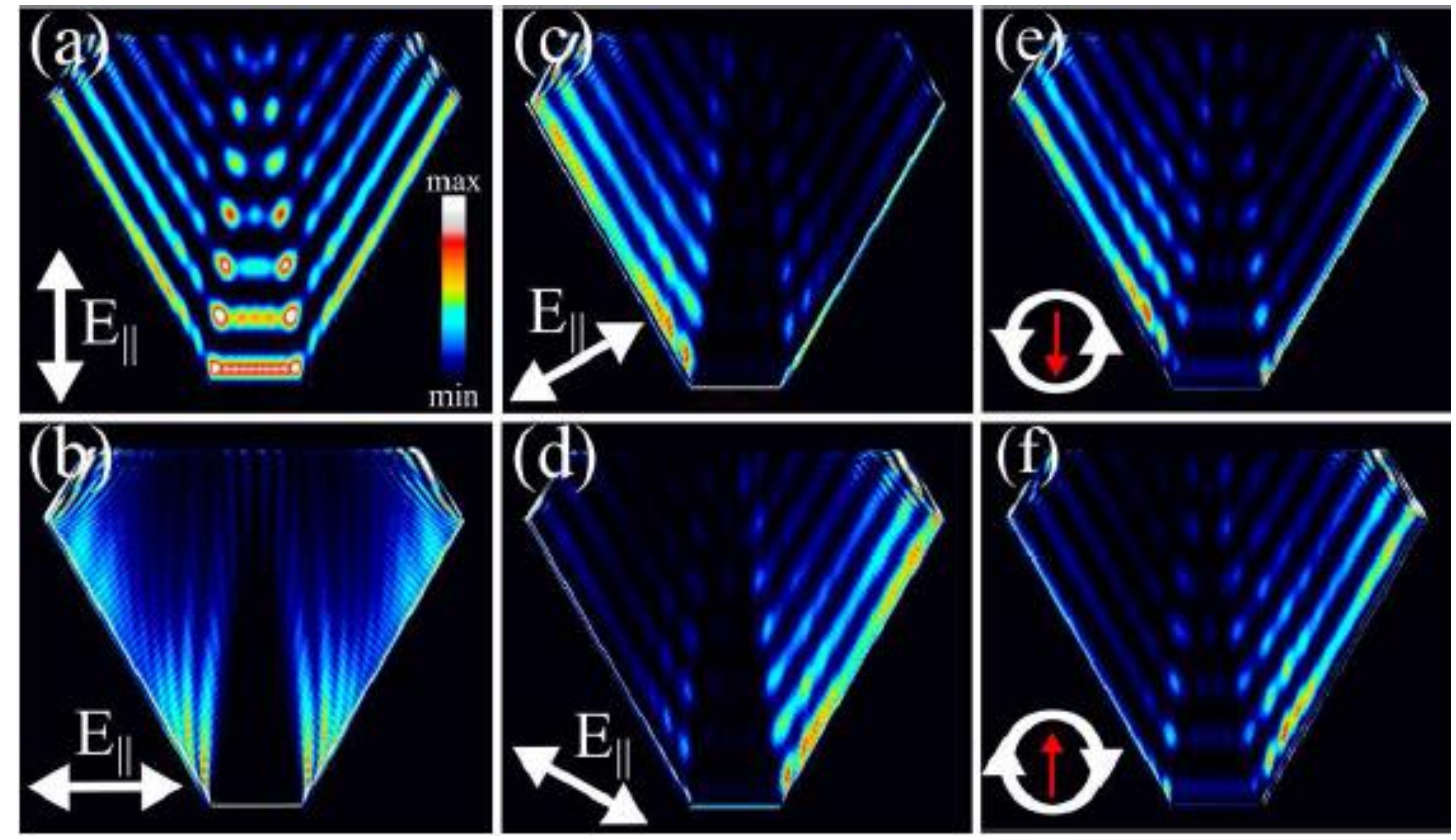

Figure 5. 6 Simulated 2PP PEEM images of the Ag island at $460 \mathrm{~nm}$ excitation under linear (a-d) and circular (e-f) excitations, showing the asymmetric interference patterns. The color scale is normalized to (a).

Besides fully vectorial FDTD simulations, we also performed Huygens principle based analytical simulations to confirm the PEEM images in the case of circularly polarized light. And the details of the analytical model can be found in the numerical method section. The corresponding simulation for the Ag island is constructed with the same geometry as in the FDTD simulations. And the circular polarization is simulated by combining two orthogonally linearly 
polarized light pulses. The resulting 2P-PEEM images are shown in Figure 5.7, for left and right polarized light excitation. Clear PEEM yield asymmetry is observed similar as in Fig. 5.5 and Fig 5.6. Both the FDTD simulation and the analytical simulation show that the asymmetry is associated with the enhancement of the SPPs excitation on one side of the island, and the suppress of the SPPs excitation on the other side.

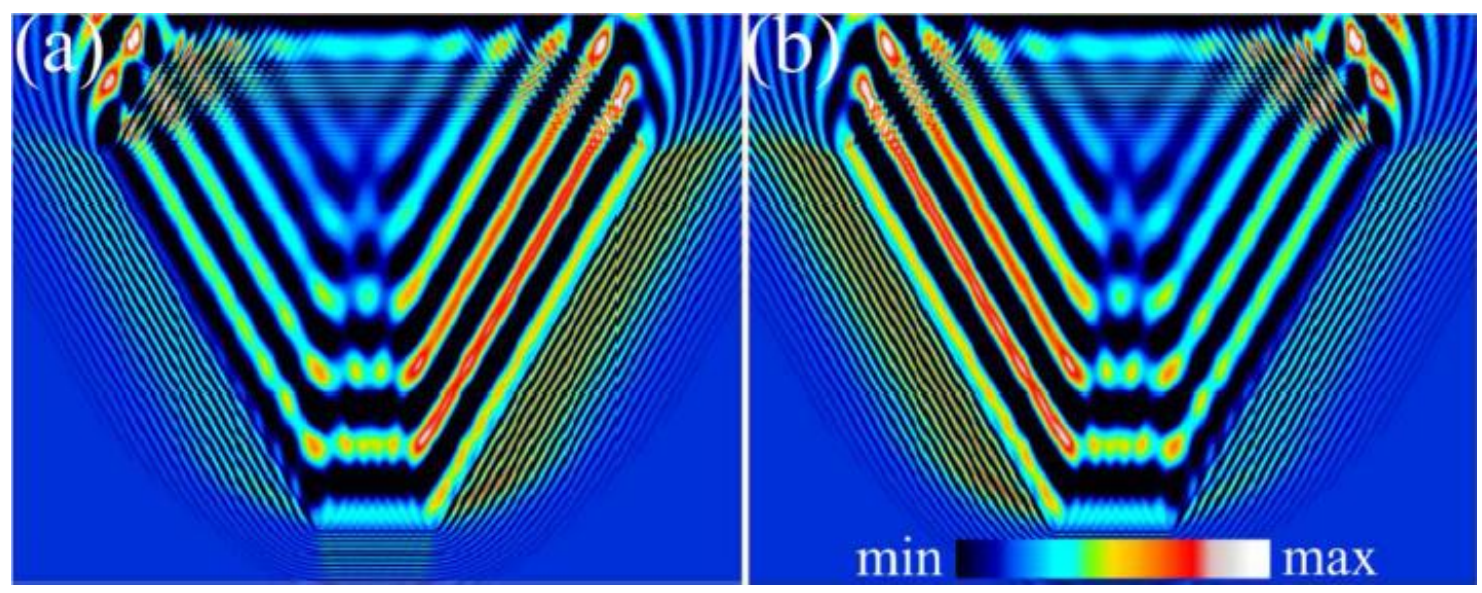

Figure 5.7 (a)(b) Simulated 2P-PEEM images based on Huygens-Fresnel model, for left and right polarized light excitations, respectively.

\subsubsection{Polarization Dependent Photoemission Yield}

More information can be extracted from the PEEM images by analyzing the photoemission yield within specific regions of the sample as a function of polarization states of light. Although the linear and circular polarizations produce superficially similar asymmetries as discussed in the previous section, differences due to the SAM matching for the circularly polarized excitations can 
be evaluated by quantifying the waveplate angle $\xi$ dependent 2PP yields from selected regions of the Ag crystal. The angle $\xi$ refers to the angle of HWP for linear polarizations, or QWP for elliptical polarizations, respectively. Figure 5.8 shows the experimental and FDTD simulation of polarization dependence of $2 \mathrm{PP}$ yields, which are obtained by integrating the signal over the indicated square regions in Figure 5.3, upon rotation of the retardation plate angle $\xi$. At the center of the island, where SPPs are excited from the $\alpha$-edge [Fig. 5.8 (a)(b)], the $\xi$ dependences of 2PP yields have symmetric dumbbell shapes because the out-of-plane component of the total polarization field, $\boldsymbol{P}_{t o t}(x, y, \tau) \cdot \widehat{\boldsymbol{n}}_{\perp}$ defines the $2 \mathrm{PP}$ yield for both the linear and circular polarizations (The arrows in Figure 5.8 give polarizations for different $\xi$ ). For linear polarization, the maximum of the photoemission happens for p-polarized light, $\xi=0, \pm \frac{\pi}{2}$, and the minimum for s-polarized light, $\xi= \pm \frac{\pi}{4}$, with a $\cos ^{4} \xi$ dependence of $2 \mathrm{PP}$ yields. This indicates that both launching of SPPs and photoemission are driven by $\boldsymbol{E}_{\perp}$, because $\boldsymbol{E}_{\perp}=0$ for $s$-polarized light, However, for circularly polarized light, the $2 \mathrm{PP}$ yield is not extinguished at $\xi= \pm \frac{\pi}{4}$, because the external field always includes $\boldsymbol{E}_{\perp}$. 

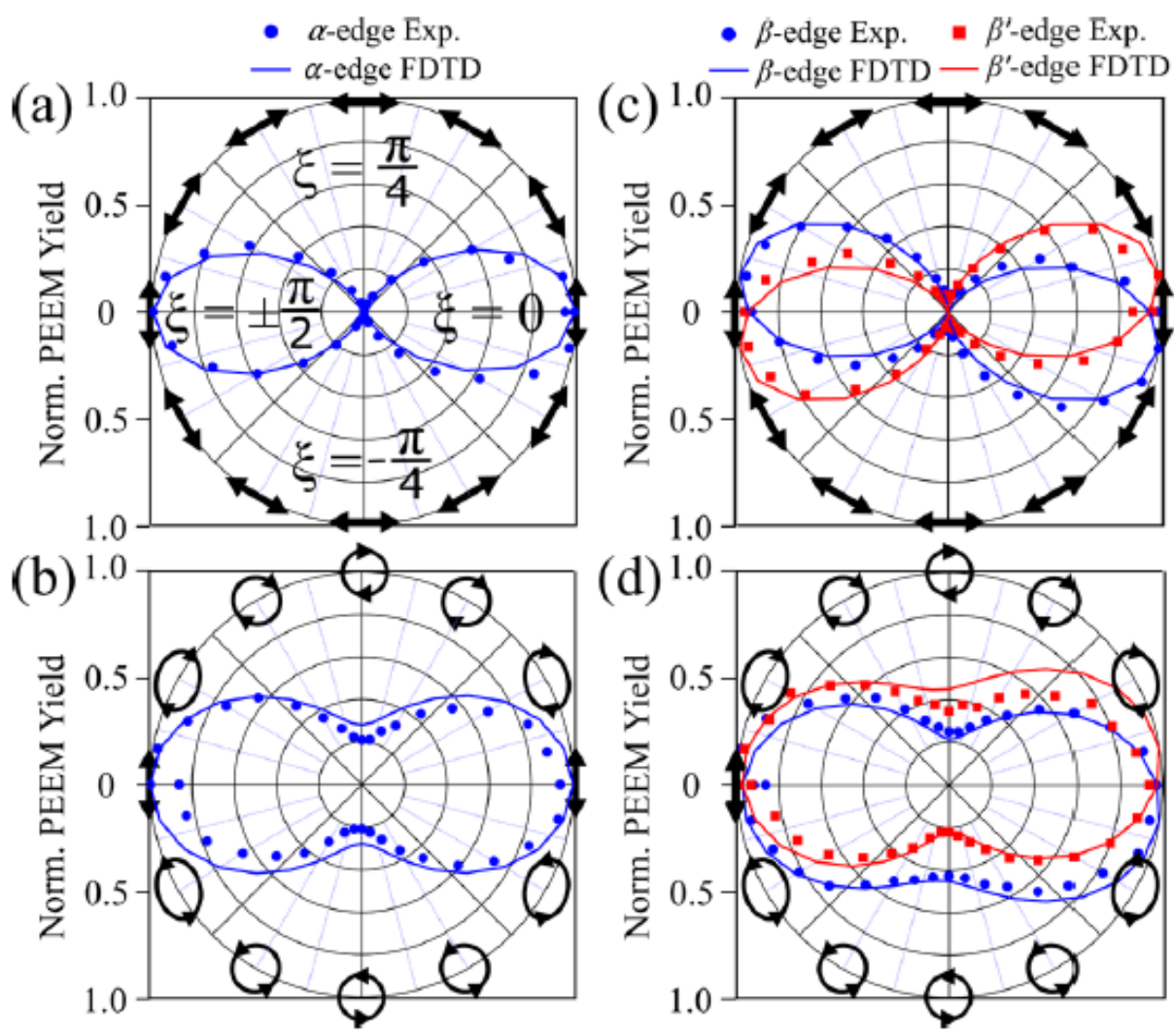

Figure 5. 8 Circular plots of the experimental (makers) and FDTD simulated (lines) 2PP yields from the regions indicated in Figure 5.3(a) as a function of $\xi$. The radial axis length represents normalized PEEM yield, while the polar axis is the retardation plate angle $\xi$, where one cycle corresponds to $\pi$ rotation, for tuning of the linear and circular polarizations. The effective polarizations are also indicated for selected $\xi$. (a)(b) Normalized 2PP intensities from the coupling at the $\alpha$ edge (the central rectangle of Figure 5.3(a)) for the linearly and circularly polarized excitations. (c)(d) Linearly and circularly polarized excitation intensities taken from $\beta$ (blue) and $\beta^{\prime}$ (red) edges (Figure 5.3(a)), showing tilting of the distribution dumbbells by $\sim \pm 11^{\circ}$ and $\sim 5^{\circ}$, with respect to the $\alpha$ edge. The helicity dependent asymmetry is caused by directional coupling to SPPs due to the SAM matching.

Furthermore, in Figure 5.8(c) we consider the polarization dependent SPP excitation intensities at the $\beta$ and $\beta^{\prime}$ edges, by integrating the $2 \mathrm{PP}$ yields within the designated proximate left 
and right squares (Figure 5.3). The retardation plate angle (polarization) dependent 2PP yields are similar to those in Figure 5.8(a) for the $\alpha$ edge, but the distributions are tilted by $\pm 11^{\circ}$ for leftright-side coupling with respect to the meridional plane. Because $\boldsymbol{E} \cdot \widehat{\boldsymbol{n}}_{\perp}$ is maximum for ppolarization, while launching of SPPs is maximized when $\boldsymbol{E}_{\|}$is normal to the $\beta$ and $\beta^{\prime}$ edges, the maximum $2 \mathrm{PP}$ signal occurs at an angle that maximizes their joint contributions, i.e. $\boldsymbol{E} \cdot \widehat{\boldsymbol{n}}_{\perp}+$ $\boldsymbol{E} \cdot \widehat{\boldsymbol{n}}_{\|} \cdot$

The same experiment for rotating of the circular polarization in Figure 5.8(d) rotates the 2PP yield distributions, by $\pm 5^{\circ}$ with respect to that of Figure $5.8(\mathrm{~b})$. This left/right asymmetry arises from the preferred coupling at the $\beta^{\prime}(\beta)$ edge of light with the opposite chirality, and in addition produces an upward (downward) displacement of the centroid of 2PP distributions; the asymmetry defined by $A_{\text {circular }}=\frac{I_{L C P}-I_{R C P}}{I_{L C P}+I_{R C P}}$ is -0.26 , which we attribute to the SAM matching condition for the excitation of the spin-momentum locked SPPs.[11, 13, 15, 75] Because there is a fixed relationship between $\boldsymbol{k}_{s p p}$ and SAM of SPPs, the strength of the SPP excitation at the opposing island edges $\left(\beta\right.$ and $\beta^{\prime}$ ) depends on SAM matching according to the projection $\boldsymbol{S}_{\|}^{L} \cdot \boldsymbol{S}_{\perp}^{\text {spp }}$. Specifically, when the SAM of LCP light is anti-parallel to its $k$-vector, its projection onto the surface, $\boldsymbol{S}_{\|}^{L}$, has a component pointing to the negative $y$ direction (Figure 5.3); consequently, the SPP coupling is favored at the $\beta$ edge where $\boldsymbol{S}_{\|}^{L} \cdot \boldsymbol{S}_{\perp}^{s p p}$ is larger than at the $\beta^{\prime}$ edge.

To better illustrate the field contributions, an analytical model for the polarization dependent photoemission is proposed. The excitation of the SPPs can be described by $\boldsymbol{P}_{l} \propto$ $\varepsilon_{0} \chi_{A g}\left(\boldsymbol{E}_{x}+\boldsymbol{E}_{y}\right) \cdot \widehat{\boldsymbol{n}}_{||}$for the longitudinal component, and $\boldsymbol{P}_{t} \propto \varepsilon_{0} \chi_{A g} \boldsymbol{E}_{z} \cdot \widehat{\boldsymbol{n}}_{\perp}$ for the transverse component. Here $\boldsymbol{E}_{x}, \boldsymbol{E}_{y}$, and $\boldsymbol{E}_{z}$ are the electric field components of the incident field, $\widehat{\boldsymbol{n}}_{\|}$is the 
unit vector perpendicular to the coupling edge in the plane of the metallic surface, $\widehat{\boldsymbol{n}}_{\perp}$ is the unit vector normal to the surface. Notations are referred to Figure 5.2.

Note, that $\boldsymbol{P}_{l}$ and $\boldsymbol{P}_{t}$ combine with the appropriate phase to give the excited SPP field, which oscillates between the transverse and longitudinal polarizations as it propagates. In other words, they are not independent quantities, but light couples to these components differently. In addition, from the numerical simulation, the SPP field excited by $p$-polarized $\left(\boldsymbol{E}_{x}=0\right)$ and $s$ polarized light $\left(\boldsymbol{E}_{y}=\boldsymbol{E}_{z}=0\right)$, which are dominated by $\boldsymbol{P}_{t}$ and $\boldsymbol{P}_{l}$ respectively, have a relative phase depending on the coupling geometry. Therefore, we introduce a phase factor $\boldsymbol{\varphi}_{\boldsymbol{c}}$ for SPPs coupling between the $p$ - and $s$-polarizations. Specifically, for pure $s$-polarized excitation, a phase term is added on the $\boldsymbol{E}_{x}$ component, i.e. $\boldsymbol{P}_{l} \propto \varepsilon_{0} \chi_{A g}\left(\boldsymbol{E}_{x} \boldsymbol{e}^{i \boldsymbol{\varphi}_{c}}+\boldsymbol{E}_{y}\right) \cdot \widehat{\boldsymbol{n}}_{\|} \cdot$

To account for the polarization dependent photoemission yield in the case of linear polarization, we consider the $\mathrm{z}$ component of the total polarization field exclusively, because the in-plane components give much weaker $2 \mathrm{PP}$ signal. Therefore, the $2 \mathrm{PP}$ yield can be written as $Y_{\text {lin }} \propto \int_{-\infty}^{t}\left(\boldsymbol{P}_{\text {tot }} \cdot \widehat{\boldsymbol{n}}_{\perp}\right)^{4} d \tau \propto \int_{-\infty}^{t}\left[\left(\boldsymbol{P}_{l}+\boldsymbol{P}_{t}+\varepsilon_{0} \chi_{A g} \boldsymbol{E}\right) \cdot \widehat{\boldsymbol{n}}_{\perp}\right]^{4} d \tau$. The SPP polarization components in the expression are determined by the incident field components, which are varied $\mathrm{t}$ as a function of $\xi\left(\xi=0\right.$ corresponds to $p$-polarization) through the Jones matrix for a $\frac{\lambda}{2}$ plate:

$$
\begin{gathered}
\boldsymbol{E}_{\boldsymbol{x}}(\xi)=\boldsymbol{E} \sin 2 \xi \\
\boldsymbol{E}_{\boldsymbol{y}}(\xi)=\boldsymbol{E} \cos 2 \xi \cos \boldsymbol{\theta} \\
\boldsymbol{E}_{\boldsymbol{z}}(\xi)=\boldsymbol{E} \cos 2 \xi \sin \boldsymbol{\theta}
\end{gathered}
$$

These expressions give:

$$
Y_{\text {lin }} \propto \int_{-\infty}^{t}\left[E\left(\left(\sin 2 \xi e^{i \varphi_{c}} \widehat{x}+\cos 2 \xi \cos \theta \widehat{y}\right) \cdot \widehat{n}_{\|}+2 \cos 2 \xi \sin \theta\right)\right]^{4} d \tau
$$


or the $\xi$ dependence of the 2PP signal upon rotation of the linear polarization. Here the factor of two in the last term accounts for the combination of the z-component of the $\boldsymbol{P}_{t}$ and $\boldsymbol{E}_{\boldsymbol{z}}$, and $\boldsymbol{\varphi}_{c}$ is chosen to be the angle of the coupling edge with respect to the $x$-axis ( $60^{\circ}$ for $\beta$ edge).

In Figure 5.9(a)(c), I plot the experimental data of the photoemission yield for the $\alpha$ and $\beta$ edges as in Figure 5.8, along with the calculated curves given by the analytical expression above. we see a good agreement at both $\alpha$ and $\beta$ edges as the angle $\xi$ is rotated, where the tilting of the polarization dependence dumbbell with respect to horizontal axis is reproduced. Such tilting is induced by the dot product of the fields in the excitation of the longitudinal component of SPPs, as well as the introduced phase factor $\boldsymbol{\varphi}_{c}$ in eq. 5.4.
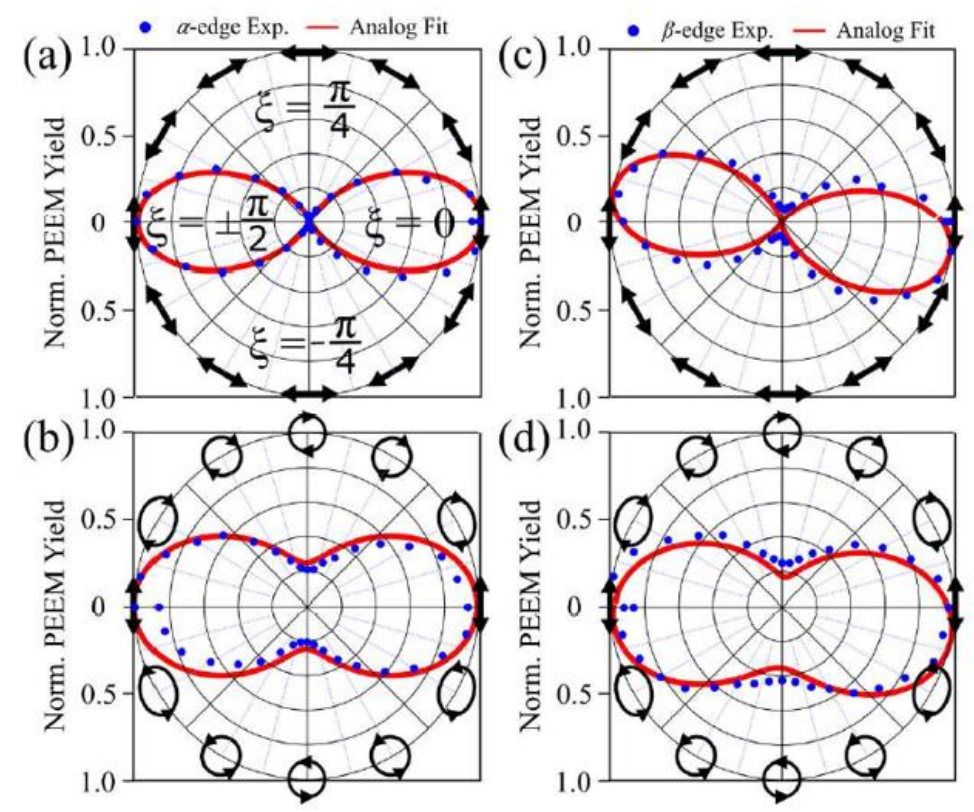

Figure 5. 9 Experimental data and analytical fitting of the experimental photoemission yields at various regions of interest in comparison with the numerical simulation results in Figure 5.8. (a)(b) show the 2PP yield taken at the central square region in Figure 5.3(a). (b)(d) show the 2PP yield in the squared region close to the $\beta$ edge in Figure 5.3(a). 
As for the $2 \mathrm{PP}$ signal with rotation of the $\frac{\lambda}{4}$ plate, which varies the elliptical polarization, we use similar approach to describe the total polarization field, but with a coupling efficiency term that accounts for the effect of SAM matching, $C_{S A M}$, which determines the asymmetry of the photoemission yield for light with opposite chirality, as observed in the experiment, i.e. $Y_{\text {circ }}(x, y) \sim \int_{-\infty}^{t}\left[\left(C_{S A M}\left(\boldsymbol{P}_{l}+\boldsymbol{P}_{t}\right)+\varepsilon_{0} \chi_{A g} \boldsymbol{E}\right) \cdot \widehat{\boldsymbol{n}}_{\perp}\right]^{4} d \tau$. Similarly, from Jones matrix, the incident field components that determine the SPP field can be expressed by (for the $\frac{\lambda}{4}$ plate, $\xi=0$ also corresponds to the p-polarization):

$$
\begin{gathered}
E_{x}(\xi)=E(1-\mathrm{i}) \sin \xi \cos \xi \\
E_{y}(\xi)=E\left(\cos ^{2} \xi+i \sin ^{2} \xi\right) \cos \theta \\
E_{z}(\xi)=E\left(\cos ^{2} \xi+i \sin ^{2} \xi\right) \sin \theta
\end{gathered}
$$

Therefore, the $2 \mathrm{PP}$ yield is written as:

$$
\begin{array}{r}
Y_{c i r c}(x, y) \propto \int_{-\infty}^{t}\left[\left(C_{S A M}\left(P_{l}+P_{t}\right)+\varepsilon_{0} \chi_{A g} E\right) \cdot \widehat{n}_{\perp}\right]^{4} d \tau \\
\propto \int_{-\infty}^{t \quad} \begin{array}{c}
\left.\left(\cos ^{2} \xi+\mathrm{i} \sin ^{2} \xi\right) \cos \theta \widehat{y}\right) \cdot \widehat{n}_{\| M}+ \\
\left.\left(\cos ^{2} \xi+i \sin ^{2} \xi\right) \sin \theta\right)+ \\
\left.\left.\left(\cos ^{2} \xi+i \sin ^{2} \xi\right) \sin \theta\right)\right] d \tau
\end{array}
\end{array}
$$

Note that the field component $\boldsymbol{E}_{\boldsymbol{z}}$ after passing through a $\frac{\lambda}{4}$ plate never reaches zero. In Figure 5.9(b)(d), I plot the 2PP yield at $\alpha$ and $\beta$ edges as a function of $\frac{\lambda}{4}$ plate angle $\xi$ for elliptically polarized light, along with the analytically calculated curves. By fitting the 2PP yield asymmetry for the opposite light ellipticities, we find the coupling term $C_{S A M}$ to be 0.55 . While $C_{S A M}$ determines the asymmetry of the photoemission yields between the left and right circularly polarized light, the dot product between the excitation field and the unit vector of the edge, as well as the relative phase $\boldsymbol{\varphi}$, determine tilting of the dumbbell distributions. 


\subsubsection{Polarization Dependent SPP Phases}

Having established the factors that determine the polarization dependent SPP excitation for both the linearly and circularly polarized light, next I will further analyze the SPP phases and amplitudes for different polarization states of $\boldsymbol{E}(x, y, t)$. We extract the initial phases $\Phi_{s p p}$ of SPPs from the experimentally measured locations of the beating maxima relative to the $\beta$ and $\beta^{\prime}$ coupling edges as $\xi$ is varied, and plot them in Figure 5.10(a)(b).

For the linearly polarized excitation, a distinct variation of $\Phi_{s p p}$ occurs with a period of $\xi=\frac{\pi}{2}$, which causes a continuous excursion of the SPP phase by $\pi$ as the polarization vector of $\boldsymbol{E}$ is rotated by $\pi$. An abrupt $\pi$ phase shift in of $\Phi_{s p p}$ occurs, however, when the excitation field is rotated through $s$-polarization. Because $s$-polarized light dominantly excites $\boldsymbol{P}_{l}$, which varies slowly, so the $\pi$ shift of $\Phi_{s p p}$ is caused by the abrupt $\pi$ shift of $\boldsymbol{P}_{t}$ as $\boldsymbol{E}_{\perp}$ passes through zero. Notably, the variation of the initial phase $\Phi_{s p p}$ is asymmetric with respect to direction of the polarization rotation $\xi$, and the asymmetry is reversed between the $\beta$ and $\beta^{\prime}$ edges [Figure 5.10(a)].

By contrast, in Figure 5.10(b), a distinctly different phase shift $\Phi_{s p p}$ occurs with a smoother, continuous variation when rotating the $\frac{\lambda}{4}$-plate between the limiting $p$ - and circularly polarized excitations. For the $\beta$ edge, the SPP phase is advanced or retarded when SAM matching favors or disfavors the SPP launching. The same, but inverted behavior is observed for the $\beta^{\prime}$ edge (Figure 5.10(b)). Such complicated phase variations can be explained by a superposition of the $\boldsymbol{P}_{l}$ and $\boldsymbol{P}_{t}$ in forming the SPP wave, as will be described in the next section. 


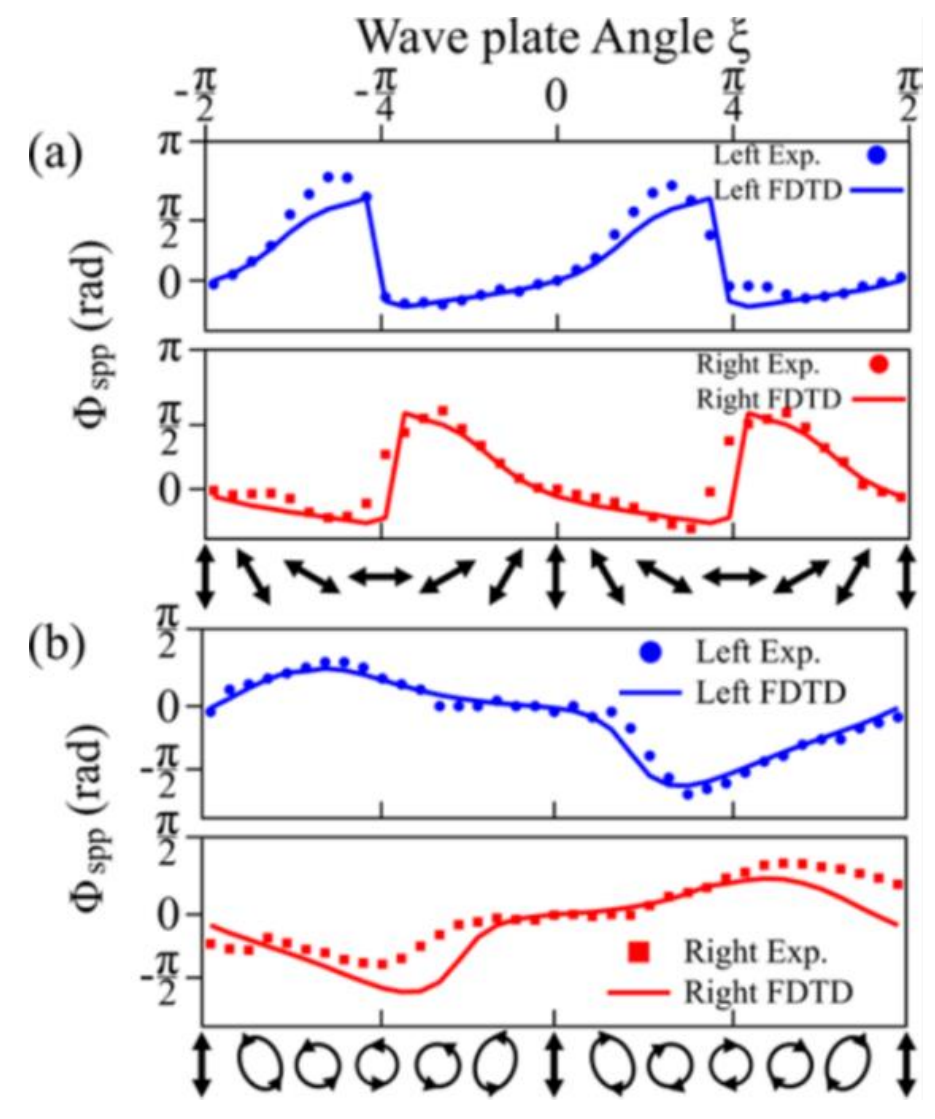

Figure 5. 10 Plot of the polarization dependent initial phase of SPPs launched from the $\beta$ and $\beta$ ' edges relative to the p-polarized excitation acquired on left (blue) and right (red) side of the Ag island. The $\xi$ angle is shown on the top axis, and the corresponding laser polarization at the bottom of each graph. (a) Initial SPPs phase, $\Phi_{s p p}$, for linearly polarized excitation, showing its variation with a period of $\xi=\frac{\pi}{2}$. The phase from $\beta$ edge changes slowly when $-\frac{\pi}{4}<\xi<0$, more rapidly for $0<\xi<\frac{\pi}{4}$, and has sudden jumps at $\pm \frac{\pi}{4}$. (b) $\Phi_{\text {spp }}$ with circular excitation a smoother variation for all polarizations occurs. When the in-plane SAM of light $\left(S_{\|}^{L}\right)$ and the transverse SAM of SPPs $\left(S_{\perp}^{s p p}\right)$ are parallel $\Phi_{s p p}$ is advanced $\left(\beta\right.$ edge at $\left.-\frac{\pi}{4}\right)$, and when they are antiparallel it is retarded. 


\subsubsection{Longitudinal and Transverse Coupling of SPPs}

The $\Phi_{s p p}$ observations in Figure 5.10 can be explained more concretely by considering the $\boldsymbol{P}_{t}$ and $\boldsymbol{P}_{l}$ components of the SPP field excited at the $\beta$ edge. In Figure 5.11(a), we plot the simulated spatial distribution $\boldsymbol{P}_{z}$, the z-component of the total polarization field, $\boldsymbol{P}_{\text {tot }}$, along the dashed line in Figure 5.3(b), for $\mathrm{t}=53.5 \mathrm{fs}(35 \times 2 \pi$ phase delay) after the pulse maximum (at $\mathrm{t}=$ $0 \mathrm{fs})$ has interacted with the $\beta$ edge, which is the distance origin. For p-polarization $(\xi=0)$, where $\boldsymbol{E}_{\perp}$ is maximum, clear beating is observed due to interference between the light and the SPP fields. By contrast, for s-polarization $\left(\xi=-\frac{\pi}{4}\right)$, when only $\boldsymbol{E}_{\|}$exists, the beating is nearly suppressed, because the interference can only occur through the in-plane superposition of $\boldsymbol{E}_{\|}$and $\boldsymbol{P}_{l}$, which makes a negligible contribution to the $2 \mathrm{PP}$ signal. For this reason, the $2 \mathrm{PP}$ signal with s-polarized excitation is essentially unmodulated. The same situation occurs for s-polarization at $\xi=\frac{\pi}{4}$, except that now the $\boldsymbol{E}_{\|}$field is $\pi$-phase shifted with respect to that at $\xi=-\frac{\pi}{4}$ and consequently, $\boldsymbol{P}_{z}$ is also $\pi$-phase shifted, but the $2 \mathrm{PP}$ signal is independent of its sign. 

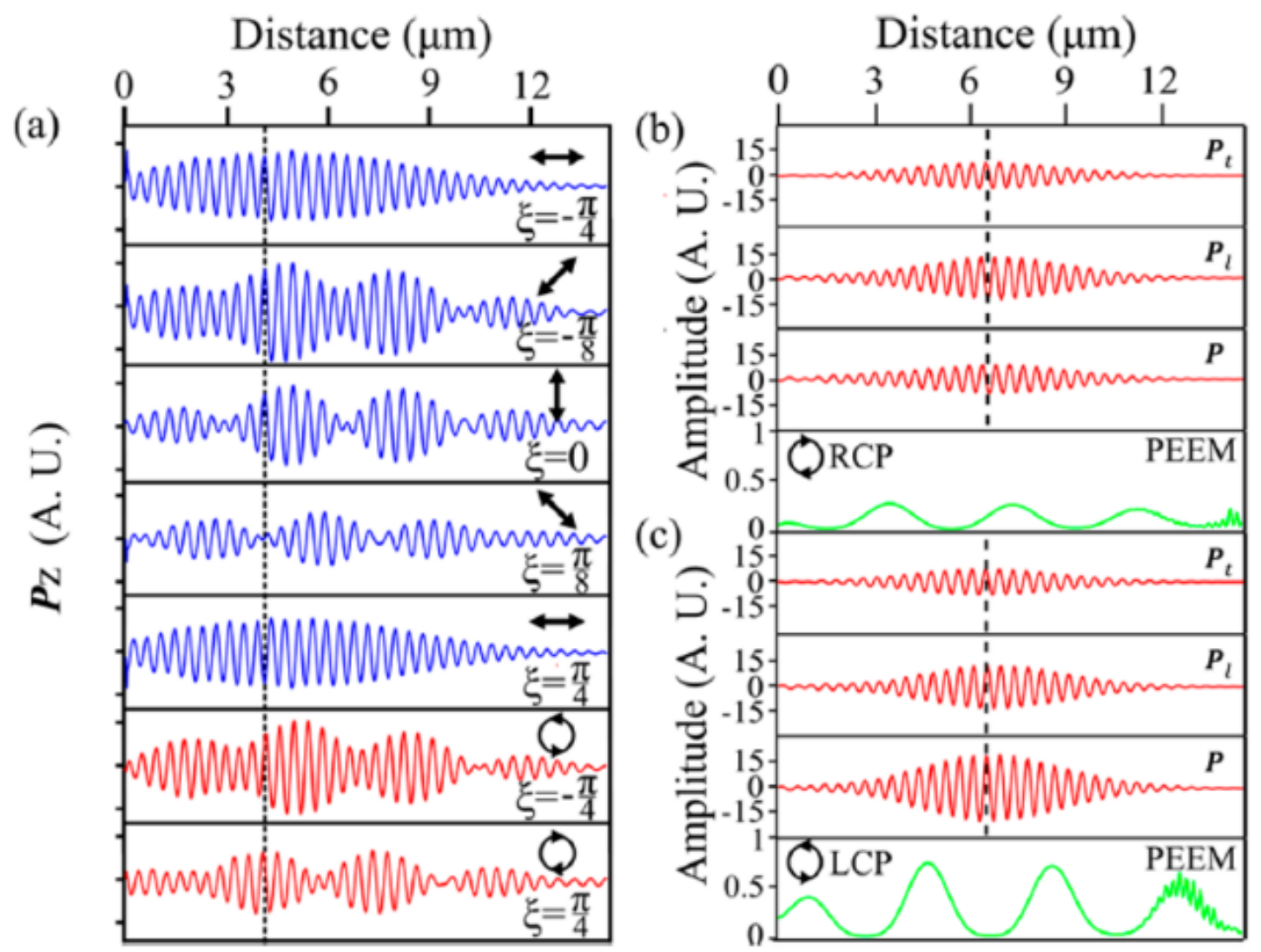

Figure 5. 11 (a) The z component of the simulated total polarization field $P_{z}=P_{t o t}(x, y, \tau) \cdot \widehat{n}_{\perp}$ taken $6 \mu \mathrm{m}$ away from island symmetry axis [dashed arrow in Fig. 1(d)] at $53.5 \mathrm{fs}$ after the pulse maximum interacted with the coupling edge for various linear (blue) and circular (red) polarization states of light. All of the polarization fields can be obtained by properly superimposing p- and s-polarized light based on the Jones transformation matrix for phase retarders. The intermediate polarizations show both amplitude and phase changes in agreement with experiment. (b, c) Profiles of $\boldsymbol{P}_{\boldsymbol{t}}$ and $\boldsymbol{P}_{l}$ components and their superimposed SPP field $P$, based on Huygens pricple simulations, at 53.5 fs acquired from the same positions as in the FDTD simulation. Clear amplitude asymmetry and phase change is observed in $P$ as well as the calculated PEEM profiles, for LCP and RCP excitation.

The spatial distribution $\boldsymbol{P}_{z}$ of other linear polarizations are simulated independently, but they can be interpreted by a superposition of the $\boldsymbol{P}_{z}$ fields at $\xi=0$ and $\xi=-\frac{\pi}{4}$ (p-and s-polarized 
excitation). A clear spatial shift of the beating envelope is seen, which represents the relative phase change between the SPPs and the incident light. Therefore, at the dashed line in Figure 5.11(a), the phase of the $\boldsymbol{P}_{z}$ is seen to change slowly when $-\frac{\pi}{4}<\xi<0$, because the contributing excitation and SPP fields are approximately in-phase, as seen for $\xi=-\frac{\pi}{8}$. But, the overall phase change is more rapid when $0<\xi<\frac{\pi}{4}$, because the fields are approximately out-of-phase, as seen for $\xi=\frac{\pi}{8}$. In both cases, the phase changes of $\boldsymbol{P}_{Z}$ is faster when $\boldsymbol{E}_{\perp}$ dominates because of its pronounced interference with $\boldsymbol{P}_{t}$, but the change is slower than when $\boldsymbol{E}_{\|}$dominates because its contribution to the $2 \mathrm{PP}$ signal is much smaller.

Qualitatively similar considerations apply to the rate of phase change for circularly polarized excitation; when the superposition is in-phase, the phase shift is slower, and vice versa. Characteristics of the circularly polarized excitation is that the phase of in-plane polarization is advanced (retarded) relative to p-polarization $(\xi=0)$ when the longitudinal components of $\boldsymbol{S}_{\|}^{L}$ and $\boldsymbol{S}_{\perp}^{\text {spp }}$ are parallel (anti-parallel). The phase shift, and in turn the shift of the beating pattern in PEEM, can also be interpreted by the interference between the SPP field evolved from $\boldsymbol{P}_{t}$ and $\boldsymbol{P}_{l}$, whose amplitudes and relative phases are determined by the incident light ellipticity.

To further show how the asymmetry is caused by the interference between SPPs launched by components of the external field in the case of circularly polarized excitation, we show results from an analytical simulation based on the Huygens-Fresnel principle,[71] where the coupling into $\boldsymbol{P}_{l}$ and $\boldsymbol{P}_{t}$ components of the generated SPP fields are calculated separately from their excitation fields $\boldsymbol{E}_{\|}$and $\boldsymbol{E}_{\perp}$. The components, as well as the total plasmon field $\boldsymbol{P}=\boldsymbol{P}_{l}+\boldsymbol{P}_{t}$ at the same time instant as in the FDTD simulation in Figure 5.11(a) for the LCP and RCP excitations, are plotted in Figure 5.11(b)(c). The green curves in Figure 5.11(b)(c) show the calculated line profiles of the 
PEEM signal, which give the beating patterns that remain after integration over time. The initial phases of the $\boldsymbol{P}_{l}$ and $\boldsymbol{P}_{t}$ fields are fitting parameters that can be extracted from the distance between the coupling edge and the first beating maximum, and account for the superposition of the SPP and the incident light fields. The phase of $\boldsymbol{E}_{\|}$is shifted by $\pm \frac{\pi}{2}$ with respect to $\boldsymbol{E}_{\perp}$ to account for the light ellipticity. The $\boldsymbol{P}$ field, shows the asymmetry in amplitude for the opposite ellipticities, resulting from the phase that is defined by superposition of the $\boldsymbol{P}_{l}$ and $\boldsymbol{P}_{t}$ components, where the dashed line at the center of the pulse marks the phase relationships that lead to the partial 2PP enhancement and suppression. The $\boldsymbol{P}$ further affects the PEEM line profiles by interfering with excitation field, which gives the line integrated CPL yield asymmetry of $=-0.31$. Lastly, besides an enhancement and suppression of the photoemission signal, the resultant phases of $\boldsymbol{P}$ for LCP and RCP are nearly $\pi$ shifted. Consequently, the beating maxima for LCP light are shifted farther from the coupling source with respect to RCP excitation. If examined from the $\beta^{\prime}$ edge, the roles of RCP and LCP light are reversed.

\subsection{Dynamics of Spin-Angular Momentum Coupled SPPs}

Beyond the spatial imaging of the plasmonic fields, we also performed an ITR-mPEEM experiment to image evolution of the spin-momentum locked SPPs in space and time. The movies are generated by scanning the delay between identical pump and probe pulse pairs with a MachZehnder interferometer in $\sim 100$ as steps.[76] After generation in the interferometer, the linearly polarized pulse-pairs passed through the $\lambda / 4$ retardation plate to generate either LCP or RCP light. Snapshots from the movies in Figure 5.12(a)-(d) show 2P-PEEM images excited by the RCP light 
from the experiment and Huygens-Fresnel simulation at delay $\Delta t$ of $0 \mathrm{fs}$ and $27.6 \mathrm{fs}$. When $\Delta t=0$ $\mathrm{fs}$, the photoemission signal is the same as insingle pulse 2P-PEEM images where the $2 \mathrm{PP}$ signal is not pronounced at the far side of the island because the generated SPP pulse has not had enough time to propagate there.[76] When the delay exceeds the pulse duration, the pump excited SPPs have propagated so a clear beating pattern with the probe pulse field is seen at the far end of the crystal.
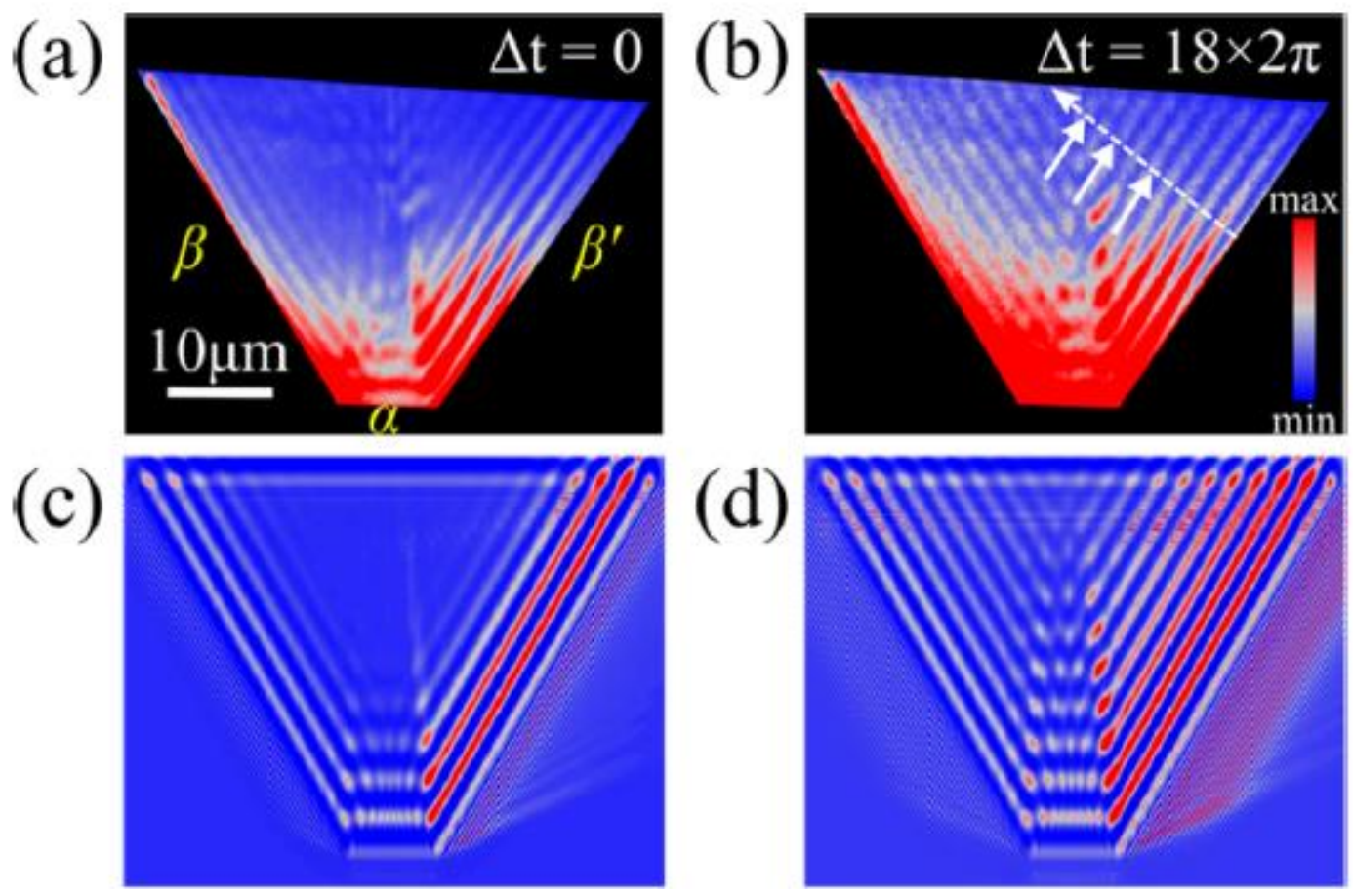

Figure 5. 12 (a)(b) Snapshots of experimental ITR-2PEEM images excited by RCP light at pulse delay time $\Delta t=0$ fs and $\Delta t=27.6 \mathrm{fs}$, respectively. $(\mathrm{c})(\mathrm{d})$ The corresponding snapshots of the simulated ITR-2PEEM images for the delays in (a) and (b) based on the Huygens-Fresnel model. At $\Delta t=0$ fs, distinct beating patterns are only visible near the island coupling edges. At $\Delta t=27.6$ fs, the beating patterns away from the edges become more pronounced due to the interference between the probe pulse and the pump excited SPP field. 
Additional information to be gained from ITR-mPEEM movies is the phase and group velocities of SPPs, and potentially their dephasing lengths.[77] We do not extract the dephasing lengths, because at $460 \mathrm{~nm}$ they exceed the dimensions of the Ag island. Figure 5.13(a) plots the displacement of the second beating maximum $D_{B}$ of spin-momentum locked SPPs as a function of pump-probe delay when launched from the $\beta$ and $\beta^{\prime}$ edges with opposite light helicities. The rate of maxima displacement, $\frac{D_{B}}{\Delta t}$, is given by the phase evolution of SPPs, and hence their phase velocity, $\boldsymbol{v}_{p}=\frac{\omega}{\boldsymbol{k}_{s p p}}$, which is obtained from $\frac{D_{B}}{\Delta t}=\frac{\omega}{\left|\boldsymbol{k}_{s p p}-\boldsymbol{k}_{L}\right|}$. By linear fitting, we find that $v_{p}$ is independent of the SAM of light, as is expected for a medium that is neither chiral nor gyrotropic.[76] The extracted SPPs phase velocity is $v_{p}=0.90 \pm 0.02 \mathrm{c}$ for all generation conditions, which is slightly slower than the expected value of $0.95 \mathrm{c}$ from the Drude parameterization of the dielectric function of Ag.[78] The slower velocity from experiment can be caused by the uncertainty in the Drude dielectric function of Ag as it approaches the surface plasmon resonance, which sets the angle $\varphi$ between $\boldsymbol{k}_{s p p}$ and $\boldsymbol{k}_{L}$ through the Snell's Law and is a part in the calculation of $v_{p}$.[78] 

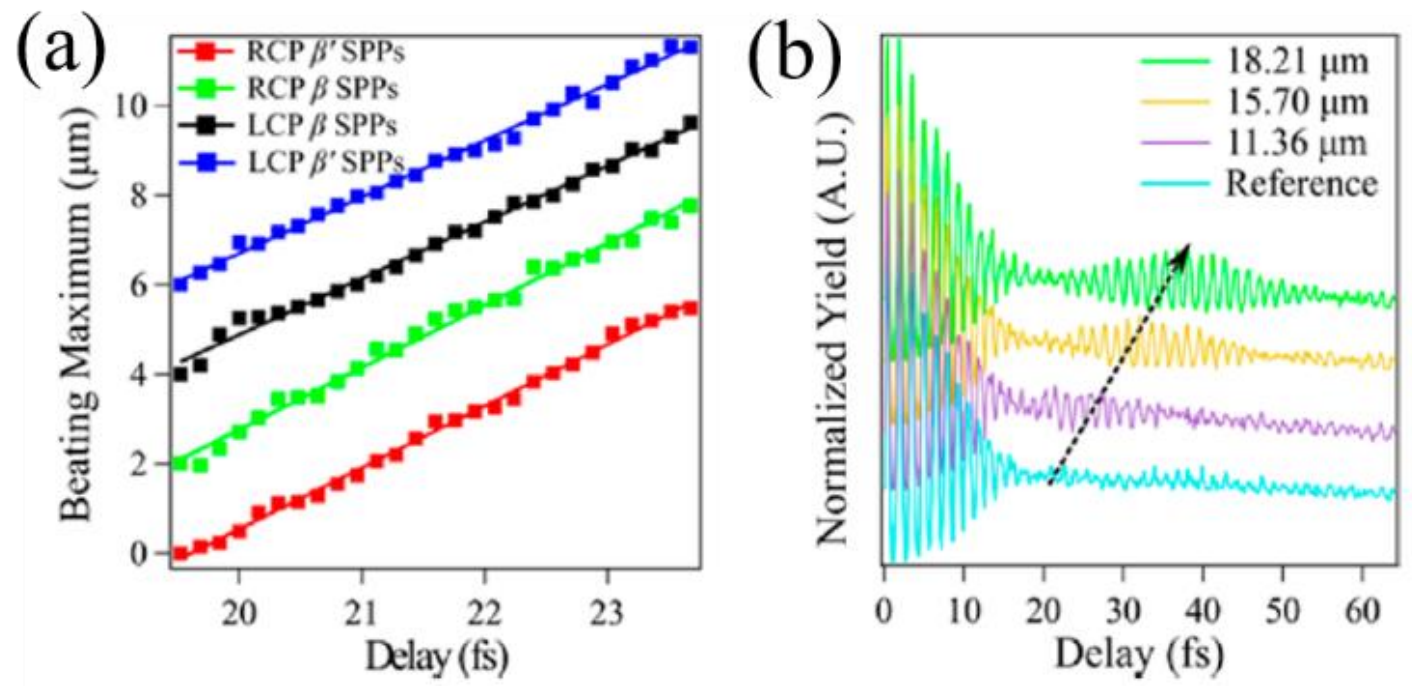

Figure 5.13 (a) Spatial displacement of the second SPPs beating maximum with respect to $\Delta t$ (The traces are displaced by $2 \mu \mathrm{m}$ along the vertical axis to enable visual comparison.). The four datasets correspond to SPPs launched from both the $\beta$ and $\beta^{\prime}$ edges for both the LCP and RCP light. The slopes observed from fitting the spatial displacements, which give the phase velocity of SPPs, are similar because the phase velocity of SPPs is independent of polarization. (b) Normalized I2PC traces taken at various points at the intersections of the dashed and solid arrows in 5.12(b) away from the $\beta^{\prime}$ edge. A two-pulse autocorrelation trace, taken at $\beta^{\prime}$ edge, is also plotted as a reference. A propagating interference envelope is observed in the tail of the I2PC trace when measuring $>10 \mu \mathrm{m}$ from the coupling edge. The envelope shift with $\Delta t$ gives the SPP group velocity.

The group velocity, $v_{g}$, which is defined by the wave packet motion, cannot be determined as precisely in PEEM images due to the finite dimension the Ag island. A different approach using the photoemission yield as a function of delay time, known as interferometric two-pulse correlation (I2PC) trice,[79] gives a direct way for evaluating the SPP wave packet motion. Figure 5.13(b) compares the I2PC traces by plotting the 2PP signal at various distances away from the coupling edge (white arrows in Figure 5.12(b)) in addition to an interferometric autocorrelation trace 
acquired at the edge $\beta^{\prime}$. Besides the pulse autocorrelation, the I2PC scans show a modulation that is caused by the delayed the probe pulse, which propagates to longer delays as indicated by the black arrow, for measurements with longer delay times. From the maximum of this propagating SPP pulse, we determine the vg of the spin-momentum locked SPPs for the variously polarized excitations to be $0.88 \pm 0.02 \mathrm{c}$, demonstrating that it is also polarization independent. The measured group velocity is consistent with previous 2P-PEEM measurements at $400 \mathrm{~nm}$.[78]

\subsection{Quantum Spin Hall Effect on Plasmonic Focusing}

In this section, I will describe the focus of SPP waves by a lens coupling structure. With $420 \mathrm{~nm}, p$-polarized light excitation, the SPP waves are focused into a spot of width $\sim 400 \mathrm{~nm}$. While with circularly polarized light, SAM dependent coupling in SPPs has an essential role on positioning the SPP focus, which is manifested in asymmetrical PEEM images. By numerical simulations, we show that the SPP fields at the focal point are chiral in the surface plane, which can be used for local selective excitation of chiral molecules.

\subsubsection{Symmetrical Focus of Plasmonic Lens}

A schematic of the experiment is shown in Figure 5.14(a). The sample consists of an electron beam evaporated $100 \mathrm{~nm}$ thick amorphous $\mathrm{Ag}$ film deposited on $\mathrm{Si}(001)$ substrate with natural oxidation layer at room temperature. Prior to Ag evaporation, $2 \mathrm{~nm}$ Ge film is deposited onto silicon as a transition layer to achieve better smoothness of the Ag film.[65] The Ge film improves the Ag film growth, but we do not detect any extraneous modification of the plasmonic 
properties of Ag films. A lens coupling structure is generated in the Ag film by focused ion beam milling (FIB); it has a semi-elliptical shape with the long axis in the excitation light direction (xaxis); its long and short axes are 8 and $4 \mu \mathrm{m}$, respectively.[69] The etching width and depth of the lens structure are 1 and $0.1 \mu \mathrm{m}$, respectively. The sample is transferred through air from fabrication to insertion into the AC-LEEM/PEEM instrument. Figure 5.14(b) shows a 1P-PEEM image of the lens coupler, with the spatial direction axes labeled .
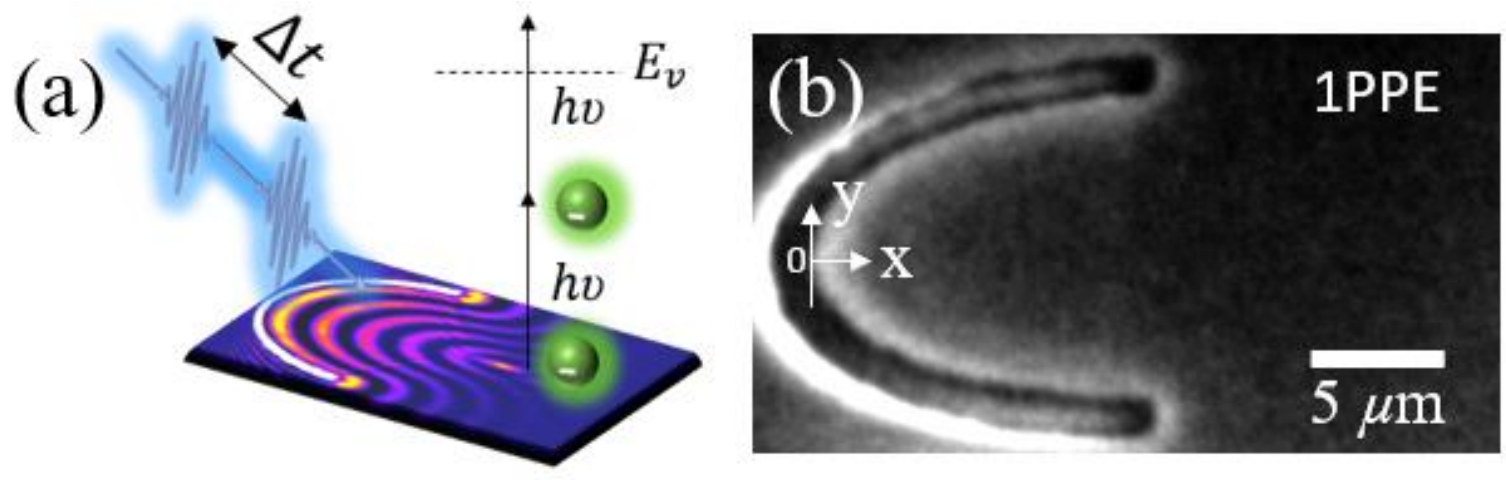

Figure 5. 14 (a) Schematic diagram of ITR-MP-PEEM excitation. $E_{v}$ denotes the vacuum level of Ag. (b) PEEM image excited with an $\mathrm{Hg}$ lamp (hv=4.9 eV) showing geometry of the plasmonic lens.

When illuminating the surface with femtosecond pulses, the sharp (subwavelength) edges of the coupling structure sample act as sources of momentum for coupling of light into SPPs. In Figure 5.15(a), we show the ITR-MP-PEEM images of the SPP interference when the lens structure is illuminated by $h v=2.95 \mathrm{eV}$, p-polarized light pulses. ITR-mP-PEEM measurements are performed as in other experiments.[50,56] In the case of Figure 5.14(a) and 5.14(b), the delay 
between the two pulses is 0 or $21 \times 2 \pi$ optical cycles $(\Delta \mathrm{t}=29.4 \mathrm{fs})$, respectively. For 0 fs delay, the PEEM images are the same as acquired by a single beam excitation; the PEEM image displays four beating fringes generated by beating between the optical and the SPP fields, which is prominent on the concave side of the lens coupler. The period of the interference along the $\mathrm{x}$-axis ( $\mathrm{y}=0$ ) is of $2.49 \mu \mathrm{m}$, which agrees with an estimated beating period of $2.45 \mu \mathrm{m}$, based on a calculation using the Drude dielectric function of Ag.[5] The corresponding FDTD simulation in Figure 5.14(c), reproduces the experiment well, except for having stronger intensity at the focal spot, which can be attributed to a faster attenuation of SPPs in the polycrystalline Ag film.
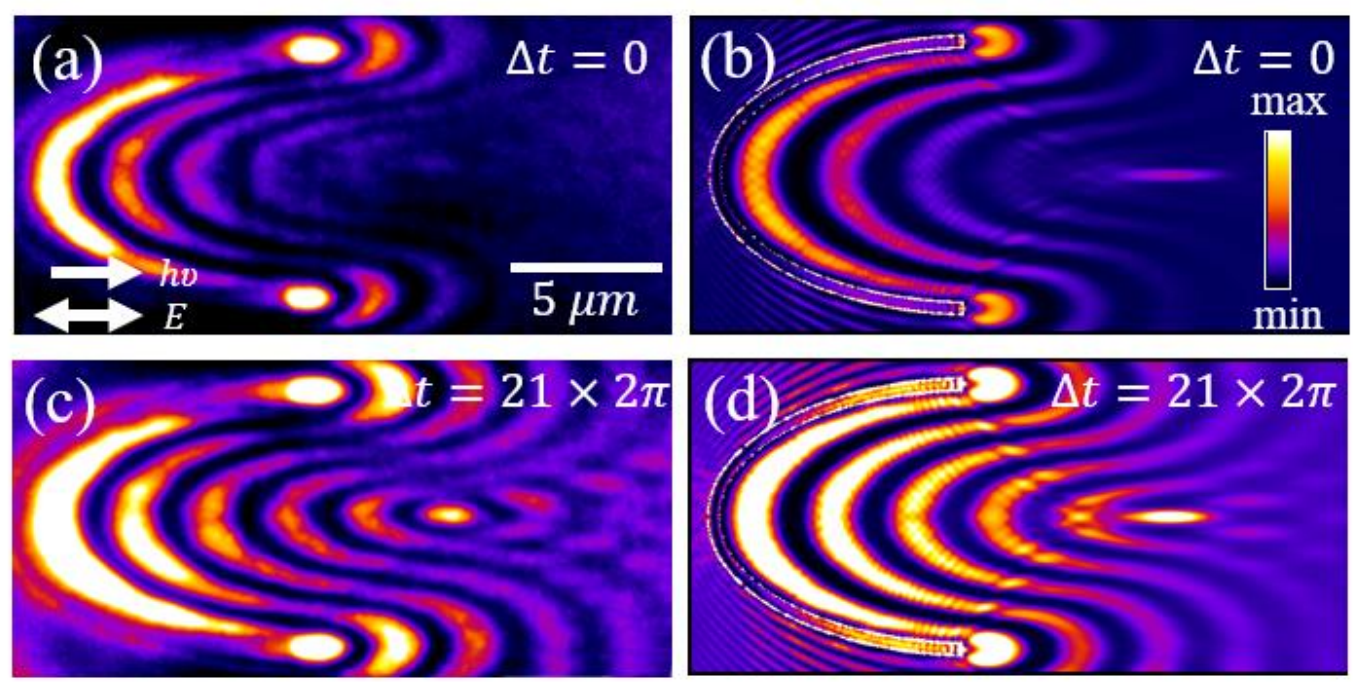

Figure 5. 15 (a)(b) Experimental and (c)(d) calculated time-resolved 2P-PEEM frames of the lens excited by p-polarized pulses at 0 and $29.4 \mathrm{fs}(21 \times 2 \pi$ radians $)$ pump-probe delay times. The signal enhancement away from the lens after the delay in (b) and (d) is caused by SPP focusing as its phase fronts propagate from the coupling edge. 
To visualize the time-dependent focusing of the SPP waves, we increase the pump and probe delay $\Delta \mathrm{t}$, by changing the length of one arm of the MZI, so that the probe beam interferes with the propagated SPP pulse that is generated by the pump beam.[30, 56] As a result, the total polarization field has an extra pump-probe beating envelope that appears in the PEEM images and propagates away from the lens as $\Delta \mathrm{t}$ is increased. In Figure 5.14(b)(d), we show the experimental and simulated ITR-PEEM images acquired at $\Delta \mathrm{t}=29.4 \mathrm{fs}$ corresponding to 21 optical cycles (at the center frequency) delay between the pump and probe pulses. At this delay we obtain the maximum signal from a distinct focal point of the plasmonic lens $\sim 12.5 \mu \mathrm{m}$ from its center edge, demonstrating its intended functionality. For the lens geometry, the width in the $y$-direction of the $E^{4}$ distribution (focus) is $\sim 400 \mathrm{~nm}$, i.e., it is comparable to $\lambda_{L}$.

\subsubsection{SAM Dependent Focusing}

When using an elliptically polarized light to excite, focus, and image SPP fields, the lens structure produces images with a distinct handedness that is imparted by the light polarization. In Figure 5.16, we show both the experimental and simulated PEEM images at delay times $\Delta \mathrm{t}=0$ and $29.4 \mathrm{fs}$, for the LCP and RCP excitation. At $\Delta \mathrm{t}=0 \mathrm{fs}$, the PEEM images have distinctly asymmetric beating patterns that reverse with respect to the optical plane upon switching the ellopticity. For the LCP light, the SPPs are generated preferentially generated from the $+y$ edge of the coupling structure and propagate towards the $-y$ direction, and for the RCP light the opposite is observed. Moreover, the LCP (RCP) focus is slightly above (bellow) the optical plane. Such asymmetries are a consequence of the plasmonic spin-Hall effect.[80] It is notable that imaging such SAM dependent shifts in light focusing is immediately detectable in PEEM imaging, whereas in other 
experiments it has required special plasmonic coupling structures, precise far-field imaging, or weak measurement techniques.[27, 80-82]
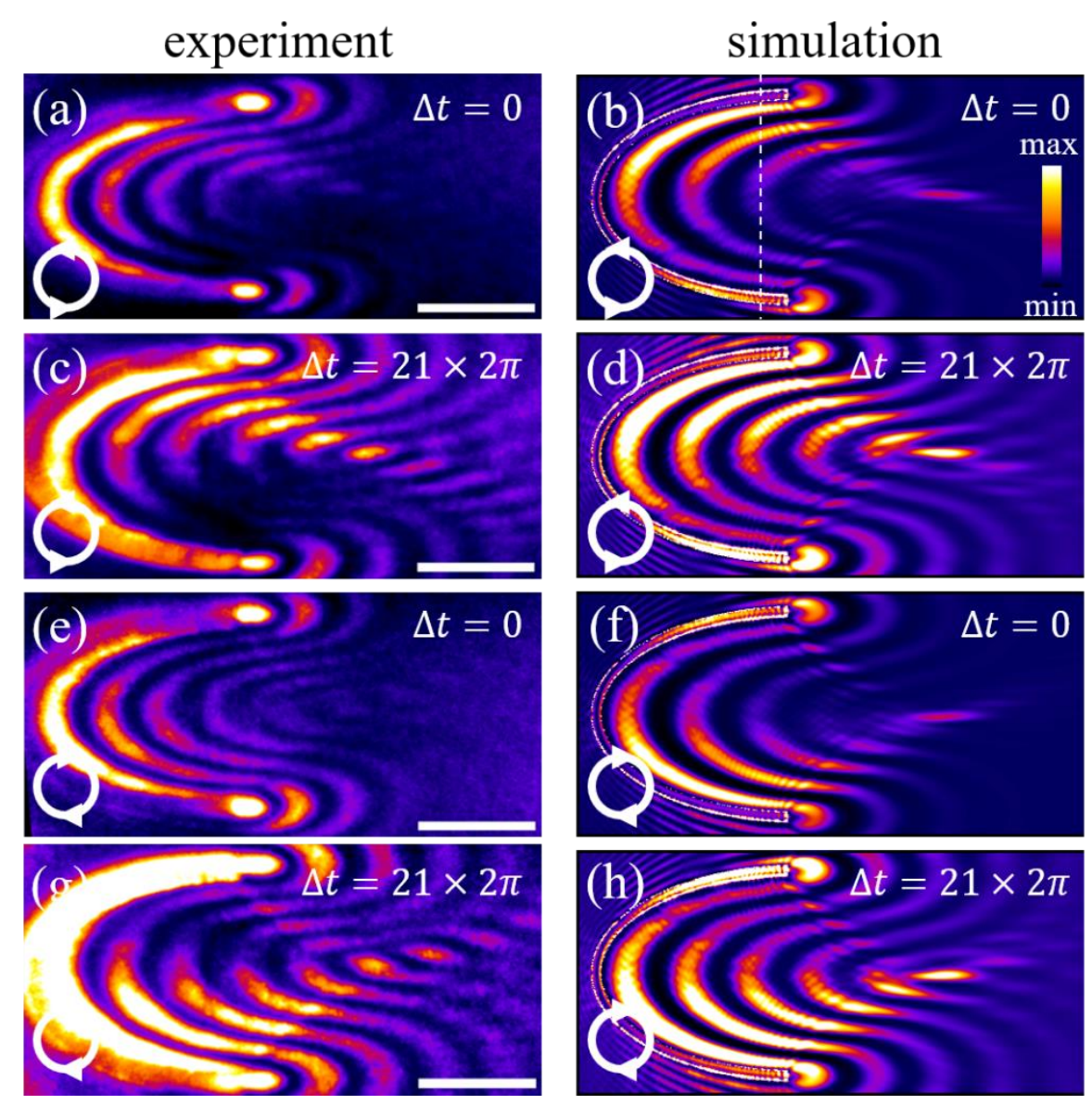

Figure 5. 16 Experimental (left) and calculated (right) ITR-MP-PEEM images of the plasmonic lens excitation by (a-d) LCP and (e-h) RCP light. The SPP waves travel and focus asymmetrically at $\sim 12.5 \mu \mathrm{m}$ in front of the lens. The distinctly asymmetric interference patterns for excitation with the opposite ellipticity manifest the spin-Hall effect. 
The physical reason for the position selective coupling of light into SPPs and ellipticity dependent focusing can be attributed to the SAM matching between the vacuum light and SPP modes it excites. When the SAM of light is in the direction of the SAM of SPPs, the excitation will be enhanced and when they are opposite it will be suppressed.[19, 80] Specifically, the SAM of LCP light [Figure 5.16(a)] projected onto the Ag surface points into the $-x$ direction and that of the RCP light points to the $+x$ direction. In the case of lens coupler, SPPs that are generated at the $+y$ edge and propagate to the focal point, have SAM with a component in the $-x$ direction. By contrast, SPPs propagating from the $-y$ edge to the focus have a SAM component in the $+x$ direction. Therefore, LCP light will preferentially excite SPPs from the $+y$ edge, and RCP light will excite preferentially them from the $-y$ edge. In Figure 5.16, for $\Delta \mathrm{t}=29.4 \mathrm{fs}$, the asymmetrically excited SPPs pulses are detected by the probe beam, showing an extended interference pattern with the same asymmetry as for the $\Delta \mathrm{t}=0 \mathrm{fs}$ excitation. Therefore, information on one side of the lens is dominantly transported to the focal point, while the information on the other side is nearly suppressed; this defines the effect light ellipticity on function of the lens coupling structure.

We note that SPPs, being evanescent fields, must carry a transverse SAM, $[14,15]$ which is orthogonal to the propagation vector $k$, and points in direction of rotation of the real into imaginary momentum of SPP according to the right hand rule.[13] In other words, SPPs are intrinsically chiral with the SAM of fields with the opposite wave vector $k$ pointing in the opposite direction. Although this aspect may contribute to the chirality density, which we define and discuss below, it is determined by the local electromagnetic fields that are created by the coupling structure. This chirality of SPP fields is referred to as the photonic quantum spin-Hall effect, in analogy to the electron quantum spin-Hall effect. In the former case, however, it is purely a 
classical effect, and "quantum" is an accepted misnomer, [80, 82] though one can argue that it is a manifestation of quantum and relativistic aspects of photons.[12]

An equivalent explanation of the observed asymmetry can be obtained by considering how different components of the light field excite SPPs. For the oblique excitation, the local electric field at the coupling structure has both an in-plane $(x-y)$ and an out-of-plane $(z)$ components, which are converted into and an SPP field by driving its longitudinal and transverse components. When launching SPPs with p-polarized light, most of the excitation is through driving of the transverse component of SPPs by the $z$-component of incident field. The longitudinal component of SPPs, however, is driven by the in-plane component of the external field. In Figure 5.17(a), we show a calculation of the $z$ component of the total polarization field, $P_{z}, 30$ fs after the optical pulse maximum has interacted with the coupling edge, excited by the $p-, s^{-}$, and circularly polarized light; the calculation is performed along the $y$ direction at the dashed line (at $x \sim 6.5 \mu \mathrm{m}$ ) that is indicated in Figure 5.16(b). For $p$-polarized excitation, $P_{z}$ is symmetric with respect to the lens symmetry axis $(y=0)$, because the $z$-component of the incident field is also symmetric and dominates the SPP excitation by transverse coupling. Although, $p$-polarization also has an in-plane field component parallel to the $x$-axis, its excitation of the longitudinal component of SPPs is also symmetric for the coupling structure, but its contribution to the $2 \mathrm{PP}$ signal is expected to be much weaker than the out-of-plane field excitation. For $s$-polarized excitation along the $y$-direction, however, the $P_{z}$ distribution in Figure 5.17(a) is anti-symmetric, because it is exclusively from the SPP field, which is excited by the in-plane incident $E_{y}$ field, and interacts with the coupling structure with a $\pi$-phase shift at the opposite edges. In addition, we see that the polarization fields at $y \sim-3 \mu \mathrm{m}$ in Figure 5.17 are almost in-phase for $p$ - and s-polarized excitations, but are out-ofphase, at $y=+3 \mu \mathrm{m}$. This is a vivid illustration of a phase difference between the longitudinally and 
transversely excited SPPs, which is dependent both on the polarization of vacuum light and the coupling geometry. Because circularly polarized light is a superposition of $p$-and $s$-polarized light, the relative phase between the transversely and longitudinally coupled SPPs from the $p$ - and $s$ components determines the enhancement and suppression of the total polarization field by their constructive and destructive interferences. Thus, the net effect is an asymmetry in the interference pattern, as shown in the lower panel of Figure 5.17(a). In particular, for the RCP light, the interference pattern can be constructed by superimposing the two $P_{z}$ fields in the first panel in Figure 5.17(a). For $-y$ region, because the $P_{z}$ fields excited by $p$ - and $s$-polarized light interfere constructively, their net effect is to enhance the $P_{z}$ field at $-\mathrm{y}$, while for $+\mathrm{y}$ region, the net effect is the suppression of the $P_{z}$ field due to their destructive interference. Therefore, such constructive and destructive interference of $P_{z}$ between $p$ - and $s$-polarized light excitations creates the asymmetry in the $P_{z}$. 

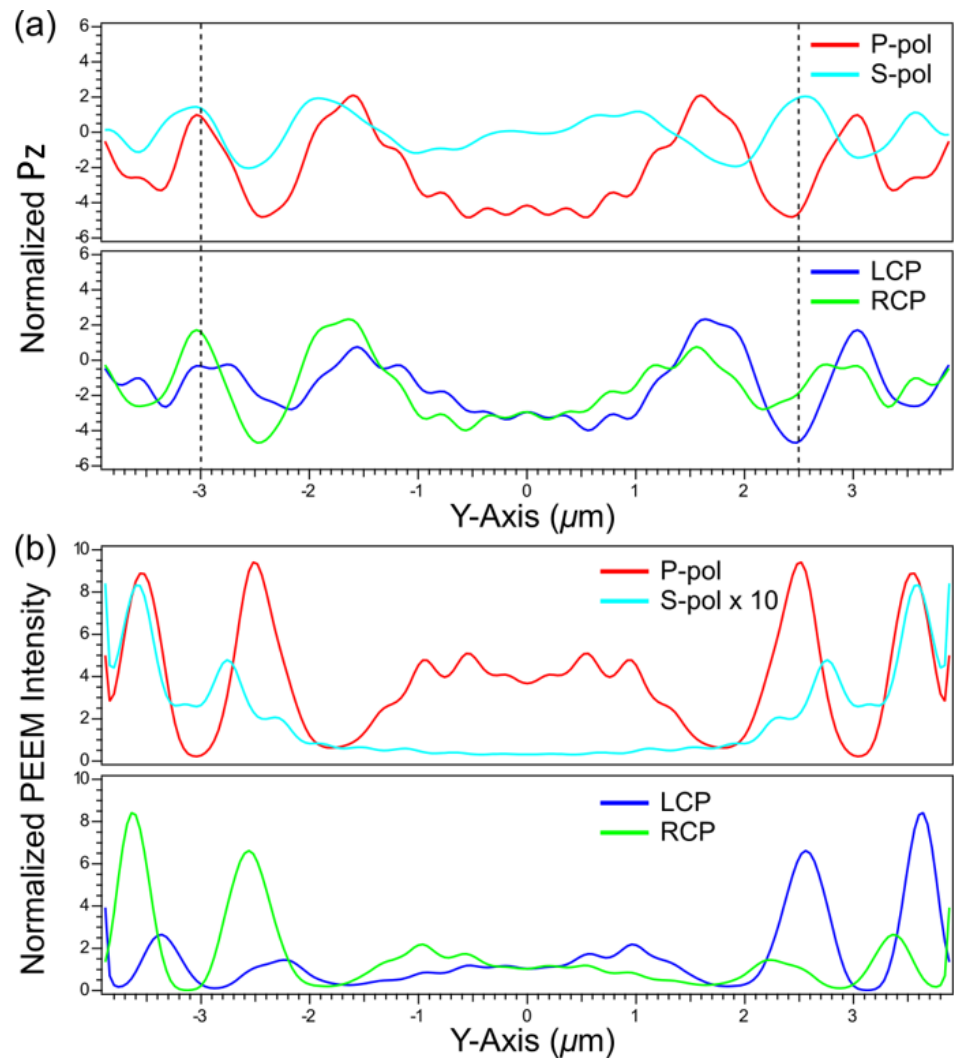

Figure 5.17 (a) The calculated z-component of the total polarization field taken at $x \sim 6.5 \mu \mathrm{m}$ for $\Delta \mathrm{t}=0$ [the dashed white line in Figure 5.16(b)] for selected polarizations. The period of the fast, weak oscillations is wavelength of the SPP field. For p-polarized light, a symmetric field distribution is excited because it is parallel and symmetric to the optical plane. For s-polarized light, the distribution is anti-symmetric, because its optical field is antisymmetric with respect to the optical plane creating a $\pi$-phase shift for coupling on the opposite sides of the lens structure. The field distribution for circularly polarized light has an asymmetry along $y$, which can be reconstructed by superimposing the $\mathrm{p}$ - and s- distributions with the appropriate amplitudes and phases. The dashed lines mark extrema in $P_{z}$ at $y \sim \pm 3 \mu \mathrm{m}$, showing the phase differences for all polarizations. (b) $I_{P E E M}$ line profiles integrated in time according to Eq. (1.22) at the same location as in (a). The time averaging washes out the asymmetry for the linearly polarized, but not the CPL light.

The asymmetry can be further visualized by the line profiles of PEEM intensity along $y$ direction in comparison with the $P_{z}$ fields, which are plotted in Figure 5.17(b). One can clearly see 
interference pattern with $\mathrm{p}$ - and s- polarized light, with the intensity about 10 times stronger in the case of p-polarized excitation. Less intense profile with s-polarized excitation is due to the lack of z-component in the incident field. In the lower panel of Figure 5.17(b), clear asymmetry is observed, which originates from the interference between the transversely launched SPPs by ppolarized light and longitudinally launched SPPs by s-polarized light as seen in Figure 5.17(a).

\subsection{Focus of Chirality of SPP Field}

\subsubsection{Field Chirality at the Focal Point}

Another feature that is imparted by superposition of transversely and longitudinally launched SPPs, is that the amplitudes and phases of the SPP fields are position dependent. Therefore, besides the intrinsic chirality of the SPP fields, the SPP fields in the $x-y$ plane are in general elliptically polarized due to superpositions from different sources on the converging lens

curvature. In Figure 5.18(a), we illustrate the mechanism for imposition of this in-plane chirality. Based on the Huygens principle, the lens coupling structure can be represented as an array of point sources, as has been demonstrated experimentally, with each point acting as a source of SPP fields.[69] The plasmonic lens is designed such that all the rays from a plane wave in vacuum arrive at the lens focus simultaneously regardless of time differences in interaction with the lens or the propagation lengths (times) as SPP fields.[69] Considering a specific point $P$ at positive $y$ above the focal point $F$, the total SPP field emanating from different points will converge with a range of position dependent phases and amplitudes. Therefore, the instantaneous in-plane direction of the electric fields is determined by the superposition of all the field components, which in 
general will produce an elliptical $x$-y field distribution. In Figure 5.18(b), we show the temporal profiles of SPP fields for the selected waves in Figure 5.18(a). One can clearly see that $k_{1}$ precedes $k_{2}$ and $k_{3}$ and has a larger amplitude because its propagation to point $P$ at positive $y$ is shorter. Therefore, the total field will first be dominated by $k_{1}$, with $k_{2}$ and $k_{3}$ being retarded. This will cause a counterclockwise (CCW) circulation of the elliptically polarized field, and by symmetry, the fields at $-y$ will circulate oppositely in a clockwise (CW) manner. As $P$ is scanned away from the lens optical axis, the relative phases and amplitudes defining the $x-y$ field will change varying the position dependent chirality.

In Figure 5.18(c)-(e), we show 2D maps of the z-components of the SPP field, superimposed with their in-plane elliptical fields that define the chirality at each point. For ppolarized light, distinct and symmetric SPP wave focus is observed, along with CCW chiral fields above, and CW chiral fields below the focal point. The red CCW polarization in (c) is a consequence of the superposition of fields from Figure 5.18(b). Moreover, no in-plane chirality exists along the focus $(y=0)$, which indicates the electric field oscillations are purely $x$ and $z$ polarized. Such linear polarization is a topologically protected polarization state, which arises by the destructive interference of $y$ polarized fields by the counter propagating component waves, i.e. fields of opposite in-plane polarization get canceled, creating a so-called L-line optical singularity.[83] For LCP excitation, because of the asymmetry in excitation in the $+y$ and $-y$ regions, the SPP fields are focused at $y \sim+150 \mathrm{~nm}$, thus the L-line singularity and $E_{z}$ field maximum (focus) are displaced upward. Consequently, along $y=0$, the in-plane field chirality is $\mathrm{CW}$. For the RCP light, the in-plane fields behavior is opposite because of the polarization of the incident light and its coupling into SPPs is simply reversed. 


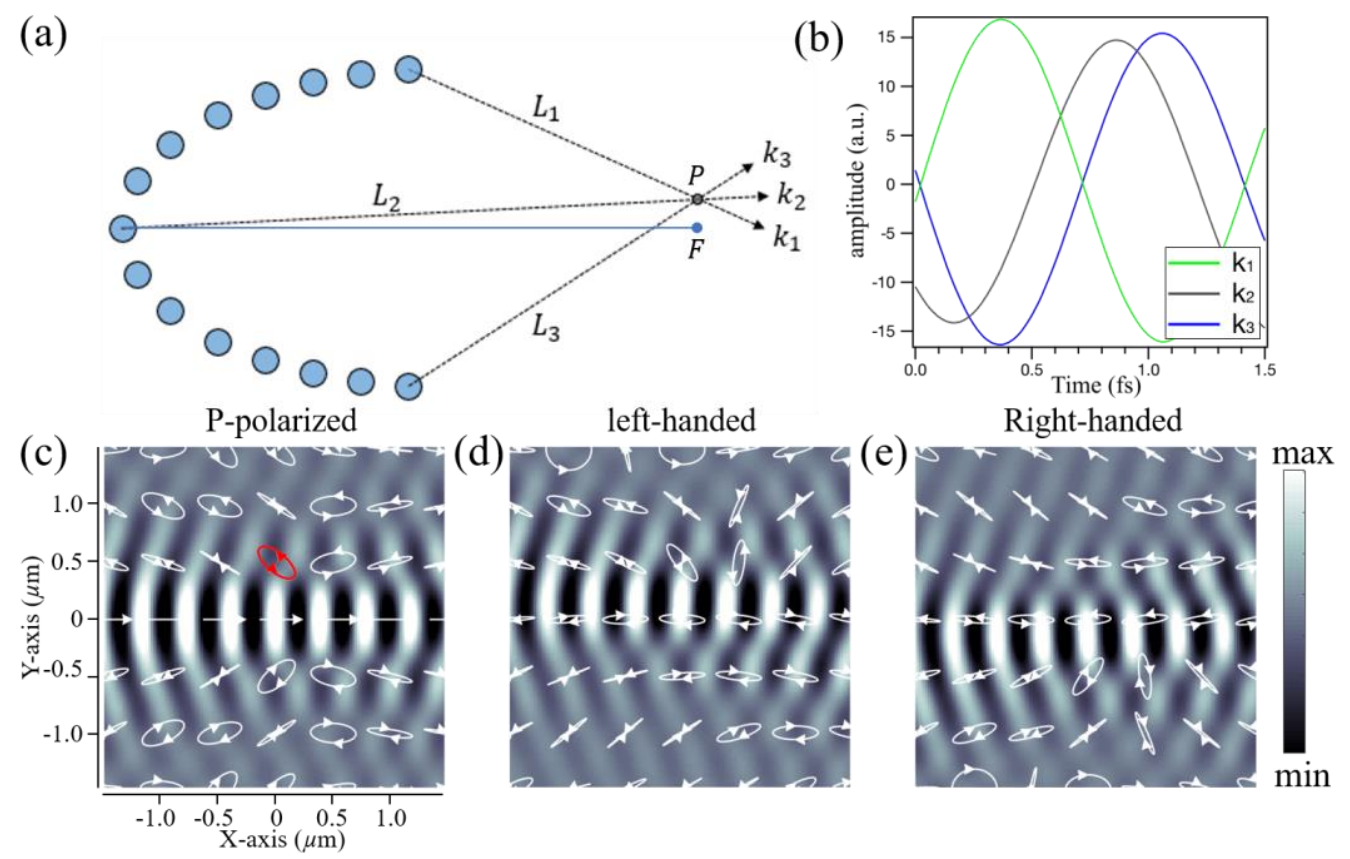

Figure 5. 18 (a) Schematic of the plasmonic lens based on Huygens principle. SPP waves from three selected origins are considered (right-most and left-most points) propagating with $k_{1}, k_{2}$ and $k_{3}$ vectors over distances labeled as $L_{1}, L_{2}$ and $L_{3}$ respectively. The point of consideration is labeled by $P$, which is above the focal point $F$. (b) The simulated $E_{z}$ field at $500 \mathrm{~nm}$ above the focal point, from the waves in (a). Meeting at $P$, the $k_{1}$ field precedes the others, causing the total field to circulate, which makes it chiral. (c)-(d) colormaps of the $E_{z}$ component of SPP fields coming to the focus, superimposed with the in-plane polarization states. The polarization states form L-line singularity on the optical axis of SPP wave excited with p-polarized light, but above and below the L-line they circulate counterclockwise, and clockwise. The red polarization ellipse is formed by the field profiles in (b). For LCP and RCP excitation the focus and the field ellipticity shifts up and down. 


\subsubsection{Chirality Density at the Focal Point}

Figure 5.18 shows that the in-plane fields generated by the lens coupling structure are locally chiral depending on the polarization of the excitation light. We therefore quantify the position dependent chirality of the fields by calculating the local chirality density[84, 85$]$ at the surface. The chirality density is defined as,

$$
C=-\frac{g}{2} \operatorname{Im}\left(E^{*} \cdot H\right)
$$

where $\boldsymbol{E}^{*}$ is the complex conjugate of the local electric field and $\boldsymbol{H}$ is the magnetic field. The chirality density defines the relative strength of interaction of electromagnetic fields with chiral objects, such as molecules.[84, 86, 87] In addition, the chirality density may also be used in enhancing spin-valley excitations in 2D valleytronic materials.[88] Figure 5.19(a)-(c) show the normalized chirality density $\left(C_{\text {norm }}\right)$ maps at the metal surface that is created by the lens structure upon excitation with $\mathrm{p}$ - and circularly polarized light; to enable relative comparison, $C_{\text {norm }}$ is normalized by the magnitude of the chirality density in the case of CPL illumination on an isotropic Ag surface. The left panel shows the full chirality density of the total field at the surface that is generated by the lens coupling structure, while the right panel shows the expanded map of $C_{n o r m}$ only from the SPP field in the region of the focal point. The chirality density is dominantly associated with the in-plane chirality of the SPP field, because the z-component of the SPPs magnetic field is weak. In addition, when the in-plane fields evolve CCW within an optical cycle, the negative chirality density (blue) is observed, and for CW fields the positive chirality density (red) is observed. It is evident that, for p-polarized light, SPPs launched from opposite edges form an anti-symmetric image in terms of chirality density, because the in-plane SPP fields circulate in opposite manner, as shown in Figure 5.18(c). Consequently, when SPPs with the counter 
propagating $k$-vector in the $y$ direction meet at the optical axis $(y=0)$, they form an L-line through the destructive interference of $E_{y}$ components, and constructive interference of the $E_{x}$ and $E_{z}$ components of the SPPs; the opposite field circulations cancel the $E_{y}$ component, and thus the inplane chirality, while the $E_{x}$ and $E_{z}$ components attain a maximum because of their constructive interference. The pure SPP contribution to $C_{\text {norm }}$ in the right panel goes to zero, because the linearly polarized incident field does not contribute to the field circulation.
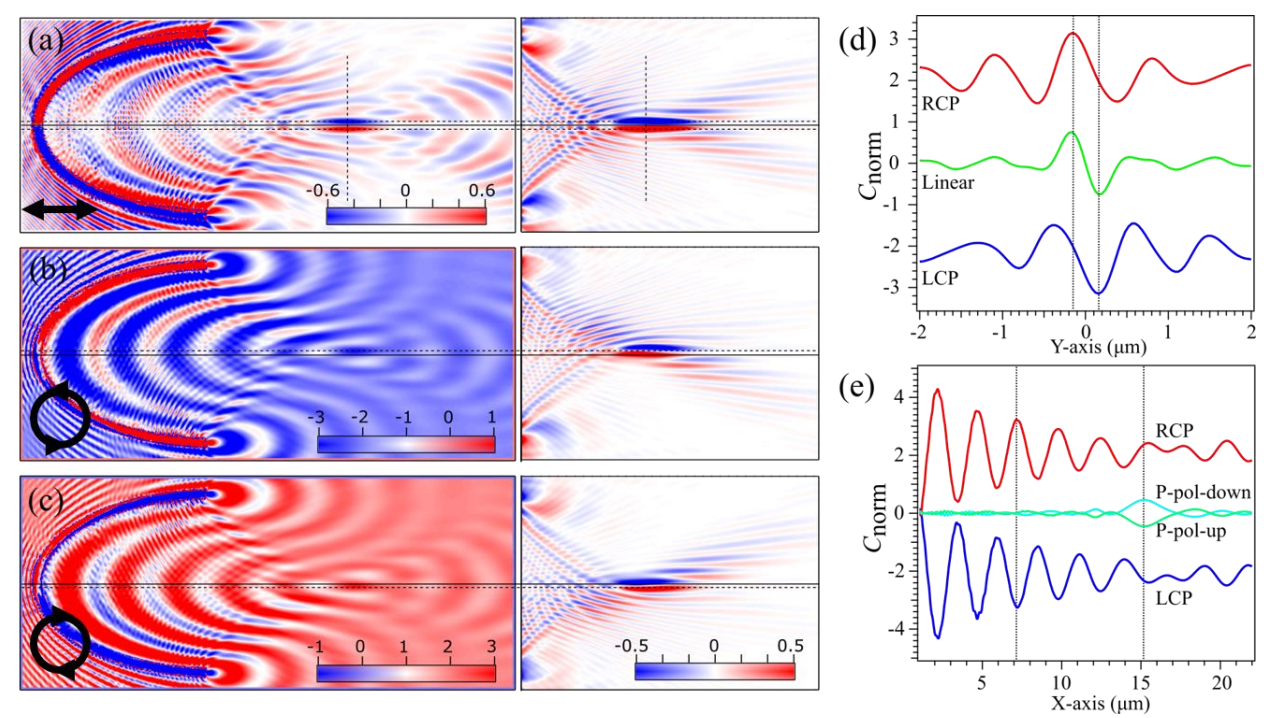

Figure 5. 19 (a)-(c) Calculated normalized chirality density $C_{\text {norm }}$ for p- and circularly polarized excitations.

The horizontal solid lines mark the lens optical axis $(y=0)$, and the dashed lines mark the maxima of the $C_{n o r m}$ focus. In the left panel, the total $C_{\text {norm }}$ includes the incident field, while the right panel shows chirality density due to SPPs only. (d)(e) are total chirality line profiles along the vertical (d) and horizontal (e) dashed lines in the left panels of (a)-(c). Clear enhancement is observed for CPL excitations. The extremum of the chirality near the focal point (guided by dashed lines) show that the chirality enhancement is only shifted in the $y$ direction dependent on the polarization of light. For p-polarized light, the chirality density inverts in the $y$ direction through the focal spot. For circularly polarized light, chirality is mostly dominated by the chirality of the incident light, with a clear focus following the SPP field. 
For the CPL excitations, as is evident in Figure 5.19(b)(c), the total chirality density maps are dominated by the chirality of the incident light, with a distinct asymmetry due to the SAM coupling to the SPP field. In addition, there is a clear focus of the chirality density, which is another evidence that the electric fields at the focal point are chiral in the $x-y$ plane. In the left (total) chirality density panel, depending on the incident light polarization, the focus and the local chirality density, are shifted up or down as marked by the dashed lines, which is consistent in the mapping of the polarization states in Figure 5.18(d)(e). In the pure SPPs panel, for the LCP, although the strongest chirality density is negative at $y \sim 150 \mathrm{~nm}$, the chirality density along $y=0$ is positive, which again agrees with the local polarization states of the SPP field. For the RCP, the chirality density is reversed.

Figure 5.19(d) plots the line profiles of the total chirality density along the vertical dashed line in Figure 5.19(a)-(c). One can see that the most enhanced region is localized around $y=0$. The extrema of the chirality density enhancement are shifted up and down for CPL because of the asymmetry in SPPs coupling. The $x$-axis line profiles of the total chirality are also plotted in Figure 5.19(e); the profiles are periodically modulated as expected from the observed beating patterns, and that the focal point under CPL excitation along $x$-direction lines up with the extrema as in the case of p-polarized excitation. Therefore, this shows that the plasmon lens has a fixed focal length along its paraxial axis, which is polarization independent. The external light polarization, however, induces a spatial shift of the total field focal point in the $y$-direction.

The simulations indicate that in addition to the spatial distribution of the focus of the chirality density, when SPPs are launched by CPL, the strength of the total chirality density at the focal point is enhanced by a factor of $~ 3.1$ compared to reflection of CPL from a continuous $\mathrm{Ag}$ surface. Therefore, chiral molecules placed at the focal spot will be excited with $\sim 9.6$ higher rate 
when experiencing the lens-induced field. Finally, the advantage of the enhanced chirality at the focal point is that, no additional nanoscale plasmonic particles are needed to enhance the rate of excitation. The SPP wave can propagate from several microns and focus on the flat metal surface, without interrupting the local geometry in the vicinity of chiral molecules environment. We note that the chiral density enhancement will vary with both the coupling structure, as well as the wavelength of light due to the fast plasmon dephasing. The calculated chirality density enhancements are for the generated coupling structure and excitation wavelength with no attempt to optimize it for a specific situation. 


\subsection{Plasmon Orbital Angular Momentum Generation}

In the previous PEEM experimental setup, the femtosecond light pulses come from an oblique angle, which launch and interfere with SPPs to form a beating pattern with a period that is larger than the wavelength of the SPP waves. While the advantage of oblique excitation it its strong photoemission signal because of the strong coupling of the out-of-plane light component, its drawback is that it creates a phase delay along the light propagation direction and lacks the SPP field information on the scale its wavelength. In this chapter, I describe the results where the SPP fields are generated from normally incident light, where light uniformly illuminates the sample with homogenous phase, and a geometric phase can be introduced by the SPP coupling structure. Under this excitation condition, we can study novel SPP phenomena by fabricating devices such as circular SPPs focusing lens or Archimedean spirals structures, where the SPP waves have engineered phase fronts to form desired spatio-temporal distributions. One particular case is the generation of surface plasmon polariton vortex, where the SPP fields undergo an orbiting motion around the core of the Archimedean spiral (AS), with geometrically engineered orbital angular momentum (OAM). When the SPPs vortex OAM=1, we generate a novel quasiparticle composed of the spin texture of the SPP field, which we define as plasmonic spin Skyrmion. Such quasiparticle has 3D spin texture that resembles the topological spin texture of stable magnetic Skyrmions in magnetic materials, but in the case of plasmonic generation, it lasts only for approximately the laser pulse duration. 


\subsection{SPP Vortex Generation by Archimedean Spirals}

Vortices are quantized angular momentum structures that appear in condensed matter phases such as superfluids and superconductors, with associated quantized flux. An optical analogue, i.e. optical vortices, can be generated by means of geometric phase front engineering, and more generally by speckle light scattering; in an optical vortex, the orbital angular momentum (OAM) of light has a tailored phase front, with order determined by the number of $2 \pi$ phase slips it accumulates during propagation.[89, 90] While conventional optics considers fields in three dimensions, an SPPs vortex can be generated and confined at a 2D metal/dielectric interface, with a strong field enhancement located at the vortex ring.[91-95] In this section, an SPP vortex generation device and imaging are described, along with the associated Skyrmion spin texture.

\subsubsection{Sample Design and Preparation}

SPPs vortex can be viewed as 2D propagating wave front, which creates a field distribution that orbits around a localized core, i.e. SPP phase circulates as waves propagate, with a phase singularity and vanishing out-of-plane SPP field at the vortex core.[91, 95] Therefore, to design a coupling structure than can generate such 2D SPP vortices, we must design structures that can launch SPPs so that their interference creates the phase singularity in real space. A simple way of generating a phase singularity is to have an accumulation of waves, where the phase orbits around a core. Such a structure is an Archimedean spiral, which can both focus the light to a central point and tailor the phase to locate the singularity at the SPP focus. In Figure 6.1, I show a schematic of an Archimedean spiral (AS) with a geometric charge $m=1$, where $m$ is a quantum number of OAM.

The geometric charge is defined by the number of $2 \pi$ phase accumulations along the spiral, which 
creates a phase singularity at the spiral center. The phase accumulation is determined by the opening $(D)$ after rotation by one cycle of the spiral termini, where $D=m \lambda_{s p p}$ and $m$ is an integer. The distance between the spiral center and each point on the spiral continuously varies, such that after one turn of the spiral, the phase of the SPP wave is delayed/advanced by $m \lambda_{s p p}$. Usual excitation optical fields in previous studies of SPP vortices have been circularly polarized, carrying SAM of $\sigma= \pm 1$, which couples with and is converted to the OAM at the plasmonic vortex to produce total OAM $L=m+\sigma$. Therefore, when an AS coupling structure with a geometric charge $m=1$, is illuminated with LCP light that carries $\sigma=-1$, the resulting SPP field is simply focused, because $L=0$, whereas for RCP light, i.e. $\sigma=1$, the resulting SPP field produces a vortex with $L=2 .[94,96]$

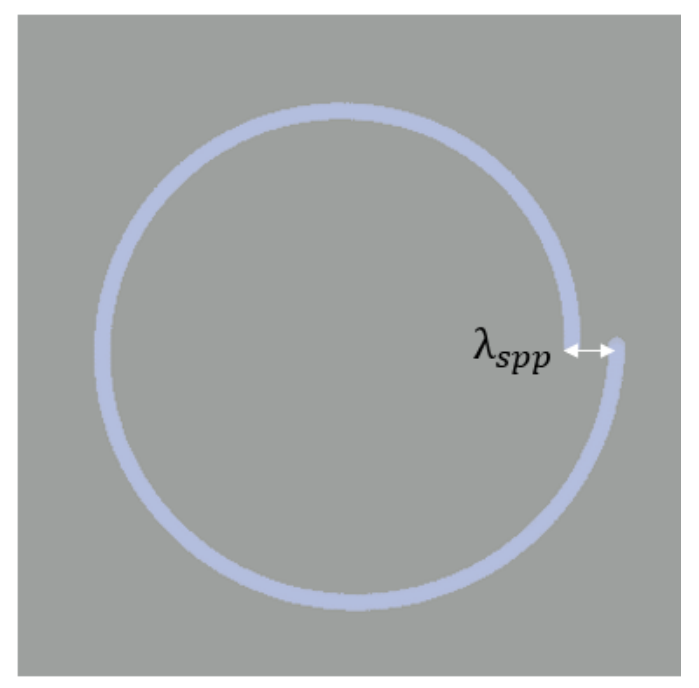

Figure 6. 1 Schematic of an Archimedean spiral of topological charge m=1.

The disadvantage of a design such as in Figure 6.1 is that if one needs to generate higher order OAM, one must have a large opening and SPP field amplitude decays rapidly as it propagates 
before it interferes to form a vortex. Thus, this will cause the field components to interfere partially causing them to penetrate into the vortex. Another type of design is to introduce multiple openings in an Archimedean spiral, each with an opening that totals $\lambda_{s p p}$. Then the SPP phase can accumulate multiple times due to the number of openings, and the distance to the center will be relatively constant. Therefore, the total OAM of the SPPs vortex will be determined by the number of openings of such spiral, or hereafter referred as the geometric charge of the spiral. A schematic of such design with $m=2$ is shown in Figure 6.2 (a). In my PEEM experiment, to have better coupling efficiency of SPP waves, I designed structures with concentric spirals, with spacing $\lambda_{\text {spp }}$, so that the SPPs generated at each spiral constructively interfere and propagate to the center. A schematic of such concentric spiral is shown in Figure 6.2(b).
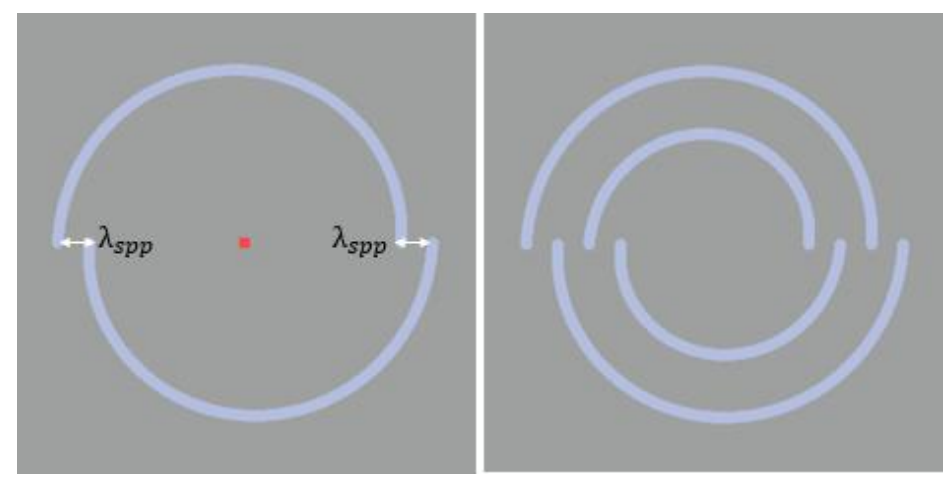

Figure 6. 2 Schematic of single slit and concentric slit, multiple opening, Archimedean spirals both with a topological charge $m=2$.

To experimentally prepare the AS coupling structures, an $\sim 100 \mathrm{~nm}$ thick Ag film is thermally evaporated onto $\mathrm{Si}(111)$ substrate with a natural oxide layer (in a Plassys electron beam 
evaporator MEB550S), and the AS structure is formed by focused ion beam milling (Seiko Instruments SMI3050SE FIB-SEM). The width of the slits is designed to be $100 \mathrm{~nm}$ using an ion beam current of $\sim 30 \mathrm{pA}$. Three turns of plasmonic AS structure with a spacing equal to the SPP wavelength are fabricated, so that the SPP waves from each turn add constructively. The radius of the AS coupling structure is chosen to be $19 \lambda_{\text {spp }}$, so that the optical excitation pulse no longer interferes with the SPP field when it forms a vortex. Figure 6.3 show several SEM images of AS structures with different geometric charges that I investigated.
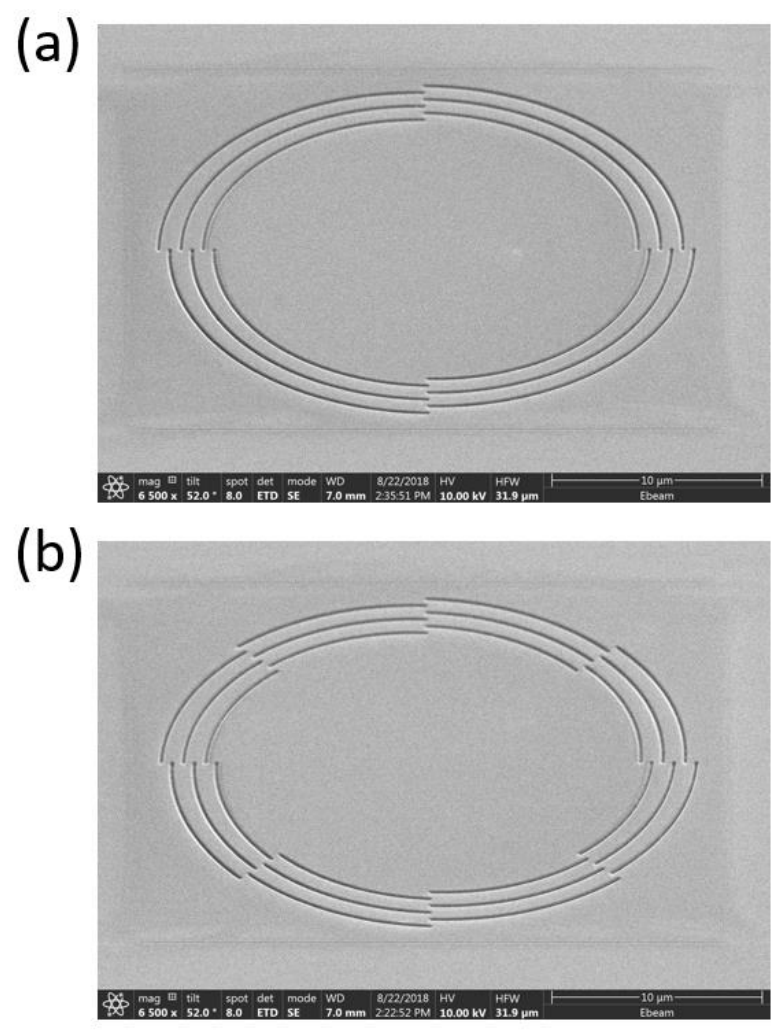

Figure 6. 3 SEM imagse of concentric multiple opening Archimedean spirals with topological charges $m=4$ (a) and $m=8$ (b). 


\subsubsection{Vortex Generation with Circularly Polarized Light}

To describe SPP vortices, in Figure 6.2 (b) I show simulated SPP fields generated by an $m=1$ Archimedean spiral, when excited with both the LCP and RCP light. In Figure 6.4 we show a series of simulated $z$-components of SPP vortices at the spiral center in quarter cycle increments. In the case of RCP excitation (Top panel), the vortex is composed of four rotating petals, with opposite signs (red, positive and blue, negative) of the transverse SPP fields. The four petals orbit around the core (phase singularity) with a period of an optical cycle. This orbiting motion of SPP field petals is a vortex with OAM $L=2$. In the case of LCP light $(\sigma=-1)$ in the lower panel, the angular momentum coupling gives $\mathrm{OAM} L=0$. Therefore, there is no phase singularity and the SPP fields come to a focus without the orbiting petal structure.
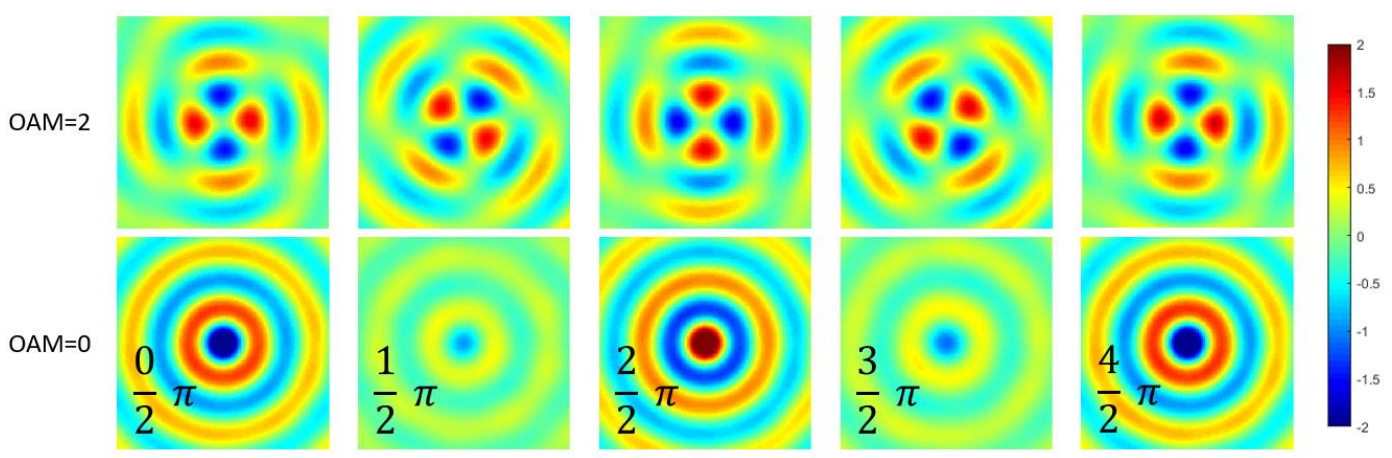

Figure 6. 4 Simulated evolution of the $z$-component of the SPP fields launched by excitation of an $m=1$ Archimedean spiral by RCP (top) and LCP (bottom) light. When the total OAM of the SPP field is $L=2$, four petals orbit around the vortex core, but when $L=0$, a plasmonic focus and no phase singularity occurs. 


\subsubsection{In-plane Component of SPPs Vortex}

So far, the SPP fields have been calculated by and FDTD simulation. They can also be calculated analytically, as will be shown in this section. This is helpful for describing the in-plane fields and the SAM that they generate.

Conventionally, plasmonic vortices refer to phase circulation of the out-of-plane $\left(E_{z}\right)$ component of the SPP fields, because the $E_{z}$ field has a stronger interaction with metals.[95] Here, I consider a simple circular coupling structure, which can be considered as an AS coupling structure with a zero geometric charge. Fig. 6.5 shows the coordinate system for describing the SPP fields considered in this section. According to the defined geometric parameters, one can obtain the spatial distribution of the $E_{z}$ at any point defined by the coordinates $R, \theta$, by integrating the SPP waves emanating from an infinitesimal increment at $(r, \emptyset)$ with respect to a continuous phase increment $\varnothing$, that is imparted by the circular polarization of light. RCP light with $\sigma=1$, generates the $E_{z}$ distribution corresponding to an $L=1$ SPPs vortex at an instant time shown in Fig. 6.6(a), with two major petals orbit around the core following the Poynting vectors indicated by the arrows.[95] For an SPP field having a wavelength $\lambda_{s p p}$, the diameter of the major ring is $R_{1} \sim 0.29 \lambda_{\text {spp }}$ 


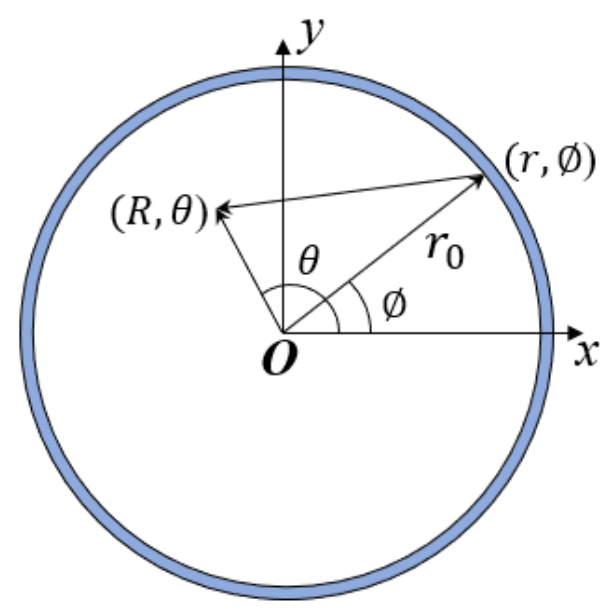

Figure 6. 5 Coordinate system of the circular plasmonic coupling strucutre.

Additionally, the in-plane SPP fields $\left(E_{\|}\right)$, which are often overlooked, but are important in my study, are also described. The spatial distribution of $E_{\|}$, which is $\frac{\pi}{2}$ phase shifted with respect to the $E_{z}$ field, differs significantly from the $E_{z}$ distribution. For an $L=1 \mathrm{SPP}$ vortex, the radial $\left(E_{r}\right)$ and tangential $\left(E_{t}\right)$ electric field can be obtained in a similar manner as the $E_{z}$ field, where the integration is performed with a vector projection of all SPP sources along the radial and tangential directions. The position dependent expression of the SPP vortex can be expressed as,

$$
\begin{gathered}
E_{r}(R, \theta) \sim\left[2 i R J_{1}\left(k_{s p p} R\right)+r\left(J_{0}\left(k_{s p p} R\right)-J_{2}\left(k_{s p p} R\right)\right)\right] \\
E_{t}(R, \theta) \sim\left[2 i R J_{1}\left(k_{s p p} R\right)-r\left(J_{0}\left(k_{s p p} R\right)+J_{2}\left(k_{s p p} R\right)\right)\right] \\
E_{z}(R, \theta) \sim i r J_{1}\left(k_{s p p} R\right)
\end{gathered}
$$

where the coordinates are defined in Fig. 6.5, and $J_{v}$ is the Bessel function of order $v$. The calculated spatial distributions of the $E_{r}$ and $E_{t}$ at the same time of Fig. 6.6(a) are shown in Fig. 6.6(b)(c). For $E_{r}$, there are two petals that tightly focused at the core, which orbit following the 
same Poynting vectors in Fig. 6.6(a). In addition, the field amplitude of $E_{r}$ reaches minimum around $R_{1}$. Finally, the $E_{t}$ component reaches the maximum at the vortex core, but its amplitude decays more slowly than $E_{r}$.
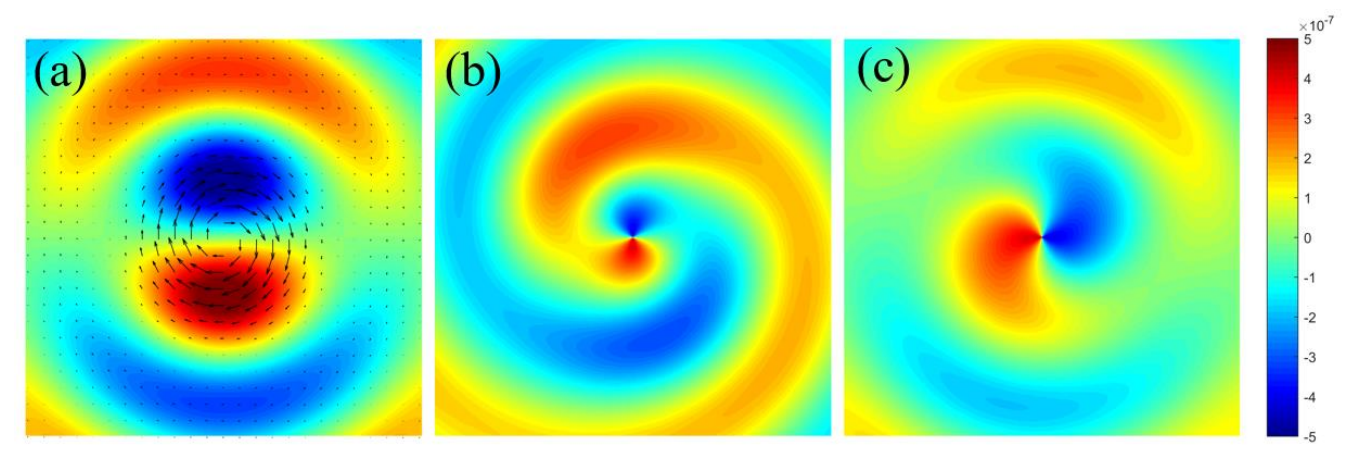

Figure 6. 6 Calculated field distributions of an $L=1$ plasmonic vortex. (a) $E_{z}$ field, (b) $E_{r}$ field and (c) $E_{t}$ field. The arrows in (a) indicate the Poynting vectors. The image size is $2 \lambda_{s p p}$.

\subsection{SPPs Vortex with Pure Geometric Charge}

\subsubsection{Field Evolution of SPPs Vortex Excited by Linearly Polarized Light}

The FDTD calculations reproduce the same fields as the analytical approach. Here I use the FDTD calculation to calculate that SAM distribution that is generated by the in-plane fields.

In addition to SPPs vortex generation with circularly polarized light, excitations using linearly polarized light at AS coupling structures have been reported to focus SPP waves, for AS 
structure with low geometric charge $m=1$ or 2 , where the effect of the phase engineering by the AS coupling structure is evident.[97, 98] In this section, I show both numerically and experimentally that AS coupling structure with $m=2$ excited by linearly polarized light generates a plasmon vortex that is purely dependent on the geometric charge, i.e. without the external SAM.
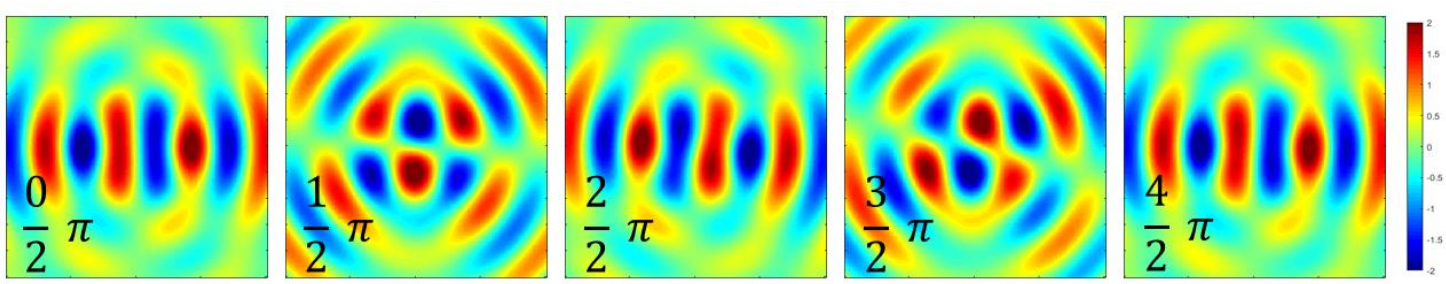

Figure 6. 7 Simulated evolution of the $z$-component of SPP field, launched at an $m=2$ Archimedean spiral by linearly polarized light.

Figure 6.7 shows evolution of the $z$-component of SPP field over one optical cycle, when launched from an $m=2$ Archimedean spiral by linearly polarized light. One can see that the field distribution repeats after one optical cycle in a clockwise rotation. The sense of rotation is determined solely by the geometric charge of the spiral device, because the linearly polarized light carries no SAM.

\subsubsection{Static PEEM Imaging of SPPs Vortex}

With the previous understanding of SPP vortices, I am interested in imaging the vortex dynamics dependent on the geometric charge that occurs in plasmonic focusing. I first show an 
image of the static 2PP pattern contributed by the field components in Figure 6.7. In this experiment the illumination is no longer oblique, but is reconfigured to occur at the surface normal. A schematic of the excitation setup is shown in Figure 6.8.

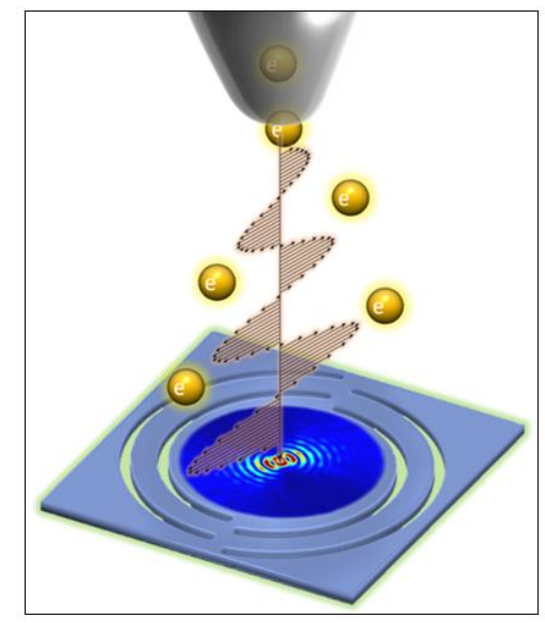

Figure 6. 8 Schematic of the experimental setup for SPP vortex generation.

A linearly polarized light incident in the the surface normal direction uniformly illuminates the entire spiral device to launch SPPs and form a plasmonic vortex. The static, i.e., integrated over single pulse excitation, image of the $2 \mathrm{PP}$ signal from the AS center is shown in Figure 6.9 (a). The excitation forms an $m=2$ vortex spiral, which has two strong photoemission spots, aligned in the $x$ - direction when excited linearly ( $y$-) polarized light. These two strong photoemission spots are attributed to the dominant $z$-component of the SPP fields, while the side fringes on the left and right sides are caused by a standing wave pattern of the SPP interference. In Figure 6.9 (b), I show the corresponding calculated time-averaged z-component of the SPP field based on equation 1.22, 
which resembles the experimentally observed photoemission pattern, thus confirming the origin of the photoemitted signal.
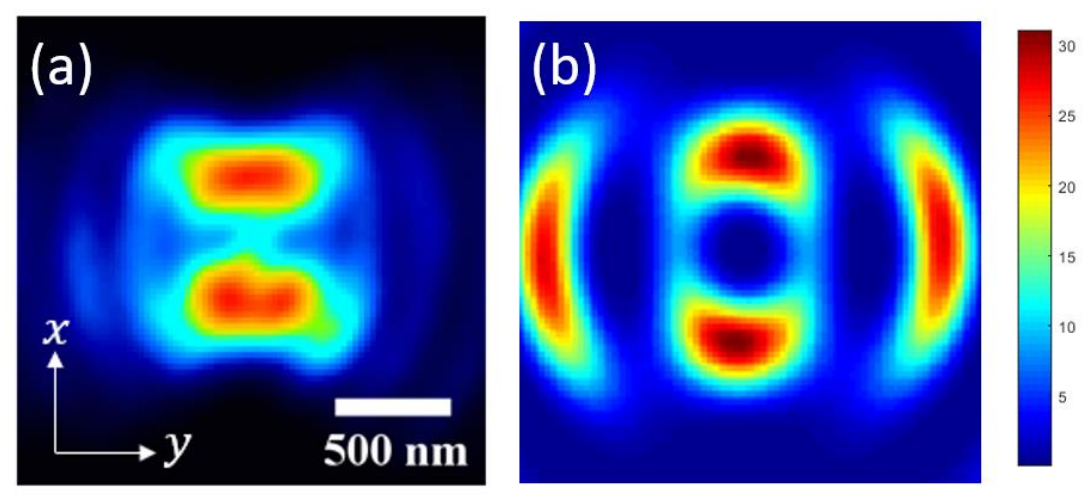

Figure 6. 9 (a) Experimental PEEM image of the SPPs vortex launched from $m=2$ Archimedean spiral with $550 \mathrm{~nm}$ linearly polarized light. (b) Simulated time-averaged $z$-component of the SPP fields.

\subsubsection{ITR-PEEM Imaging of SPPs Vortex Dynamics}

Just the static PEEM images do not confirm that the SPP fields indeed form a vortex. Therefore, I carry out time-resolved PEEM measurements, where the probe pulse detects the space and time evolution of SPP launched by the pump pulse. In Figure 6.10, I show a sequence of the pump-probe measurements corresponding to the image shown in Figure 6.9 (a), over a delay corresponding to one optical cycle. Initially the photoemission distribution resembles the static image such as in Figure 6.9 (a). As the probe pulse is advanced, the photoemission spots appear to rotate clockwise, which repeats after the delay is advanced by an optical cycle ( $2 \pi$ in phase). 

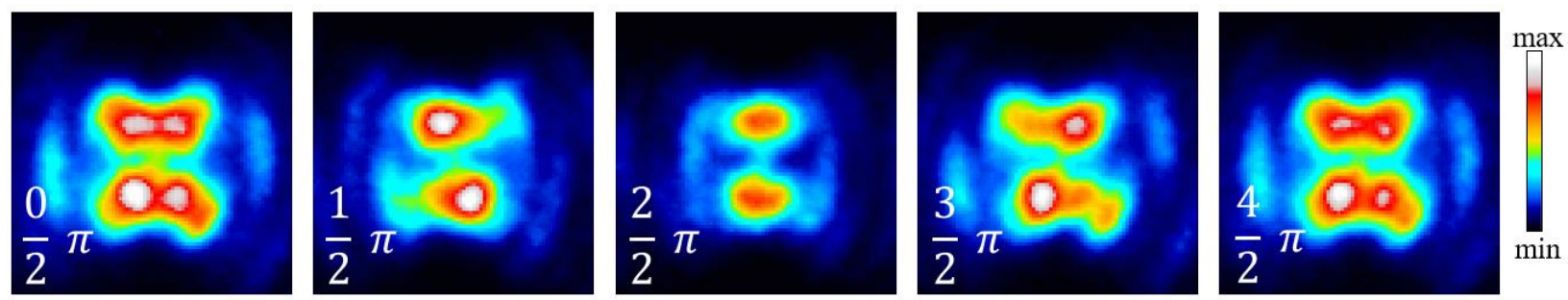

Figure 6. 10 Sequence of experimental time-resolved PEEM images within one cycle of light exitation.

For the normal incident light, the photoemission contrast in the time-resolved experiments can only occur through interference between the in-plane components of the SPP field and the incident light, because the incident light only contains the $y$-component. The $z$-component of the SPP fields, which contributes more strongly to the 2PP signal, however, only contributes a static (delay independent) background, because the external field does not interfere with it. Therefore, by demonstrating that the in-plane SPP fields rotate around the vortex core, we can confirm that we indeed generate a plasmonic vortex, even without introducing SAM of the external light.

\subsection{The Spin Texture of the Plasmonic Vortex}

\subsubsection{Plasmonic Spin Texture}

In this section, I first consider the simplest SPPs vortex with OAM $L=1$ (circular structure excited by RCP light, $m=0, \sigma=1$ ), and show that the SAM associated with such SPPs vortex has a topological texture that resembles that of a Néel type magnetic Skyrmion.[99] Then, in section 
6.3.2, I will show that similar Néel type SAM Skyrmion exists for SPP vortex formed by illuminating $m=2$ AS coupling structure with linearly polarized light $(\sigma=0)$.

When an SPP vortex is formed, the transverse spin of the SPPs, whose direction is locked transverse to their $k$-vector, must converge to or diverge from the vortex center, depending on its rotational direction. If the SPP $k$-vectors orbit in a clockwise fashion, their spin converges to the center, as shown in Figure 6.11. In addition, if the topological charge of the SPP vortex is $L=1$, then the in-plane components of the SPP fields do not vanish at the vortex core; only the out-ofplane component vanishes at the phase singularity, as predicted from Eq. 6.1-6.3. The in-plane fields instead have non-vanishing amplitudes, which orbit in the same direction as the geometric charge of the spiral; this in-plane circulation of the SPP field produces SAM in the $z$-direction.

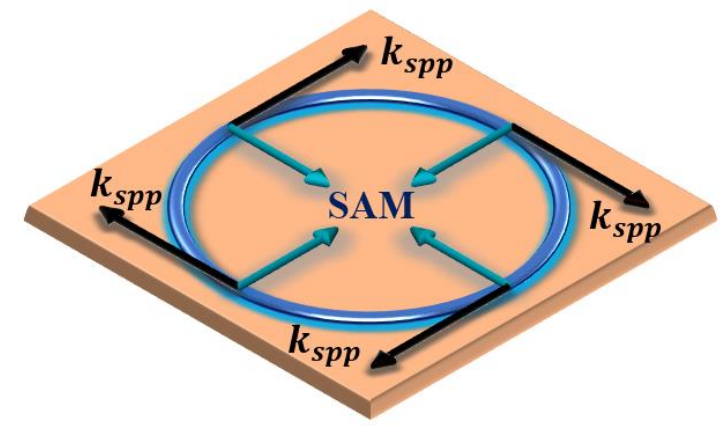

Figure 6. $11 \mathrm{~K}$-vector and spin direction of an $L=1$ SPPs vortex.

The total spin texture existing within an $L=1$ SPPs vortex core is shown in Figure 6.12. One can see that at the vortex center, SAM points up (z-direction). Away from the vortex core, the out-of-plane SAM decreases, and the SAM direction becomes mostly in-plane, pointing 
towards the center. Farther away, the SAM directions become perpendicular again, but now point down, i.e., anti-parallel with respect to the spin at the vortex core. Such continuous flipping of the spin vector resembles the texture of a magnetic Skyrmion, that forms a stable texture in ferroelectric materials with strong spin-orbit coupling. I will now refer to the optical spin texture in the SPP vortex as the optical spin Skyrmion, in analogy to the magnetic one.

A Skyrmion, is a quasiparticle proposed to model nucleon structure,[100] and its topological texture has been found in Bose-Einstein condensates, liquid crystals, and superconducting vortices.[101-103] In magnetic materials, it is established by the DzyaloshinskiiMoriya interaction. Skyrmions composed of local spins have been demonstrated to exist when a 3D spin texture is projected onto a $2 \mathrm{D}$ surface, where spins continuously rotate by $\pi$ from the center to the boundary.[104-106] A Néel type Skyrmion, in particular, has a spin rotation in the plane perpendicular to the surface, producing an integer topological charge, or Skyrmion number $N$, which is protected against perturbation by surface impurities. $[99,107]$ In addition, the motion of a topologically stable magnetic Skyrmion can be controlled by low electric currents, thus making the potential candidates for magnetic information storage and for spintronic devices.[108, 109] 


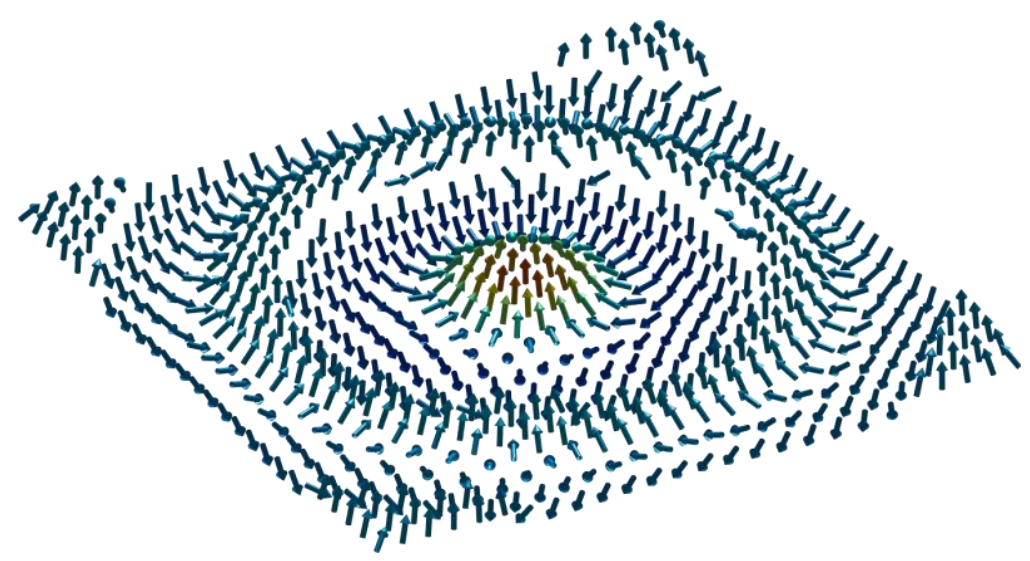

Figure 6. 12 3D spin texture of an $L=1$ SPPs vortex.

A key property of a Skyrmion is its topological charge. We can determine the topological charge of the plasmonic spin Skyrimon as follows. First, we generate a map of the Skyrmion density near the vortex core, which is defined as, [99]

$$
D=S \cdot\left(\frac{\partial S}{\partial x} \times \frac{\partial S}{\partial y}\right)
$$

where $S$ is the SAM of the SPP fields. By doing the calculation for the spin texture shown in Figure 6.12, I produce a map out the Skyrmion density as shown in Figure. 6.13, where the strong density appears as a ring surrounding the SPP vortex core. The boundary of the Skyrmion can be defined by where the out-of-plane SAM becomes purely negative, which occurs at the zero point of the $J_{1}\left(k_{s p p} R\right)$, as shown by the dashed circle in Figure 6.13. By integrating the Skyrmion within that boundary, I obtain the Skyrmion topological charge, which, numerically converges to 1. 


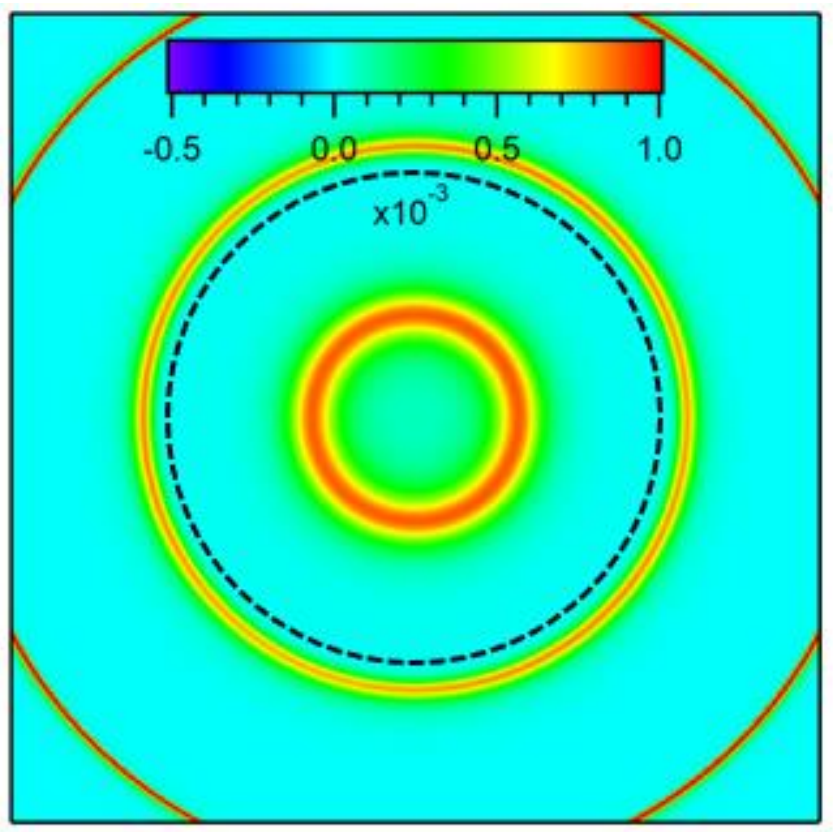

Figure 6. 13 Skyrmion density map of the $L=1$ vortex.

For an SPP vortex orbiting in a counter-clockwise manner $(L=-1)$, all the spin directions flip with respect to $L=1$, which corresponds to an anti-Skyrmion spin texture. In this case, the Skyrmion density map is inverted and its topological charge is -1 .

In Figure 6.14, I plot the calculated dynamic behavior of the topological charge of an optical spin Skyrmion in a vortex of geometric charge $L=-1$, for excitation a pulse of $25 \mathrm{fs}$, from FDTD simulations. Along with the topological charge, the in-plane SPP field at the vortex core is also plotted as a reference for when the topological charge is formed. One can see that the SPP vortex field reaches a maximum at approximately $\sim 80 \mathrm{fs}$ and has a spread of $\sim 25$ corresponding to the pulse duration. Time zero is defined by when the center of the optical pulse interacts with the AS structure, and the delay of $80 \mathrm{fs}$ is the SPP propagation time to the vortex core. The spin Skyrmion topological charge reaches to -1 at $\sim 50 \mathrm{fs,} \mathrm{which} \mathrm{indicates} \mathrm{that} \mathrm{a} \mathrm{stable} \mathrm{spin} \mathrm{texture} \mathrm{is}$ 
formed even before the vortex field amplitude has reached its maximum. As the SPP fields orbit around the core, the topological charge persists for over $\sim 100$ fs. This is because SPPs exist on femtosecond time scale and therefore, the SAM pseudovectors are defined longer than the laser pulse. The topological charge is really a property of the SPP generation geometry, and when it is established and decays, is determined in the calculation by the time scale when the SPP field exists above the noise level of the calculation.

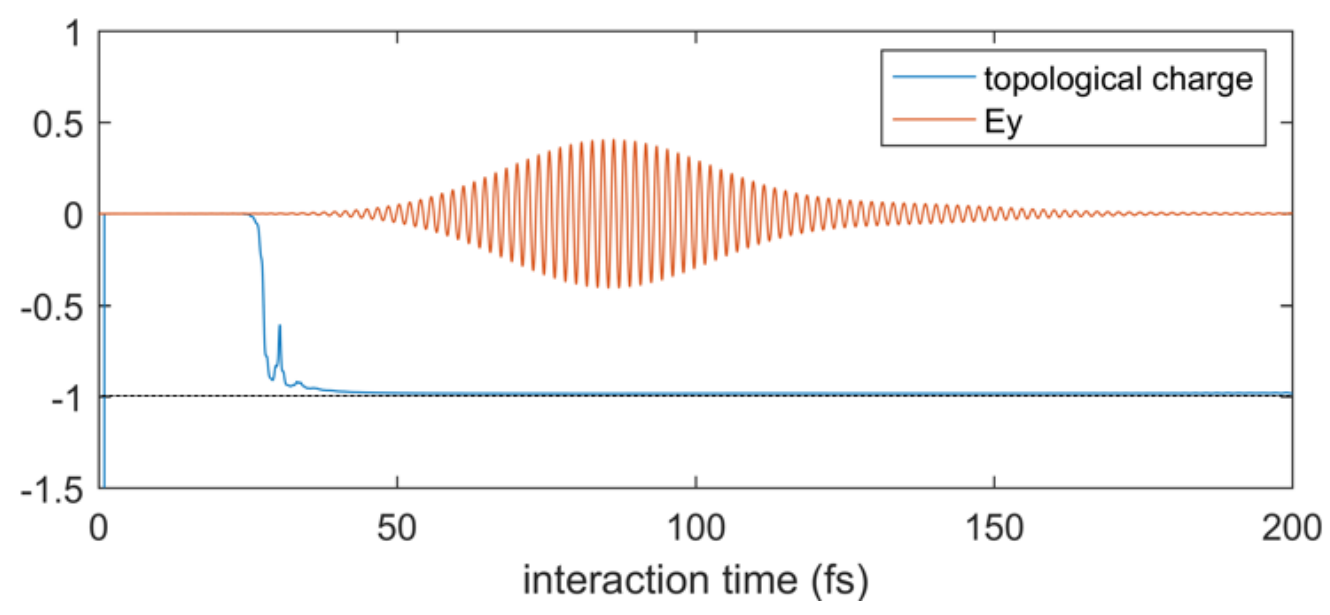

Figure 6. 14 Topological charge dynamics of optical spin Skyrmion.

\subsubsection{Optical Spin Skyrmion at an SPPs Vortex Core}

Next, I will discuss the topological character of the SAM associated with an SPP vortex formed by illuminating an AS structure having a geometric charge $m=2$ with linearly polarized light. One should also expect a plasmonic SAM Skyrmion to form at the vortex core because of 
the orbiting SPP fields, as in the above calculation. By performing FDTD simulations for an $m=2$ AS structure with $550 \mathrm{~nm}$ linearly polarized light, I calculate the Skyrmion density of the SAM that forms within the SPP vortex core, which is shown in Figure 6.15. Compared with the case of circularly polarized light excitation of an $L=1$ structure in section 6.3.1, the Skyrmion density map now does not have a rotational symmetry, but only a mirror symmetry (left/right) with respect to the core. This symmetry can be mostly attributed to the linearly polarized excitation, which has electric field polarizations in the symmetry direction. Defining the boundary as indicated by the hour glass shaped boundary [L-line in Figure 6.16(b)], we can determine a topological charge of $+1 / 2$ for the SAM texture.

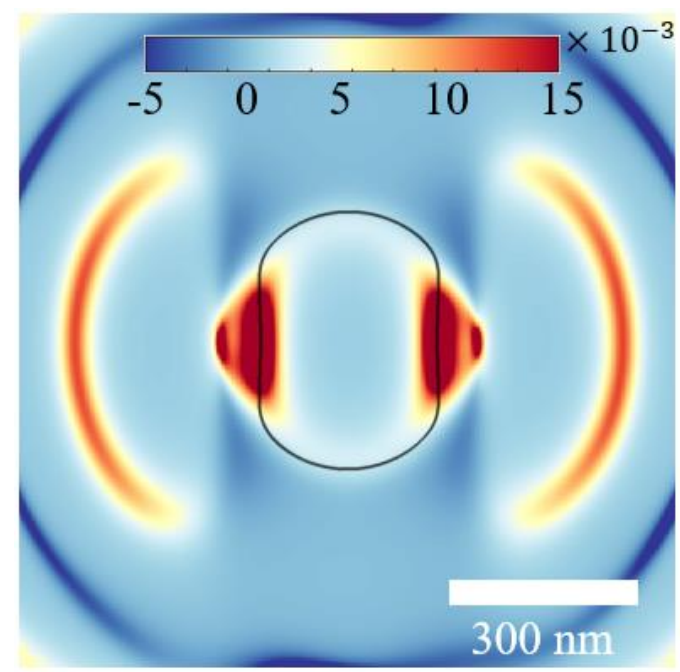

Figure 6. 15 Skyrmion density of the optical SAM asscociated with the SPPs vortex launched by $550 \mathrm{~nm}$, linearly (horizontally) polarized light on Ag surface. 


\subsubsection{Locating Optical Spin Skyrmion}

Although it is hard to directly measure the SAM of a light field directly at a metal surface to image the Skyrmion, one can use additional properties to locate the optical SAM Skyrmion. For this purpose, I introduce two types of optical singularities here.[83, 110] The first type is an L-line singularity, which defines the location where the in-plane SPP fields have linear polarizations. The second type is the C-line singularity, which defines the region where the fields have purely circular polarization. To locate Skyrmions, I define a map of L-line singularities for the in-plane component of the SPP vortex field. These singularities are important because there is no in-plane field circulation, thus the SAM is purely in-plane. The L-line map where the z-component of SAM is zero coincides with the strongest Skyrmion density, because it is dominated by the spatial derivative of the SAM.

To obtain the L-line map, I compute the ellipticity of the in-plane electric fields in the near vortex region. For $L=1$ SPP vortex excited by RCP light (the case in 6.3.1), the L-line map is shown in Figure 6.16 (a), while for the $m=2$ vortex excited by linearly polarized light (the case in 6.3.2), the L-line map is shown in Figure 6.16 (b). Evidently, for circularly polarized light excitation, the L-line map consists of concentric rings, with the inner most L-line, as expected, being located around the major ring of the SPPs vortex. The second L-line nearly coincides with the second zero point of the Bessel function, $J_{1}\left(k_{s p p} R\right)=0$, thus it marks the Skyrmion boundary.

In the case of linear excitation, the L-line distribution is more complicated, but one can mark the primary ring of the L-line, which can define the optical SAM Skyrmion that has a unity topological charge. 

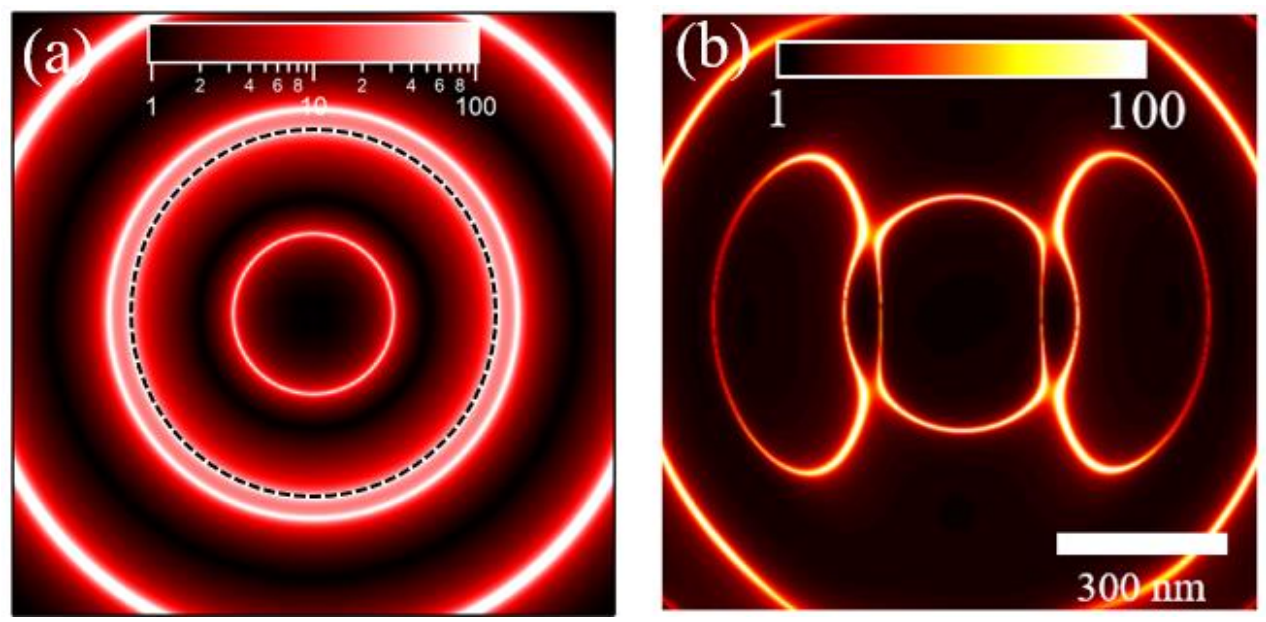

Figure 6. 16 L-line map of the $L=1$ SPP vortex excited by RCP light. (b) The L-line map of the $m=2$ SPP vortex cited by the lineary polarized light.

Next, I will demonstrate on how to locate the SAM Skyrmion experimentally in the case of linearly polarized light excitation of the $m=2$ AS coupling structure (section 6.3.2). To experimentally observe the L-line distribution, we extract the plasmonic flow of the SPP vortex field that is measured by ITR-mP-PEEM. Unlike the PEEM images in Figure 6.10, I further process the images by Fourier transforming the time-dependent PEEM data into the frequency domain, which produces a set of frequency spectra for each spatial image pixel. Figure 6.17 plots such frequency domain spectrum. Then, I collect only the first-order oscillatory component, by keeping the Fourier amplitude in the dashed box; with this amplitude, I perform an inverse Fourier transform, which gives the filtered real space PEEM data on the plasmonic vortex. In this way, I extract the time-dependent fields only. The resulting PEEM images, corresponding to Fourier filtering of the data in Figure 6.10, are shown in 6.18. 


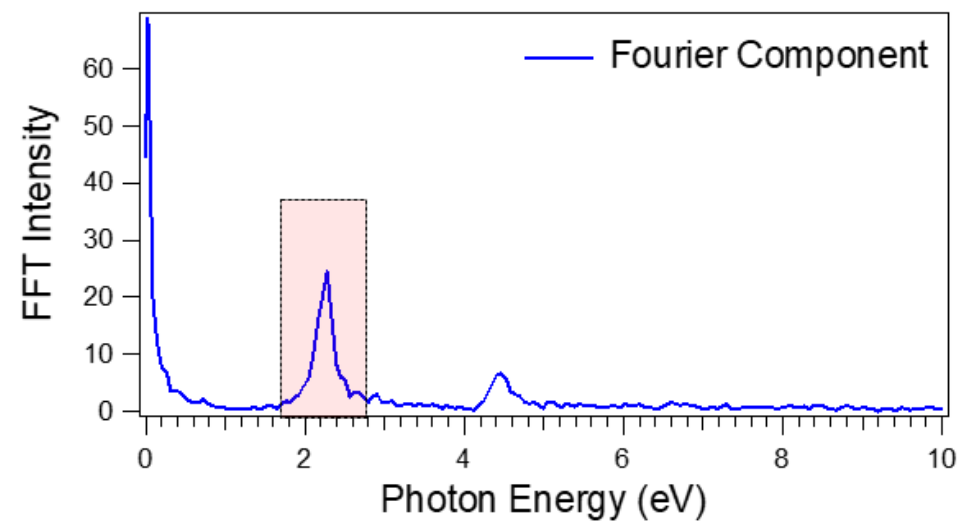

Figure 6. 17 Fourier spectrum of the time-dependent PEEM data. The dashed box maks the first-order oscillitory photoemission component.
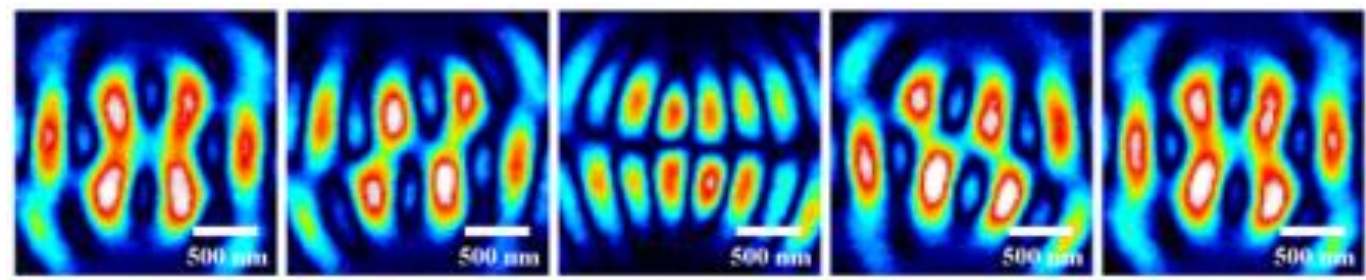

Figure 6. 18 Fouier filtered PEEM images, with time-delay corresponding to the PEEM images in Figure 6.10.

Next, I apply the Horn-Schunck (HS) algorithm to the filtered PEEM images.[111] The HS algorithm calculates the spatio-temporal derivatives of the PEEM image sequence and gives a vector of the intensity flow, which mimics the in-plane electric field polarizations of the SPP fields. Finally, the polarization ellipticity is calculated based on the vectors of the intensity flow, which 
gives the experimental L-line map. An L-line map for the $m=2$ SPPs vortex excited by linearly polarized light is shown in Figure 6.19. One can see that it has excellent agreement with the calculated L-line map in Figure 6.16(b). Therefore, such method can be used to experimentally locate the optical SAM Skyrmion discovered and introduced in this Chapter.

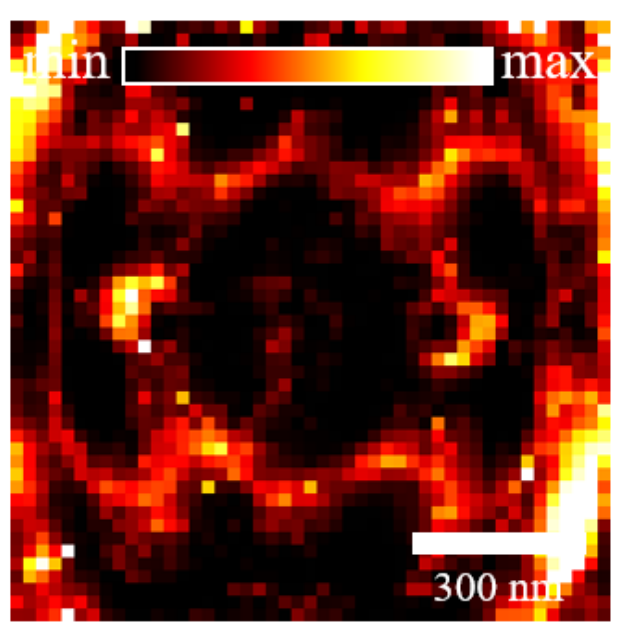

Figure 6. 19 An optical flow image determining the experimental observed L-line map of the $m=2$ SPP vortex excited by linearly polarized light. 


\subsection{Summary and Perspectives}

To summarize, I have employed interferometric time-resolved multi-photon photoemission microscopy, combined with wavelength tunable femtosecond lasers, to study the amplitudes, phases, spins and dynamics of surface plasmon polariton fields at various nano/microstructures ranging from epitaxially grown silver wires/islands to structured Archimedean spirals. I conclude that light of various polarizations can couple into SPP mode in two ways; when the electric field is oscillating in the surface normal direction, it couples into the transverse SPP mode, whereas when it is oscillating in the surface plane and perpendicular to the coupling edge, it couples into the longitudinal SPP mode. The coupling of two modes together can explain the general SPP excitation mechanisms, such as the SAM dependent SPPs coupling, where the plasmonic quantum spin Hall effect arises from the superposition of transversely and longitudinally coupled SPPs. In addition, the SPPs dynamics associated with differently polarized excitation light remains time-reversal symmetric in a medium, such as Ag. Next, I described the normal-incident light PEEM measurements, where I can illuminate sample surfaces with a defined phase front, to study the plasmonic vortex generation. By imaging the dynamics of the SPP waves launched from Archimedean spiral structures with both the circularly and linearly polarized light, I found that the SAM of light is not necessary to impart OAM to SPP wave fronts. The Archimedean spiral structures can be constructed with geometric charge so that the formed SPP waves still form a vortex without supplying the external SAM of light. Finally, based on numerical simulations, I found that associated with the plasmonic vortices there is a novel Bosonic quasiparticle, an optical spin Skyrmion, which is topologically stable over pulse excitation. This 
discovery opens a new field of ultrafast topological plasmonics that can be investigated by future generations of students. 


\section{Appendix A Time-Resolved PEEM Software}

The ITR-mP-PEEM experiment is performed by scanning a pump-probe pulse pair via a Mach-Zehnder Interferometer, with the inter-pulse delay tuned by a nanometer precision piezoelectric. The voltage applied to the piezoelectric is controlled through a LabVIEW program, or virtual instrument (VI).

Figure A.1 shows the front panel of the voltage control VI to the piezoelectric. The VI generates a triangle wave of voltage, of range $0 \sim 10 \mathrm{~V}$, with preset offset, amplitude and asymmetry. The asymmetry is designed to be the time of the upward trend of the triangle with respect to the total period. The frequency determines the frequency of the oscillation of the piezoelectric, which typically runs on the order of $10 \mathrm{~Hz}$ for optical alignment. Its block diagram is shown in Figure A.2.

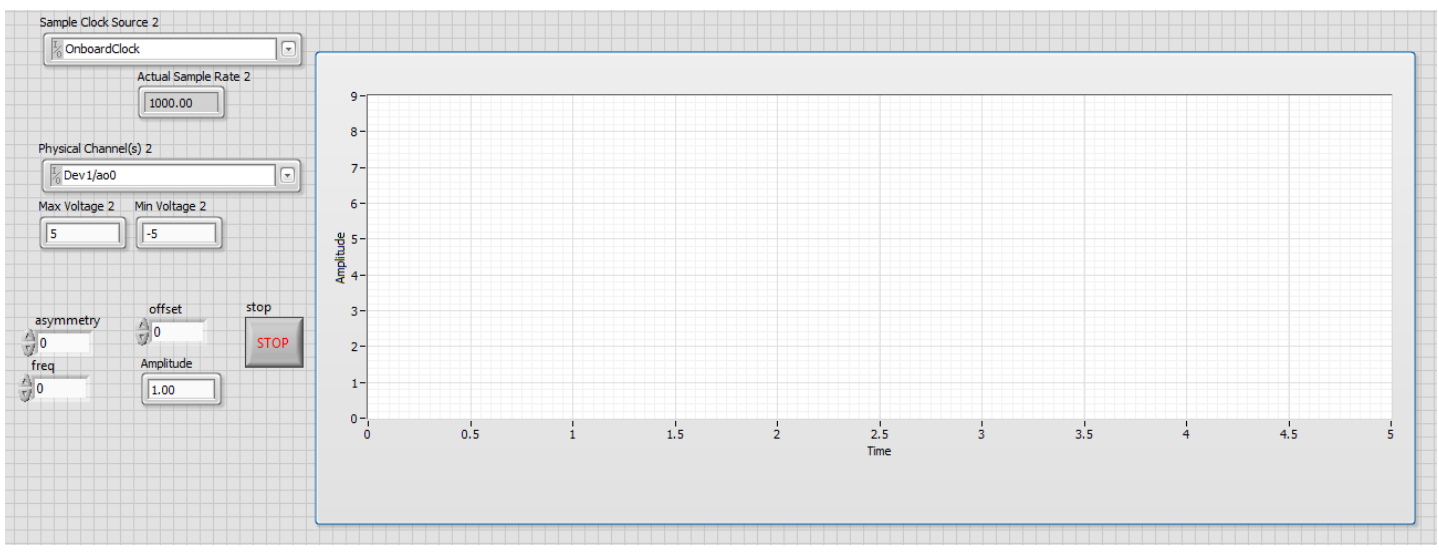

Figure A. 1 Front panel of Labview VI for Piezoelectric voltage generation. 


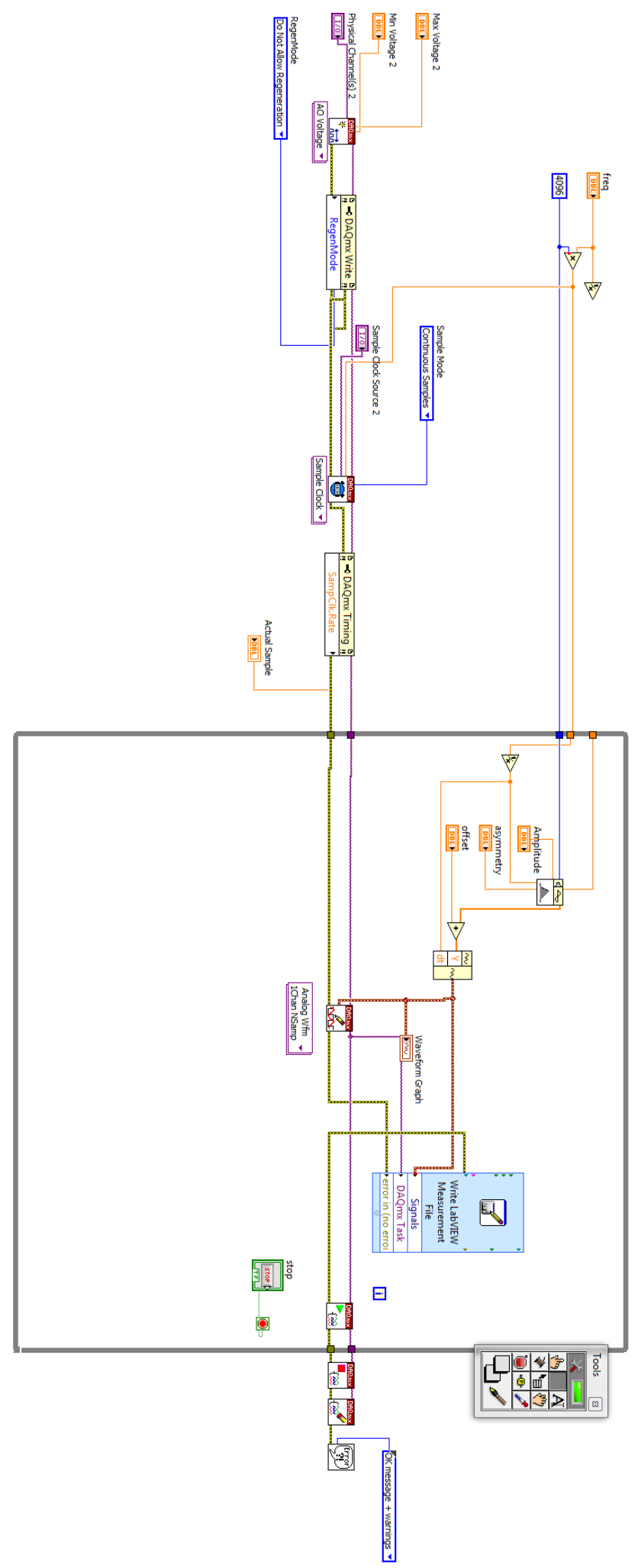

Figure A. 2 Block diagram of the piezoelectric voltage generation VI in Figure A.1. 
The ITR-mP-PEEM image acquisition software is also implemented using LabVIEW VIs, through directly access the detector with SensiCam software control. Figure A.3 shows the front panel of the acquisition software. The left side lists multiple parameters needed for the PEEM frames, while the right side is the image screen. The region of interest (ROI) parameters determine how large area of the detector is going to be imaged. The maximum range is $(1,43) \times(1,33)$. The exposure time determines how long an image is acquired, typical acquisition time is $500 \sim$ $800 \mathrm{~ms}$. While scanning with pump-probe delay, the VI incorporate with the voltage control VI to tune the piezo electric. At each time step (frame of PEEM), the number of images needed at that step is determined by the \# of images parameter. The total number of images is determined by the \# of samples, which together with the maximum voltage (amplitude parameter) also determines the voltage increment used in PEEM experiment.

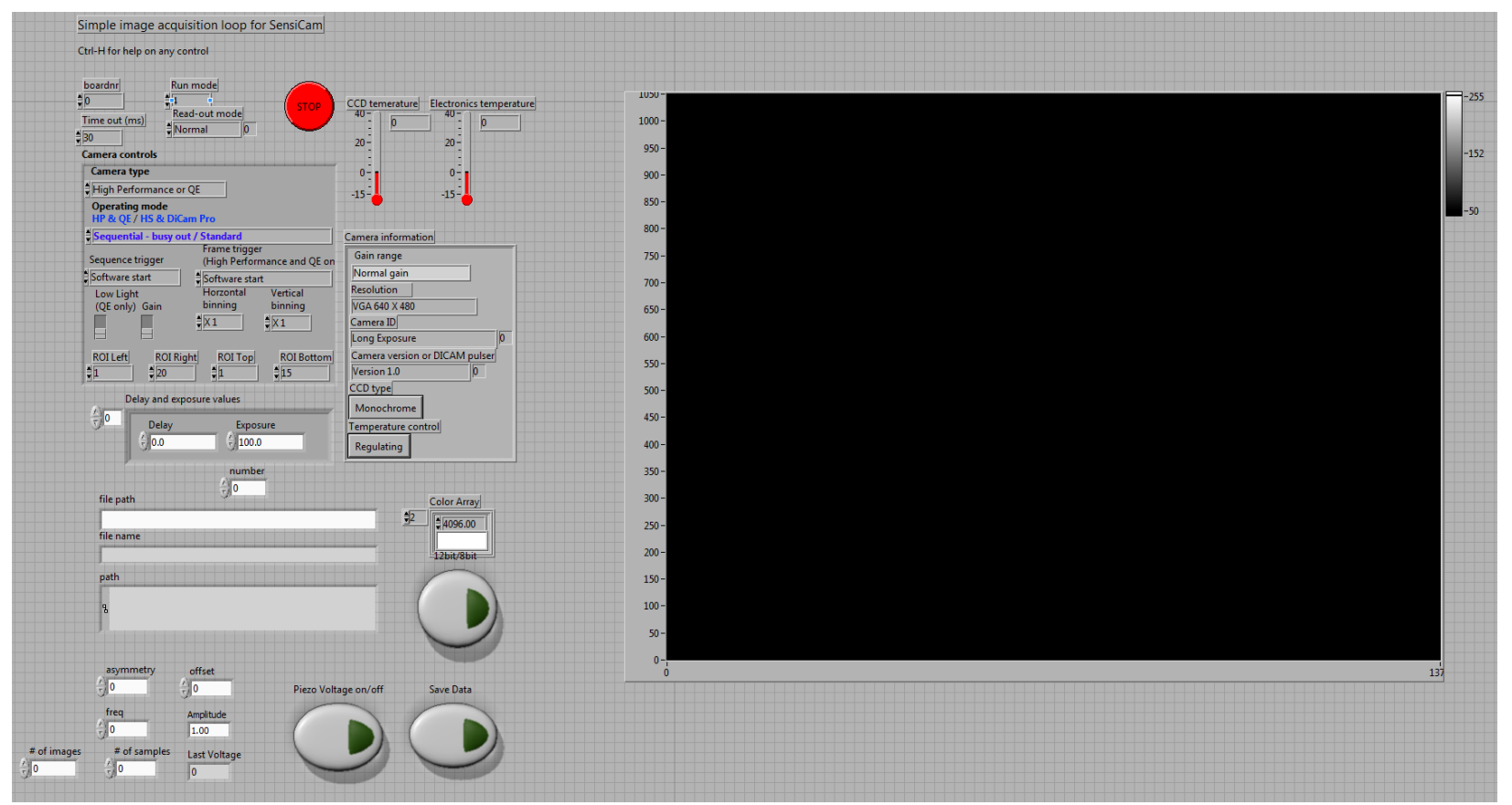

Figure A. 3 Front panel of the TR-PEEM image acquisition software. 
Figure A.4 shows the first layer of the block diagram of the acquisition VI. This VI initiate the operation of the SensiCam CCD camera we use in PEEM, by feeding the desired parameters, such as region of interest. If the CCD camera is successfully turned on, then an indicator signal is transmitted to the second layer of the VI, which will trigger the image acquisition.

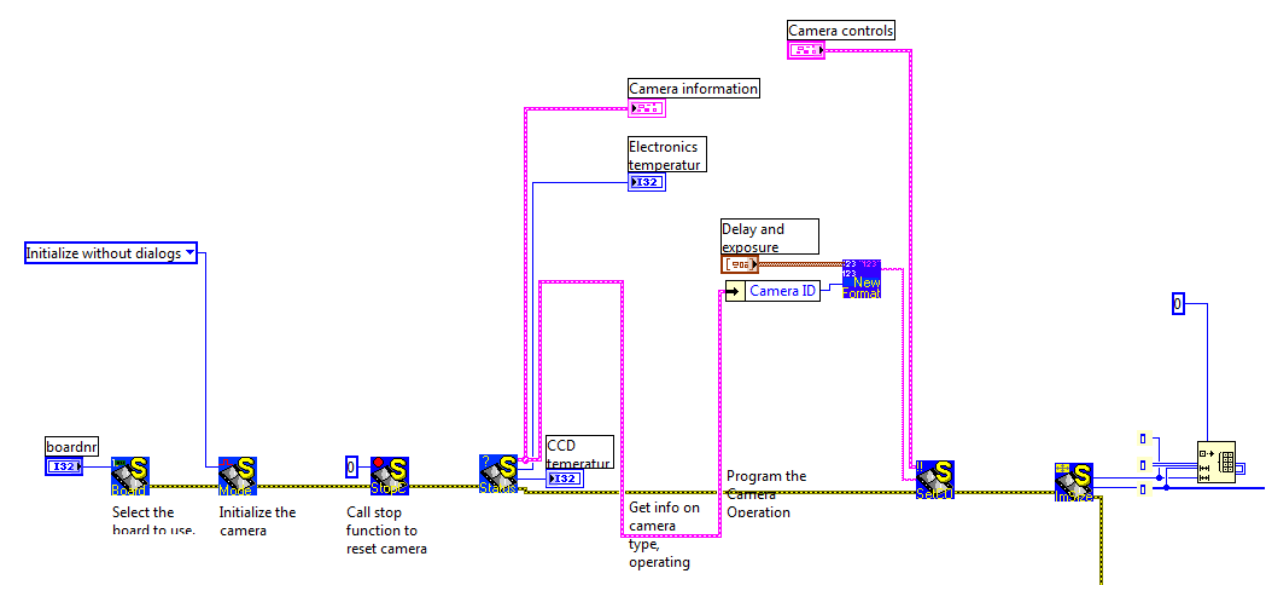

Figure A. 4 Block diagram of the TR-PEEM image acquisition software, part I.

Figure A. 5 shows the block diagram of the second acquisition layer. In this layer, the preset parameters for the control of the piezoelectric is applied, and generate a step-wise triangle voltage wave form. The image acquisition is synchronized with the piezoelectric generation. After the voltage is applied, the VI starts to acquire PEEM image, which is then stored in a TIFF image as a $2 \mathrm{D}$ integer matrix with 32 bit. At each time step, the voltage is held constant, and the VI outputs a series of images based on the input parameters. 


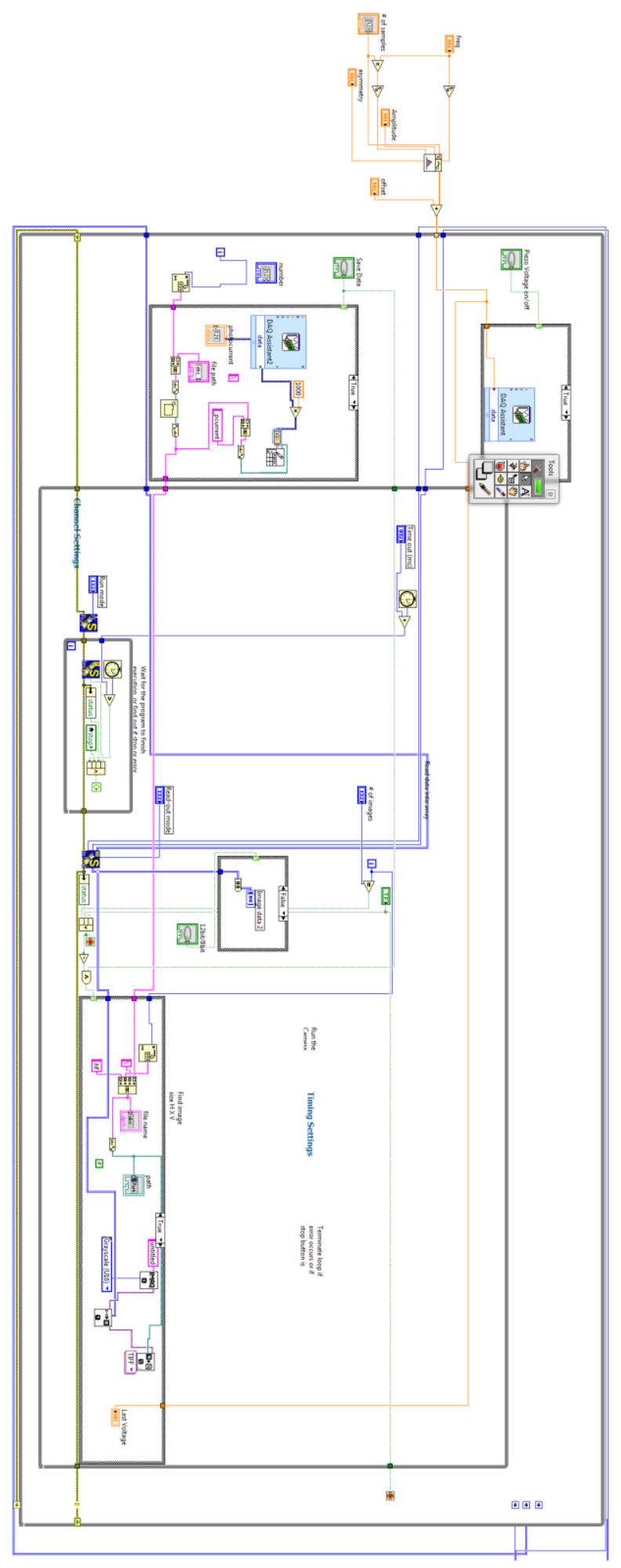

Figure A. 5 Block diagram of the TR-PEEM image acquisition software, part II. 


\title{
Appendix B Huygens Principle Simulation Package
}

The following code is an implementation of the Huygens principle calculations introduced in Chapter 3. It employs $\mathrm{p}$ - and s- polarizations into consideration, to mimic the vectorial excitation of the SPP waves.

\author{
\#include "mpi.h" \\ \#include <stdio.h> \\ \#include <math.h> \\ \#include <string.h> \\ \#include <stdlib.h> \\ \#include "proto.h"
}

/* Light field, plasmon field and their interference*/

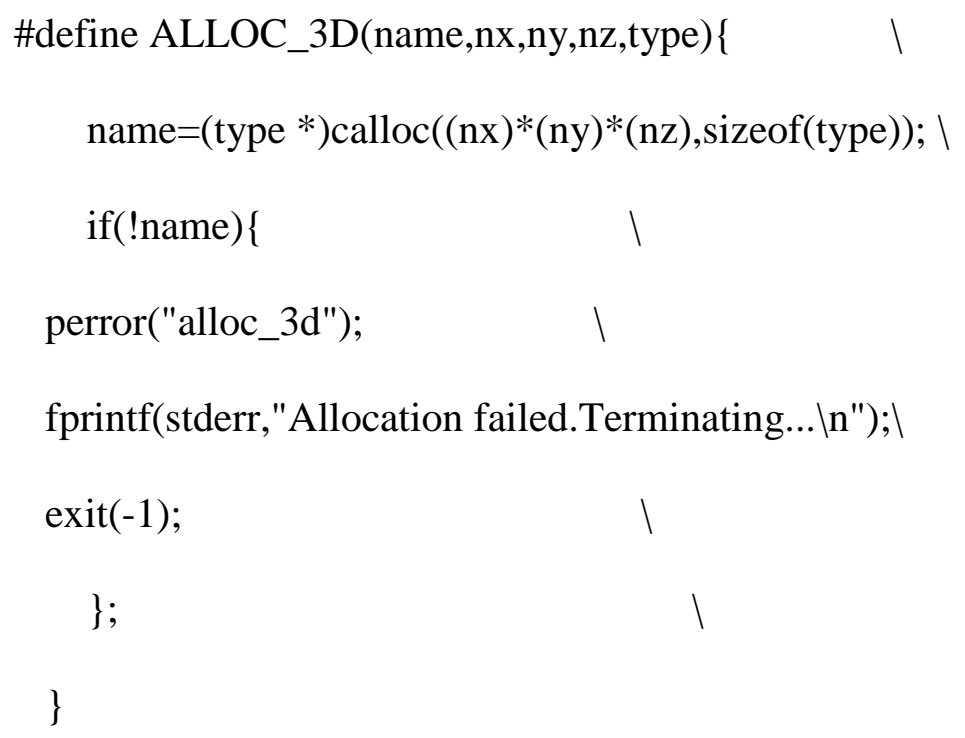




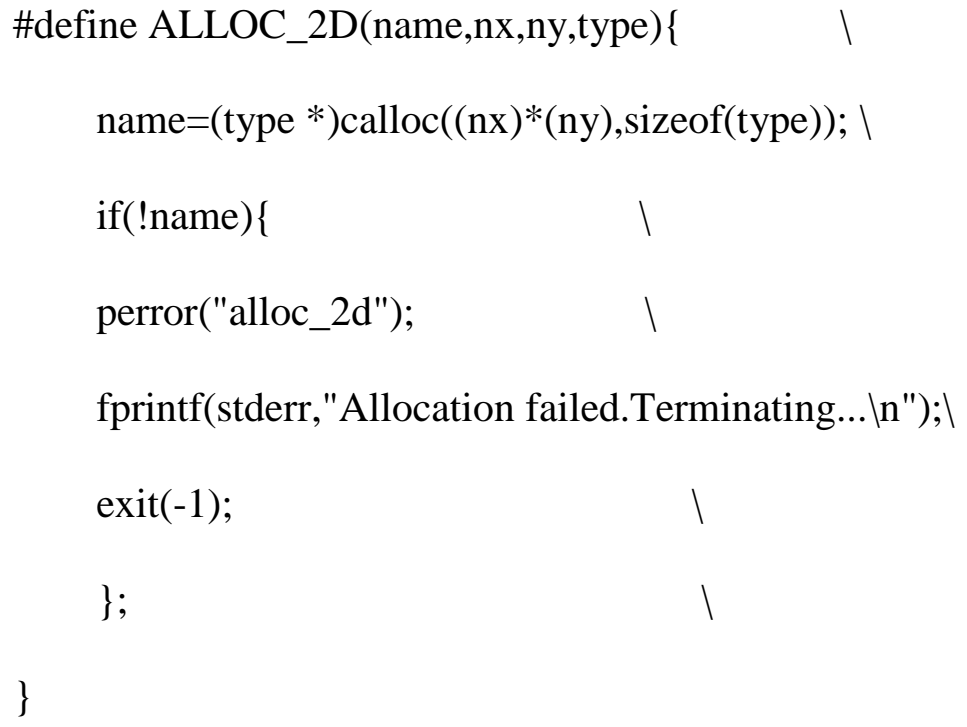


\#define eps_infi 6.8635

\#define Reff $20 / *$ excitation efficiency----one of fitting parameter*/ \#define Thi (0.0*TWO_PI) /*phase delay between spp and light -----fitting parameter*/

\#define C $299792458.0 \quad$ /*light speed*/

\#define N $512 \quad$ /*time steps*/

\#define Tmin (-250e-15) /*start point (fs) in time-domain*/

//\#define Dt $20.0 \quad$ /*pump-probe delay*/

\#define Tmax (250e-15) /*end point (fs) in time-domain*/

/*spacial steps and length it represents*/

\#define Num_X 400

\#define Num_Y 180

\#define DeltaX (5e-9)

$/ *$ define lens structure*/

\#define $\operatorname{Rad} 183$ /*in unit of DeltaX*/ 
\#define Ns 360 /*number of source pointss*/

\#define fx $230 / * \mathrm{x}$ coordinate of the focus point*/

\#define fy $200 / *$ y coordinate of the focus point*/

int main(int $\operatorname{argc}, \quad$ char *argv[])

\{

/*** set MPI ***/

int numtasks, taskid, dest, source;

MPI_Status status;

MPI_Init(\&argc, \&argv);

MPI_Comm_size(MPI_COMM_WORLD, \&numtasks);

MPI_Comm_rank(MPI_COMM_WORLD,\&taskid);

printf ("MPI task \%d has started...In", taskid);

int Core_X $=4$, Core_Y $=9$;

int Task_number $=$ Core_X*Core_Y;

int Nx = Num_X/Core_X, Ny = Num_Y/Core_Y;

int L2[36]; 
double Ratio;

double Dt;

int frame;

void wrtraw(double *fld,int size_x,int size_y,char *filename);

double *P_light, ${ }^{*} \mathrm{P} \_$spp, ${ }^{*} \mathrm{P} \_$total, ${ }^{*}$ Intensity;

double Omega,Omega_j=0.0,Sigma,Gamma,K,Kx $[N]=\{0.0\}$,

$\operatorname{Kspp} \_\mathrm{R}[\mathrm{N}]=\{0.0\}, \mathrm{Kspp} \_\mathrm{I}[\mathrm{N}]=\{0.0\}$

double Ktemp_R,Ktemp_I,R_temp,Phi_temp;

double epsAg_real $=0.0$, epsAg_img $=0.0$;

double timestep,fs; $\quad I^{*}$ time interval beteween sampling points and the

corresponding sampling rate*/

double

f[N],R[N],Phi[N],R_prime[N],Phi_prime[N],R_spp[N],Phi_spp[N],R_spp_prime[N],Phi_spp_pr

ime $[\mathrm{N}] ; \quad / *$ amplitude and phase for the frequency spectrum*/

// printf("please input the pump-probe delay (in unit of TWO_PI):");

// $\quad$ scanf("\%lf", \&Dt); 
double L,displaceX,incremental_left,incremental_right;

double Theta,Dtheta;

double Delay;

int $\mathrm{i}, \mathrm{j}, \mathrm{m}, \mathrm{n}, \mathrm{k}$;

int choice $=0$;

int $\mathrm{f}$ center $=0$;

double $\operatorname{Rmax}=0.0$;

double t,t_prime, $\mathrm{x}$;

double $(* \mathrm{E})[2],\left(* \mathrm{E} \_\mathrm{spp}\right)[2] ; \quad \quad / * \mathrm{n} * 2$ (real and imaginary part) array of time-

domain signal $* /$

double $(* \mathrm{~F})[2],\left({ }^{*} \mathrm{~F}_{-} \mathrm{Spp}\right)[2] ; \quad \quad \quad / * \mathrm{n} * 2$ (real and imaginary part) array of frequency-

domain signal $* /$

char file1[100],file2[100],file3[100],filename1[100],filename2[100];

FILE $* \mathrm{fp}, * \mathrm{fp} 2, * \mathrm{fp} 3$

$/ * \mathrm{E} / \mathrm{F} / \mathrm{E} \_$spp/F_spp $[\mathrm{i}][0] /[\mathrm{i}][1]$ stores, respectively, the real and imaginary part the of field and frequency*/

$$
\mathrm{E}=(\text { double }(*)[2]) \operatorname{malloc}(2 * \mathrm{~N} * \operatorname{sizeof}(\text { double }))
$$


$\mathrm{F}=($ double $(*)[2]) \operatorname{malloc}(2 * \mathrm{~N} * \operatorname{sizeof}($ double $))$;

E_spp $=($ double $(*)[2]) \operatorname{malloc}(2 * \mathrm{~N} * \operatorname{sizeof}($ double $))$;

F_spp $=($ double $(*)[2]) \operatorname{malloc}(2 * \mathrm{~N} * \operatorname{sizeof}($ double $))$;

ALLOC_3D(P_light,Nx,Ny,N,double);

ALLOC_3D(P_spp,Nx,Ny,N,double);

ALLOC_3D(P_total,Nx,Ny,N,double);

ALLOC_2D(Intensity,Nx,Ny,double);

for $($ frame $=0$; frame $<1$; frame ++$)\{$

for $(\mathrm{m}=0 ; \mathrm{m}<\mathrm{Nx} ; \mathrm{m}++)$

for $(\mathrm{n}=0 ; \mathrm{n}<\mathrm{Ny} ; \mathrm{n}++)$

for $(j=0 ; j<N ; j++)\{$

$\operatorname{Pspp}(m, n, j)=0.0 ;$

\}

// $\mathrm{Dt}=$ frame/13.3;

$\mathrm{Dt}=0$;

Delay $=\mathrm{D} t^{*}$ centerwavelength/C;

printf(" Dt delay= $\% 1.20 \mathrm{f} \% 1.20 \mathrm{f} \backslash n ", D t$, Delay); 
timestep $=(\operatorname{Tmax}-\mathrm{Tmin}) /(\mathrm{N}-1)$;

fs=1.0/timestep;

Omega=TWO_PI*C/centerwavelength;

Sigma $=F W H M /(2.0 * \operatorname{sqrt}(\log (2.0)))$;

/*calculate $\mathrm{x}$ and $\mathrm{y}$ coordinate of the sources points*/

double $(* \mathrm{~S})[2]$;

$\mathrm{S}=($ double $(*)[2])$ malloc $(2 * \mathrm{Ns} *$ sizeof(double $)) ; / *$ store $\mathrm{x}$ and $\mathrm{y}$ coordinates for the source points*/

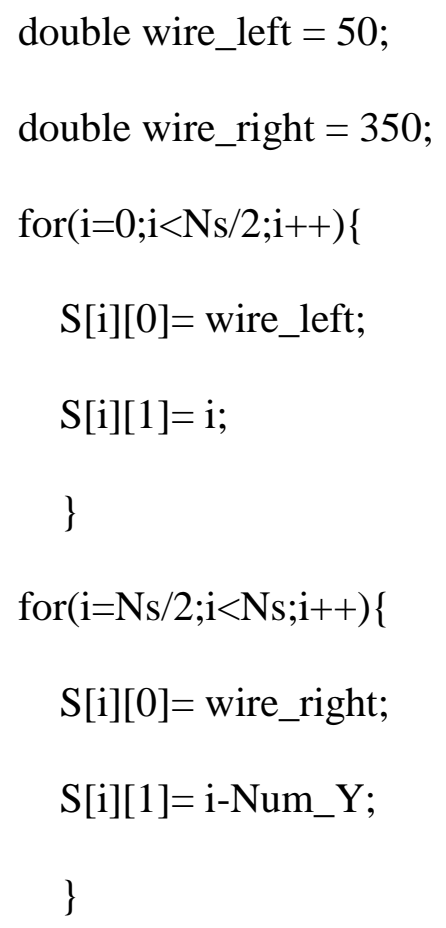


/*construct the light pulse*/

/* printf("Output file for original light pulse E(n):");

scanf("\%s", file);

*/

$$
\mathrm{fp}=\text { fopen("Elight", "w"); }
$$

for $(\mathrm{i}=0 ; \mathrm{i}<\mathrm{N} ; \mathrm{i}++)$

\{

$$
\mathrm{t}=\mathrm{i} * \text { timestep+Tmin; }
$$$$
\mathrm{E}[\mathrm{i}][0]=\sin \left(\mathrm{Omega}^{*} \mathrm{t}\right) * \exp (-(\mathrm{t} / \text { Sigma }) *(\mathrm{t} / \text { Sigma }))+\quad+\quad \sin \left(\mathrm{Omega}^{*}(\mathrm{t}-\right.
$$

Delay $))^{*} \exp (-((\mathrm{t}-$ Delay $) /$ Sigma $) *((\mathrm{t}-$ Delay $) /$ Sigma $)) ;$

$$
\mathrm{E}[\mathrm{i}][1]=0.0
$$

fprintf(fp, "\%23.15e \%23.15e \%23.15eln", t, E[i][0], E[i][1]);

\section{\}}

fclose(fp);

/*construct the excited SPP wavepacket--Drude Model*/

for $(\mathrm{i}=0 ; \mathrm{i}<\mathrm{N} ; \mathrm{i}++)$

\{

$$
\begin{aligned}
& \text { E_spp }[i][0]=0.0 ; \\
& \text { E_spp }[i][1]=0.0 ;
\end{aligned}
$$

\} 


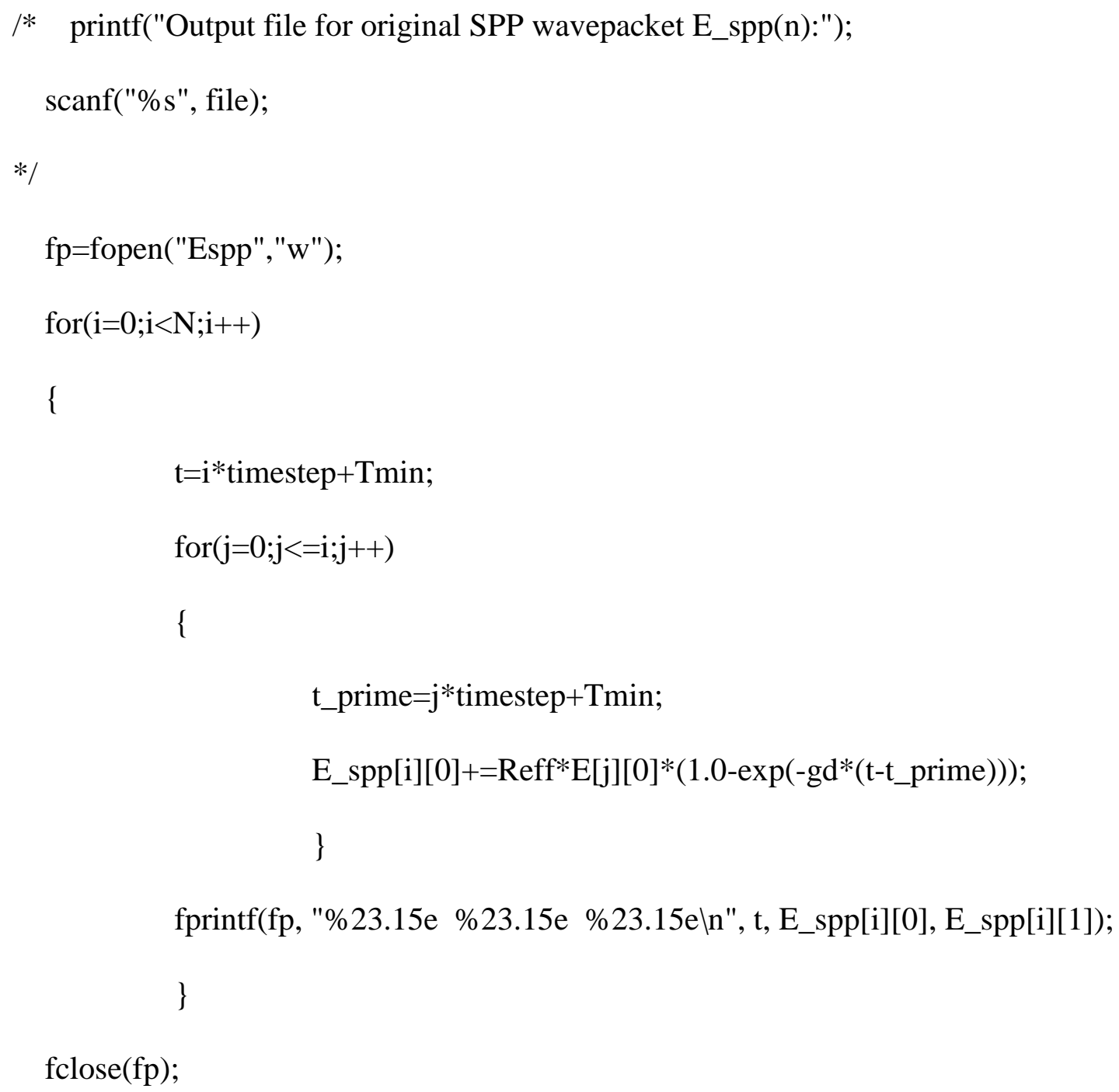




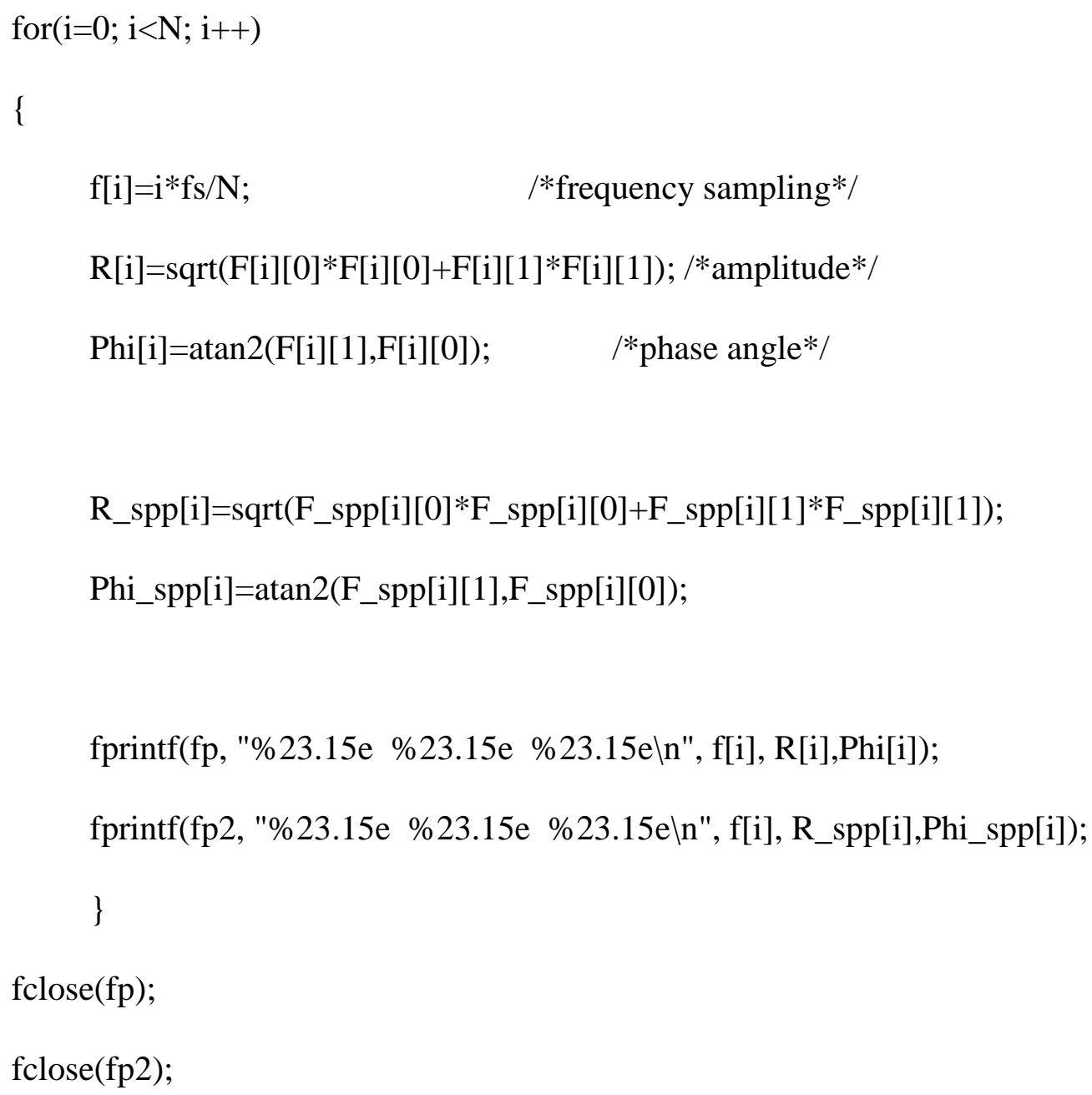

printf("the peak center of frequency spectrum is at:\%d\n",f_center); printf("the peak frequecy values is:\%23.15eln", f[f_center]); 


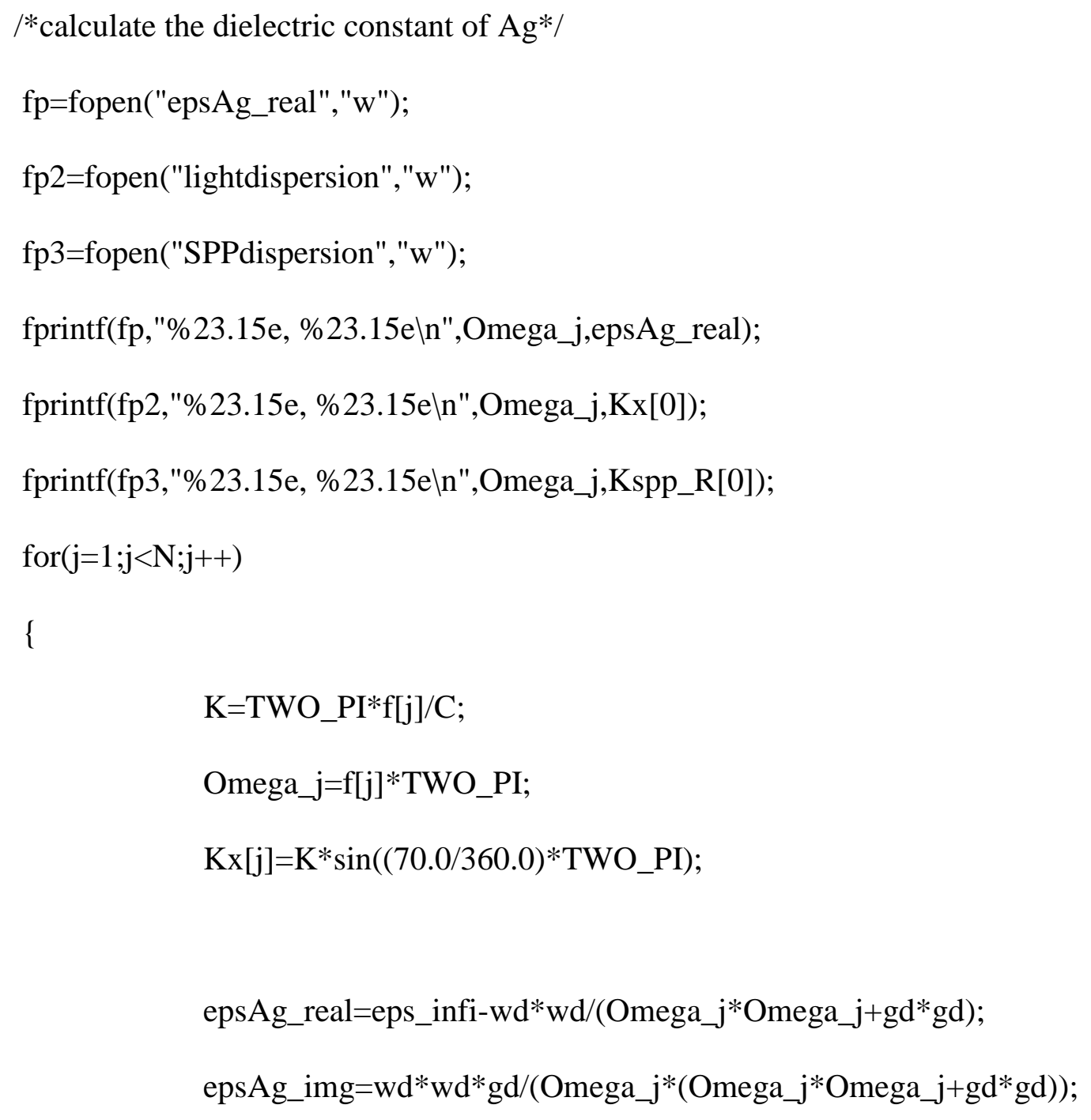

Ktemp_R=(epsAg_real*(11.7+epsAg_real)+epsAg_img*epsAg_img $) /((11.7+$ epsAg_real $) *(11.7$ +epsAg_real)+epsAg_img*epsAg_img);

Ktemp_I=epsAg_img/((11.7+epsAg_real)*(11.7+epsAg_real)+epsAg_img*epsAg_img); R_temp=sqrt $($ Ktemp_R*Ktemp_R+Ktemp_I*Ktemp_I); Phi_temp=atan2(Ktemp_I,Ktemp_R); 
if $\left((\mathrm{j}>=1) \& \&\left(\mathrm{j}<=\left(\mathrm{f} \_\right.\right.\right.$center +100$\left.\left.)\right)\right) / *$ only sample the postive frequency

around f_center where Drude model applies*/

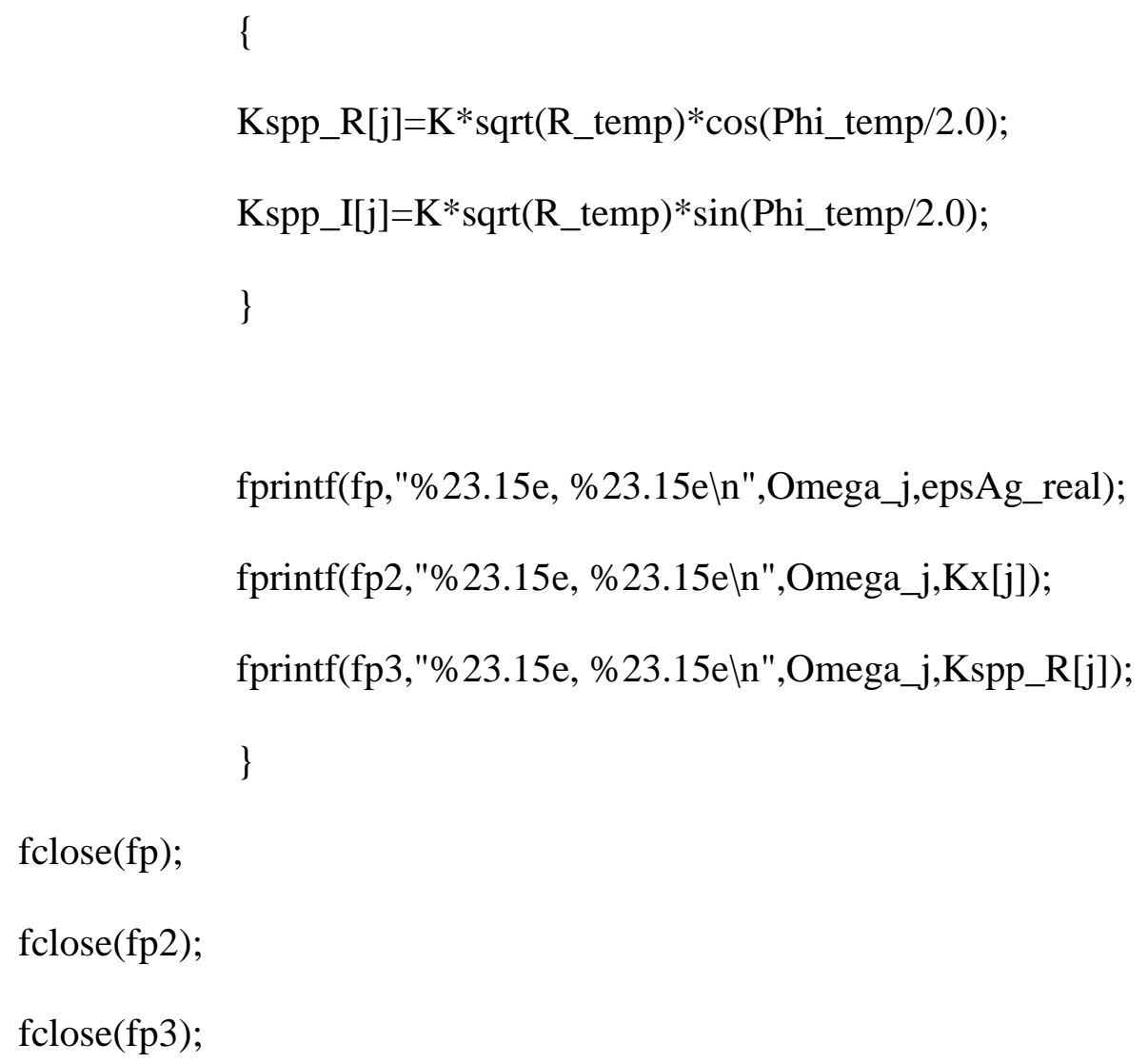


$\mathrm{x}=\left(\mathrm{m}+\right.$ taskid\%Core $\left.\_\mathrm{X} * \mathrm{Nx}\right) *$ DeltaX;

for $(\mathrm{j}=0 ; \mathrm{j}<\mathrm{N} / 2 ; \mathrm{j}++)$

Phi_prime[j]=Phi[j]-(Kx[j]** $\left.{ }^{*}\right)$;

$1 *$ set the later half part of the frequency spectrum as the complex conjugate of its front half part

---account for the negative frequency*/

for $(\mathrm{j}=(\mathrm{N} / 2+1) ; \mathrm{j}<\mathrm{N} ; \mathrm{j}++)$

Phi_prime[j]=-Phi_prime[N-j];

/*convert to cartesian coordinate*/

for $(\mathrm{j}=0 ; \mathrm{j}<\mathrm{N} ; \mathrm{j}++)\{$

$\mathrm{F}[\mathrm{j}][0]=\mathrm{R}[\mathrm{j}]{ }^{*} \cos ($ Phi_prime$[\mathrm{j}])$;

$\mathrm{F}[\mathrm{j}][1]=\mathrm{R}[\mathrm{j}] * \sin ($ Phi_prime[j]);

\}

$/ *$ do the inverse fft to recover the propagated light wave packet*/ ifft(N,E,F);

for $(\mathrm{j}=0 ; \mathrm{j}<\mathrm{N} ; \mathrm{j}++)$

$\operatorname{Plight}(m, n, j)=E[j][0]$;

\}

$\mathrm{k}=-1$; 
for $(\mathrm{i}=0 ; \mathrm{i}<\mathrm{Ns} ; \mathrm{i}++)\{$

/// calculate additional contribution from a certain phase delay within the wire if $(\mathrm{i}<\mathrm{Ns} / 2)\{$

if $(\mathrm{k} \% 2==0)\{$

displaceX $=\mathrm{m}+$ taskid\%Core_X*Nx - wire_left $+($ wire_right - wire_left $) * \mathrm{k}$;

\}

else \{

displaceX $=\mathrm{m}+$ taskid\%Core_X*Nx - wire_left + (wire_right $-(\mathrm{m}+$ $\left.\operatorname{taskid\% Core\_ X*Nx)}\right) *(k+1)+((m+$ taskid\%Core_X*Nx $)-$ wire_left $) *(k-1)$;

\}

$\mathrm{L}=\operatorname{sqrt}\left(\right.$ displaceX ${ }^{*}$ displaceX $+(\mathrm{n}+\mathrm{L} 2[\text { taskid }]-\mathrm{S}[\mathrm{i}][1])^{*}(\mathrm{n}+\mathrm{L} 2[$ taskid $]-$

$\mathrm{S}[\mathrm{i}][1])) *$ DeltaX;

$$
\begin{aligned}
& \text { for }(\mathrm{j}=0 ; \mathrm{j}<\mathrm{N} ; \mathrm{j}++)\{ \\
& \operatorname{if}\left((\mathrm{j}>=1) \& \&\left(\mathrm{j}<=\left(\mathrm{f} \_ \text {center }+100\right)\right)\right)\{\quad \quad / * \text { only works around the }
\end{aligned}
$$

spectrum frequency where the dielectric constant is a smooth function*/

Phi_spp_prime[j]=Phi_spp[j]-(Kx[j]*S[i][0]*DeltaX)-(Kspp_R[j]*L)-

Thi; $\quad / *$ plasmon phase delay at point $\mathrm{x} * /$ 
plasmon excitation and attenuation*/

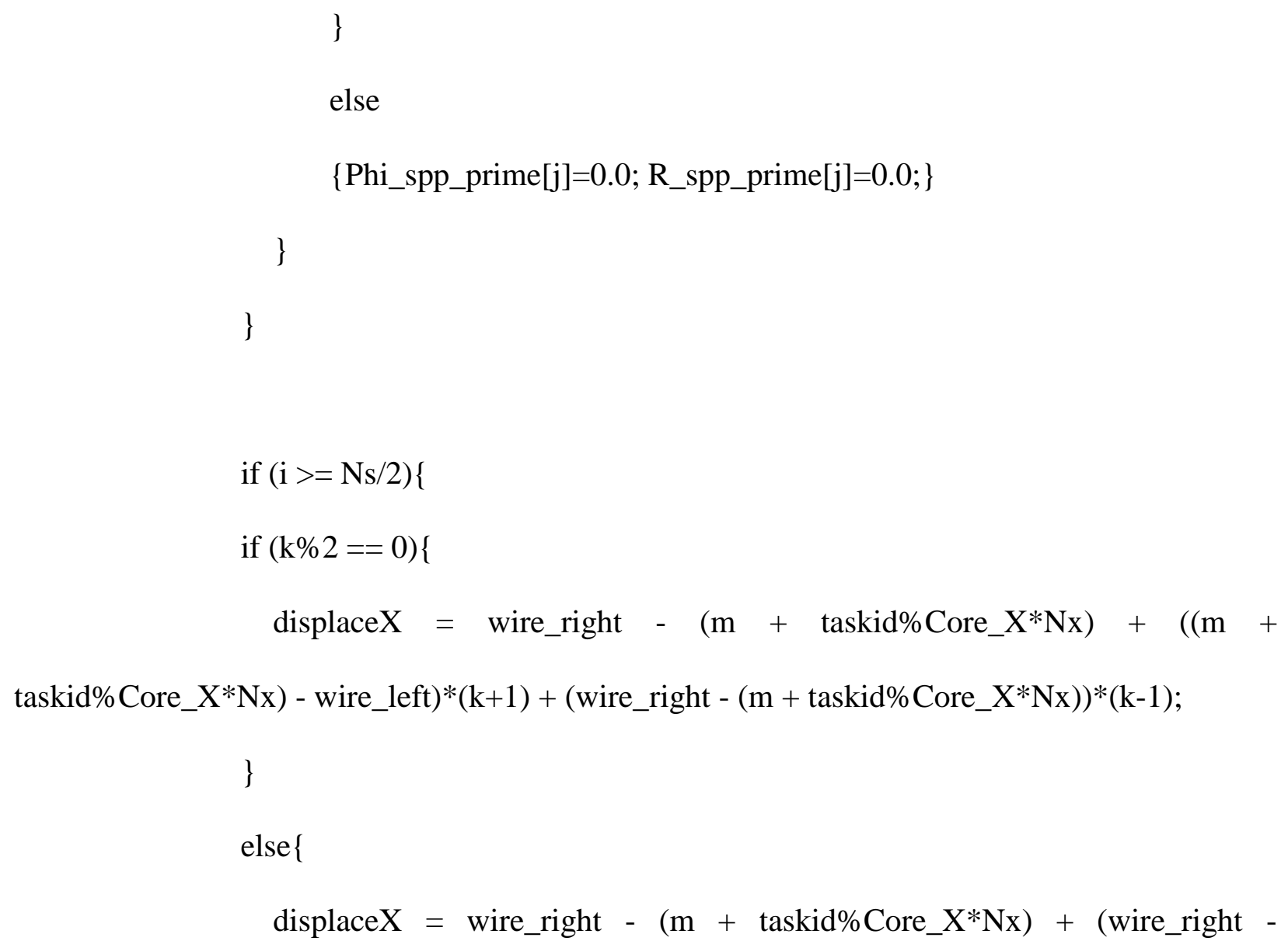

$$
\text { for }(\mathrm{j}=0 ; \mathrm{j}<\mathrm{N} ; \mathrm{j}++)\{
$$




$$
\operatorname{if}\left((\mathrm{j}>=1) \& \&\left(\mathrm{j}<=\left(\mathrm{f} \_ \text {center }+100\right)\right)\right)\{\quad \quad / * \text { only works around the }
$$

spectrum frequency where the dielectric constant is a smooth function*/

$$
\text { Phi_spp_prime[j] }=\text { Phi_spp[j]-(Kx[j]*S[i][0]*DeltaX)-(Kspp_R[j]*L)- }
$$

Thi; $\quad$ /*plasmon phase delay at point $\mathrm{x} * /$

$$
\text { R_spp_prime[j]=R_spp[j]*exp(-Kspp_I[j]*L); } \quad \text { /* }
$$

plasmon excitation and attenuation*/

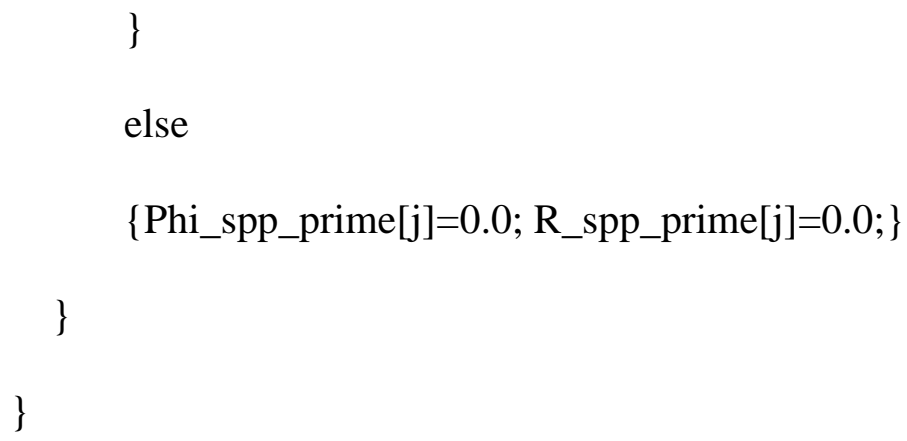

front half part

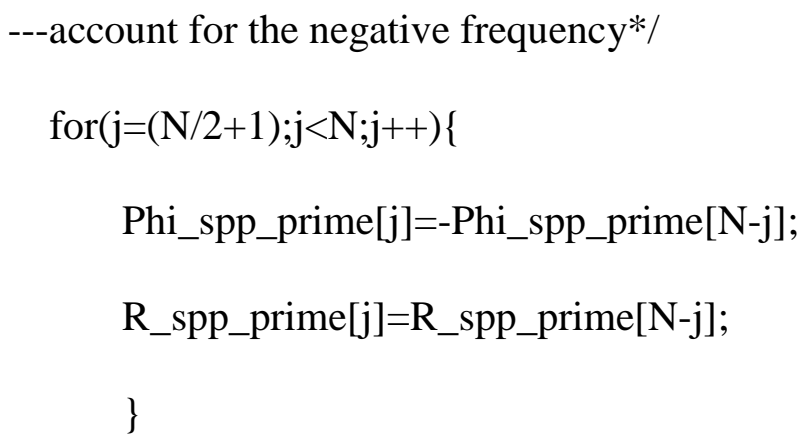

/*convert to cartesian coordinate*/

$$
\begin{aligned}
& \operatorname{for}(j=0 ; j<\mathrm{N} ; j++)\{ \\
& \text { F_spp[j][0]=R_spp_prime[j]* }{ }^{*} \cos (\text { Phi_spp_prime[j] }) \text {; } \\
& \text { F_spp[j][1]=R_spp_prime[j]* } \sin (\text { Phi_spp_prime[j] }) \text {; } \\
& \text { \} }
\end{aligned}
$$


$/ *$ do the inverse fft to recover the propagated spp wave packet*/ ifft(N,E_spp,F_spp);

for $(j=0 ; j<N ; j++)$

$\operatorname{Pspp}(m, n, j)+=\operatorname{pow}($ Ratio,k)*E_spp[j][0];

\}

\}

Ratio $=$ Ratio $*$ Ratio;

\}

\}

strcpy(file1,"P_SPPout");

sprintf(filename1,"\%s.\%d",file1,taskid);

$\mathrm{fp}=$ fopen(filename1,"w");

// $\quad$ fp=fopen("P_SPPout","w");

for $(\mathrm{m}=0 ; \mathrm{m}<\mathrm{Nx} ; \mathrm{m}++)\{$

for $(n=0 ; n<N y ; n++)\{$

for $(j=0 ; j<N ; j++)\{$

fprintf(fp,"\%f, ", Pspp(m,n,j));

\}

fprintf(fp,"\n");

\} 


$$
\text { scanf("\%d",\&choice); }
$$

$* /$

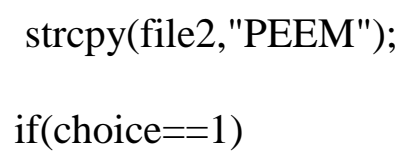




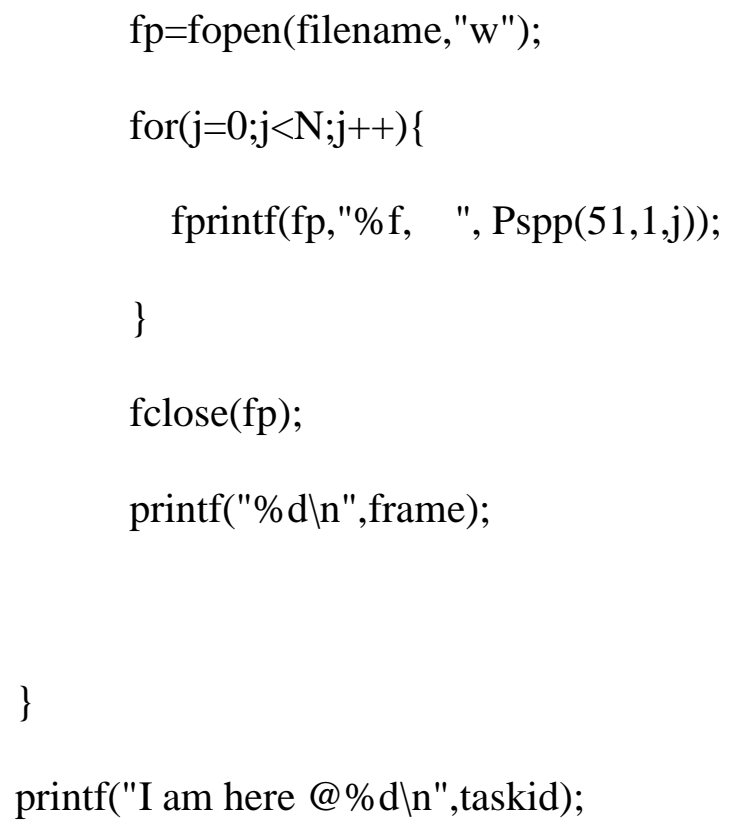


fwrite(\&dim1,sizeof(float),1,snapshot); fwrite(\&dim2,sizeof(float),1,snapshot);

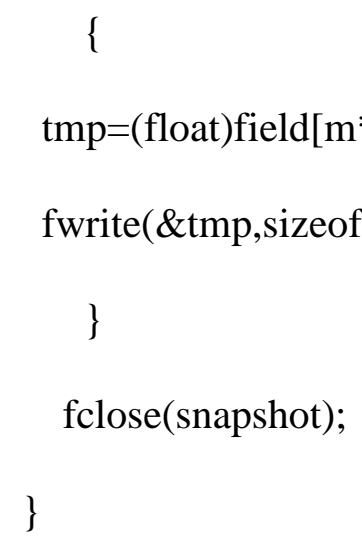




\section{Bibliography}

1. Johnson, P.B. and R.-W. Christy, Optical constants of the noble metals. Physical review B, 1972. 6(12): p. 4370.

2. Wang, Y., E. Plummer, and K. Kempa, Foundations of plasmonics. Advances in Physics, 2011. 60(5): p. 799-898.

3. Jackson, J.D., Classical electrodynamics. 2012: John Wiley \& Sons.

4. Maier, S.A., Plasmonics: fundamentals and applications. 2007: Springer Science \& Business Media.

5. Yang, H.U., et al., Optical dielectric function of silver. Physical Review B, 2015. 91(23): p. 235137.

6. Hartland, G.V., Optical studies of dynamics in noble metal nanostructures. Chemical reviews, 2011. 111(6): p. 3858-3887.

7. $\mathrm{Fu}, \mathrm{Q}$. and W. Sun, Mie theory for light scattering by a spherical particle in an absorbing medium. Applied Optics, 2001. 40(9): p. 1354-1361.

8. Liebsch, A., Surface-plasmon dispersion and size dependence of Mie resonance: silver versus simple metals. Physical Review B, 1993. 48(15): p. 11317.

9. Reiners, T., et al., Size dependence of the optical response of spherical sodium clusters. Physical review letters, 1995. 74(9): p. 1558.

10. Raether, H., Surface plasmons on gratings, in Surface plasmons on smooth and rough surfaces and on gratings. 1988, Springer. p. 91-116.

11. Bliokh, K.Y. and F. Nori, Transverse and longitudinal angular momenta of light. Physics Reports, 2015. 592: p. 1-38. 
12. Bliokh, K.Y., et al., Spin-orbit interactions of light. Nature Photonics, 2015. 9(12): p. 796.

13. Van Mechelen, T. and Z. Jacob, Universal spin-momentum locking of evanescent waves. Optica, 2016. 3(2): p. 118-126.

14. Bliokh, K.Y., A.Y. Bekshaev, and F. Nori, Extraordinary momentum and spin in evanescent waves. Nature communications, 2014. 5: p. 3300.

15. Bliokh, K.Y. and F. Nori, Transverse spin of a surface polariton. Physical Review A, 2012. 85(6): p. 061801.

16. Aiello, A., et al., From transverse angular momentum to photonic wheels. Nature Photonics, 2015. 9(12): p. 789.

17. Genevet, P., et al., Controlled steering of Cherenkov surface plasmon wakes with a onedimensional metamaterial. Nature Nanotechnology, 2015. 10(9): p. 804.

18. Kou, S.S., et al., On-chip photonic Fourier transform with surface plasmon polaritons. Light: Science \& Applications, 2016. 5(2): p. e16034.

19. Rodríguez-Fortuño, F.J., et al., Near-field interference for the unidirectional excitation of electromagnetic guided modes. Science, 2013. 340(6130): p. 328-330.

20. O'connor, D., et al., Spin-orbit coupling in surface plasmon scattering by nanostructures. Nature communications, 2014. 5: p. 5327.

21. Lin, J., et al., Polarization-controlled tunable directional coupling of surface plasmon polaritons. Science, 2013. 340(6130): p. 331-334.

22. Chen, C.-F., et al., Creating optical near-field orbital angular momentum in a gold metasurface. Nano letters, 2015. 15(4): p. 2746-2750.

23. Spektor, G., et al., Metafocusing by a metaspiral plasmonic lens. Nano letters, 2015. 15(9): p. $5739-5743$. 
24. Yu, N., et al., Light propagation with phase discontinuities: generalized laws of reflection and refraction. science, 2011: p. 1210713.

25. Yu, N. and F. Capasso, Flat optics with designer metasurfaces. Nature materials, 2014. 13(2): p. 139.

26. Gorodetski, Y., et al., Tracking surface plasmon pulses using ultrafast leakage imaging. Optica, 2016. 3(1): p. 48-53.

27. Gorodetski, Y., et al., Weak measurements of light chirality with a plasmonic slit. Physical review letters, 2012. 109(1): p. 013901.

28. Zhang, S., et al., Chiral surface plasmon polaritons on metallic nanowires. Physical review letters, 2011. 107(9): p. 096801.

29. Pan, D., et al., Strong spin-orbit interaction of light in plasmonic nanostructures and nanocircuits. Physical review letters, 2016. 117(16): p. 166803.

30. Dai, Y., et al., Ultrafast microscopy of spin-momentum-locked surface plasmon polaritons. ACS nano, 2018. 12(7): p. 6588-6596.

31. Dąbrowski, M., Y. Dai, and H. Petek, Ultrafast microscopy: Imaging light with photoelectrons on the nano-femto scale. The Journal of Physical Chemistry Letters, 2017. 8(18): p. 4446-4455.

32. Gong, Y., et al., Ultrafast imaging of surface plasmons propagating on a gold surface. Nano letters, 2015. 15(5): p. 3472-3478.

33. Gong, Y., et al., Nonlinear Photoemission Electron Micrographs of Plasmonic Nanoholes in Gold Thin Films. The Journal of Physical Chemistry C, 2014. 118(44): p. 25671-25676.

34. Gong, Y., et al., Polarization-Directed Surface Plasmon Polariton Launching. The Journal of Physical Chemistry Letters, 2016. 8(1): p. 49-54. 
35. Buckanie, N.M., et al., Interaction of light and surface plasmon polaritons in Ag islands studied by nonlinear photoemission microscopy. Ultramicroscopy, 2013. 130: p. 49-53.

36. Razinskas, G., et al., Normal-incidence PEEM imaging of propagating modes in a plasmonic nanocircuit. Nano letters, 2016. 16(11): p. 6832-6837.

37. Chelaru, L.I. and F.-J.M. zu Heringdorf, In situ monitoring of surface plasmons in singlecrystalline Ag-nanowires. Surface Science, 2007. 601(18): p. 4541-4545.

38. Chelaru, L., et al., Fringe fields in nonlinear photoemission microscopy. Physical Review B, 2006. 73(11): p. 115416.

39. zu Heringdorf, F.-J.M., et al., Femtosecond photoemission microscopy. Surface Science, 2007. 601(20): p. 4700-4705.

40. Lemke, C., et al., Spatiotemporal characterization of SPP pulse propagation in twodimensional plasmonic focusing devices. Nano letters, 2013. 13(3): p. 1053-1058.

41. Lemke, C., et al., Measurement of surface plasmon autocorrelation functions. Optics express, 2013. 21(22): p. 27392-27401.

42. Bauer, M., et al., Phase propagation of localized surface plasmons probed by time-resolved photoemission electron microscopy. Applied Physics A, 2007. 88(3): p. 473-480.

43. Telieps, W. and E. Bauer, An analytical reflection and emission UHV surface electron microscope. Ultramicroscopy, 1985. 17(1): p. 57-65.

44. Bauer, E., Low energy electron microscopy. Reports on Progress in Physics, 1994. 57(9): p. 895.

45. Bauer, E., A brief history of PEEM. Journal of Electron Spectroscopy and Related Phenomena, 2012. 185(10): p. 314-322. 
46. Tromp, R., et al., A new aberration-corrected, energy-filtered LEEM/PEEM instrument II. Operation and results. Ultramicroscopy, 2013. 127: p. 25-39.

47. Tromp, R., et al., A new aberration-corrected, energy-filtered LEEM/PEEM instrument. I. Principles and design. Ultramicroscopy, 2010. 110(7): p. 852-861.

48. Tromp, R., Measuring and correcting aberrations of a cathode objective lens. Ultramicroscopy, 2011. 111(4): p. 273-281.

49. Petek, H. and S. Ogawa, Femtosecond time-resolved two-photon photoemission studies of electron dynamics in metals. Progress in surface science, 1997. 56(4): p. 239-310.

50. Kubo, A., et al., Femtosecond imaging of surface plasmon dynamics in a nanostructured silver film. Nano letters, 2005. 5(6): p. 1123-1127.

51. Watanabe, K., et al., Photochemistry on metal nanoparticles. Chemical reviews, 2006. 106(10): p. 4301-4320.

52. Cavalieri, A.L., et al., Attosecond spectroscopy in condensed matter. Nature, 2007. 449(7165): p. 1029.

53. Onda, K., et al., Wet electrons at the H2O/TiO2 (110) surface. Science, 2005. 308(5725): p. $1154-1158$.

54. Onda, K., B. Li, and H. Petek, Two-photon photoemission spectroscopy of Ti O 2 (110) surfaces modified by defects and O 2 or $\mathrm{H} 2 \mathrm{O}$ adsorbates. Physical Review B, 2004. 70(4): p. 045415.

55. Krausz, F. and M. Ivanov, Attosecond physics. Reviews of Modern Physics, 2009. 81(1): p. 163.

56. Kubo, A., N. Pontius, and H. Petek, Femtosecond microscopy of surface plasmon polariton wave packet evolution at the silver/vacuum interface. Nano letters, 2007. 7(2): p. 470-475. 
57. Griffith, O.H. and W. Engel, Historical perspective and current trends in emission microscopy, mirror electron microscopy and low-energy electron microscopy: an introduction to the proceedings of the second international symposium and workshop on emission microscopy and related techniques. Ultramicroscopy, 1991. 36(1-3): p. 1-28.

58. Mundschau, M., Emission microscopy and surface science. Ultramicroscopy, 1991. 36(13): p. 29-51.

59. Schramm, S., et al., A Contrast Transfer Function approach for image calculations in standard and aberration-corrected LEEM and PEEM. Ultramicroscopy, 2012. 115: p. 88108.

60. Pang, A., et al., Fourier optics of image formation in LEEM. Journal of Physics: Condensed Matter, 2009. 21(31): p. 314006.

61. Baski, A., S. Erwin, and L. Whitman, The structure of silicon surfaces from (001) to (111). Surface Science, 1997. 392(1-3): p. 69-85.

62. Li, B., et al., A LEEM study of bamboo-like growth of Ag crystals on Si (0 O 1) surfaces. Surface science, 2004. 569(1-3): p. 142-148.

63. Winau, D., et al., Reconstructions and growth of Ag on Si $(001)(2 \times 1)$. Surface science, 1994. 303(1-2): p. 139-145.

64. Inaoka, T., et al., Two-dimensional plasmon in a metallic monolayer on a semiconductor surface: Exchange-correlation effects. Physical Review B, 2002. 66(24): p. 245320.

65. Logeeswaran, V., et al., Ultrasmooth silver thin films deposited with a germanium nucleation layer. Nano letters, 2008. 9(1): p. 178-182.

66. Tan, S., et al., Plasmonic coupling at a metal/semiconductor interface. Nature Photonics, 2017. 11(12): p. 806. 
67. Zhang, S., et al., Time-resolved photoemission study of the electronic structure and dynamics of chemisorbed alkali atoms on Ru (0001). Physical Review B, 2016. 93(4): p. 045401.

68. Taflove, A. and S.C. Hagness, Computational electrodynamics: the finite-difference timedomain method. 2005: Artech house.

69. Wang, L.M. and H. Petek, Focusing surface plasmon polariton wave packets in space and time. Laser \& Photonics Reviews, 2013. 7(6): p. 1003-1009.

70. Zhang, L., et al., Imaging of surface plasmon polariton fields excited at a nanometer-scale slit. Physical Review B Condensed Matter, 2011. 84(24): p. 2461-2468.

71. Wang, L.M. and H. Petek, Focusing surface plasmon polariton wave packets in space and time. Laser \& Photonics Reviews, 2013. 7(7): p. 1003-1009.

72. Genevet, P., et al. Controlled steering of Cherenkov surface plasmon wakes with a onedimensional metamaterial. in Lasers and Electro-Optics. 2015.

73. Dąbrowski, M., et al., Multiphoton Photoemission Microscopy of High-Order Plasmonic Resonances at the Ag/Vacuum and Ag/Si Interfaces of Epitaxial Silver Nanowires. ACS Photonics, 2016. 3(9): p. 1704-1713.

74. Born, M. and E. Wolf, Principles of optics: electromagnetic theory of propagation, interference and diffraction of light. 2013: Elsevier.

75. O'Connor, D., et al., Spin-orbit coupling in surface plasmon scattering by nanostructures. Nature Communications, 2014. 5: p. 5327.

76. Wang, Z., et al., Gyrotropic response in the absence of a bias field. Proceedings of the National Academy of Sciences, 2012. 109(33): p. 13194-13197. 
77. Leissner, T., et al., Surface plasmon polariton propagation in organic nanofiber based plasmonic waveguides. Optics Express, 2013. 21(7): p. 8251-60.

78. Buckanie, N.M., et al., Interaction of light and surface plasmon polaritons in Ag islands studied by nonlinear photoemission microscopy. Ultramicroscopy, 2013. 130(2): p. 49-53.

79. Kubo, A., N. Pontius, and H. Petek, Femtosecond Microscopy of Surface Plasmon Polariton Wave Packet Evolution at the Silver/Vacuum Interface. Nano Lett., 2007. 7(2): p. $470-475$.

80. Bliokh, K.Y., D. Smirnova, and F. Nori, Quantum spin Hall effect of light. Science, 2015. 348(6242): p. 1448-1451.

81. Yin, X., et al., Photonic spin Hall effect at metasurfaces. Science, 2013. 339(6126): p. 1405-1407.

82. Liu, Y., et al., Photonic spin Hall effect in metasurfaces: a brief review. Nanophotonics, 2017. 6(1): p. 51.

83. Dennis, M.R., K. O'Holleran, and M.J. Padgett, Singular optics: optical vortices and polarization singularities, in Progress in Optics. 2009, Elsevier. p. 293-363.

84. Tang, Y. and A.E. Cohen, Optical Chirality and Its Interaction with Matter. Physical Review Letters, 2010. 104(16): p. 163901.

85. Tang, Y. and A.E. Cohen, Enhanced enantioselectivity in excitation of chiral molecules by superchiral light. Science, 2011. 332(6027): p. 333-336.

86. Bliokh, K.Y. and F. Nori, Characterizing optical chirality. Physical Review A, 2011. 83(2): p. 021803.

87. Hendry, E., et al., Ultrasensitive detection and characterization of biomolecules using superchiral fields. Nature nanotechnology, 2010. 5(11): p. 783. 
88. Mak, K.F., et al., Control of valley polarization in monolayer MoS 2 by optical helicity. Nature nanotechnology, 2012. 7(8): p. 494.

89. Coullet, P., L. Gil, and F. Rocca, Optical vortices. Optics Communications, 1989. 73(5): p. 403-408.

90. Gahagan, K. and G. Swartzlander, Optical vortex trapping of particles. Optics Letters, 1996. 21(11): p. 827-829.

91. Kim, H., et al., Synthesis and dynamic switching of surface plasmon vortices with plasmonic vortex lens. Nano letters, 2010. 10(2): p. 529-536.

92. Tan, P., et al., Phase singularity of surface plasmon polaritons generated by optical vortices. Optics letters, 2011. 36(16): p. 3287-3289.

93. Tsai, W.-Y., J.-S. Huang, and C.-B. Huang, Selective trapping or rotation of isotropic dielectric microparticles by optical near field in a plasmonic archimedes spiral. Nano letters, 2014. 14(2): p. 547-552.

94. Spektor, G., et al., Revealing the subfemtosecond dynamics of orbital angular momentum in nanoplasmonic vortices. Science, 2017. 355(6330): p. 1187-1191.

95. Yang, S., et al., Miniature circular polarization analyzer with spiral plasmonic lens. Optics letters, 2009. 34(20): p. 3047-3049.

96. Davis, T.J., et al., Subfemtosecond and nanometer plasmon dynamics with photoelectron microscopy: theory and efficient simulations. ACS Photonics, 2017. 4(10): p. 2461-2469.

97. Li, J., et al., Plasmonic focusing in spiral nanostructures under linearly polarized illumination. Optics express, 2014. 22(14): p. 16686-16693.

98. Guo, Q., C. Zhang, and X. Hu, A spiral plasmonic lens with directional excitation of surface plasmons. Scientific reports, 2016. 6: p. 32345. 
99. Nagaosa, N. and Y. Tokura, Topological properties and dynamics of magnetic skyrmions. Nature nanotechnology, 2013. 8(12): p. 899.

100. Skyrme, T.H.R., A unified field theory of mesons and baryons. Nuclear Physics, 1962. 31: p. 556-569.

101. Al Khawaja, U. and H. Stoof, Skyrmions in a ferromagnetic Bose-Einstein condensate. Nature, 2001. 411(6840): p. 918.

102. Wright, D.C. and N.D. Mermin, Crystalline liquids: the blue phases. Reviews of Modern physics, 1989. 61(2): p. 385.

103. Abrikosov, A.A., Type II superconductors and the vortex lattice. Nobel Lecture, December, 2003. 8: p. 254.

104. Mühlbauer, S., et al., Skyrmion lattice in a chiral magnet. Science, 2009. 323(5916): p. 915-919.

105. Yu, X., et al., Real-space observation of a two-dimensional skyrmion crystal. Nature, 2010. 465(7300): p. 901.

106. Heinze, S., et al., Spontaneous atomic-scale magnetic skyrmion lattice in two dimensions. Nature Physics, 2011. 7(9): p. 713.

107. Kézsmárki, I., et al., Néel-type skyrmion lattice with confined orientation in the polar magnetic semiconductor GaV 4 S 8. Nature materials, 2015. 14(11): p. 1116.

108. Woo, S., et al., Observation of room-temperature magnetic skyrmions and their currentdriven dynamics in ultrathin metallic ferromagnets. Nature materials, 2016. 15(5): p. 501.

109. Yu, X., et al., Skyrmion flow near room temperature in an ultralow current density. Nature communications, 2012. 3: p. 988. 
110. Fösel, T., V. Peano, and F. Marquardt, L lines, $C$ points and Chern numbers: understanding band structure topology using polarization fields. New Journal of Physics, 2017. 19(11): p. 115013.

111. Horn, B.K. and B.G. Schunck, Determining optical flow. Artificial intelligence, 1981. 17(1-3): p. 185-203. 